\title{
Bases de Hilbert
}

\author{
Marcelo Hashimoto
}

DISSERTAÇÃO APRESENTADA

$\mathrm{AO}$

INSTITUTO DE MATEMÁTICA E ESTATÍSTICA

DA

UNIVERSIDADE DE SÃO PAULO

PARA

OBTENÇÃO DO TÍTULO DE MESTRE

EM

CIENCIAS

\begin{abstract}
Área de Concentração: Ciência da Computação
Orientador: Prof. Dr. José Coelho de Pina Junior
\end{abstract}

Durante a elaboração deste trabalho o autor recebeu auxílio financeiro da CAPES

São Paulo, outubro de 2007. 



\section{Bases de Hilbert}

Este exemplar corresponde à redação final da dissertação devidamente corrigida e defendida por Marcelo Hashimoto e aprovada pela comissão julgadora.

Banca examinadora:

Prof. Dr. José Coelho de Pina Junior (presidente) IME-USP

Prof. Dr. Paulo Feofiloff IME-USP

Prof. Dr. Orlando Lee IC-UNICAMP 



\section{Agradecimentos}

Não tenho a pretensão necessária para acreditar que conseguirei me lembrar de todas as pessoas a quem devo agradecer pela existência desta dissertação. Durante seis longos anos de graduação e mestrado, foram muitas as pessoas que direta ou indiretamente contribuíram para o término deste trabalho. Assim, para aqueles que foram esquecidos, deixo registradas sinceras desculpas por não os ter mencionado.

Primeiramente, gostaria de agradecer ao Coelho pela excelente orientação. Em particular, sou grato por todas as ocasiões nas quais ele me apresentou pontos de vista mais intuitivos para o que estudávamos: aprendi o valor de compreender um mesmo resultado a partir de várias perspectivas distintas e a importância de ter uma mente aberta.

Agradeço muito aos professores Orlando Lee, Paulo Feofiloff e José Augusto pela paciência, pelas correções e pelos valiosos conselhos apresentados durante minha qualificação e minha defesa. Ao Paulo devo agradecer duas vezes, pois ele participou dos dois eventos.

Agradeço também à Cris, à Yoshiko, ao Yoshi, ao Carlinhos e ao Arnaldo, que tanto dentro como fora das salas de aula tiveram um papel fundamental em minha formação.

Meus colegas foram responsáveis por tornar o ambiente acadêmico muito mais agradável, então em retribuição me esforçarei ao máximo para lembrar de todos. Agradecimentos ao Marcel, Cardonha, Ju, Wendel, Luna, Goca, Fernando Mário, Morgato, Fabrécio, Maya, Fred, Cristiane, Márcio e os Domingos Dellamonica e Soares pelas discussões interessantes durante meus anos de combinatória. Mais agradecimentos a eles e também ao Salem, Felipe,

Ricardo, Facci, Wanderley, Chicão, Lobato, Antonio Carlos e Marina pelas agradáveis tardes de procrastinação no laboratório. Outros agradecimentos ao Otávio, ao Leo, ao Nelson e a todos os outros Imescos ${ }^{\mathrm{TM}}$ pelos divertidos eventos.

Reservei os últimos agradecimentos para pessoas muito especiais, às quais dedico esta dissertação: meus irmãos, meus pais, que sempre apoiaram minhas decisões, e minha querida Ellen, cujo afeto me traz felicidade há anos e me sustenta durante os momentos mais difíceis. 



\title{
Resumo
}

Muitas relações min-max em otimização combinatória podem ser demonstradas através de total dual integralidade de sistemas lineares. O conceito algébrico de bases de Hilbert foi originalmente introduzido com o objetivo de melhor compreender a estrutura geral dos sistemas totalmente dual integrais.

Resultados apresentados posteriormente mostraram que bases de Hilbert também são relevantes para a otimização combinatória em geral e para a caracterização de certas classes de objetos discretos. Entre tais resultados, foram provadas, a partir dessas bases, versões do teorema de Carathéodory para programação inteira.

Nesta dissertação, estudamos aspectos estruturais e computacionais de bases de Hilbert e relações destas com programação inteira e otimização combinatória. Em particular, consideramos versões inteiras do teorema de Carathéodory e conjecturas relacionadas.

\begin{abstract}
There are several min-max relations in combinatorial optimization that can be proved through total dual integrality of linear systems. The algebraic concept of Hilbert basis was originally introduced with the objective of better understanding the general structure of totally dual integral systems.

Some results that were proved later have shown that Hilbert basis are also relevant to combinatorial optimization in a general manner and to characterize certain classes of discrete objects. Among such results, there are versions of Carathéodory's theorem for integer programming that were proved through those basis.

In this dissertation, we study structural and computational aspects of Hilbert basis and their relations to integer programming and combinatorial optimization. In particular, we consider integer versions of Carathéodory's theorem and related conjectures.
\end{abstract}





\section{Sumário}

$\begin{array}{ll}\text { Introdução } & 1\end{array}$

$\begin{array}{lr}\text { Preliminares } & 7\end{array}$

1 Sistemas e fechos lineares $\quad 11$

1.1 Método da eliminação gaussiana . . . . . . . . . . . . . . . . . 11

1.2 Correção da eliminação gaussiana . . . . . . . . . . . . . . . . . . 15

1.3 Eficiência da eliminação gaussiana . . . . . . . . . . . . . . . . 17

1.4 Fechos lineares e bases . . . . . . . . . . . . . . . . . . . . 21

2 Desigualdades lineares e cones $\quad 25$

2.1 Teorema das desigualdades lineares . . . . . . . . . . . . . . . . 25

2.2 Programação linear e dualidade . . . . . . . . . . . . . . . . . . . . 27

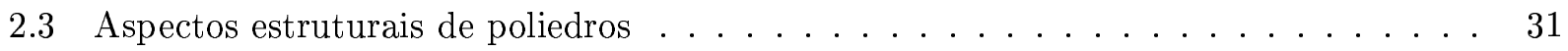

2.4 Cones e o teorema de Carathéodory . . . . . . . . . . . . . . . 33

3 Sistemas diofantinos e reticulados $\quad 37$

3.1 Algoritmo de Euclides . . . . . . . . . . . . . . . . . . . . . . 37

3.2 Equações lineares diofantinas . . . . . . . . . . . . . . . . . . . . 39

3.3 Sistemas lineares diofantinos . . . . . . . . . . . . . . . . . . . 41

3.4 Forma normal de Hermite . . . . . . . . . . . . . . . . . . . . . . . . 44

3.5 Matrizes unimodulares . . . . . . . . . . . . . . . . . . . 47

3.6 Reticulados e bases . . . . . . . . . . . . . . . . . 50

4 Cones inteiros e bases de Hilbert

4.1 Cones inteiros . . . . . . . . . . . . . . . . . . . . 53

4.2 Sistemas geradores de Hilbert . . . . . . . . . . . . . . . . . . 55

4.3 Bases de Hilbert . . . . . . . . . . . . . . . . . . . . . . . . . . . . . . . . . . . . . . . . . . . . . . .

4.4 Algoritmo de Chandrasekaran . . . . . . . . . . . . . . . . . . . . . . . . . . . . . . . . .

4.5 Bases isomorfas e imersões em reticulados . . . . . . . . . . . . . . . . . 60

4.6 Bases totalmente integrais . . . . . . . . . . . . . . . . . . . 61

5 Programação inteira e total dual integralidade $\quad 63$

5.1 Programação inteira e poliedros inteiros . . . . . . . . . . . . . . . 63

5.2 Sistemas totalmente dual integrais . . . . . . . . . . . . . . . . 65

5.3 Algoritmos em dimensão fixa . . . . . . . . . . . . . . . . . . 67 
6 Versões inteiras do teorema de Carathéodory $\quad 71$

6.1 Teorema de Cook, Fonlupt e Schrijver . . . . . . . . . . . . . . . . . 71

6.2 Conjecturas de Sebő . . . . . . . . . . . . . . . . . . . 76

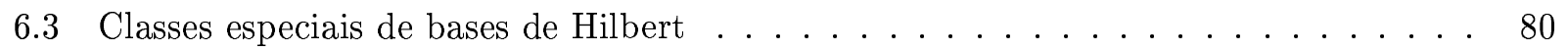

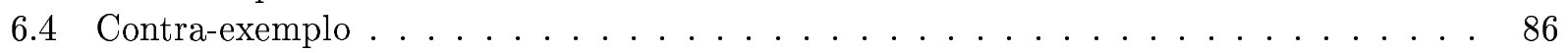

7 Bases de Hilbert e combinatória $\quad \mathbf{8 9}$

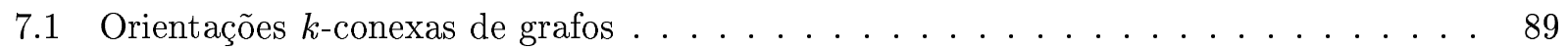

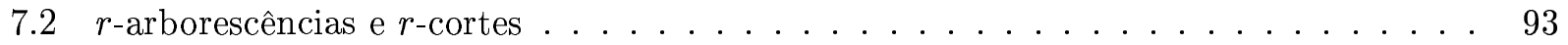

7.3 Cliques de grafos perfeitos . . . . . . . . . . . . . . . . . 96

7.4 Conjuntos gerados por circuitos . . . . . . . . . . . . . . . . . . . . 99

7.5 Florestas maximais e árvores geradoras . . . . . . . . . . . . . . . . . 116

$\begin{array}{ll}\text { Conclusão } & 131\end{array}$

$\begin{array}{ll}\text { Bibliografia } & 133\end{array}$ 


\section{Introdução}

Imaginemos uma criança. Crianças nada mais são do que indivíduos que estão vivos há pouco tempo. Para compreender o significado de pouco nessa definição, o leitor deve se desvencilhar da frieza dos números e pensar principalmente em insuficiência; em pessoas cuja ingenuidade ainda não foi suficientemente maculada pela experiência; em criaturas cujo entusiasmo ainda pode ser despertado pelo mundo; em seres para quem a existência ainda não se transformou em uma amarga trivialidade; em infantilidade espiritual, não meramente física.

Contemplemos essa criança diante de um doce e de outra criança. O doce é o seu objeto de desejo. A outra criança é o seu rival. Quando o desejo e a rivalidade ocorrem simultaneamente, ambos tornam-se as sementes de um conflito que pode irremediavelmente destruir relacionamentos ou estilhaçar sonhos. Uma criança normalmente possui a obstinação necessária para enfrentar tal conflito, mas raramente possui a sabedoria necessária para conseguir resolvê-lo.

Imaginemos agora um adulto. Estamos nos referindo a um indivíduo que está vivo há muito tempo. Tempo suficiente para transformar a ingenuidade em cinismo, suficiente para converter o entusiasmo em tédio, para remover da existência tudo o que há de especial. Contemplemos esse adulto diante de duas mulheres, incapaz de optar por uma delas. Ambas apresentam virtudes equivalentemente intensas e defeitos igualmente irrelevantes. Um adulto que sustenta experiências de peso considerável sobre seus ombros normalmente exibe uma racionalidade capaz de comparar duas mulheres sob o ponto de vista físico e material, mas raramente exibe os sentimentos capazes de considerar uma delas especial, independentemente da sua aparência ou dos seus bens.

A criança não compreende que poderia simplesmente compartilhar o doce com a outra. $\mathrm{O}$ adulto não compreende que deveria valorizar a importância do aspecto emocional. Uma criança tem admirável paixão, mas sofre por não ter conhecimento. Um adulto tem respeitável conhecimento, mas sofre por não ter paixão. Tanto a paixão como o conhecimento não são capazes de resolver todos os problemas sozinhos. Emoções podem criar laços permanentes entre amantes, mas podem dissolver eternamente laços entre rivais. Duas crianças podem compartilhar um doce, mas duas mulheres não podem compartilhar a alma de um homem.

O poeta francês Charles Baudelaire certa vez afirmou que há muito em comum entre aquilo que nós chamamos de inspiração e a alegria espontânea com a qual uma criança consegue absorver as cores e as formas do mundo. Ressaltou, no entanto, que todo o material abstrato acumulado por essa absorção só pode ser adequadamente organizado pelo pensamento racional de um adulto. A genialidade, portanto, nada mais seria do que um redescobrimento da infância. Uma capacidade intrínseca de inebriar-se com o mundo e ao mesmo tempo saber expressá-lo.

O contexto no qual as afirmações de Baudelaire estão localizadas é a arte. Mais especificamente, o poeta refere-se à inspiração e à genialidade de pintores. A área onde esta dissertação de mestrado se encontra é a ciência da computação. O conteúdo deste texto é o resultado do esforço de matemáticos. A pertinência da matemática ao escopo das artes é uma questão filosófica deveras complicada, na 
qual não pretendemos nos aprofundar. No entanto, ela não precisa ser esclarecida para que possamos adaptar as afirmações de Baudelaire a este trabalho.

Neste mestrado, estudamos conjuntos gerados por combinações inteiras não-negativas de vetores. Apesar de sua razoável extensão, a expressão combinação inteira não-negativa nada mais é do que um sinônimo excessivamente sofisticado de soma. Uma combinação inteira não envolve frações. Uma combinação não-negativa não envolve subtrações. Estudar combinações que são ao mesmo tempo inteiras e não-negativas significa, sob certos aspectos, revisitar a época em que não conhecíamos nenhum conceito matemático além da soma. Redescobrir a época em que éramos simples crianças, incapazes de aplicar o conceito de divisão sobre um doce. No entanto, ao rever um cenário que esteve presente em nossa infância, não é necessário que o contemplemos apenas com olhos juvenis. Podemos combinar a curiosidade infantil com a maturidade matemática.

Um equilíbrio adequado entre esses dois elementos é necessário para o estudo de problemas de otimização combinatória. Nesses problemas, o objetivo é otimizar uma função em um conjunto discreto e normalmente finito. Uma primeira impressão a respeito dessa descrição é a de que ela descreve problemas simples. Mais simples do que uma otimização em conjuntos contínuos, pois de certa forma estes são maiores e mais complexos. Trata-se, no entanto, de uma impressão equivocada. Conjuntos discretos não são simplesmente menores, eles são mais restritos. Conjuntos contínuos não são simplesmente complexos, eles são mais flexíveis.

Os problemas combinatórios são formulados dentro de um mundo discreto, no qual os doces são tão indivisíveis quanto para uma criança. No entanto, aqueles que estudam otimização combinatória não são crianças presas a técnicas discretas. São adultos capazes de utilizar técnicas variadas, sendo muitas delas baseadas em conceitos contínuos. São pesquisadores entusiasmados e ao mesmo tempo estudiosos, que podem reconhecer em sua área uma infância redescoberta na ciência da computação.

Em 1888, o matemático David Hilbert demonstrou um conhecido teorema a respeito da existência de bases finitas para ideais. Quase um século depois, em 1979, uma versão desse teorema para o conjunto dos vetores inteiros em um dado cone foi considerada por Giles e Pulleyblank.

Ao estudarem bases para vetores inteiros de cones, que merecidamente ficaram conhecidas como bases de Hilbert, os dois autores tinham como objetivo aprofundar-se no estudo do conceito conhecido como total dual integralidade, que havia sido introduzido por Edmonds e Giles alguns anos antes. O trabalho que apresentou sistemas totalmente dual integrais buscava unificar e estender certos resultados clássicos em combinatória, como o teorema de Edmonds para intersecção de matróides e o teorema de Lucchesi e Younger para empacotamento de cortes dirigidos.

Muitas relações min-max em otimização combinatória podem ser demonstradas através de total dual integralidade. Tais relações são particularmente importantes porque são estabelecidas a partir de uma versão inteira do conhecido teorema da dualidade de programação linear. Sempre que um problema pode ser formulado como um programa linear, o teorema da dualidade lhe dá uma nova perspectiva. No caso de problemas combinatórios, uma versão inteira da dualidade garante que essa nova perspectiva também admite formulação combinatória.

Caracterizações demonstradas por Giles e Pulleyblank e por Schrijver mostraram que bases de Hilbert são, de certa forma, abstrações de sistemas totalmente dual integrais. Quase todos os teoremas a respeito dessas bases, têm alguma conseqüência direta em total dual integralidade. No entanto, não é necessário que o estudo dessas bases tenha tais conseqüências como objetivo. De fato, a importância delas pode ser estendida para muito além dos sistemas totalmente dual integrais. 
Cook, Fonlupt e Schrijver demonstraram uma versão inteira do teorema de Carathéodory para bases de Hilbert e conseqüências diretas dessa versão sobre cliques de grafos perfeitos. Essa versão inteira foi refinada em um artigo de Sebô, que também apresentou algumas conjecturas a respeito de refinamentos ainda melhores e mostrou uma interpretação de diversos problemas em otimização combinatória sob o ponto de vista dessas conjecturas.

Com o auxílio de um computador, Bruns, Gubeladze, Henk, Martin e Weismantel exibiram um contra-exemplo para todas as conjecturas enunciadas. Entretanto, o próprio Sebó apresentou demonstrações não-triviais para diversos casos mais restritos delas e não se sabe se as conjecturas valem para vários dos objetos combinatórios para os quais elas fazem sentido.

Este mestrado tem como objetivo apresentar um tratamento uniforme e esmiuçado dos aspectos estruturais e computacionais de bases de Hilbert, além das relações dessas bases com sistemas totalmente dual integrais, versões inteiras do teorema de Carathéodory e problemas combinatórios.

No capítulo 1 apresentamos fechos lineares, os conjuntos de combinações lineares arbitrárias. São os conjuntos menos restritos sobre os quais iremos trabalhar e, precisamente por esse motivo, são aqueles sobre os quais podemos demonstrar diversos resultados fortes. Pode-se dizer que o objetivo dos capítulos posteriores é obter resultados análogos aos deste capítulo sob certas restrições.

No capítulo 2 apresentamos cones, os conjuntos de combinações lineares não-negativas. Existem relações profundas entre cones e programas lineares e este capítulo estabelece essas relações através de diversos resultados clássicos em programação linear. Em particular, um desses resultados é o teorema de Carathéodory, que passa a ter uma importância especial a partir do capítulo 6 .

No capítulo 3 apresentamos reticulados, os conjuntos de combinações lineares inteiras. Um dos aspectos mais interessantes dos resultados apresentados nesse capítulo é o fato de que muitos problemas são resolvidos a partir de extensões de um dos algoritmos mais clássicos da teoria dos números: o algoritmo de Euclides para determinar o máximo divisor comum de inteiros.

No capítulo 4 apresentamos cones inteiros, os conjuntos de combinações lineares que são ao mesmo tempo inteiras e não-negativas. Esse capítulo estabelece logo no início que trabalhar com esses conjuntos é difícil no caso geral. O conceito de bases de Hilbert é então introduzido como uma alternativa mais tratável, porém ainda suficientemente flexível para ser interessante.

No capítulo 5 descrevemos o papel do conceito de total dual integralidade em programação inteira e demonstramos os resultados que estabelecem a relação entre bases de Hilbert e sistemas totalmente dual integrais. Esses resultados são particularmente importantes porque representam a motivação original de Giles e Pulleyblank para a criação do conceito de bases de Hilbert.

No capítulo 6 consideramos versões inteiras do teorema de Carathéodory que foi introduzido no capítulo 2. O ponto de partida desse capítulo é o importante resultado de Cook, Fonlupt e Schrijver e as considerações feitas por Sebő a respeito desse resultado. São apresentadas então as conjecturas de Sebô e demonstrações dessas conjecturas em vários casos particulares.

No capítulo 7 exibimos alguns exemplos combinatórios relacionados a bases de Hilbert. Uma parte dos exemplos é baseada em total dual integralidade e relações min-max. A outra parte em conjuntos gerados por vetores de incidência, sem envolver otimização. Versões inteiras do teorema de Carathéodory também são relacionadas a alguns desses exemplos.

Nas duas páginas a seguir, apresentamos duas versões de um fluxograma que ilustra os tópicos desta dissertação. Na primeira versão, o ponto de vista é o de sistemas lineares. Na segunda versão, o ponto de vista é o de conjuntos gerados. As flechas apontam sempre para uma versão mais restrita. 


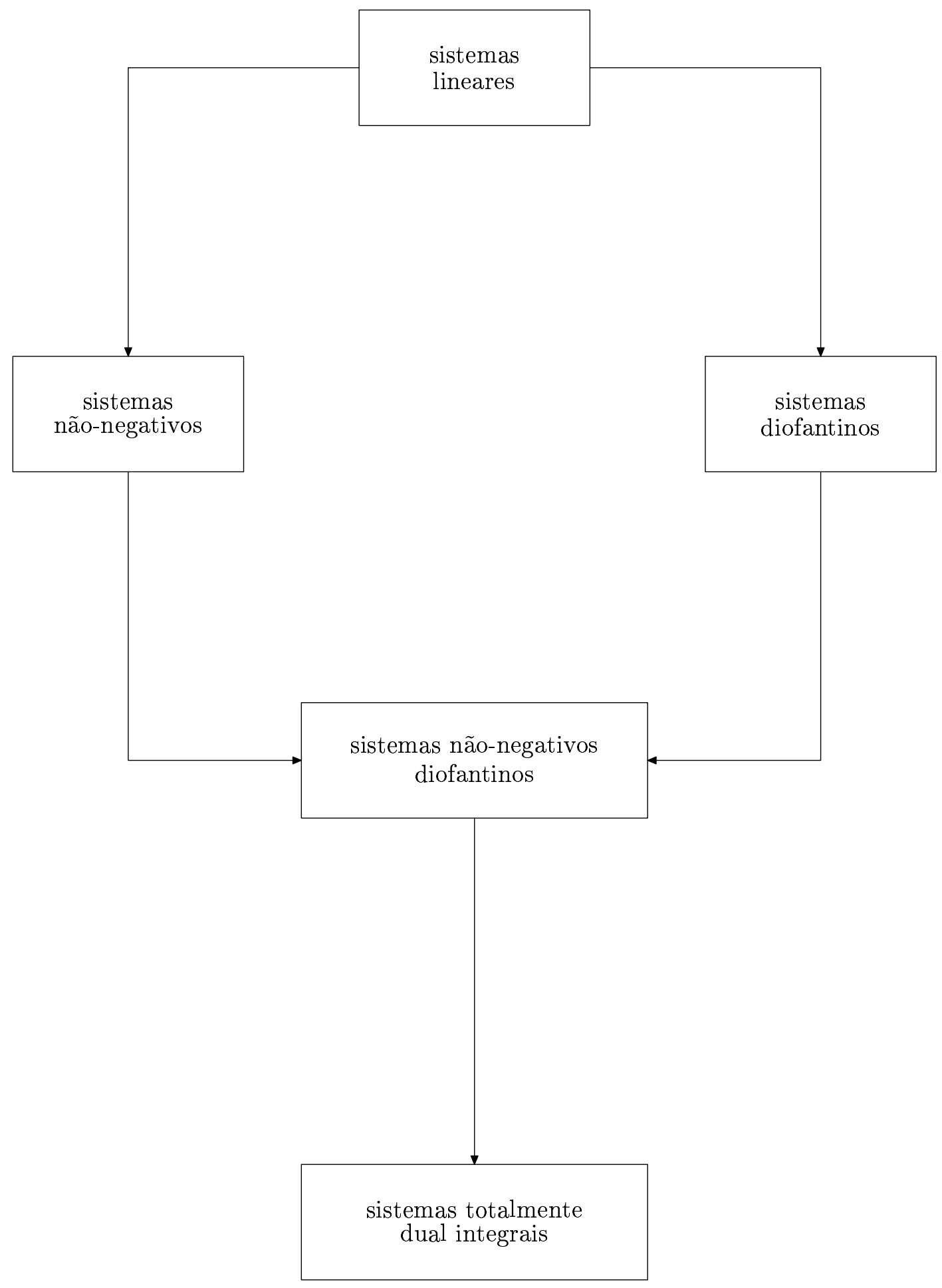




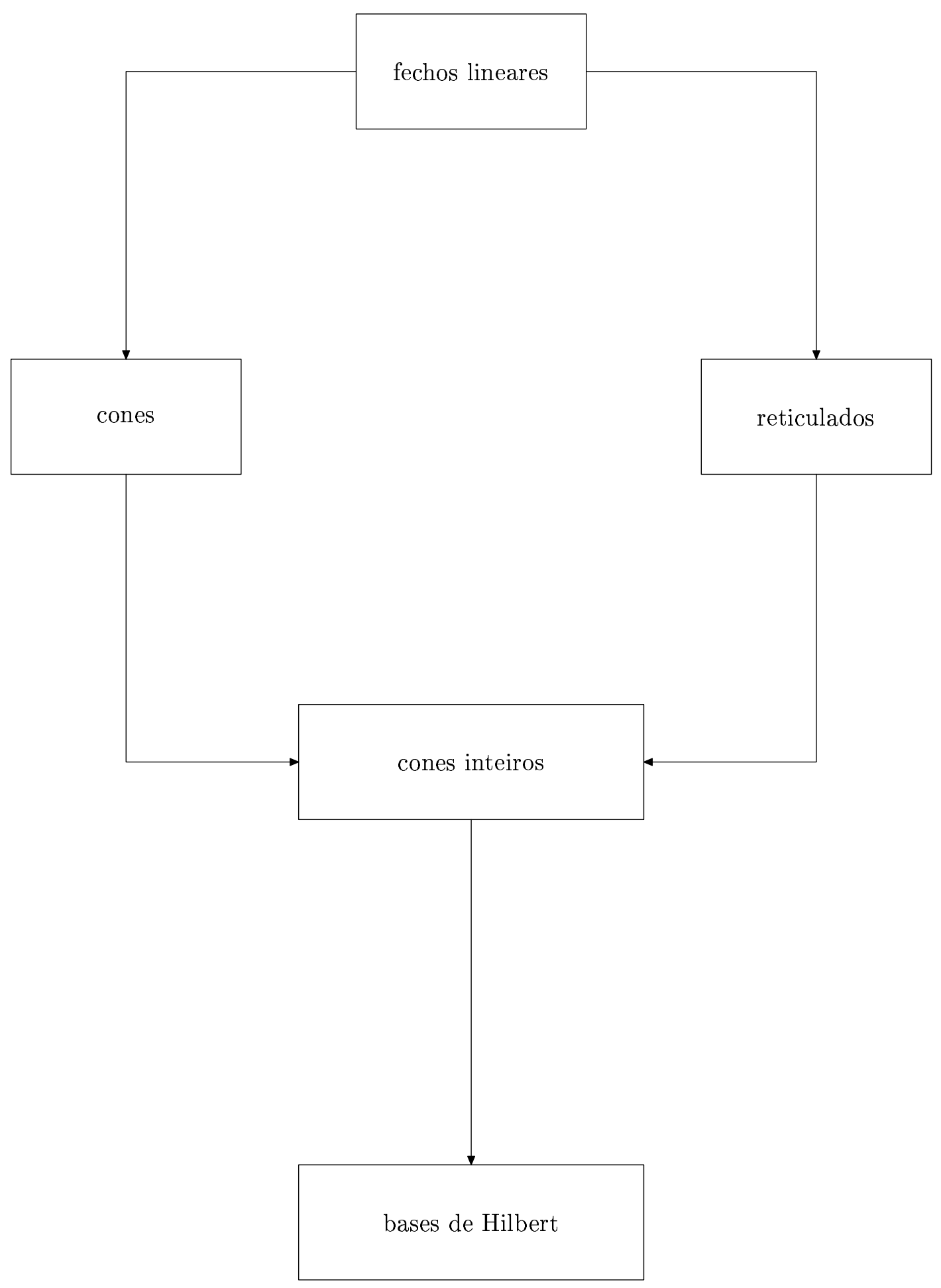





\section{Preliminares}

Na elaboração deste texto, buscamos uma abordagem razoavelmente auto-contida, mas estamos supondo que o leitor já teve algum contato com a terminologia e teoremas básicos de álgebra linear, complexidade computacional e teoria dos grafos. Listamos aqui alguns dos conceitos e resultados que serão utilizados, mas sem demonstrações ou definições formais.

Ao leitor que esteja interessado em ver detalhadamente esses conceitos, recomendamos o livro de PAPADimitriou [1994] sobre complexidade computacional e o livro de Diestel [2005] sobre teoria dos grafos. Já os conceitos de álgebra linear podem ser encontrados em qualquer livro introdutório.

\section{Álgebra linear}

Freqüentemente, consideraremos os conceitos de independência linear, posto e dimensão. Quando mencionarmos a dimensão de um conjunto de vetores, estaremos nos referindo à cardinalidade de um subconjunto linearmente independente maximal desse conjunto. O termo não irá se referir ao espaço no qual eles estão contidos. Por exemplo, diremos que a dimensão de um conjunto de dois vetores linearmente independentes em $\mathbb{R}^{n}$ é 2 , independentemente do valor de $n$.

Os conceitos de dimensão e posto, portanto, serão quase equivalentes. A principal diferença estará no fato de que o primeiro termo será sempre utilizado para conjuntos de vetores e o segundo sempre será utilizado para matrizes. Vamos dizer que uma matriz tem posto linha completo se seu posto é igual a seu número de linhas e que uma matriz tem posto coluna completo se seu posto é igual a seu número de colunas.

Alguns tipos básicos de matrizes, como matrizes diagonais e triangulares, serão fundamentais nos primeiros capítulos. Também utilizaremos algumas fórmulas básicas, como a fórmula de Laplace para determinantes e a fórmula para cálculo de matrizes inversas baseada na regra de Cramer.

As possíveis operações elementares sobre linhas de uma matriz são:

(i) permutação de linhas;

(ii) multiplicação de uma linha por um escalar;

(iii) soma de uma linha multiplicada por um escalar a outra.

As possíveis operações elementares sobre colunas de uma matriz são:

(i) permutação de colunas;

(ii) multiplicação de uma coluna por um escalar;

(iii) soma de uma coluna multiplicada por um escalar a outra. 
Para qualquer uma das operações sobre linhas, existe uma matriz não-singular $L$ tal que $L A$ é a matriz que obtemos após aplicar a operação sobre a matriz $A$. Para qualquer uma das operações sobre colunas, existe uma matriz não-singular $Q$ tal que $A Q$ é a matriz que obtemos após aplicar a operação sobre a matriz $A$. Tanto para operações sobre linhas como para operações sobre colunas, o determinante dessas matrizes pelas quais multiplicamos $A$ é igual a -1 quando são do tipo (i), é igual a 1 quando são do tipo (iii) e é igual ao escalar da multiplicação quando são do tipo (ii).

Por fim, o fecho convexo de um conjunto de vetores $a_{1}, \ldots, a_{m}$ é dado por:

$$
\operatorname{conv}\left(a_{1}, \ldots, a_{m}\right):=\left\{a: a=\lambda_{1} a_{1}+\cdots+\lambda_{m} a_{m} ; \lambda_{1}, \ldots, \lambda_{m} \geq 0 ; \lambda_{1}+\cdots+\lambda_{m}=1\right\} .
$$

Em demonstrações do capítulo 2, propriedades de fechos e combinações convexas serão utilizadas.

\section{Complexidade computacional}

Os conceitos de algoritmo polinomial e equivalência polinomial entre problemas está presente em praticamente todas as partes desse trabalho que estão relacionadas a algoritmos. Esses conceitos são conhecidos, mas faremos algumas observações a respeito de tamanho dos dados.

Os algoritmos que serão apresentados neste trabalho sempre consideram números racionais. Vamos sempre supor que um número é representado por dois inteiros de precisão arbitrária ao invés da abordagem usual de ponto flutuante. Embora essa representação evite problemas de precisão, ela faz com que o tamanho dos números não seja limitado superiormente.

Para demonstrar que os algoritmos apresentados são polinomiais, portanto, precisamos mostrar que o número de iterações e o tamanho dos números considerados ao longo da execução do algoritmo são polinomialmente limitados pelo tamanho da entrada.

Seja $A \in \mathbb{Q}^{n \times m}$ uma matriz, seja $a \in \mathbb{Q}^{n}$ um vetor e seja $\alpha \in \mathbb{Q}$ um escalar. Vamos denotar por $\alpha_{i, j}$ o elemento na linha $i$ e na coluna $j$ de $A$ e vamos denotar por $\alpha_{i}$ o $i$-ésimo elemento de $a$. Vamos também supor que $\alpha$ sempre é representado pelos menores inteiros possíveis $\mu, \delta$ tais que $\alpha=\mu / \delta$ e $\delta>0$. A definição de tamanho que utilizaremos é dada por pelas seguintes definições:

$$
\begin{aligned}
\operatorname{tam}(\alpha) & =\lceil\log (|\mu|+1)\rceil+\lceil\log (\delta+1)\rceil+1 ; \\
\operatorname{tam}(A) & =\sum_{i=1}^{n} \sum_{j=1}^{m} \operatorname{tam}\left(\alpha_{i, j}\right)+n+m ; \\
\operatorname{tam}(a) & =\sum_{i=1}^{n} \operatorname{tam}\left(\alpha_{i}\right)+n,
\end{aligned}
$$

onde a base do logaritmo é 2 . Em cálculos de complexidade, sempre consideraremos essas definições.

\section{Teoria dos grafos}

Conceitos de teoria dos grafos estão presentes apenas no capítulo 7, mas algumas das definições mais básicas serão esclarecidas aqui para que não precisemos desviar desnecessariamente o foco do leitor quando precisarmos delas posteriormente.

O padrão utilizado será o de que grafos admitem arestas paralelas mas não admitem laços. Da mesma forma, grafos dirigidos admitem arcos paralelos mas não admitem laços. Diremos que um grafo é simples se não admite arestas paralelas. Um grafo é definido como um par $(V, E)$, onde $V$ é o conjunto de vértices e $E$ é o conjunto de arestas. Analogamente, um grafo dirigido é definido como um par $(N, A)$, onde $N$ é o conjunto de vértices e $A$ é o conjunto de arcos.

Circuitos, florestas e árvores geradoras serão considerados conjuntos de arestas. Da mesma maneira, suas versões dirigidas serão consideradas conjuntos de arcos. O corte de um subconjunto 
de vértices $V^{\prime}$ de um grafo será denotado por $\delta\left(V^{\prime}\right)$. Em particular, em um grafo dirigido, $\underline{\delta}_{\rightarrow}\left(N^{\prime}\right)$ denota o conjunto dos arcos que entram em $N^{\prime}$ e $\_\left(N^{\prime}\right)$ denota o conjunto dos arcos que saem de $N^{\prime}$. Se $\mathcal{P}$ é uma partição do conjunto de vértices de um grafo simples, $\delta(\mathcal{P})$ denota o conjunto de arestas cujas pontas estão em partes distintas dessa partição.

Um caminho cujas pontas são os vértices $u, v$ será chamado de $(u, v)$-caminho. Para estender essa notação a grafos dirigidos, o primeiro vértice é a ponta inicial e o segundo vértice a ponta final. Alguns resultados clássicos em teoria dos grafos serão usados posteriormente como "caixa-preta".

- diferença simétrica de circuitos: se $C^{\prime}$ e $C^{\prime \prime}$ são circuitos de um grafo, então a diferença simétrica $\left(C^{\prime} \cup C^{\prime \prime}\right) \backslash\left(C^{\prime} \cap C^{\prime \prime}\right)$ é uma união de circuitos disjuntos, vazia quando $C^{\prime}=C^{\prime \prime}$;

- teorema de Menger para vértices: se em um grafo $(V, E)$ há pelo menos $k$ vértices no menor subconjunto $V^{\prime} \subseteq V$ tal que o subgrafo induzido por $V \backslash V^{\prime}$ não contém $(u, v)$-caminhos, então sabemos que $(V, E)$ tem $k(u, v)$-caminhos disjuntos;

- teorema de Menger para arestas: se em um grafo $(V, E)$ tem há pelo menos $k$ arestas no menor subconjunto $E^{\prime} \subseteq E$ tal que o subgrafo $\left(V, E \backslash E^{\prime}\right)$ não contém $(u, v)$-caminhos, então sabemos que $(V, E)$ tem $k(u, v)$-caminhos aresta-disjuntos;

- circuitos alternantes para emparelhamentos perfeitos: se $M$ é um emparelhamento perfeito em um grafo sem pontes, então existe algum circuito $M$-alternante nesse grafo;

- propriedade de troca: se $F^{\prime}$ e $F^{\prime \prime}$ são florestas maximais de um grafo, então existe uma ordenação $e_{1}, \ldots, e_{l}$ das arestas em $F^{\prime}$ e uma ordenação $f_{1}, \ldots, f_{l}$ das arestas em $F^{\prime \prime}$ tal que o conjunto $\left(F^{\prime} \backslash\left\{e_{1}, \ldots, e_{i}\right\}\right) \cup\left\{f_{1}, \ldots, f_{i}\right\}$ também é uma floresta maximal para todo $i$.

Por fim, um pouco de terminologia para multiconjuntos: vamos dizer que um multiconjunto contém uma ou mais cópias de cada tipo. Quando nos referirmos a elementos distintos de um multiconjunto, estaremos considerando cópias de tipos distintos ou cópias distintas de um mesmo tipo. O sinal de igualdade =, inclusive, só será utilizado para a mesma cópia do mesmo tipo. 



\section{Capítulo 1}

\section{Sistemas e fechos lineares}

Neste primeiro capítulo, vamos apresentar o problema de obter uma solução para um sistema de equações lineares e mostrar como o algoritmo conhecido como eliminação gaussiana pode ser utilizado para resolvê-lo em tempo polinomial. Também mostraremos como o mesmo problema pode ser interpretado sob o ponto de vista de fechos lineares. Terminamos este capítulo com um "teorema de alternativas" para a existência de solução de sistemas lineares.

Os resultados apresentados neste capítulo podem ser considerados o "alicerce" deste trabalho, pois o foco dos capítulos posteriores será obter versões análogas desses resultados em outros contextos.

\subsection{Método da eliminação gaussiana}

Suponha que, dados racionais $\alpha_{1}, \ldots, \alpha_{m}$ e $\beta$, queremos obter racionais $\xi_{1}, \ldots, \xi_{m}$ que satisfaçam

$$
\alpha_{1} \xi_{1}+\cdots+\alpha_{m} \xi_{m}=\beta
$$

Uma restrição nessa forma é chamada de equação linear e valores $\xi_{1}, \ldots, \xi_{m}$ que a satisfazem são chamados de solução dessa equação.

Nem toda equação linear admite solução. Suponha, por exemplo, que $\alpha_{1}, \ldots, \alpha_{m}=0$ e $\beta \neq 0$. Nesse caso, a soma $\alpha_{1} \xi_{1}+\cdots+\alpha_{m} \xi_{m}$ é igual a zero para quaisquer $\xi_{1}, \ldots, \xi_{m}$. Logo, não pode existir solução para (1.1), visto que $\beta$ não é igual a zero.

Esse exemplo, porém, corresponde ao único caso em que uma equação linear não admite solução. Se $\alpha_{1}, \ldots, \alpha_{m}=0$ e $\beta=0$, então quaisquer racionais $\xi_{1}, \ldots, \xi_{m}$ satisfazem (1.1). Se $\alpha_{j} \neq 0$ para algum $j$, então essa equação linear é satisfeita por $\xi_{1}, \ldots, \xi_{m}$ obtidos da seguinte maneira:

$$
\xi_{i}:= \begin{cases}\alpha_{i}^{-1} \beta & \text { se } i=j \\ 0 & \text { se } i \neq j\end{cases}
$$

Resolver uma equação linear significa verificar se ela admite solução e obter uma em caso positivo. A partir das observações anteriores, podemos descrever um algoritmo polinomial para resolver uma dada equação linear.

Vamos considerar agora uma generalização desse problema: obter racionais que satisfaçam várias equações lineares simultaneamente. Mais especificamente, gostaríamos de obter racionais $\xi_{1}, \ldots, \xi_{m}$ 
para os quais vale o conjunto de igualdades

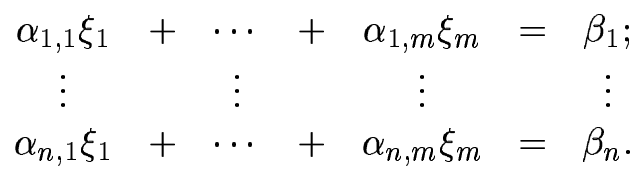

Essas igualdades podem ser descritas de maneira mais sucinta em termos de matrizes e vetores. Considere uma matriz racional $A$, um vetor racional $b$ e um vetor racional $x$ definidos por

$$
A:=\left[\begin{array}{ccc}
\alpha_{1,1} & \cdots & \alpha_{1, m} \\
\vdots & \vdots & \vdots \\
\alpha_{n, 1} & \cdots & \alpha_{n, m}
\end{array}\right], \quad b:=\left(\begin{array}{c}
\beta_{1} \\
\vdots \\
\beta_{n}
\end{array}\right), \quad x:=\left(\begin{array}{c}
\xi_{1} \\
\vdots \\
\xi_{m}
\end{array}\right)
$$

Temos nesse caso que $\xi_{1}, \ldots, \xi_{m}$ satisfazem as equações em (1.3) se e somente se o vetor $x$ satisfaz

$$
A x=b .
$$

Uma restrição nessa forma é chamada de sistema de equações lineares ou simplesmente de sistema linear. Um vetor $x$ que a satisfaz é chamado de solução desse sistema.

Resolver um sistema linear significa verificar se ele admite solução e obter uma em caso positivo. Se $A=0$, a resolução de (1.4) é análoga à resolução da equação (1.1) no caso em que $\alpha_{1}, \ldots, \alpha_{m}=0$ : não existe solução se $b \neq 0$ e qualquer vetor é solução se $b=0$.

Vamos desenvolver essa analogia em relação a equações lineares e verificar se a construção (1.2) pode ser estendida para sistemas. A idéia principal dessa construção consiste em utilizar o inverso de algum elemento dentre $\alpha_{1}, \ldots, \alpha_{m}$. Considerando o sistema linear (1.4), uma extensão natural dessa idéia é utilizar a inversa de uma submatriz de $A$. Em particular, se $A$ é uma matriz na forma

$$
\left[A_{1} A_{2}\right] \text {, onde } A_{1} \text { é uma matriz não-singular, }
$$

então podemos verificar que o sistema é satisfeito por um vetor $x$ obtido da seguinte maneira:

$$
x:=\left(\begin{array}{c}
A_{1}^{-1} b \\
0
\end{array}\right)
$$

Portanto, esse caso particular de sistema linear pode ser resolvido através do cálculo de uma matriz inversa. Se considerarmos um caso ainda mais particular, no qual a matriz $A$ está na forma

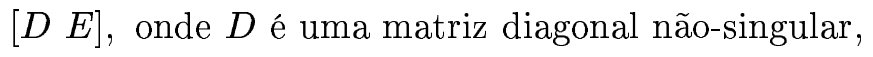

então essa inversa pode ser computada de maneira muito simples: sendo $\delta_{i, j}$ o elemento na linha $i$ e coluna $j$ de $D$, sabemos que para a inversa de $D$ vale a igualdade

$$
D^{-1}=\operatorname{diag}\left(\delta_{1,1}^{-1}, \delta_{2,2}^{-1}, \ldots, \delta_{n, n}^{-1}\right)
$$

onde $\operatorname{diag}\left(\alpha_{1}, \ldots, \alpha_{n}\right)$ é uma matriz quadrada cujo elemento na linha $i$ e coluna $j$ é $\alpha_{i}=\alpha_{j}$ se $i=j$ e zero caso contrário.

Como nem toda matriz está nessa forma restrita, tais construções não podem ser diretamente aplicadas sobre sistemas arbitrários. Entretanto, elas nos permitem considerar a estratégia de reduzir 
um dado sistema a esse caso particular. Vamos apresentar a seguir um algoritmo em duas fases para "diagonalizar" uma matriz arbitrária através de operações elementares sobre linhas e colunas.

A primeira fase desse algoritmo consiste em "triangularizar" a matriz desejada. Mais formalmente, consiste em transformar uma dada matriz $A$, de forma arbitrária, em uma matriz na forma

$$
\left[\begin{array}{cc}
T & B \\
0 & 0
\end{array}\right] \text {, onde } T \text { é uma matriz triangular superior não-singular. }
$$

Essa transformação é feita através de uma seqüência finita de operações elementares sobre as linhas e colunas de $A$. Para descrever tais operações, utilizaremos a seguinte definição: dizemos que uma matriz está na forma $T_{k}$ se essa matriz é dada por

$$
\left[\begin{array}{ll}
T & B \\
0 & C
\end{array}\right] \text {, onde } T \text { é uma matriz triangular superior não-singular de ordem } k \text {. }
$$

Como essa definição não impõe nenhuma restrição sobre a matriz $C$, segue que qualquer matriz, em particular a dada matriz $A$, está na forma $T_{0}$. Ademais, se uma matriz em $\mathbb{R}^{n \times m}$ está na forma $T_{k}$ e satisfaz $k=n$ ou $k=m$, então essa matriz está na forma (1.5). Logo, para obter a transformação desejada, é suficiente um procedimento que, dada uma matriz na forma $T_{k}$, verifica se ela está na forma (1.5) e, em caso negativo, a transforma em uma matriz na forma $T_{k+1}$ através de uma seqüência finita de operações elementares sobre linhas e colunas.

Seja $A$ uma matriz na forma $T_{k}$. Vamos denotar por $\alpha_{i, j}$ o elemento na linha $i$ e coluna $j$ de $A$ e vamos denotar por $a_{i}$ a linha $i$ de $A$. Suponha que não existem $i, j>k$ tais que $\alpha_{i, j} \neq 0$. Temos nesse caso que $C=0$ e, portanto, que $A$ está na forma (1.5). Suponha então que tais $i, j$ existem e considere as seguintes operações: se $i>k+1$, permutamos as linhas $k+1$ e $i$ de $A$; se $j>k+1$, permutamos as colunas $k+1$ e $j$ de $A$. Como tais operações não envolvem as $k$ primeiras linhas nem as $k$ primeiras colunas, a matriz permanece na forma $T_{k}$.

Ademais, após essas operações, temos $\alpha_{k+1, k+1} \neq 0$. Disso segue que, para todo $i>k+1$, vale:

(i) se $j<k$, então $\alpha_{i, j}-\lambda \alpha_{k+1, j}=0$ para todo $\lambda$, pois $\alpha_{i, j}=0$ e $\alpha_{k+1, j}=0$;

(ii) $\alpha_{i, k+1}-\lambda \alpha_{k+1, k+1}=0$ se $\lambda=\alpha_{i, k+1} / \alpha_{k+1, k+1}$.

Portanto, se para cada $i>k+1$ subtrairmos $\left(\alpha_{i, k+1} / \alpha_{k+1, k+1}\right) a_{k+1}$ de $a_{i}$, obtemos uma matriz na forma $T_{k+1}$. Isso define o procedimento desejado. O pseudo-código abaixo descreve um algoritmo que transforma uma matriz $A \in \mathbb{R}^{n \times m}$ em uma matriz na forma (1.5) e devolve a ordem de $T$.

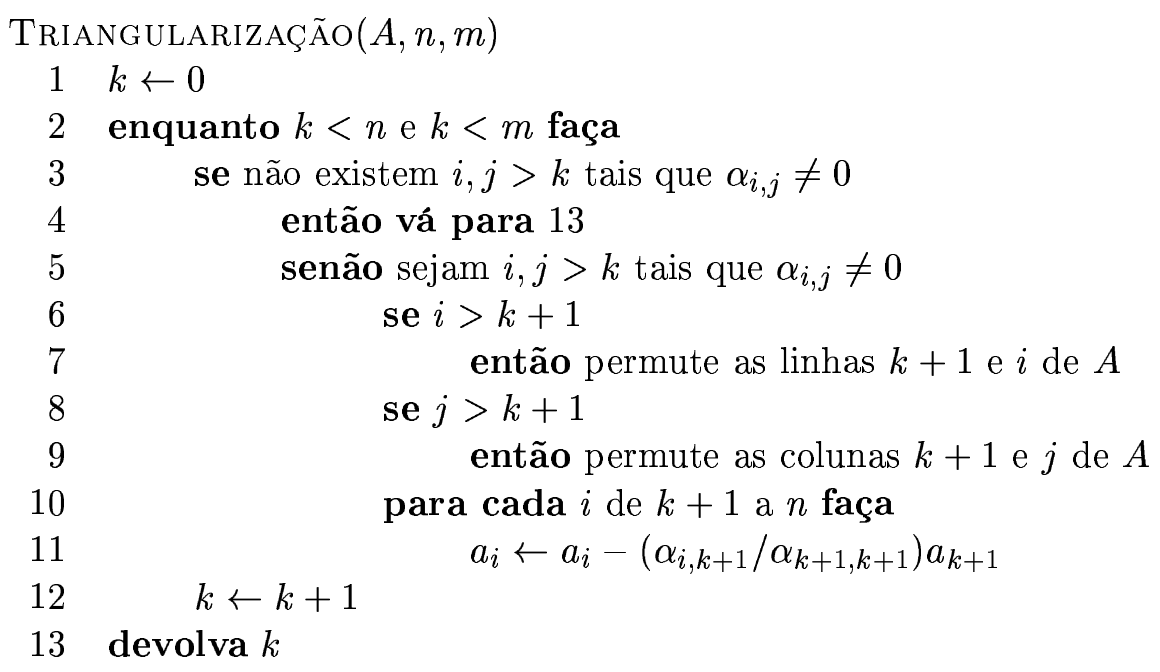


Cabe mencionar que todas as operações elementares efetuadas pelo algoritmo mantêm a matriz $T$ não-singular.

$\mathrm{Na}$ segunda fase completamos a diagonalização, a partir de uma matriz triangularizada pela primeira fase. Mais formalmente, transformamos uma matriz na forma (1.5) em uma matriz

$$
\left[\begin{array}{cc}
D & E \\
0 & 0
\end{array}\right] \text {, onde } D \text { é uma matriz diagonal não-singular. }
$$

De forma análoga à da primeira fase, essa transformação é feita através de uma seqüência finita de operações elementares sobre $A$, mas nesta segunda fase só são necessárias operações sobre linhas. Novamente, vamos definir uma forma especial de matriz para descrever as operações: dizemos que uma matriz está na forma $D_{r, k}$ se essa matriz é dada por

$$
\left[\begin{array}{ccc}
T & 0 & F \\
0 & D & E \\
0 & 0 & 0
\end{array}\right] \text {, onde }\left\{\begin{array}{l}
T \text { é uma matriz triangular superior não-singular de ordem } k \\
D \text { é uma matriz diagonal não-singular de ordem } r-k .
\end{array}\right.
$$

Como essa definição não impõe nenhuma restrição sobre a matriz $F$, segue que qualquer matriz na forma (1.5), em particular uma matriz obtida após a execução de TrIANGUlARIZAÇÃo, está na forma $D_{r, r}$, onde $r$ é a ordem de $T$. Ademais, se uma matriz está na forma $D_{r, k}$ e satisfaz $k=0$, então essa matriz está na forma (1.6). Logo, para obter a transformação desejada, é suficiente um procedimento que, dada uma matriz na forma $D_{r, k}$ transforma essa matriz em uma matriz na forma $D_{r, k-1}$ através de uma seqüência finita de operações elementares sobre linhas.

Seja $A$ uma matriz na forma $D_{r, k}$. Novamente, vamos denotar por $\alpha_{i, j}$ o elemento na linha $i$ e coluna $j$ de $A$ e vamos denotar por $a_{i}$ a linha $i$ de $A$. Como $T$ é não-singular, sabemos que $\alpha_{k, k} \neq 0$. Disso segue que, para todo $i<k$, valem as seguintes propriedades:

(i) se $k<j \leq r$, então $\alpha_{i, j}-\lambda \alpha_{k, j}=0$ para todo $\lambda$;

(ii) $\alpha_{i, k}-\lambda \alpha_{k, k}=0$ se $\lambda=\alpha_{i, k} / \alpha_{k, k}$.

Portanto, se para cada $i<k$ subtrairmos o vetor $\left(\alpha_{i, k} / \alpha_{k, k}\right) a_{k}$ de $a_{i}$, obtemos uma matriz na forma $D_{r, k-1}$. Isso define o procedimento desejado, pois tal operação preserva a não-singularidade da matriz. O pseudo-código abaixo descreve um algoritmo que transforma uma matriz na forma (1.5) em uma matriz na forma (1.6), dada a ordem $r$ de $T$.

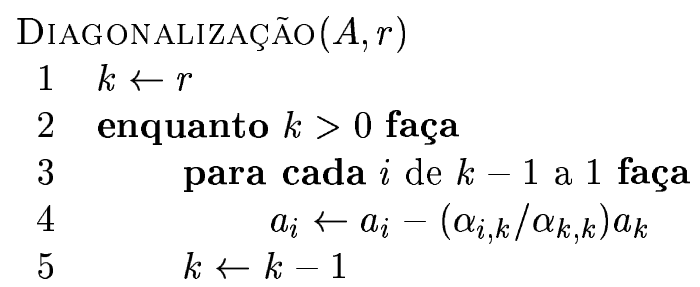

Concatenando as duas fases, podemos colocar qualquer matriz na forma (1.6) com operações elementares. Informalmente, podemos afirmar que a primeira fase consiste em "eliminar elementos abaixo da diagonal" e a segunda fase consiste em "eliminar elementos acima da diagonal". Essa maneira de interpretar o algoritmo, que foi apresentado por Gauss, deixa evidente a razão pela qual ele é conhecido como eliminação gaussiana. Vamos apresentar a seguir um método para resolver sistemas lineares através da eliminação gaussiana, além de análises sobre sua complexidade. 


\subsection{Correção da eliminação gaussiana}

Ao longo da eliminação gaussiana, são executadas sobre a matriz $A$ apenas operações elementares sobre linhas e colunas. Sabemos que cada uma dessas operações equivale à multiplicação por uma matriz não-singular. Mais especificamente, a matriz obtida no final da eliminação é dada por

$$
L_{l} \ldots L_{1} A Q_{1} \ldots Q_{q}
$$

onde $L_{1}, \ldots, L_{l}$ são matrizes não-singulares que representam, em ordem de execução, as operações elementares sobre linhas e $Q_{1}, \ldots, Q_{q}$ são matrizes não-singulares que representam, também em ordem de execução, as operações elementares sobre colunas.

Embora as matrizes $Q_{1}, \ldots, Q_{q}$ representem apenas um tipo de operação, a permutação de colunas, o mesmo não se aplica às matrizes $L_{1}, \ldots, L_{l}$, que representam tanto permutações de linhas como somas de linhas. Vamos separar esses dois tipos de operação mais formalmente, denotando por $L_{1}^{\prime}, \ldots, L_{p}^{\prime}$ as matrizes que representam as permutações de linhas, em ordem de execução, e por $L_{1}^{\prime \prime}, \ldots, L_{s}^{\prime \prime}$ as matrizes que representam as somas de linhas, em ordem de execução.

Considere a seqüência de somas feita em uma iteração da triangulação: para cada $i>k+1$, subtraímos $\left(\alpha_{i, k+1} / \alpha_{k+1, k+1}\right) a_{k+1}$ da linha $i$. Suponha que, em uma iteração posterior, as linhas $i_{1}$ e $i_{j}$ são permutadas. Pela ordem das iterações, sabemos que $i_{1}, i_{2}>k+1$. Portanto, se executarmos sobre uma matriz essa seqüência de somas e depois essa permutação, obtemos o mesmo resultado que obteríamos caso executássemos primeiro a permutação e depois a seqüência de somas. Logo,

$$
L_{l} \ldots L_{1}=L_{1} \ldots L_{1} I=L_{s}^{\prime \prime} \ldots L_{1}^{\prime \prime} L_{p}^{\prime} \ldots L_{1}^{\prime} I=L_{s}^{\prime \prime} \ldots L_{1}^{\prime \prime} L_{p}^{\prime} \ldots L_{1}^{\prime},
$$

onde $I$ é a matriz identidade. Assim, o resultado da operação

$$
L_{s}^{\prime \prime} \ldots L_{1}^{\prime \prime} L_{p}^{\prime} \ldots L_{1}^{\prime} A Q_{1} \ldots Q_{q}
$$

corresponde à matriz obtida no final da eliminação gaussiana. Vamos denotar por $S$ a matriz dada por $L_{s}^{\prime \prime} \ldots L_{1}^{\prime \prime}$, por $P$ a matriz dada por $L_{p}^{\prime} \ldots L_{1}^{\prime}$ e por $Q$ a matriz dada por $Q_{1} \ldots Q_{q}$. Segue que

$$
S P A Q=\left[\begin{array}{cc}
D & E \\
0 & 0
\end{array}\right] \text {, onde } D \text { é uma matriz diagonal não-singular. }
$$

A partir do fato de que $S, P, Q$ são matrizes não-singulares, e portanto inversíveis, podemos deduzir a equivalência que nos permite utilizar a eliminação gaussiana para resolver sistemas lineares.

Lema 1.1. Sejam $A, S, P, Q$ matrizes e seja $b$ um vetor. Suponha que $S, P, Q$ são não-singulares. Existe um vetor $x$ tal que $A x=b$ se e somente se existe um vetor $x^{\prime}$ tal que $S P A Q x^{\prime}=S P b$.

Demonstração. Se $x$ satisfaz $A x=b$, então $x$ também satisfaz $S P A x=S P b$. Ademais, temos

$$
S P A x=S P A I x=S P A Q Q^{-1} x .
$$

Podemos concluir então que, se $x$ satisfaz $A x=b$, então $x^{\prime}:=Q^{-1} x$ satisfaz $S P A Q x^{\prime}=S P b$.

Suponha agora que $x^{\prime}$ satisfaz $S P A Q x^{\prime}=S P b$. Nesse caso, $x:=Q x^{\prime}$ satisfaz $S P A x=S P b$. Como as matrizes $S, P$ são não-singulares, temos que $S P$ também é não-singular. Podemos concluir então que $x$ satisfaz $(S P)^{-1} S P A x=(S P)^{-1} S P b$ e, portanto, que $x$ satisfaz o sistema $A x=b$. 
O problema de resolver $A x=b$ pode então ser reduzido ao problema de resolver $S P A Q x^{\prime}=S P b$ : um sistema admite solução se e somente se o outro admite e, para obter uma solução de $A x=b$ a partir de uma solução de $S P A Q x^{\prime}=S P b$, basta uma multiplicação pela matriz $Q$.

Vamos então descrever como resolver o sistema $S P A Q x^{\prime}=S P b$. Seja $r$ a ordem da matriz diagonal não-singular $D$ descrita em (1.7). Sejam $c, d$ os vetores tais que a dimensão de $c$ é $r$ e

$$
S P b=\left(\begin{array}{c}
c \\
d
\end{array}\right)
$$

Podemos verificar que, se $d=0$ e $r>0$, o sistema $S P A Q x^{\prime}=S P b$ é satisfeito pela construção

$$
x^{\prime}:=\left(\begin{array}{c}
D^{-1} c \\
0
\end{array}\right)
$$

Cabe observar que trata-se precisamente da construção que motivou a diagonalização. Para o caso particular em que $d=0$ e $r=0$, obtemos o sistema $0 x^{\prime}=0$, que é satisfeito por qualquer $x^{\prime}$.

Resta, portanto, considerar o caso em que $d \neq 0$. Como a dimensão de $c$ é $r$, essa hipótese equivale a afirmar que existe $i>r$ tal que o resultado da multiplicação de $b$ pela linha $i$ de $S P$ é não-nulo. Vamos denotar essa linha de $S P$ por $y$. Sabemos a partir de (1.7) que $y A Q=0$. Logo,

$$
y A=y A Q Q^{-1}=0 Q^{-1}=0 .
$$

Podemos então concluir que, se $d \neq 0$, então existe um vetor $y$ tal que $y A=0$ e $y b \neq 0$. O lema a seguir prova que tal vetor é um certificado de que não existe solução para $A x=b$ nesse caso.

Lema 1.2. Seja $A$ uma matriz e seja $b$ um vetor. Se existe um vetor y tal que $y A=0$ e yb $\neq 0$, então não existe um vetor $x$ tal que $A x=b$.

Demonstração. Se tal $x$ existisse teríamos a igualdade $0=0 x=y A x=y b \neq 0$, uma contradição.

Temos então que um vetor $x$ satisfazendo $A x=b$ e um vetor $y$ satisfazendo $y A=0$ e $y b \neq 0$ não podem existir simultaneamente. Por outro lado, a eliminação gaussiana prova que um dos dois existe. Temos então a caracterização conhecida como teorema fundamental da álgebra linear.

Teorema 1.3. Seja $A$ uma matriz e seja $b$ um vetor. Existe um vetor $x$ tal que $A x=b$ se e somente se não existe um vetor y tal que $y A=0$ e $y b \neq 0$.

Uma conseqüência interessante do ponto de vista algorítmico é o fato de que a partir desse teorema podemos caracterizar a existência e a não-existência de solução através de um vetor.

Para concluirmos a descrição do algoritmo, ainda resta mostrar como obter as matrizes $S, P, Q$. Para atingirmos esse objetivo, no entanto, basta utilizarmos as simples igualdades a seguir:

$$
\begin{aligned}
& S=S I=L_{s}^{\prime \prime} \ldots L_{1}^{\prime \prime} I \\
& P=P I=L_{p}^{\prime} \ldots L_{1}^{\prime} I \\
& Q=I Q=I Q_{1} \ldots Q_{q} .
\end{aligned}
$$

Dessas igualdades segue que podemos calcular $S, P, Q$ ao longo da própria eliminação gaussiana: basta aplicar as mesmas operações elementares feitas sobre $A$ em matrizes definidas inicialmente como matrizes identidade. No pseudo-código dado a seguir, denotamos a linha $i$ de $S$ por $s_{i}$. 


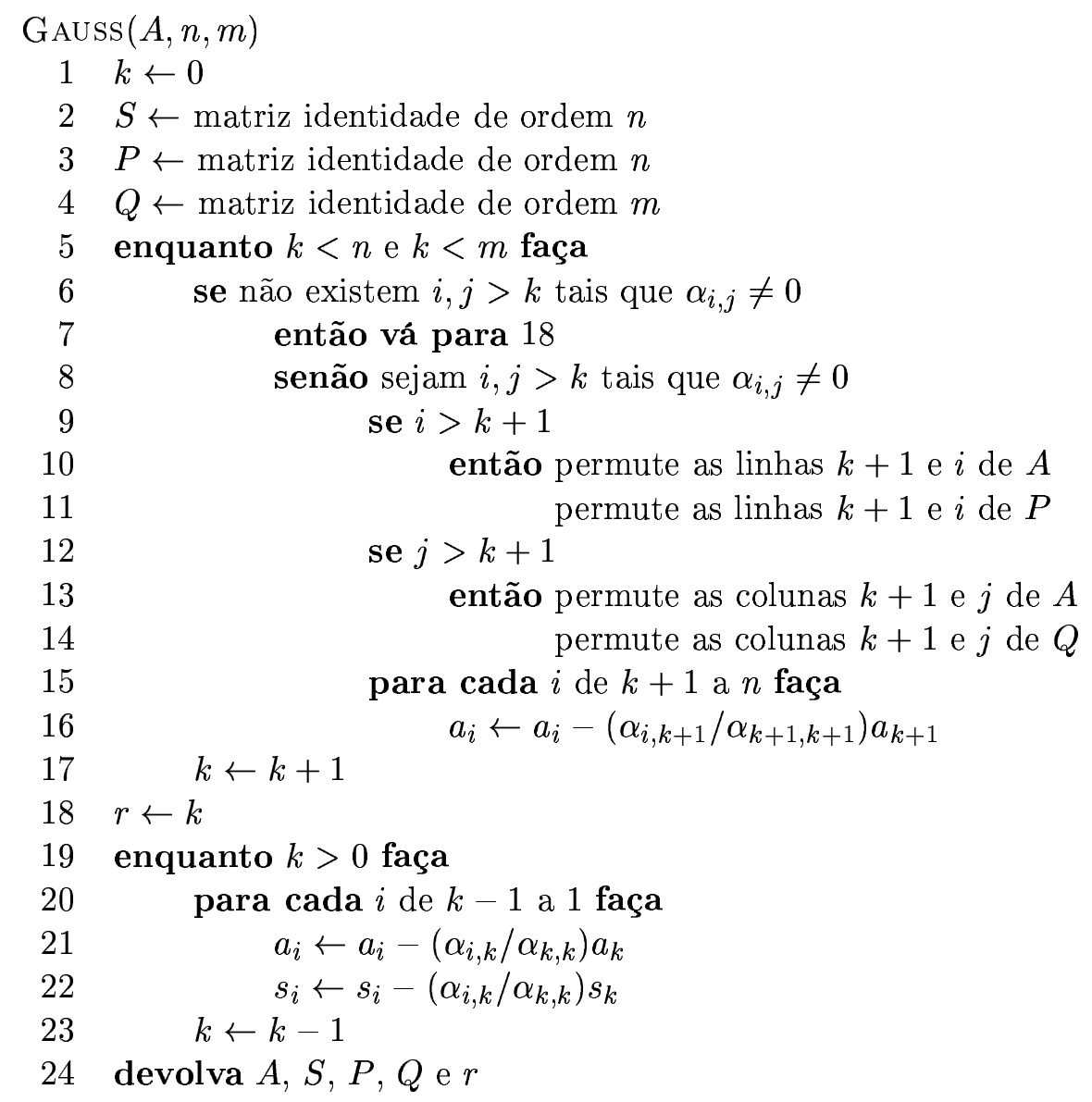

Na seção a seguir, vamos demonstrar que a eliminação gaussiana é um algoritmo polinomial.

\subsection{Eficiência da eliminação gaussiana}

A partir das linhas 18 e 24 do algoritmo Gauss, podemos deduzir que, ao longo da eliminação gaussiana, não mais do que $\min (n, m)$ iterações dos laços definidos pelas linhas 5 e 20 são executadas. Também podemos verificar que no máximo $n$ iterações do laço definido pela linha 15 são executadas para cada valor fixo de $k$. Por fim, sabemos que operações elementares consomem tempo polinomial. Para concluir que a eliminação gaussiana é um algoritmo polinomial, precisamos demonstrar que o tamanho das matrizes computadas ao longo do algoritmo é polinomialmente limitado pelo tamanho da original.

Vamos apresentar a seguir uma demonstração desse limitante, apresentada por EDMONDS [1967]. Primeiramente, vamos considerar as matrizes computadas ao longo de TrIAngulaÇÃo. A idéia da demonstração consiste em limitar o tamanho das matrizes pelo tamanho de subdeterminantes da matriz original. O primeiro passo, portanto, é limitar o tamanho desses subdeterminantes.

Teorema 1.4. Seja A uma matriz quadrada. Se A é racional, então o tamanho do determinante de A é polinomialmente limitado pelo tamanho de $A$.

Demonstração. Vamos denotar por $\alpha_{i, j}$ o elemento na linha $i$ e coluna $j$ de $A$. Sendo $\alpha_{i, j}$ racional para quaisquer $i, j$, podemos também denotar por $\mu_{i, j}$ e $\delta_{i, j}$ os dois inteiros relativamente primos 
tais que $\alpha_{i, j}=\mu_{i, j} / \delta_{i, j}$ e $\delta_{i, j}>0$. Por fim, como da fórmula de Laplace segue que $\operatorname{det}(A)$ é racional, podemos denotar por $\mu$ e $\delta$ os inteiros relativamente primos tais que $\operatorname{det}(A)=\mu / \delta$ e $\delta>0$.

Por definição, sabemos que $\operatorname{tam}(\operatorname{det}(A))=\lceil\log (|\mu|+1)\rceil+\lceil\log (\delta+1)\rceil+1$. Vamos então obter limitantes para $|\mu|$ e $\delta$. Para tanto, podemos utilizar a fórmula de Laplace, pois dela segue que $|\operatorname{det}(A)| \leq \prod_{i, j}\left(\left|\alpha_{i, j}\right|+1\right)$ e, portanto, que $|\operatorname{det}(A)| \leq \prod_{i, j}\left(\left|\mu_{i, j}\right|+1\right)$ e $\delta \leq \prod_{i, j} \delta_{i, j}$. Logo,

$$
\log (\delta) \leq \log \left(\prod_{i, j} \delta_{i, j}\right)=\sum_{i, j} \log \left(\delta_{i, j}\right) \leq \sum_{i, j} \operatorname{tam}\left(\alpha_{i, j}\right)=\operatorname{tam}(A)-m-n \leq \operatorname{tam}(A)-1
$$

e, como $|\operatorname{det}(A)|=|\mu| / \delta$, temos que um limitante para $|\mu|$ pode ser calculado de maneira análoga:

$$
\begin{aligned}
\log (|\mu|) \leq & \log \left(\prod_{i, j}\left(\left|\mu_{i, j}\right|+1\right) \prod_{i, j} \delta_{i, j}\right)=\sum_{i, j} \log \left(\left|\mu_{i, j}\right|+1\right)+\sum_{i, j} \log \left(\delta_{i, j}\right)= \\
& \sum_{i, j}\left(\log \left(\left|\mu_{i, j}\right|+1\right)+\log \left(\delta_{i, j}\right)\right) \leq \sum_{i, j} \operatorname{tam}\left(\alpha_{i, j}\right)=\operatorname{tam}(A)-m-n \leq \operatorname{tam}(A)-1 .
\end{aligned}
$$

Podemos então aplicar a definição de $\operatorname{tam}(\operatorname{det}(A))$ mencionada para obter o limitante desejado:

$$
\begin{aligned}
\operatorname{tam}(\operatorname{det}(A)) & =\lceil\log (|\mu|+1)\rceil+\lceil\log (\delta+1)\rceil+1 \\
& \leq\left\lceil\log \left(2^{\operatorname{tam}(A)-1}+1\right)\right\rceil+\left\lceil\log \left(2^{\operatorname{tam}(A)-1}+1\right)\right\rceil+1 \\
& \leq\left\lceil\log \left(2 \cdot 2^{\operatorname{tam}(A)-1}\right)\right\rceil+\left\lceil\log \left(2 \cdot 2^{\operatorname{tam}(A)-1}\right)\right\rceil+1 \\
& =\left\lceil\log \left(2^{\operatorname{tam}(A)}\right)\right\rceil+\left\lceil\log \left(2^{\operatorname{tam}(A)}\right)\right\rceil+1 \\
& =\lceil\operatorname{tam}(A)\rceil+\lceil\operatorname{tam}(A)\rceil+1 \\
& =2 \operatorname{tam}(A)+1 .
\end{aligned}
$$

Como conseqüência desse teorema, podemos limitar também o tamanho da matriz inversa.

Corolário 1.5. Seja $A$ uma matriz quadrada. Se A é racional, então o tamanho da inversa $A^{-1} e^{-}$ polinomialmente limitado pelo tamanho de $A$.

Demonstração. Segue do teorema 1.4 e do fato de que o elemento na linha $i$ e coluna $j$ de $A^{-1}$ é

$$
\frac{(-1)^{i+j} \operatorname{det}\left(A_{i, j}\right)}{\operatorname{det}(A)} \text {, onde } A_{i, j} \text { é uma submatriz de } A \text {. }
$$

Esses dois limitantes podem ser utilizados para limitar o tamanho das matrizes obtidas ao longo do procedimento de triangulação da eliminação gaussiana, como demonstrado no resultado a seguir.

Lema 1.6. Ao longo de todo o procedimento de triangulação do algoritmo GAUss, o tamanho das matrizes consideradas é polinomialmente limitado pelo tamanho de A. 
Demonstração. Como vimos anteriormente, sabemos que, no final de cada iteração da triangulação,

$$
S P A Q=\left[\begin{array}{ll}
T & B \\
0 & C
\end{array}\right] \text {, onde } T \text { é uma matriz triangular superior não-singular de ordem } k \text {. }
$$

Sabemos também que $S$ é igual a $S I$, sendo $I$ é a matriz identidade. Podemos considerar então uma partição dessa matriz identidade:

$$
S=S\left[\begin{array}{cc}
I_{1} & 0 \\
0 & I_{2}
\end{array}\right], \text { onde } I_{1} \text { e } I_{2} \text { são matrizes identidade. }
$$

Em particular, podemos supor também que a ordem de $I_{1}$ é $k$. Como a multiplicação por $S$ equivale a uma seqüência de somas das $k$ primeiras linhas às outras, essa partição nos ajuda a verificar que

$$
S=\left[\begin{array}{ll}
S_{1} & 0 \\
S_{2} & I
\end{array}\right] \text {, onde } S_{1} \text { é uma matriz quadrada de ordem } k \text {. }
$$

visto que $S$ é uma matriz não-singular. Estendendo essa estratégia de particionar, vamos considerar

$$
A=\left[\begin{array}{ll}
A_{1} & A_{2} \\
A_{3} & A_{4}
\end{array}\right] \text {, onde } A_{1} \text { é uma matriz quadrada de ordem } k .
$$

Como $S$ é uma matriz não-singular, temos que $S_{1}$ é não-singular. Ademais, como $S_{1} A_{1}=T$, temos que $A_{1}=S_{1}^{-1} T$ e, portanto, que $A_{1}$ também é não-singular. Isso nos permite concluir que $S_{1}=T A_{1}^{-1}$. Novamente utilizando a partição, também podemos verificar que $S_{2} A_{1}+I A_{3}=0$. Como vimos que $A_{1}$ é não-singular, disso segue que $S_{2}=-A_{3} A_{1}^{-1}$.

Seja $\gamma$ um elemento da submatriz $C$, mais especificamente o elemento na linha $i$ e coluna $j$ da matriz $S P A Q$. A partir desse elemento, vamos considerar a seguinte submatriz de $S P A Q$ :

$$
T^{\prime}=\left[\begin{array}{ll}
T & b \\
0 & \gamma
\end{array}\right], \text { onde } b \text { é a coluna de } B \text { correspondente à coluna } j \text { de } S P A Q .
$$

Seja $\alpha$ o elemento na linha $i$ e coluna $j$ de $P A Q$. Podemos considerar, analogamente, a submatriz

$$
A_{1}^{\prime}=\left[\begin{array}{cc}
A_{1} & a_{2} \\
a_{3} & \alpha
\end{array}\right], \text { onde }\left\{\begin{array}{l}
a_{2} \text { é a coluna de } A_{2} \text { correspondente à coluna } j \text { de } P A Q ; \\
a_{3} \text { é a linha de } A_{3} \text { correspondente à linha } i \text { de } P A Q .
\end{array}\right.
$$

Podemos então verificar que existe uma matriz $S_{1}^{\prime}$ tal que $S^{\prime} A_{1}^{\prime}=T^{\prime}$. Mais especificamente,

$$
S_{1}^{\prime}=\left[\begin{array}{ll}
S_{1} & 0 \\
s_{2} & 1
\end{array}\right], \text { onde } s_{2} \text { é a linha de } S_{2} \text { correspondente à linha } i \text { de } S \text {. }
$$

Pela fórmula de Laplace, temos que $\left|\operatorname{det}\left(T^{\prime}\right)\right|=|\gamma \operatorname{det}(T)| . \operatorname{Logo},|\gamma|=\left|\operatorname{det}\left(S_{1}^{\prime} A_{1}^{\prime}\right)\right| /\left|\operatorname{det}\left(S_{1} A_{1}\right)\right|$. Como temos $\operatorname{det}(S)=1$, segue que $\operatorname{det}\left(S_{1}\right)=1 \mathrm{e} \operatorname{det}\left(S_{1}^{\prime}\right)=1 . \mathrm{Assim},|\gamma|=\left|\operatorname{det}\left(A_{1}^{\prime}\right)\right| /\left|\operatorname{det}\left(A_{1}\right)\right|$. Como $A_{1}^{\prime}$ e $A_{1}$ são submatrizes de $P A Q$, sabemos que todos os elementos dessas matrizes são elementos de $A$. Logo, pelo teorema 1.4, o tamanho de $\gamma$ é polinomialmente limitado pelo tamanho de $A$. Portanto, o tamanho da matriz $C$ é polinomialmente limitado pelo tamanho de $A$.

Como todo elemento não-nulo de $T$ e $B$ é elemento da matriz $C$ correspondente a alguma iteração anterior da triangulação, podemos concluir que ao longo de toda o procedimento, $\operatorname{tam}(S P A Q)$ é polinomialmente limitado por $\operatorname{tam}(A)$. Visto que $\operatorname{tam}(P)$ e $\operatorname{tam}(Q)$ também são, resta verificar o mesmo para $\operatorname{tam}(S)$. Como $S_{1}=T A_{1}^{-1}$ e $S_{2}=-A_{3} A_{1}^{-1}$, esse fato segue do corolário 1.5 . 
Vamos agora verificar a eficiência do procedimento de diagonalização da eliminação gaussiana.

Lema 1.7. Ao longo de todo o procedimento de diagonalização do algoritmo GAUss, o tamanho das matrizes consideradas é polinomialmente limitado pelo tamanho de A.

Demonstração. Sabemos que no final de cada iteração $k$ da diagonalização, temos a igualdade

$S P A Q=\left[\begin{array}{ccc}T & 0 & F \\ 0 & D & E \\ 0 & 0 & 0\end{array}\right]$, onde $\left\{\begin{array}{l}T \text { é uma matriz triangular superior não-singular de ordem } k ; \\ D \text { é uma matriz diagonal não-singular de ordem } r-k .\end{array}\right.$

Sabemos também que, no início da diagonalização, temos uma matriz que foi obtida a partir do procedimento de triangulação. Mais especificamente, podemos particionar essa matriz de acordo:

$\left[\begin{array}{ccc}T_{1} & T_{2} & B_{1} \\ 0 & T_{3} & B_{2} \\ 0 & 0 & 0\end{array}\right]$, onde $\left\{\begin{array}{l}T_{1} \text { é uma matriz triangular superior não-singular de ordem } k \\ T_{3} \text { é uma matriz triangular superior não-singular de ordem } r-k .\end{array}\right.$

Como ao longo da diagonalização são executadas apenas somas de linhas, existe uma matriz

$$
S^{\prime}=\left[\begin{array}{ccc}
S_{1}^{\prime} & S_{2}^{\prime} & S_{3}^{\prime} \\
S_{4}^{\prime} & S_{5}^{\prime} & S_{6}^{\prime} \\
S_{7}^{\prime} & S_{8}^{\prime} & S_{9}^{\prime}
\end{array}\right], \text { onde }\left\{\begin{array}{l}
S_{1}^{\prime} \text { é uma matriz quadrada de ordem } k \\
S_{5}^{\prime} \text { é uma matriz quadrada de ordem } r-k .
\end{array}\right.
$$

que, multiplicada pela matriz inicial, resulta na matriz $S P A Q$. Mais especificamente, temos que

$$
\left[\begin{array}{ccc}
T & 0 & F \\
0 & D & E \\
0 & 0 & 0
\end{array}\right]=\left[\begin{array}{ccc}
S_{1}^{\prime} T_{1}+S_{2}^{\prime} 0+S_{3}^{\prime} 0 & S_{1}^{\prime} T_{2}+S_{2}^{\prime} T_{3}+S_{3}^{\prime} 0 & S_{1}^{\prime} B_{1}+S_{2}^{\prime} B_{2}+S_{3}^{\prime} 0 \\
S_{4}^{\prime} T_{1}+S_{5}^{\prime} 0+S_{6}^{\prime} 0 & S_{4}^{\prime} T_{2}+S_{5}^{\prime} T_{3}+S_{6}^{\prime} 0 & S_{4}^{\prime} B_{1}+S_{5}^{\prime} B_{2}+S_{6}^{\prime} 0 \\
S_{7}^{\prime} T_{1}+S_{8}^{\prime} 0+S_{9}^{\prime} 0 & S_{7}^{\prime} T_{2}+S_{8}^{\prime} T_{3}+S_{9}^{\prime} 0 & S_{7}^{\prime} B_{1}+S_{8}^{\prime} B_{2}+S_{9}^{\prime} 0
\end{array}\right] .
$$

Temos então que $S_{1}^{\prime} T_{1}=T, S_{4}^{\prime} T_{1}=0$ e $S_{7}^{\prime} T_{1}=0$. Logo, $S_{1}^{\prime}=T T_{1}^{-1}, S_{4}^{\prime}=0$ e $S_{7}^{\prime}=0$. Também temos que $S_{1}^{\prime} T_{2}+S_{2}^{\prime} T_{3}=0, S_{4}^{\prime} T_{2}+S_{5}^{\prime} T_{3}=D$ e $S_{7}^{\prime} T_{2}+S_{8}^{\prime} T_{3}=0$. Logo, $S_{2}^{\prime}=-T T_{1}^{-1} T_{2} T_{3}^{-1}$, $S_{5}^{\prime}=D T_{3}^{-1}$ e $S_{8}^{\prime}=0$. Por fim, temos $S_{1}^{\prime} B_{1}+S_{2}^{\prime} B_{2}=F, S_{4}^{\prime} B_{1}+S_{5}^{\prime} B_{2}=E$ e $S_{7}^{\prime} B_{1}+S_{8}^{\prime} B_{2}=0$. Como vimos na demonstração do teorema anterior, sabemos que $S_{3}^{\prime}=0, S_{6}^{\prime}=0$ e $S_{9}^{\prime}=I$.

Como $T=T_{1}$ e os elementos da diagonal de $D$ são os elementos da diagonal de $T_{3}$, podemos enfim concluir que $\operatorname{tam}(S)$ e $\operatorname{tam}(S P A Q)$ são polinomialmente limitados pelo tamanho de $A$.

Com os dois lemas anteriores, provamos os limitantes superiores para todo o algoritmo.

Teorema 1.8. O algoritmo GAUss é polinomial.

Demonstração. Segue diretamente dos lemas 1.6 e 1.7.

Uma conseqüência interessante desse teorema é o fato de que, se por um lado sua demonstração utilizou um limitante para o tamanho do determinante de uma matriz, por outro lado ele prova que o determinante pode ser calculado em tempo polinomial.

Corolário 1.9. Existe um algoritmo polinomial que, dada uma matriz quadrada racional $A$, devolve $o$ determinante $\operatorname{det}(A)$. 
Demonstração. Seja $A$ uma matriz quadrada de ordem $n$ e sejam $S, P, Q$ as matrizes obtidas após aplicarmos o algoritmo Gauss sobre $A$. Como $\operatorname{det}(S P A Q)=\operatorname{det}(S) \operatorname{det}(P) \operatorname{det}(A) \operatorname{det}(Q)$, temos

$$
\operatorname{det}(A)=\frac{\operatorname{det}(S P A Q)}{\operatorname{det}(S) \operatorname{det}(P) \operatorname{det}(Q)} .
$$

Como $\operatorname{det}(P)$ e $\operatorname{det}(Q)$ podem ser calculados em tempo polinomial e sabemos que $\operatorname{det}(S)=1$, resta apenas mostrar como calcular $\operatorname{det}(S P A Q)$. Sabemos pela eliminação gaussiana que

$$
S P A Q=\left[\begin{array}{cc}
D & E \\
0 & 0
\end{array}\right] \text {, onde } D \text { é uma matriz diagonal não-singular. }
$$

Seja $r$ a ordem de $D$. Se $r<n$, então $S P A Q$ é uma matriz singular e $\operatorname{det}(S P A Q)=0$. Se $r=n$, então $S P A Q=D$ e $\operatorname{det}(S P A Q)$ é obtido multiplicando-se os elementos da diagonal de $D$.

Como vimos que cada elemento da matriz inversa $A^{-1}$ é igual ao quociente do determinante de uma submatriz de $A$ pelo determinante de $A$, esse corolário implica que a matriz inversa pode ser calculada em tempo polinomial. A demonstração a seguir mostra um resultado ainda mais forte: que podemos obter a matriz inversa diretamente a partir da eliminação gaussiana.

Corolário 1.10. Existe um algoritmo polinomial que, dada uma matriz quadrada racional $A$, decide se $A$ é não-singular e devolve $A^{-1}$ em caso positivo.

Demonstração. Como vimos na demonstração do corolário 1.9 , se a matriz $A$ é não-singular, então ao final de uma aplicação da eliminação gaussiana sobre $A$ temos $S P A Q=D$. Portanto, temos

$$
\begin{aligned}
S P A Q & =D \\
S P A & =D Q^{-1} \\
D^{-1} S P A & =Q^{-1} \\
Q D^{-1} S P A & =I
\end{aligned}
$$

Logo, por definição, a matriz inversa $A^{-1}$ é o resultado da multiplicação $Q D^{-1} S P$ e vimos anteriormente que a inversa $D^{-1}$ pode ser calculada em tempo polinomial.

A eliminação gaussiana é, portanto, um algoritmo que permite resolver em tempo polinomial não apenas sistemas lineares em tempo polinomial, como também outros problemas da álgebra linear.

\subsection{Fechos lineares e bases}

Ao longo de todo este trabalho, estaremos considerando escalares, vetores e matrizes racionais salvo menção em contrário. Devido a esse fato, vamos a partir deste ponto deixar subentendida a hipótese de racionalidade na formulação dos resultados para torná-las mais sucintas.

Sejam $a_{1}, \ldots, a_{m}$ vetores. O fecho linear gerado por tais vetores é

$$
\operatorname{lin}\left(a_{1}, \ldots, a_{m}\right):=\left\{a: a=\lambda_{1} a_{1}+\cdots+\lambda_{m} a_{m}, \text { onde } \lambda_{1}, \ldots, \lambda_{m} \in \mathbb{R}\right\} .
$$

Mais informalmente, é o conjunto de todos os vetores gerados por combinações lineares de $a_{1}, \ldots, a_{m}$.

Seja $A$ uma matriz tal que $a_{i}$ é a coluna $i$ de $A$. Nesse caso, podemos verificar que um dado vetor $b$ está em $\operatorname{lin}\left(a_{1}, \ldots, a_{m}\right)$ se e somente se o sistema $A x=b$ admite solução. Em caso positivo, os elementos de uma solução $x$ são os coeficientes da combinação linear correspondente. Podemos então redefinir o teorema fundamental da álgebra linear nesses termos, como demonstrado abaixo. 
Teorema 1.11. Sejam $a_{1}, \ldots, a_{m}, b$ vetores. $O$ vetor $b$ pertence ao fecho linear de $a_{1}, \ldots, a_{m}$ se $e$ somente se não existe um vetor $y$ tal que $y b \neq 0$ e ya $a_{i}=0$ para todo $i$.

Demonstração. Segue diretamente do teorema 1.3.

Como conseqüência da eliminação gaussiana, existe um algoritmo polinomial correspondente.

Teorema 1.12. Existe um algoritmo polinomial que, dados vetores $a_{1}, \ldots, a_{m}, b$, verifica se o vetor $b$ pertence ao fecho linear de $a_{1}, \ldots, a_{m}$, devolve uma combinação linear correspondente em caso positivo e devolve um vetor y tal que $y b \neq 0$ e ya $a_{i}=0$ para todo $i$ em caso negativo.

Geometricamente, um fecho linear pode ser visualizado como um plano e o teorema afirma que esse plano pode ser caracterizado por um certo conjunto de vetores ortogonais a ele. A figura 1.1 mostra um exemplo dessa interpretação: o plano indicado pela figura é gerado pelos vetores $a_{1}, a_{2}$, mas também pode ser caracterizado como sendo o conjunto de todos os vetores ortogonais a $y$.

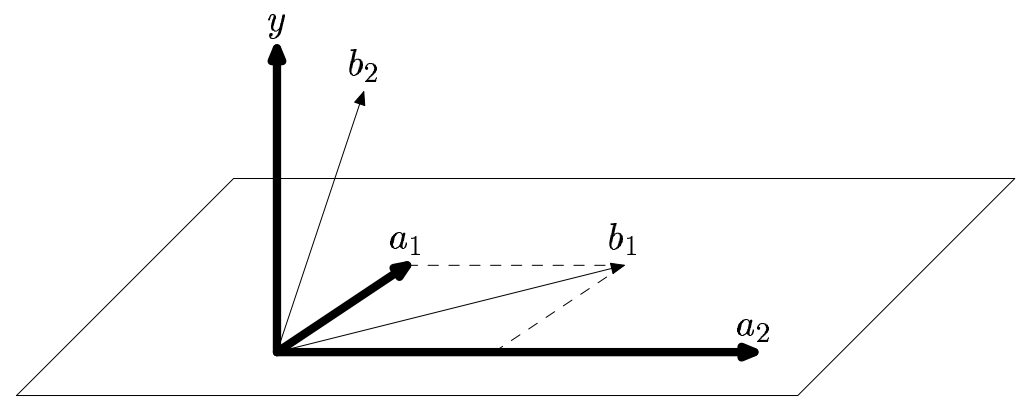

Figura 1.1: $\mathrm{O}$ vetor $b_{1}$ pertence ao fecho linear dos vetores $a_{1}, a_{2}$, mas o vetor $b_{2}$ não pertence. Como certificado de que $b_{2}$ não pertence, temos o vetor $y$, que é ortogonal a $a_{1}, a_{2}$ mas não é ortogonal a $b_{2}$.

Considere agora um conjunto $\mathcal{A}$ de vetores e um dado vetor $a$ desse conjunto. Se esse vetor é gerado por uma combinação linear dos vetores em $\mathcal{A} \backslash\{a\}$, então temos que $\operatorname{lin}(\mathcal{A} \backslash\{a\})=\operatorname{lin}(\mathcal{A})$. Logo, se $\mathcal{A}$ é linearmente dependente, então esse conjunto contém mais vetores do que o necessário para gerar $\mathcal{A}$. Por outro lado, se $\mathcal{A}$ é linearmente independente, então para qualquer $a$ em $\mathcal{A}$ vale que $\operatorname{lin}(\mathcal{A} \backslash\{a\}) \neq \operatorname{lin}(\mathcal{A})$. Mais formalmente, $\mathcal{A}$ é minimal em relação a seu fecho linear.

Dizemos que um conjunto $\mathcal{A}$ de vetores é uma base de um dado fecho linear $\mathcal{L} \operatorname{se} \operatorname{lin}(\mathcal{A})=\mathcal{L}$ e $\mathcal{A}$ é linearmente independente. A eliminação gaussiana permite obter bases em tempo polinomial.

Teorema 1.13. Existe um algoritmo polinomial que, dado um conjunto de vetores, devolve uma base do fecho linear gerado por esse conjunto.

Demonstração. Sejam $a_{1}, \ldots, a_{m}$ vetores e seja $A$ uma matriz com $m$ linhas tal que $a_{i}$ é a linha $i$ de $A$. Após a eliminação gaussiana, obtemos matrizes $S, P, Q$ para as quais vale que

$$
S P A Q=\left[\begin{array}{cc}
D & E \\
0 & 0
\end{array}\right] \text {, onde } D \text { é uma matriz diagonal não-singular. }
$$

Seja $r$ a ordem de $D$. Como vimos na demonstração do lema 1.6, podemos verificar que

$$
P A Q=\left[\begin{array}{ll}
A_{1} & A_{2} \\
A_{3} & A_{4}
\end{array}\right] \text {, onde } A_{1} \text { é uma matriz triangular superior não-singular de ordem } r \text {. }
$$


Vamos então particionar a matriz $P A$ também a partir de $r$, mas apenas em relação às linhas:

$$
P A=\left[\begin{array}{c}
A^{\prime} \\
A^{\prime \prime}
\end{array}\right], \text { onde } A^{\prime} \text { é uma matriz com } r \text { linhas. }
$$

Segue que $\left[A_{1} A_{2}\right]=A^{\prime} Q$, o que implica $A^{\prime}=\left[A_{1} A_{2}\right] Q^{-1}$. Seja $y$ um vetor tal que $y A^{\prime}=0$. Como $y\left[A_{1} A_{2}\right] Q^{-1}=0$ e $Q^{-1}$ é não-singular, temos que $y\left[A_{1} A_{2}\right]=0$. Mas como $A_{1}$ também é não-singular, podemos concluir que $y=0$. Logo, as linhas de $A^{\prime}$ são linearmente independentes.

Vamos então particionar a matriz $S$, novamente retomando o que foi feito na prova do lema 1.6.

$$
S=\left[\begin{array}{ll}
S_{1} & 0 \\
S_{2} & I
\end{array}\right] \text {, onde } S_{1} \text { é uma matriz não-singular de ordem } r \text {. }
$$

Seja $a^{\prime \prime}$ uma linha de $A^{\prime \prime}$, mais especificamente a linha $i$ de $P A$. Considere $s$ como a linha de $S_{2}$ correspondente à linha $i$ de $S$. Pela partição de $S$ e pela eliminação gaussiana, sabemos que

$$
\left(\begin{array}{ll}
s & 1
\end{array}\right)\left[\begin{array}{l}
A^{\prime} \\
a^{\prime \prime}
\end{array}\right] Q=0
$$

Como $Q$ é uma matriz não-singular, podemos concluir que $a^{\prime \prime}$ é gerado por uma combinação linear das linhas de $A^{\prime}$. Logo, o conjunto das linhas de $A^{\prime}$ é um subconjunto linearmente independente maximal do conjunto das linhas de $P A$. Portanto, após a eliminação gaussiana, um subconjunto linearmente independente de $a_{1}, \ldots, a_{m}$ é dado pelas $r$ primeiras linhas de $P A$.

Os capítulos posteriores consistem em verificar resultados análogos sob certas restrições. 



\section{Capítulo 2}

\section{Desigualdades lineares e cones}

Neste capítulo, vamos apresentar o problema de obter uma solução não-negativa para um sistema linear, o problema de obter uma solução para um sistema de desigualdades lineares e o problema de obter uma solução para um programa linear. Vamos mostrar que esses problemas são equivalentes e como são interpretados geometricamente sob o ponto de vista de cones.

As seções deste capítulo serão sucintas, pois os resultados aqui apresentados são clássicos e as demonstrações foram baseadas no livro de SCHRIJver [1986].

\subsection{Teorema das desigualdades lineares}

O teorema fundamental das desigualdades lineares, que mostraremos a seguir, é o suporte de praticamente todos os resultados deste capítulo. Ele é baseado em argumentos de FARKAS [1894] e Minkowski [1896] e em refinamentos posteriores de CARATHÉodory [1911] e WeYL [1934].

Teorema 2.1. Sejam $a_{1}, \ldots, a_{m}, b$ vetores e seja $r:=\operatorname{dim}\left(a_{1}, \ldots, a_{m}, b\right)$. Uma e somente uma dentre as afirmações a seguir é verdadeira:

(i) b é gerado por uma combinação não-negativa de vetores linearmente independentes dentre $a_{1}, \ldots, a_{m}$;

(ii) existe um vetor c tal que $c b<0, c a_{i} \geq 0$ para todo i $e\{x: c x=0\}$ contém pelo menos $r-1$ vetores linearmente independentes dentre $a_{1}, \ldots, a_{m}$.

Demonstração. As afirmações (i) e (ii) não podem simultaneamente verdadeiras: basta observar que, se isso fosse verdade, existiriam coeficientes $\lambda_{1}, \ldots, \lambda_{m} \geq 0$ satisfazendo $\lambda_{1} a_{1}+\cdots+\lambda_{m} a_{m}=b$. Isso implicaria na contradição

$$
0>c b=c\left(\lambda_{1} a_{1}+\cdots+\lambda_{m} a_{m}\right)=\lambda_{1} c a_{1}+\cdots+\lambda_{m} c a_{m} \geq 0 .
$$

Assim, vamos primeiramente mostrar que podemos supor que os vetores $a_{1}, \ldots, a_{m}$ geram todo o espaço. Seja $A$ a matriz cujas colunas são $a_{1}, \ldots, a_{m}$. Supor que as colunas geram todo o espaço equivale a $A$ ter posto linha completo.

Se $A$ não tem posto linha completo, então temos que existe uma matriz não-singular $P$ com

$$
P\left[\begin{array}{ll}
A & b
\end{array}\right]=P\left[\begin{array}{ll}
A_{1} & b_{1} \\
A_{2} & b_{2}
\end{array}\right]=\left[\begin{array}{cc}
A_{1}^{\prime} & b_{1}^{\prime} \\
0 & b_{2}^{\prime}
\end{array}\right]=\left[\begin{array}{ll}
A^{\prime} & b^{\prime}
\end{array}\right],
$$


onde $A_{1}^{\prime}$ é uma matriz com posto linha completo. Como as afirmações que queremos demonstrar são preservadas diante de quaisquer permutações feitas sobre as linhas de $[A b]$, então podemos supor sem perda de generalidade que vale $A_{1}=A_{1}^{\prime}$.

Se $b_{2}^{\prime}=0$ e a afirmação (i) vale para $\left[A_{1}^{\prime} b_{1}^{\prime}\right]$, então ela vale para $[A b]$. Analogamente, se $b_{2}^{\prime}=0$ e a afirmação (ii) vale para $\left[A_{1}^{\prime} b_{1}^{\prime}\right]$, então (ii) também vale para $[A b]$. Logo, podemos supor que existe um elemento $\beta$ de $b_{2}^{\prime} \operatorname{com} \beta \neq 0$. Sem perda de generalidade, podemos também supor $\beta<0$.

Como $\beta \neq 0$, então o sistema $A x=b$ não tem solução e, portanto, a afirmação (i) não é válida nesse caso. Vamos, então, provar que a (ii) vale.

Como pudemos verificar, existe uma linha $c$ da dada matriz $P$ para a qual vale

$$
c\left[\begin{array}{ll}
A & b
\end{array}\right]=\left[\begin{array}{ll}
0 & \beta
\end{array}\right] .
$$

Portanto, $c$ é um vetor que satisfaz as condições da afirmação (ii), visto que $\beta<0$ e que o posto de $\left[\begin{array}{ll}A & b\end{array}\right]$ não é maior do que $m+1$. Assim, provamos a afirmação e podemos supor sem perda de generalidade que $A$ tem posto linha completo.

Vamos então apresentar uma demonstração algorítmica de que (i) ou (ii) vale. Como estamos supondo que os vetores $a_{1}, \ldots, a_{m}$ geram todo o espaço, então existe um subconjunto linearmente independente $\mathcal{D}:=\left\{a_{i_{1}}, \ldots, a_{i_{n}}\right\}$ do conjunto $\left\{a_{1}, \ldots, a_{m}\right\}$, onde $n$ é a dimensão do espaço vetorial ao qual esses vetores pertencem. Considere a iteração

(1) Determine $\lambda_{i_{1}}, \ldots, \lambda_{i_{n}}$ tais que $b=\lambda_{i_{1}} a_{i_{1}}+\cdots+\lambda_{i_{n}} a_{i_{n}}$. Se $\lambda_{i_{1}}, \ldots, \lambda_{i_{n}} \geq 0$, então as condições da afirmação (i) valem e essa iteração pode terminar;

(2) Seja $h$ um índice em $i_{1}, \ldots, i_{n}$ tal que $\lambda_{h}<0$ e $h$ é o menor possível. Seja $\{x: c x=0\}$ o hiperplano gerado por $\mathcal{D} \backslash\left\{a_{h}\right\}$. Se $c a_{h} \neq 1$, divida $c$ por $c a_{h}$ e chame o novo vetor de $c$. Assim teremos que $c a_{h}=1$ e $c b=\lambda_{h}<0$;

(3) Se $c a_{1}, \ldots, c a_{m} \geq 0$, a afirmação (ii) é satisfeita, pois o posto de $a_{1}, \ldots, a_{m}, b$ é igual ao posto de $a_{1}, \ldots, a_{m}$ e a iteração pode acabar;

(4) Seja $s$ um índice em $1, \ldots, m$ tal que $c a_{s}<0$ e $s$ é o menor possível. Substitua $\mathcal{D}$ pelo conjunto $\left(\mathcal{D} \backslash\left\{a_{h}\right\}\right) \cup\left\{a_{s}\right\}$ e comece uma nova iteração.

No passo (2), como a matriz cujas colunas são os vetores em $\mathcal{D} \backslash\left\{a_{h}\right\}$ não tem posto linha completo, então existe um vetor não-nulo $c$ tal que $c a_{j}=0$ para todo $a_{j}$ em $\mathcal{D} \backslash\left\{a_{h}\right\}$, o que garante a existência do hiperplano mencionado. Ademais, como $\mathcal{D}$ é um conjunto linearmente independente, temos também que $c a_{h} \neq 0$.

A igualdade $c b=\lambda_{h}$ em (2) segue de $c b=\lambda_{1_{i}} c a_{i_{1}}+\cdots+\lambda_{1_{n}} c a_{i_{n}}=\lambda_{h} c a_{h}$. No passo (4), o fato de que $c a_{j}=0$ para todo $a_{j}$ em $\mathcal{D} \backslash\left\{a_{h}\right\}$ e que $c a_{h}=1$ garante que $\left(\mathcal{D} \backslash\left\{a_{h}\right\}\right) \cup\left\{a_{s}\right\}$ é linearmente independente e é diferente de $\mathcal{D}$.

Assim, podemos observar que a iteração está bem definida. Para demonstrar o teorema, resta mostrar que o algoritmo termina em número finito de iterações.

Seja $\mathcal{D}_{k}$ o conjunto $\mathcal{D}$ no início da $k$-ésima iteração. Vamos supor por contradição que o algoritmo não termina. Como o conjunto $\mathcal{D}$ muda no final de uma iteração e existe um número finito de escolhas para $\mathcal{D}$, podemos supor sem perda de generalidade que existe $l>k$ tal que $\mathcal{D}_{l}=\mathcal{D}_{k}$. Seja $r$ um índice tal que $a_{r}$ é removido de $\mathcal{D}$ no final de alguma iteração $p$, com $k \leq p<l$, e $r$ é o maior possível. Como $\mathcal{D}_{k}=\mathcal{D}_{l}$, existe uma iteração $q$, com $k \leq q<l$, na qual tal $a_{r}$ é adicionado a $\mathcal{D}_{q}$. Cabe observar que $q$ existe independentemente de $a_{r}$ pertencer a $\mathcal{D}_{k}$ ou não. 
Por definição, temos $\mathcal{D}_{p} \cap\left\{a_{r+1}, \ldots, a_{m}\right\}=\mathcal{D}_{q} \cap\left\{a_{r+1}, \ldots, a_{m}\right\}$. Vamos supor $\mathcal{D}_{p}=\left\{a_{i_{1}}, \ldots, a_{i_{n}}\right\}$ e $b=\lambda_{i_{1}} a_{i_{1}}+\cdots+\lambda_{i_{n}} a_{i_{n}}$. Se $c^{\prime}$ é o vetor $c$ obtido no passo (2) da iteração $q$, então temos por construção que $0>c^{\prime} b=\lambda_{i_{1}} c^{\prime} a_{i_{1}}+\cdots+\lambda_{i_{n}} c^{\prime} a_{i_{n}}$.

Vamos então analisar o valor de $\lambda_{i_{j}} c^{\prime} a_{i_{j}}$ para cada $i_{j}$ possível. Se $i_{j}<r$, então pelo passo (2) na iteração $p$ e pelo passo (4) na iteração $q$ sabemos respectivamente que $\lambda_{i_{j}} \geq 0$ e $c^{\prime} a_{i_{j}} \geq 0$. Pelo mesmo argumento, temos $\lambda_{r}<0$ e $c^{\prime} a_{r}<0$. Finalmente, se $i_{j}>r$, temos que $a_{i_{j}}$ está em $\mathcal{D}_{q}$, pois sabemos que $a_{i_{j}}$ está em $\mathcal{D}_{p}$ e $\mathcal{D}_{p} \cap\left\{a_{r+1}, \ldots, a_{m}\right\}=\mathcal{D}_{q} \cap\left\{a_{r+1}, \ldots, a_{m}\right\}$. Mas pela maximalidade de $r$, temos $a_{i_{j}} \neq a_{h}$ na iteração $q$ e concluímos que $c^{\prime} a_{i_{j}}=0$. Logo, temos a contradição $\lambda_{i_{1}} c^{\prime} a_{i_{1}}+\cdots+\lambda_{i_{n}} c^{\prime} a_{i_{n}} \geq 0$ e mostramos que o algoritmo termina.

Um corolário direto do teorema das desigualdades lineares é o conhecido lema de Farkas, que pode ser considerado como uma "versão não-negativa" do teorema fundamental da álgebra linear:

Corolário 2.2. Seja A uma matriz e seja $b$ um vetor. Existe um vetor não-negativo $x$ tal que $A x=b$ se e somente se não existe um vetor y tal que $y A \geq 0$ e $y b<0$.

Demonstração. Se existe $x$ tal que $x \geq 0$ e $A x=b$, então trivialmente temos que $y b=y A x \geq 0$ para todo $y \operatorname{com} y A \geq 0$. Suponha então que não existe $x \geq 0$ tal que $A x=b$ e sejam $a_{1}, \ldots, a_{m}$ as colunas de $A$. Segue direto do teorema 2.1 que existe um vetor $y$ tal que $y b<0$ e $y A \geq 0$.

Diversas formulações alternativas do lema de Farkas podem ser demonstradas. Podemos, por exemplo, caracterizar a existência de algum vetor $x$ tal que $A x \leq b$ ao invés de $A x=b$ e $x \geq 0$ :

Corolário 2.3. Seja $A$ uma matriz e seja $b$ um vetor. Existe um vetor $x$ tal que $A x \leq b$ se $e$ somente se não existe um vetor não-negativo y tal que yA=0 e yb<0.

Demonstração. Seja $A^{\prime}$ uma matriz tal que $A^{\prime}=[I A-A]$. Podemos verificar que o sistema $A x \leq b$ tem solução se e somente se $A^{\prime} x=b$ tem solução não-negativa. Pelo corolário 2.2 , isso é verdade se e somente se $y b \geq 0$ para todo $y \operatorname{com} y A^{\prime} \geq 0$, ou seja, $y \geq 0$ tal que $y A=0$ pela definição de $A^{\prime}$.

Um sistema da forma $A x \leq b$ é chamado de sistema de desigualdades lineares. Os conceitos de solução e resolver são análogos aos de sistemas de equações lineares. Se $a x \leq \beta$ é uma das desigualdades desse sistema, dizemos que $a x \leq \beta$ é uma restrição de $A x \leq b$.

A partir da matriz $A^{\prime}$ que foi usada na demonstração do corolário 2.3, segue naturalmente que:

Lema 2.4. Resolver um sistema de desigualdades lineares é polinomialmente equivalente a resolver um sistema de equaçôes lineares sob a restrição de que a solução deve ser não-negativa.

Na seção a seguir, vamos além da simples resolução de sistemas. Vamos considerar otimizações sobre sistemas de desigualdades lineares. Nisso consiste a área conhecida como programação linear.

\subsection{Programação linear e dualidade}

Um poliedro é o conjunto de todas as soluções de um sistema de desigualdades lineares. Mais formalmente, dizemos que dado $\mathcal{P}$ é um poliedro se existem uma matriz $A$ e um vetor $b$ tais que

$$
\mathcal{P}=\{x: A x \leq b\} .
$$

Resumidamente, um programa linear consiste no problema de otimizar uma dada função linear sobre um poliedro. Por exemplo, podemos maximizar $c x \operatorname{com} x$ restrito ao conjunto $\{x: A x \leq b\}$. 
Denotaremos esse programa por

$$
\max \{c x: A x \leq b\}
$$

Resolver um programa linear significa obter um vetor que pertence ao poliedro correspondente e otimiza a função dada. Esse vetor é chamado de solução do programa. Se um programa não admite solução, então seu poliedro é vazio ou não existe um limitante, nesse caso superior, para o valor da função dentro do poliedro. Se admite solução, o valor da função nas soluções é o valor ótimo.

O programa linear dual a $(2.2)$ é

$$
\min \{y b: y A=c ; y \geq 0\}
$$

Um dos resultados mais importantes em programação linear é o teorema da dualidade, que neste texto será apresentado como simples corolário do teorema fundamental das desigualdades:

Teorema 2.5. Seja A uma matriz e sejam b,c vetores. Se os programas lineares (2.2) e (2.3) admitem solução, então

$$
\max \{c x: A x \leq b\}=\min \{y b: y A=c ; y \geq 0\} .
$$

Demonstração. Se $A x \leq b, y \geq 0$ e $y A=c$, então $c x=y A x \leq y b$. Isso implica que

$$
\max \{c x: A x \leq b\} \leq \min \{y b: y \geq 0, y A=c\} .
$$

Assim, para obter o resultado desejado, é suficiente obter dois vetores, $x$ e $y$, que satisfazem $A x \leq b$, $y \geq 0, y A=c$ e $y b \leq c x$. Em termos de matrizes, queremos obter $x$ e $y$ satisfazendo

$$
\left[\begin{array}{cc}
A & 0 \\
-c & b \\
0 & A^{\prime} \\
0 & -A^{\prime}
\end{array}\right]\left(\begin{array}{l}
x \\
y
\end{array}\right) \leq\left(\begin{array}{c}
b \\
0 \\
c \\
-c
\end{array}\right)
$$

onde $A^{\prime}$ é a transposta de $A$. Aplicando o corolário 2.3, sabemos que tais $x$ e $y$ existem se e somente se $u b+v c-w c \geq 0$ para quaisquer $u, \lambda, v, w \geq 0$ que satisfazem as condições $u A-\lambda c=0$ e $\lambda b+A v-A w=0$. Vamos provar que isso é verdade.

Considere $u, \lambda, v, w$ satisfazendo as condições descritas. Se $\lambda>0$, então temos

$$
u b=\lambda^{-1} u \lambda b=\lambda^{-1} u A(w-v)=\lambda^{-1} \lambda c(w-v)=w c-v c .
$$

Se $\lambda=0$, considere $x_{0}, y_{0} \operatorname{com} A x_{0} \leq b, y_{0} \geq 0$ e $y_{0} A=c$. Nesse caso temos que

$$
u b \geq u A x_{0}=\lambda c x_{0}=0=y_{0} \lambda b=y_{0} A(w-v)=c(w-v)=w c-v c .
$$

Portanto, segue que $u b+v c-w c \geq 0$, o que conclui a demonstração.

A igualdade (2.4) é conhecida como equação de dualidade. Na verdade, para que essa equação seja válida, é suficiente demonstrar apenas que um de seus dois problemas admite solução, pois:

Teorema 2.6. Seja A uma matriz e sejam b,c vetores. O programa linear (2.2) admite solução se e somente se o programa linear (2.3) admite solução. 
Demonstração. Se não existe $x$ tal que $A x \leq b$, então pelo corolário 2.3 existe $y$ não-negativo tal que $y A=0$ e $y b<0$. Logo, se existe uma solução $y^{*}$ de $(2.3)$, então $y^{*}+\lambda y$ pertence ao poliedro desse programa para todo $\lambda>0$. Mas isso é uma contradição, pois $\left(y^{*}+\lambda y\right) b<y^{*} b$.

Se o valor de $c x$ não é limitado superiormente no poliedro de (2.2), então podemos verificar que existe algum $x$ tal que $A x \leq 0$ e $c x>0$ : basta tomar uma direção ao longo da qual o valor do custo pode crescer arbitrariamente. Logo, pelo corolário 2.2, o programa (2.3) não admite solução. A demonstração para a recíproca é completamente análoga e usa os mesmos corolários.

Logo, podemos concluir que a dualidade reduz problemas de otimização a problemas de existência:

Lema 2.7. Resolver um programa linear é polinomialmente equivalente a resolver um sistema de desigualdades lineares.

Demonstração. Pelo teorema 2.5, obter soluções para os programas lineares (2.2) e (2.3) equivale a obter $x, y$ tais que $x$ pertence ao poliedro de (2.2), $y$ pertence ao poliedro de $(2.3)$ e $c x=y b$.

Unindo os lemas 2.4 e 2.7, podemos concluir que os problemas que vimos até agora equivalem:

Teorema 2.8. Resolver um programa linear é polinomialmente equivalente a resolver um sistema de equações lineares sob a restrição de que a solução deve ser não-negativa.

A figura 2.1 ilustra como o teorema da dualidade pode ser interpretado de maneira geométrica. Se $\delta:=\max \{c x: A x \leq b\}$, então o conjunto $\{x: c x=\delta\}$ é obtido deslocando-se ao máximo possível, na direção do vetor $c$, um hiperplano perpendicular a $c$, sem deixar de intersectar $\{x: A x \leq b\}$.

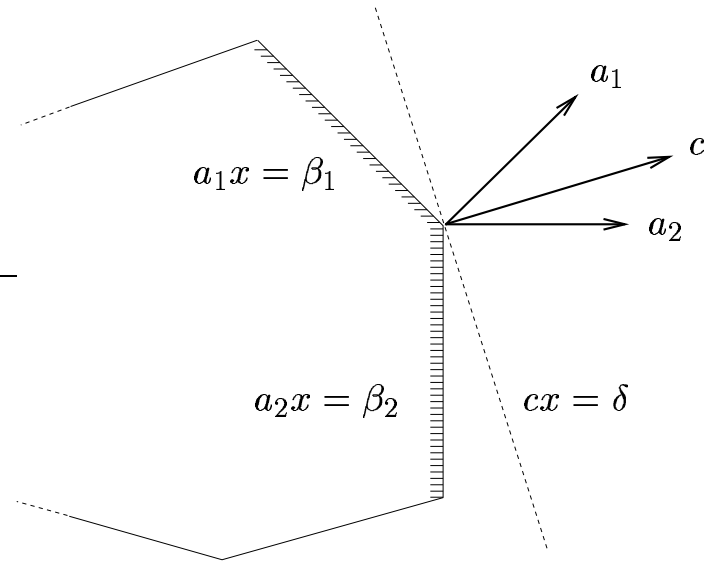

Figura 2.1: interpretação geométrica da dualidade.

Observando a representação gráfica, podemos verificar que $c x \leq \delta$ é uma combinação linear não-negativa das desigualdades $a_{1} x \leq \beta_{1}$ e $a_{2} x \leq \beta_{2}$ do sistema $A x \leq b$. Logo, existe $y \geq 0$ tal que $y A=c$ e $y b=\delta$. Isso implica que $\max \{c x: A x \leq b\} \geq \min \{y b: y \geq 0 ; y A=c\}$.

Note que essa combinação linear não-negativa que mencionamos usa apenas as restrições que são satisfeitas com igualdade por uma solução do problema de maximização. Esse fenômeno é conhecido como folgas complementares. Vamos formalizar melhor esse resultado no corolário a seguir:

Corolário 2.9. Seja A uma matriz e sejam $b, c, x^{*}, y^{*}$ vetores. Suponha que $x^{*}$ pertence ao poliedro do programa linear (2.2) e que $y^{*}$ pertence ao poliedro do programa linear (2.3). Os vetores $x^{*}, y^{*}$ são soluções desses respectivos programas se e somente se, para cada elemento positivo de $y^{*}$, a restrição de $A x \leq b$ correspondente a esse elemento é satisfeita com igualdade por $x^{*}$. 
Demonstração. Sabemos que $c x^{*}=y^{*} A x^{*} \leq y^{*} b$ e sabemos pelo teorema 2.5 que $x^{*}, y^{*}$ são soluções dos respectivos programas se e somente se $y^{*} A x^{*}=y^{*} b$. Mas isso equivale a $y^{*}\left(b-A x^{*}\right)=0$.

Informalmente, esse corolário afirma que para cada linha da matriz, há igualdade em pelo menos uma das duas soluções. Podemos demonstrar também que, para qualquer linha, é possível escolher esse par de soluções de tal forma que há igualdade em apenas uma delas. Primeiramente, vamos apresentar uma "versão afim" do lema de Farkas que facilitará a demonstração dessa afirmação:

Lema 2.10. Seja $A$ uma matriz e sejam b,c vetores. Suponha que o poliedro (2.1) é não-vazio. Se $c x \leq \delta$ para todo $x$ tal que $A x \leq b$, então existem $\delta^{\prime} \leq \delta$ e $y \geq 0$ tais que $y A=c$ e $y b=\delta^{\prime}$.

Demonstração. Primeiramente, vamos mostrar que o programa (2.3) admite solução. Se o poliedro desse programa é vazio, então pelo corolário 2.2 existe um vetor $z$ tal que $A z \leq 0$ e $c z>0$. Mas então, se $x$ é tal que $A x \leq b$, temos que $A(x+\lambda z)=A x+\lambda A z \leq b$ e $c(x+\lambda z)=c x+\lambda c z>c x$ para todo $\lambda>0$. Isso contradiz a hipótese de que $c x \leq \delta$ se $A x \leq b$.

Por outro lado, se não há limitante inferior para $y b$ em (2.3), segue pelo corolário 2.3 que não pode existir $x$ tal que $A x \leq 0$, outra contradição. Podemos então considerar a equação de dualidade (2.4). Sabendo que os dois poliedros dessa equação são não-vazios, a dualidade de fato é válida. Portanto, se tomarmos $\delta^{\prime}:=\max \{c x: A x \leq b\}$, o resultado desejado segue direto.

Com a ajuda desse lema, podemos demonstrar então uma extensão das folgas complementares:

Corolário 2.11. Seja A uma matriz, sejam $b, c$ vetores e seja ax $\leq \beta$ uma restrição de $A x \leq b$. Suponha que os programas lineares (2.2) e (2.3) admitem solução. Uma e somente e somente uma dentre as afirmações a seguir é verdadeira:

(i) existe uma solução $x^{*}$ de (2.2) tal que ax $x^{*}<\beta$;

(ii) existe uma solução $y^{*}$ de (2.3) cujo elemento correspondente a ax $\leq \beta$ é positivo.

Demonstração. As afirmações (i) e (ii) não são simultaneamente verdadeiras pelo corolário 2.9. Vamos então supor que (i) não vale, ou seja, não existe uma solução $x^{*}$ para $(2.2)$ com $a x^{*}<\beta$. Seja $\delta$ o valor ótimo. Então, $A x \leq b, c x \geq \delta$ implica $a x \geq \beta$.

Podemos concluir então que a desigualdade $-a x \leq-\beta$ é válida para todo vetor $x$ que satisfaz

$$
\left[\begin{array}{r}
A \\
-c
\end{array}\right] x \leq\left(\begin{array}{r}
b \\
-\delta
\end{array}\right)
$$

Pelo lema 2.10, existe $\delta^{\prime} \leq-\beta$ tal que $-a x \leq \delta^{\prime}$ é combinação não-negativa das desigualdades desse sistema. Ou seja, existem $y, \lambda \geq 0$ que satisfazem $y A-\lambda c=-a$ e $y b-\lambda \delta=\delta^{\prime} \leq-\beta$.

Seja $y^{\prime}$ o vetor obtido aumentando-se em 1 o elemento de $y$ correspondente a $a x \leq \beta$. Se $\lambda=0$, então $y A+a=0$ e $y b+\beta \leq 0$, ou seja, $y^{\prime} A=0$ e $y^{\prime} b \leq 0$. Logo, se $y^{*}$ é uma solução ótima para o mínimo, então $y^{*}+y^{\prime}$ também é. Como $y^{*}$ e $y$ são não-negativos, $y^{*}+y^{\prime}$ é a solução desejada para satisfazer (ii). Se $\lambda>0$, então $\lambda^{-1} y^{\prime}$ é solução ótima do mínimo: $\lambda^{-1} y^{\prime} A=\lambda^{-1}(y A+a)=\lambda^{-1} \lambda c=c$ e $\lambda^{-1} y^{\prime} b=\lambda^{-1}(y b+\beta) \leq \lambda^{-1} \lambda \delta=\delta$. Portanto, também temos a solução desejada para (ii).

Uma outra conseqüência dessas folgas é a identificação de uma solução a partir de outra solução:

Corolário 2.12. Seja A uma matriz, seja b um vetor, seja $y^{\prime}$ uma solução do programa linear (2.3) e seja $y^{\prime \prime}$ um vetor que pertence ao poliedro desse programa linear. Se um elemento de $y^{\prime \prime}$ é positivo somente se o elemento correspondente em $y^{\prime}$ é positivo, então $y^{\prime \prime}$ é uma solução de (2.3). 
Demonstração. Segue diretamente do corolário 2.9, pois tal $y^{\prime \prime}$ satisfaz folgas complementares.

Se um dado $x$ satisfaz $a x=\beta$, onde $a x \leq \beta$ é uma restrição de $A x \leq b$, então dizemos que essa restrição é ativa em $x$. Estendendo a definição, dizemos também que $a$ é uma linha ativa em $x$.

\subsection{Aspectos estruturais de poliedros}

Seja $a x \leq \beta$ uma restrição de $A x \leq b$. Se $a \neq 0$ e $a x=\beta$ para todo $x$ tal que $A x \leq b$, então essa restrição é uma igualdade implícita desse sistema. Senão, é uma restrição livre de igualdade. A partir desse ponto, vamos sempre supor que toda restrição satisfaz $a \neq 0$.

O subsistema de igualdades implícitas de $A x \leq b$ é o sistema formado pelas igualdades implícitas desse sistema. Analogamente, o subsistema livre de igualdades de $A x \leq b$ é o sistema formado por todas as restrições livres de igualdade desse sistema.

Dizemos que um poliedro tem dimensão plena se nenhuma das restrições do sistema que o define é igualdade implícita. Dizemos também que, se um vetor contém apenas um elemento não-nulo e esse elemento é 1, então esse vetor é canônico. A partir do conceito de vetores canônicos, podemos enunciar o teorema a seguir, que define um caso particular de poliedros que possuem dimensão plena:

Teorema 2.13. Se um poliedro contém todos os vetores canônicos e contém o vetor nulo, então esse poliedro tem dimensão plena.

Demonstração. Seja $a x \leq \beta$ uma restrição do sistema que define o poliedro e suponha que essa restrição é uma igualdade implícita. Como o vetor nulo pertence ao poliedro, sabemos que $\beta=0$. Mas isso é uma contradição, pois $a \neq 0$ e todos os vetores canônicos pertencem ao poliedro.

Um subconjunto não-vazio de um poliedro é uma face desse poliedro se for o conjunto de soluções de algum programa linear definido sobre ele. As faces podem ser caracterizadas pelo teorema dado a seguir. Cabe observar que, na demonstração desse teorema e em todo o restante desse texto, utilizaremos a expressão $x>0$ para um dado vetor $x$ quando todos os seus elementos forem positivos.

Teorema 2.14. Seja $A$ uma matriz, seja $b$ um vetor e seja $\mathcal{P}$ o poliedro (2.1). Um conjunto $\mathcal{F}$ é uma face de $\mathcal{P}$ se e somente se $\mathcal{F}$ é não-vazio e, para algum subsistema $A^{\prime} x \leq b^{\prime}$ de $A x \leq b$,

$$
\mathcal{F}=\left\{x \in \mathcal{P}: A^{\prime} x=b^{\prime}\right\}
$$

Demonstração. Seja $c$ um vetor tal que o programa linear (2.2) admite solução, seja $\delta$ o valor ótimo desse programa e seja $\mathcal{F}$ a face de $\mathcal{P}$ formada pelas soluções desse programa. Se $c$ é o vetor nulo, segue trivialmente que $\delta=0$ e $\mathcal{F}=\mathcal{P}$. Suponha então que esse vetor $c$ é não-nulo.

Pelo teorema 2.5, temos $\delta=\min \{y b: y \geq 0, y A=c\}$. Seja $y^{*}$ uma solução para esse problema de minimização e seja $A^{\prime} x \leq b^{\prime}$ o subsistema de $A x \leq b$ induzido pelas coordenadas positivas de $y^{*}$. Se $A x \leq b$, então $c x=\delta$ se e somente se $y^{*} A x=y^{*} b$, pois temos

$$
c x=y^{*} A x \leq y^{*} b=\delta .
$$

Ademais, $y^{*} A x=y^{*} b$ se e somente se $A^{\prime} x=b^{\prime}$ por folgas complementares.

Considere agora $\mathcal{F}=\left\{x \in P: A^{\prime} x=b^{\prime}\right\}$, onde $A^{\prime} x \leq b^{\prime}$ é um subsistema de $A x \leq b$, e suponha que $\mathcal{F}$ é não-vazio. Seja $y>0$ e $c:=y A$. Como $A^{\prime} x \leq b^{\prime}$ para todo $x \in \mathcal{P}$ e $\mathcal{F}$ é não-vazio, então $\mathcal{F}$ é o conjunto das soluções de $\max \{c x: x \in \mathcal{P}\}$ por folgas complementares. 
Dizemos que um subsistema $A^{\prime} x \leq b^{\prime}$ de $A x \leq b$ é minimal se $\left\{x: A^{\prime \prime} x \leq b^{\prime \prime}\right\} \subset\left\{x: A^{\prime} x \leq b^{\prime}\right\}$ para todo subsistema $A^{\prime \prime} x \leq b^{\prime \prime}$ de $A^{\prime} x \leq b^{\prime}$ com menos restrições do que o próprio $A^{\prime} x \leq b^{\prime}$. Dizemos também que restrições $a_{1} x \leq \beta_{1}$ e $a_{2} x \leq \beta_{2}$ são equivalentes se $\left\{x: a_{1} x \leq \beta_{1}\right\}=\left\{x: a_{2} x \leq \beta_{2}\right\}$.

Uma faceta de um poliedro é uma face maximal que não é o próprio poliedro. Temos então que:

Teorema 2.15. Seja $A$ uma matriz, seja $b$ um vetor, seja $\mathcal{P}$ o poliedro (2.1) e seja $A^{\prime} x \leq b^{\prime} o$ subsistema livre de igualdades de $A x \leq b$. Suponha que esse subsistema é minimal. Se $a^{\prime} x \leq \beta^{\prime}$ e $a^{\prime \prime} x \leq \beta^{\prime \prime}$ são restrições de $A^{\prime} x \leq b^{\prime}$, então existem facetas $\mathcal{F}^{\prime}$ e $\mathcal{F}^{\prime \prime}$ de $\mathcal{P}$ tais que

$$
\mathcal{F}^{\prime}=\left\{x \in \mathcal{P}: a^{\prime} x=\beta^{\prime}\right\} \quad e \quad \mathcal{F}^{\prime \prime}=\left\{x \in \mathcal{P}: a^{\prime \prime} x=\beta^{\prime \prime}\right\}
$$

Ademais, se essas restrições são não-equivalentes, então $\mathcal{F}$ e $\mathcal{F}^{\prime \prime}$ são facetas distintas.

Demonstração. Seja $A^{\prime \prime} x \leq b^{\prime \prime}$ o subsistema obtido eliminando-se $a^{\prime} x \leq \beta^{\prime}$ de $A^{\prime} x \leq b^{\prime}$. Vamos provar que existe $x^{*} \in \mathcal{P}$ tal que $A^{\prime \prime} x^{*}<b^{\prime \prime}$ e $a^{\prime} x^{*}=\beta^{\prime}$. Podemos verificar que existe $x^{\prime} \in \mathcal{P}$ tal que $A^{\prime} x^{\prime}<b^{\prime}$ : considere o baricentro dos vetores que satisfazem alguma restrição de maneira estrita. Como estamos supondo que $A^{\prime} x \leq b^{\prime}$ é minimal, então também existe $x^{\prime \prime} \in \mathcal{P}$ tal que $A^{\prime \prime} x^{\prime \prime} \leq b^{\prime \prime}$ e $a^{\prime} x^{\prime \prime}>\beta^{\prime}$. Podemos então tomar $x^{*}$ como uma combinação convexa de $x^{\prime}$ e $x^{\prime \prime}$. Portanto, as soluções de $\max \left\{x \in \mathcal{P}: a^{\prime} x=\beta^{\prime}\right\}$ são uma faceta.

A demonstração para $a^{\prime \prime} x \leq \beta^{\prime \prime}$ é análoga. Vamos agora supor que as duas restrições são não-equivalentes. Em particular, podemos supor que existe $z$ tal que $a^{\prime} z>\beta^{\prime}$ e $a^{\prime \prime} z \leq \beta^{\prime \prime}$. Podemos supor sem perda de generalidade que $A^{\prime \prime} z \leq b^{\prime \prime}$, pois se isso não for verdade basta substituir $z$ por uma combinação convexa adequada de $z$ e $x^{*}$. Por fim, tomando uma combinação convexa adequada de $z$ e $x^{\prime}$, podemos obter um vetor em $\mathcal{P}$ que pertence a $\mathcal{F}^{\prime}$ mas não pode pertencer a $\mathcal{F}^{\prime \prime}$.

Uma recíproca desse teorema também pode ser demonstrada como mostraremos a seguir:

Teorema 2.16. Seja $A$ uma matriz, seja $b$ um vetor, seja $\mathcal{P}$ o poliedro (2.1) e seja $A^{\prime} x \leq b^{\prime}$ o subsistema livre de igualdades de $A x \leq b$. Suponha que esse subsistema é minimal. Se $\mathcal{F}^{\prime}$ e $\mathcal{F}^{\prime \prime}$ são facetas de $\mathcal{P}$, então existem restrições $a^{\prime} x \leq \beta^{\prime}$ e $a^{\prime \prime} x \leq \beta^{\prime \prime}$ de $A^{\prime} x \leq b^{\prime}$ tais que

$$
\mathcal{F}^{\prime}=\left\{x \in \mathcal{P}: a^{\prime} x=\beta^{\prime}\right\} \quad e \quad \mathcal{F}^{\prime \prime}=\left\{x \in \mathcal{P}: a^{\prime \prime} x=\beta^{\prime \prime}\right\}
$$

Ademais, se essas facetas são distintas, então $a^{\prime} x \leq b^{\prime}$ e $a^{\prime \prime} x \leq b^{\prime \prime}$ são restrições não-equivalentes.

Demonstração. Seja $A^{\prime \prime} x \leq b^{\prime \prime}$ um subsistema de $A^{\prime} x \leq b^{\prime}$ tal que $\mathcal{F}^{\prime}=\left\{x \in \mathcal{P}: A^{\prime \prime} x=b^{\prime \prime}\right\}$. Seja $a^{\prime} x \leq \beta^{\prime}$ uma desigualdade desse subsistema. Como $\left\{x \in \mathcal{P}: a^{\prime} x=\beta^{\prime}\right\}$ é não-vazio, esse conjunto é uma face de $\mathcal{P}$ que contém $\mathcal{F}$. Ademais, esse conjunto não é igual a $\mathcal{P}$, pois $a^{\prime} x \leq \beta^{\prime}$ não e uma igualdade implícita. Logo, $\mathcal{F}^{\prime}=\left\{x \in \mathcal{P}: a^{\prime} x=\beta^{\prime}\right\}$ por maximalidade.

A demonstração para $\mathcal{F}^{\prime \prime}$ é análoga. Vamos agora supor que as facetas são distintas. Nesse caso, existe $x^{\prime}$ tal que $a^{\prime} x^{\prime}=\beta^{\prime}$ e $A^{\prime \prime} x^{\prime}<b^{\prime \prime}$, onde $A^{\prime \prime} x \leq b^{\prime \prime}$ é o subsistema obtido eliminando-se $a^{\prime} x \leq \beta^{\prime}$ de $A^{\prime} x \leq b^{\prime}$. Segue então que as restrições $a^{\prime} x \leq \beta^{\prime}$ e $a^{\prime \prime} x \leq \beta^{\prime \prime}$ não podem ser equivalentes.

Esses dois teoremas caracterizam a correspondência biunívoca entre restrições e facetas. Uma conseqüência direta dessa correspondência é o seguinte resultado para poliedros de dimensão plena:

Corolário 2.17. Sejam $A, C$ matrizes, sejam $b, d$ vetores e sejam

$$
\mathcal{P}:=\{x: A x \leq b\} \quad \text { e } \quad \mathcal{Q}:=\{x: C x \leq d\} .
$$


Seja ax $\leq \beta$ uma restrição de $A x \leq b$, seja $c x \leq \delta$ uma restrição de $C x \leq d$ e sejam

$$
\mathcal{F}:=\{x \in \mathcal{P}: a x=\beta\} \quad \text { e } \quad \mathcal{G}:=\{x \in \mathcal{Q}: c x=\delta\} .
$$

Suponha que $\mathcal{P}$ tem dimensão plena, que $A x \leq b$ é minimal e que $\mathcal{P}$ é um subconjunto de $\mathcal{Q}$. Se $\mathcal{F}$ é um subconjunto de $\mathcal{G}$, então ax $\leq \beta$ e $c x \leq \delta$ são equivalentes.

Demonstração. Podemos verificar pela correspondência biunívoca entre faces e restrições que $\mathcal{F}$ e $\mathcal{G}$ são facetas de $\mathcal{P}$. Se supusermos que $a x \leq \beta$ e $c x \leq \delta$ são não-equivalentes, podemos chegar a uma contradição através de argumentos de combinação convexa similares aos que já vimos antes.

Esse corolário nos permite provar a unicidade do sistema minimal que determina um poliedro:

Corolário 2.18. Se um poliedro tem dimensão plena, então existe um único sistema minimal que determina esse poliedro, a menos da substituição de uma restrição por uma restrição equivalente.

Demonstração. Segue do corolário 2.17 quando aplicado a sistemas definindo o mesmo poliedro.

Por fim, vamos considerar faces minimais de um poliedro. Por resultados anteriores, temos que:

Teorema 2.19. Seja $A$ uma matriz e seja $b$ um vetor. Um conjunto $\mathcal{F}$ é uma face minimal do poliedro (2.1) se e somente se $\emptyset \subset \mathcal{F} \subseteq \mathcal{P}$ e, para algum subsistema $A^{\prime} x \leq b^{\prime}$ de $A x \leq b$,

$$
\mathcal{F}=\left\{x: A^{\prime} x=b^{\prime}\right\}
$$

Demonstração. Seja $\mathcal{F}=\left\{x: A^{\prime} x=b^{\prime}\right\}$. Se $\emptyset \subset \mathcal{F} \subseteq \mathcal{P}$, então $\mathcal{F}=\left\{x \in \mathcal{P}: A^{\prime} x=b^{\prime}\right\}$ e portanto é uma face do poliedro. Como a correspondência biunívoca garante que $\left\{x: A^{\prime} x=b^{\prime}\right\}$ é um poliedro sem facetas, podemos concluir que $\mathcal{F}$ é uma face minimal.

Suponha agora que $\mathcal{F}$ é face minimal de $\mathcal{P}$. Podemos definir que $\mathcal{F}=\left\{x: A^{\prime \prime} x \leq b^{\prime \prime}, A^{\prime} x=b^{\prime}\right\}$, onde $A^{\prime \prime} x \leq b^{\prime \prime}$ e $A^{\prime} x \leq b^{\prime}$ são subsistemas de $A x \leq b$. Sem perda de generalidade, podemos supor que $A^{\prime \prime} x \leq b^{\prime \prime}$ é minimal. Por minimalidade, $\mathcal{F}$ é um poliedro que não pode ter facetas. Logo, pela correspondência biunívoca, podemos concluir que não há nenhuma restrição em $A^{\prime \prime} x \leq b^{\prime \prime}$.

Os dois corolários interessantes a seguir seguem diretamente da caracterização de faces minimais. Cabe observar que o termo poliedro limitado refere-se à definição topológica usual de limitação.

Corolário 2.20. Seja $\mathcal{P}$ um poliedro limitado. Se um vetor $x$ pertence a $\mathcal{P}$, então $x$ pertence ao fecho convexo de vetores que pertencem a faces minimais de $\mathcal{P}$.

Corolário 2.21. Seja $A$ uma matriz e seja $b$ um vetor. Suponha que Ax=0 se e somente se $x=0$. Se um conjunto $\mathcal{F}$ é uma face minimal do poliedro (2.1), então $\mathcal{F}$ contém somente um vetor.

Quando as faces minimais contêm somente um vetor, esse vetor é chamado de vértice do poliedro.

\subsection{Cones e o teorema de Carathéodory}

Sejam $a_{1}, \ldots, a_{m}$ vetores. O cone gerado por tais vetores é

$$
\operatorname{cone}\left(a_{1}, \ldots, a_{m}\right):=\left\{a: a=\lambda_{1} a_{1}+\cdots+\lambda_{m} a_{m}, \text { onde } \lambda_{1}, \ldots, \lambda_{m} \in \mathbb{R}_{\geq}\right\} .
$$

Mais informalmente, é o conjunto de todos os vetores gerados por combinações lineares não-negativas de $a_{1}, \ldots, a_{m}$. O lema de Farkas pode ser reformulado em termos de cones da seguinte maneira: 
Teorema 2.22. Sejam $a_{1}, \ldots, a_{m}, b$ vetores. $O$ vetor $b$ pertence ao cone de $a_{1}, \ldots, a_{m}$ se e somente se não existe um vetor y tal que yb<0 e ya $a_{i} \geq 0$ para todo $i$.

Essa formulação é uma "versão não-negativa" do teorema 1.11. Ademais, como KhaCHIYAN [1979] e KARMARKAR [1984] mostraram que um programa linear pode ser resolvido em tempo polinomial, segue do teorema 2.8 que podemos também obter uma "versão não-negativa" do teorema 1.12:

Teorema 2.23. Existe um algoritmo polinomial que, dados vetores $a_{1}, \ldots, a_{m}, b$, verifica se o vetor $b$ pertence ao cone de $a_{1}, \ldots, a_{m}$, devolve uma combinação linear não-negativa correspondente em caso positivo e devolve um vetor y tal que $y b<0$ e ya $a_{i} \geq 0$ para todo $i$ em caso negativo.

Vamos encerrar este capítulo com algumas propriedades importantes de cones. Primeiramente, vamos demonstrar que todo cone é um poliedro pelo teorema fundamental das desigualdades (2.1).

Teorema 2.24. Seja $\mathcal{C}$ um conjunto de vetores. Existe um conjunto finito $\mathcal{A}$ tal que $\mathcal{C}=\operatorname{cone}(\mathcal{A})$ se e somente se existe uma matriz $A$ tal que $\mathcal{C}=\{x: A x \leq 0\}$.

Demonstração. Considere um conjunto finito de vetores $\mathcal{A}=\left\{a_{1}, \ldots, a_{m}\right\}$. Vamos demonstrar que existe uma matriz $A$ tal que $\operatorname{cone}(\mathcal{A})=\{x: A x \leq 0\}$. Primeiramente, vamos demonstrar que podemos supor sem perda de generalidade que os vetores $a_{1}, \ldots, a_{m}$ geram todo o espaço. Se isso não for verdade, existe uma matriz não-singular $P$ tal que

$$
P C=\left[\begin{array}{c}
C^{\prime} \\
0
\end{array}\right]
$$

onde $C$ é uma matriz cujas colunas são $a_{1}, \ldots, a_{m}$ e $C^{\prime}$ é uma matriz com posto linha completo. As colunas de $C^{\prime}$, portanto, geram todo o espaço. Suponha então que existe uma matriz $A^{\prime}$ tal que $\left\{x: A^{\prime} x \leq 0\right\}$ é o cone gerado pelas colunas de $C^{\prime}$. Nesse caso, sabemos que $\{x: A x \leq 0\}$, onde

$$
A:=\left[\begin{array}{rr}
A^{\prime} & 0 \\
0 & I \\
0 & -I
\end{array}\right],
$$

é o cone gerado pelas colunas da matriz dada por $P C$. Podemos concluir então que $\{x: A P x \leq 0\}$ é o cone gerado pelas colunas de $C$, precisamente o conjunto cone $\left(a_{1}, \ldots, a_{m}\right)$. Portanto, podemos supor sem perda de generalidade que $a_{1}, \ldots, a_{m}$ geram todo o espaço.

Seja $r:=\operatorname{dim}(\mathcal{A})$ e seja $\mathcal{S}$ um conjunto de semi-espaços tal que $\{x: c x \leq 0\}$ pertence a $\mathcal{S}$ se e somente se $c a_{i} \leq 0$ para todo $i$ e pelo menos $r-1$ vetores linearmente independentes dentre $a_{1}, \ldots, a_{m}$ pertencem ao hiperplano $\{x: c x=0\}$. Pela segunda condição e pelo fato de que $a_{1}, \ldots, a_{m}$ geram todo o espaço, sabemos que a cardinalidade de $\mathcal{S}$ é finita.

Um vetor $b$ pertence a cone $(\mathcal{A})$ se e somente se $b=\lambda_{1} a_{1}+\cdots+\lambda_{m} a_{m}$, onde $\lambda_{1}, \ldots, \lambda_{m} \geq 0$. Sabemos que essa combinação existe se somente se o caso (ii) do teorema fundamental (2.1) não ocorre. Por outro lado, tal caso não ocorre se e somente se todo hiperplano $\{x: c x=0\}$ contendo pelo menos $r-1$ vetores linearmente independentes dentre $a_{1}, \ldots, a_{m}$ satisfaz $c b \leq 0$ se $c a_{i} \leq 0$ para todo $i$. Podemos concluir que $b$ pertence a $\operatorname{cone}(\mathcal{A})$ se e somente se pertence a todos os semi-espaços em $\mathcal{S}$. Portanto, se $A$ é uma matriz tal que $c$ é uma linha de $A$ se e somente se $\{x: c x \leq 0\} \in \mathcal{S}$, segue que cone $(\mathcal{A})=\{x: A x \leq 0\}$ e um dos lados dessa demonstração está concluído.

Considere agora uma matriz $A$ e sejam $c_{1}, \ldots, c_{n}$ as linhas dessa matriz. Como acabamos de demonstrar, sabemos que existe uma matriz $C$ tal que cone $\left(c_{1}, \ldots, c_{n}\right)=\{x: C x \leq 0\}$. Vamos demonstrar a seguir que, se $a_{1}, \ldots, a_{m}$ são as linhas de $C$, então $\{x: A x \leq 0\}=\operatorname{cone}\left(a_{1}, \ldots, a_{m}\right)$. 
Primeiramente, seja $a_{i}$ uma linha qualquer de $C$. Como qualquer vetor em cone $\left(c_{1}, \ldots, c_{n}\right)$ pertence a $\{x: C x \leq 0\}$, sabemos que $a_{i} c_{j} \leq 0$ para todo $j$. Portanto, $c_{j} a_{i} \leq 0$ para quaisquer $i, j$ e podemos assim concluir que cone $\left(a_{1}, \ldots, a_{m}\right) \subseteq\{x: A x \leq 0\}$.

Considere agora um vetor $b$ em $\{x: A x \leq 0\}$ e suponha por contradição que $b$ não pertence a cone $\left(a_{1}, \ldots, a_{m}\right)$. Novamente pelo teorema fundamental (2.1), sabemos que existe $c$ tal que $c a_{i} \leq 0$ para todo $i$ e $c b>0$. Temos então que $c \in\{x: C x \leq 0\}=\operatorname{cone}\left(c_{1}, \ldots, c_{n}\right)$ e, portanto, que existem $\lambda_{1}, \ldots, \lambda_{n} \geq 0$ tais que $c=\lambda_{1} c_{1}+\cdots+\lambda_{n} c_{n}$. Mas como $c_{1}, \ldots, c_{n}$ são as linhas de $A$, segue por hipótese que $c_{i} b \leq 0$ para todo $i$. Portanto, $c b=\lambda_{1} c_{1} b+\cdots+\lambda_{n} c_{n} b \leq 0$, uma contradição.

Note que, para toda matriz $A$, o conjunto $\{x: A x=0\}$ não é vazio: ele contém pelo menos o vetor nulo. Aplicando essa propriedade sobre o teorema anterior e os resultados anteriores a respeito de faces, podemos concluir que todo cone é um poliedro que contém uma única face minimal. Outra consequiência interessante do teorema anterior é o fato de que os fechos convexos são poliedros.

Corolário 2.25. O fecho convexo de um conjunto finito de vetores é um poliedro.

Demonstração. Segue diretamente do teorema 2.24 e do fato de que um vetor a pertence ao fecho convexo gerado por $a_{1}, \ldots, a_{m}$ se e somente se $(a 1)$ está no cone gerado por $\left(a_{1} 1\right), \ldots,\left(a_{m} 1\right)$.

Dizemos que um cone $\mathcal{C}$ é pontudo se não existe $c$ em $\mathcal{C}$ tal que $-c$ também está em $\mathcal{C}$. Temos:

Teorema 2.26. Sejam $a_{1}, \ldots, a_{m}$ vetores e seja $c$ um vetor que pertence ao cone gerado por esses vetores. Suponha que A é uma matriz tal que as colunas de $A$ são $a_{1}, \ldots, a_{m}$. Se o cone gerado por esses vetores é pontudo, então o programa linear $\max \{c x: A x=b ; x \geq 0\}$ admite solução.

Demonstração. Como $c$ pertence ao cone gerado por $a_{1}, \ldots, a_{m}$, o poliedro $\{x: A x=b ; x \geq 0\}$ é não-vazio. Portanto, o programa linear $\max \{c x: A x=b ; x \geq 0\}$ não admite solução somente se existe $x \geq 0$ tal que $A x=0$ e $c x>0$. Mas isso implica que tal $x$ é não-nulo e, portanto, que existe algum índice $i$ tal que $-a_{i}$ é combinação não-negativa das colunas de $A$. Isso é uma contradição.

Por fim, há o teorema de Carathéodory, outro corolário direto do teorema fundamental (2.1):

Teorema 2.27. Seja $\mathcal{A}$ um conjunto de vetores. Se b pertence ao cone de $\mathcal{A}$, então b é gerado por uma combinação não-negativa de vetores linearmente independentes em $\mathcal{A}$.

Demonstração. Como b pertence ao cone, a afirmação (i) do teorema 2.1 precisa ser verdadeira.

Esse teorema pode ser formulado também sobre um programa linear na forma dada por (2.3).

Teorema 2.28. Seja A uma matriz e sejam b,c vetores. Se o programa linear (2.3) admite solução, então existe uma solução y* para esse programa linear tal que as linhas de A correspondentes aos elementos não-nulos de $y^{*}$ são linearmente independentes.

Demonstração. Segue diretamente do teorema 2.27 e do corolário 2.12 aplicado sobre cada face.

Alternativamente, o teorema de Carathéodory pode ser formulado em termos de cardinalidade:

Corolário 2.29. Seja $\mathcal{A}$ um conjunto de vetores. Se b pertence ao cone de $\mathcal{A}$, então b é gerado por uma combinação não-negativa de não mais do que $\operatorname{dim}(\mathcal{A})$ vetores em $\mathcal{A}$.

Corolário 2.30. Seja A uma matriz e sejam b,c vetores. Se o programa linear (2.3) admite solução, então existe uma solução y* para esse programa linear tal que não mais do que posto $(A)$ elementos de $y^{*}$ são não-nulos.

Essa formulação em termos de cardinalidade será particularmente importante no capítulo 6 . 



\section{Capítulo 3}

\section{Sistemas diofantinos e reticulados}

Neste capítulo, vamos apresentar o problema de obter uma solução inteira para um sistema linear e mostrar que esse problema pode ser resolvido através de uma extensão natural do algoritmo de Euclides para determinar o máximo divisor comum de dois inteiros. Também mostraremos como o mesmo problema pode ser interpretado sob o ponto de vista de reticulados.

Enquanto no capítulo 2 estiveram presentes algumas "versões não-negativas" de resultados do capítulo 1, o objetivo deste capítulo é exibir algumas "versões inteiras" desses mesmos resultados.

\subsection{Algoritmo de Euclides}

Considere o seguinte problema: dados dois números inteiros, $\alpha_{1}$ e $\alpha_{2}$, queremos verificar se eles admitem máximo divisor comum e determiná-lo em caso positivo. Sabemos que esses inteiros admitem máximo divisor comum se e somente se $\alpha_{1} \neq 0$ ou $\alpha_{2} \neq 0$. Portanto, o problema pode ser reduzido a uma versão mais simples: dados dois inteiros tais que pelo menos um deles é não-nulo, calcular o máximo divisor comum. Vamos apresentar um algoritmo para efetuar esse cálculo.

Estamos supondo que $\alpha_{1} \neq 0$ ou $\alpha_{2} \neq 0$. Vamos estender essa estratégia de impor restrições sobre $\alpha_{1}$ e $\alpha_{2}$ sem perda de generalidade. Sabemos que, se um inteiro $\alpha$ é não-nulo, então seu maior divisor é $|\alpha|$. Logo, podemos verificar que $\operatorname{mdc}\left(\alpha_{1}, \alpha_{2}\right)=\operatorname{mdc}\left(\left|\alpha_{1}\right|,\left|\alpha_{2}\right|\right)$. Assim, com um simples procedimento para mudar o sinal de $\alpha_{1}$ ou $\alpha_{2}$ se necessário, podemos supor sem perda de generalidade que eles são ambos inteiros não-negativos.

Vamos agora considerar o caso em que um desses dois inteiros é zero. Suponha, por exemplo, que $\alpha_{1}>0$ e $\alpha_{2}=0$. Sabemos que o maior divisor de $\alpha_{1}$ é $\left|\alpha_{1}\right|$. Portanto, $\operatorname{mdc}\left(\alpha_{1}, \alpha_{2}\right)=\alpha_{1}$. Temos então um algoritmo para resolver esse caso particular do problema: para obter o máximo divisor comum, basta verificar quais dos dois inteiros é não-nulo. Para que esse caso nos seja útil, precisamos descrever um procedimento que nos permita supor sem perda de generalidade que ele ocorre.

Como nosso objetivo é transformar um dos dois dados inteiros não-negativos em zero, podemos considerar uma operação que os reduz. Cabe observar, no entanto, que tal operação deve preservar a não-negatividade, pois caso contrário não temos garantia de que os novos valores estão mais próximos de zero do que os valores originais. Uma idéia natural, portanto, é subtrair o menor inteiro do maior. Por exemplo, se $\alpha_{1}, \alpha_{2}>0$ e $\alpha_{2}$ é maior ou igual a $\alpha_{1}$, podemos verificar que:

$$
0 \leq \alpha_{2}-\alpha_{1}<\alpha_{2}
$$


Mais importante, porém, é verificar se $\operatorname{mdc}\left(\alpha_{1}, \alpha_{2}\right)=\operatorname{mdc}\left(\alpha_{1}, \alpha_{2}-\alpha_{1}\right)$. Esse invariante segue diretamente de um caso particular do seguinte resultado:

Lema 3.1. Se $\alpha_{1}, \alpha_{2}, \mu, \delta$ são inteiros, $\delta$ divide $\alpha_{1}$ e $\alpha_{2}$ se e somente se $\delta$ divide $\alpha_{1}$ e $\alpha_{2}+\mu \alpha_{1}$.

Demonstração. Se $\delta$ divide $\alpha_{1}$, então $\delta$ divide $\mu \alpha_{1}$, pois $\mu$ é inteiro. Logo, se $\delta$ divide $\alpha_{1}$ e $\alpha_{2}$, então $\delta$ divide $\alpha_{2}+\mu \alpha_{1}$. Analogamente, se $\delta$ divide $\alpha_{1}$ e $\alpha_{2}+\mu \alpha_{1}$, então $\delta$ divide $\left(\alpha_{2}+\mu \alpha_{1}\right)-\mu \alpha_{1}$.

A partir dessa redução, podemos agora descrever o algoritmo completo em pseudo-código, que recebe dois inteiros $\alpha_{1}, \alpha_{2}$ e devolve $\operatorname{mdc}\left(\alpha_{1}, \alpha_{2}\right)$ :

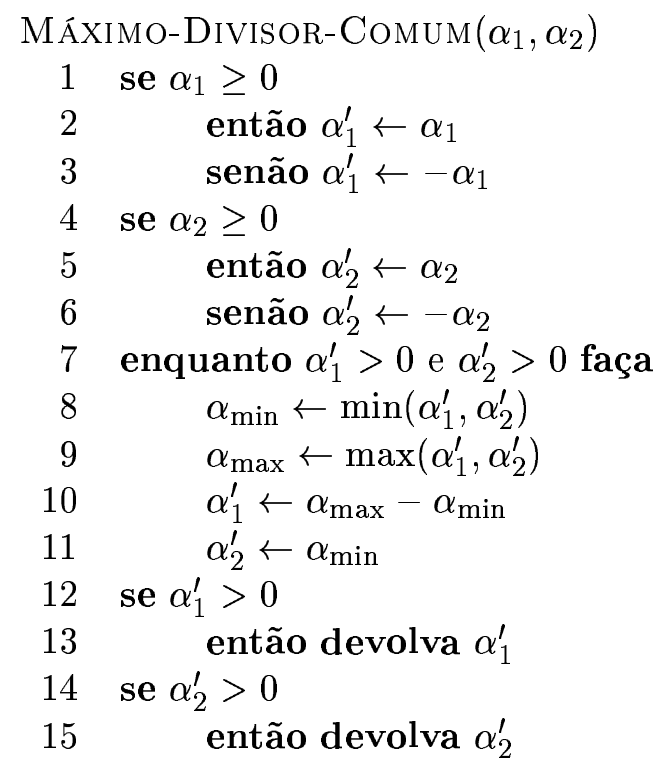

A partir da condição definida pela linha 5 e da redução efetuada pela linha 8 , podemos deduzir que o número de iterações do algoritmo é limitado por $\left|\alpha_{1}\right|+\left|\alpha_{2}\right|$. No entanto, tal limitante não é satisfatório, pois como o tamanho de $\alpha_{1}$ e $\alpha_{2}$ é dado a partir de seus logaritmos, temos que $\left|\alpha_{1}\right|+\left|\alpha_{2}\right|$ cresce exponencialmente com o tamanho da entrada.

Esse excesso de iterações executadas ao longo do algoritmo é conseqüência da possível necessidade de muitas subtrações. Para eliminar tal necessidade, uma idéia natural é subtrair o menor inteiro do maior o máximo possível de vezes em uma única iteração. Mais especificamente, o lema 3.1 nos permite considerar, ao invés de $\alpha_{\max }-\alpha_{\min }$, uma subtração mais eficiente dada por $\alpha_{\max }-\mu \alpha_{\min }$. Queremos que $\mu$ seja o maior inteiro satisfazendo $\alpha_{\max }-\mu \alpha_{\min } \geq 0$, condição que equivale a

$$
\mu \leq \frac{\alpha_{\max }}{\alpha_{\min }}
$$

A melhor opção para $\mu$, portanto, é dada por $\left\lfloor\alpha_{\max } / \alpha_{\min }\right\rfloor$. Podemos então descrever uma versão mais eficiente para calcular o máximo divisor comum, que foi proposta originalmente por Euclides: 


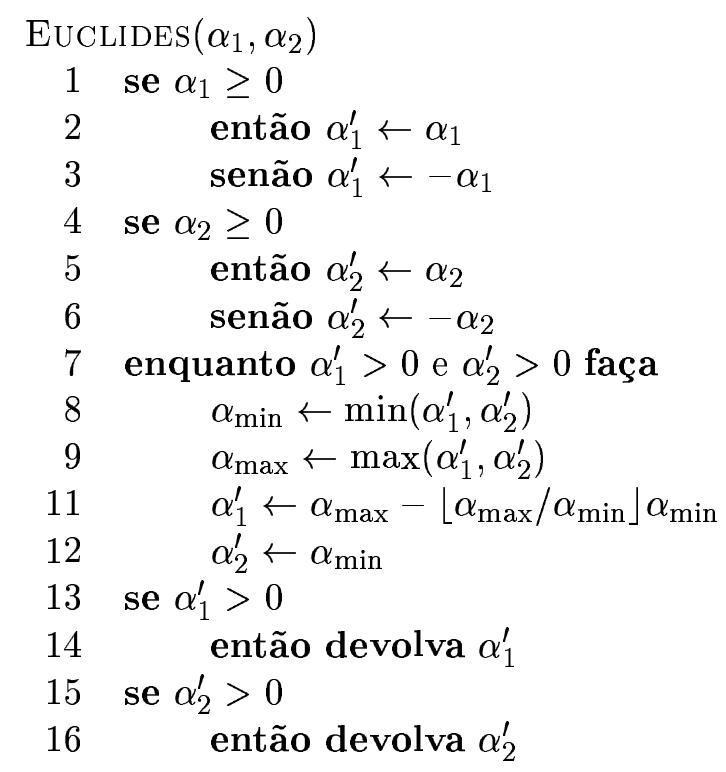

A eficiência dessa nova versão segue do fato de que cada iteração do algoritmo reduz ao menos pela metade o valor de um dos números, como demonstra o lema apresentado a seguir.

Lema 3.2. Se $\alpha_{1}$ e $\alpha_{2}$ são inteiros positivos e $\alpha_{2} \geq \alpha_{1}$, então $\alpha_{2}-\left\lfloor\alpha_{2} / \alpha_{1}\right\rfloor \alpha_{1} \leq \alpha_{2} / 2$.

Demonstração. Suponha por contradição que $\alpha_{2}-\left\lfloor\alpha_{2} / \alpha_{1}\right\rfloor \alpha_{1}>\alpha_{2} / 2$. Sob essa hipótese, temos que $\alpha_{2} / 2>\left\lfloor\alpha_{2} / \alpha_{1}\right\rfloor \alpha_{1}$. Segue, portanto, que $2\left\lfloor\alpha_{2} / \alpha_{1}\right\rfloor<\alpha_{2} / \alpha_{1}$, mas isso é uma contradição.

Assim, podemos concluir que o número de iterações executadas ao longo do algoritmo de Euclides é limitado superiormente por $\log \left|\alpha_{1}\right|+\log \left|\alpha_{2}\right|$, um valor polinomialmente limitado pelo tamanho de $\alpha_{1}$ e $\alpha_{2}$. Como as operações executadas são operações aritméticas simples, segue o teorema abaixo.

Teorema 3.3. Existe um algoritmo polinomial que, dois dados inteiros, verifica se eles admitem máximo divisor comum e o devolve em caso positivo.

O algoritmo de Euclides, no entanto, serve também para outros propósitos que veremos a seguir.

\subsection{Equações lineares diofantinas}

Vimos anteriormente o problema de resolver uma equação linear. Vamos agora considerar uma versão mais restrita desse problema: suponha que, dados $\alpha_{1}, \ldots, \alpha_{m}, \beta$, queremos obter $\xi_{1}, \ldots, \xi_{m}$ que satisfaçam

$$
\alpha_{1} \xi_{1}+\cdots+\alpha_{m} \xi_{m}=\beta \quad \text { e } \quad \xi_{1}, \ldots, \xi_{m} \in \mathbb{Z} .
$$

Uma restrição na forma acima é chamada de equação linear diofantina. Uma solução para a equação linear diofantina (3.1) é chamada de solução inteira para a equação linear

$$
\alpha_{1} \xi_{1}+\cdots+\alpha_{m} \xi_{m}=\beta \text {. }
$$

Vamos considerar o problema de verificar se uma equação linear admite solução inteira. Um caso simples em que a resposta é negativa é o caso em que a equação não admite nenhuma solução. 
Como vimos anteriormente, (3.2) não admite solução se e somente se $\beta \neq 0$ e $\alpha_{i}=0$ para todo $i$. Por outro lado, se $\beta=0$ e $\alpha_{i}=0$ para todo $i$, sabemos que quaisquer $\xi_{1}, \ldots, \xi_{m}$ satisfazem essa equação. Em particular, quaisquer $\xi_{1}, \ldots, \xi_{m}$ inteiros são soluções inteiras de (3.2).

Portanto, como esses dois casos podem ser resolvidos em tempo polinomial, vamos a partir de agora considerar apenas o caso em que $\alpha_{k} \neq 0$ para algum $k$. Como vimos anteriormente, temos nesse caso que a equação linear admite solução e ela pode ser obtida através de uma construção muito simples. No entanto, a existência de solução não garante a existência de solução inteira. Um exemplo simples disso é o caso em que todo $\alpha_{i}$ é um inteiro par e $\beta$ é um inteiro ímpar. Embora o problema admita solução nesse caso, ele não admite solução inteira.

Vamos então estender ainda mais a estratégia de restringir o problema, de forma a tornar o processo de verificação da existência de solução inteira suficientemente simples. Primeiramente, podemos observar que $\xi_{1}, \ldots, \xi_{m}$ satisfazem (3.2) se e somente se

$$
\delta \alpha_{1} \xi_{1}+\cdots+\delta \alpha_{m} \xi_{m}=\delta \beta
$$

para todo $\delta$. Em particular, para $\delta$ tal que $\delta \alpha_{1}, \ldots, \delta \alpha_{m}, \delta \beta$ são inteiros. Podemos, portanto, supor que $\alpha_{1}, \ldots, \alpha_{m}, \beta$ são inteiros, pois $\delta$ satisfazendo tal condição pode ser calculado em tempo polinomial. Vamos a seguir considerar o sinal de $\alpha_{1}, \ldots, \alpha_{m}$.

Podemos observar que $\xi_{1}, \ldots, \xi_{m}$ satisfazem (3.2) se e somente se

$$
\left(-\alpha_{1}\right)\left(-\xi_{1}\right)+\alpha_{2} \xi_{2}+\cdots+\alpha_{m} \xi_{m}=\beta .
$$

Logo, um algoritmo para verificar se existe solução inteira para a equação pode primeiramente trocar o sinal de todo $\alpha_{i}$ negativo. Cabe observar que, como estamos supondo que $\alpha_{k} \neq 0$ para algum $k$, estamos em uma situação análoga à do algoritmo de Euclides. De fato, se existe um único $k$ tal que $\alpha_{k} \neq 0$, temos um procedimento para resolver o problema, pois nesse caso existe solução inteira para a dada equação linear se e somente se existe $\xi_{k}$ inteiro tal que $\alpha_{k} \xi_{k}=\beta$.

Por outro lado, $\alpha_{k} \xi_{k}=\beta$ se e somente se $\xi_{k}=\alpha_{k}^{-1} \beta$. Logo, a verificação nesse caso se reduz a

$$
\text { existe solução inteira se e somente se } \alpha_{k}^{-1} \beta \text { é inteiro. }
$$

Para completar a analogia com o algoritmo de Euclides, e como conseqüência completar também a descrição de um algoritmo para verificar se uma equação linear arbitrária admite solução inteira, vamos estender o lema 3.1. Podemos observar que, se $\xi_{1}, \ldots, \xi_{m}$ satisfazem (3.2), então satisfazem

$$
\alpha_{1} \xi_{1}+\left(\alpha_{2}+\mu_{2} \alpha_{1}-\mu_{2} \alpha_{1}\right) \xi_{2}+\cdots+\left(\alpha_{m}+\mu_{m} \alpha_{1}-\mu_{m} \alpha_{1}\right) \xi_{m}=\beta
$$

para quaisquer $\mu_{2}, \ldots, \mu_{m}$. Por outro lado, essa igualdade pode ser reescrita da seguinte maneira:

$$
\alpha_{1}\left(\xi_{1}-\mu_{2} \xi_{2}-\cdots-\mu_{m} \xi_{m}\right)+\left(\alpha_{2}+\mu_{2} \alpha_{1}\right) \xi_{2}+\cdots+\left(\alpha_{m}+\mu_{m} \alpha_{1}\right) \xi_{m}=\beta .
$$

Se supormos que $\mu_{2}, \ldots, \mu_{m}$ são inteiros, temos que $\xi_{1}-\mu_{2} \xi_{2}-\cdots-\mu_{m} \xi_{m}$ também é inteiro. Logo, se a equação linear (3.2) admite solução inteira, então o mesmo pode ser dito a respeito da equação

$$
\alpha_{1} \xi_{1}+\left(\alpha_{2}+\mu_{2} \alpha_{1}\right) \xi_{2}+\cdots+\left(\alpha_{m}+\mu_{m} \alpha_{1}\right) \xi_{m}=\beta .
$$

A recíproca também é verdadeira: se $\xi_{1}, \ldots, \xi_{m}$ satisfazem a equação (3.5), podemos verificar que

$$
\alpha_{1}\left(\xi_{1}+\mu_{2} \xi_{2}+\cdots+\mu_{m} \xi_{m}\right)+\alpha_{2} \xi_{2}+\cdots+\alpha_{m} \xi_{m}=\beta
$$


Assim, podemos concluir que (3.2) admite solução inteira se e somente se (3.5) admite solução inteira. Ademais, se $\alpha_{1}, \ldots, \alpha_{m} \geq 0$ e $\alpha_{1}>0$, então $\alpha_{i}-\left\lfloor\alpha_{i} / \alpha_{1}\right\rfloor \alpha_{1} \geq 0$ para todo $i$. Por fim, se $\alpha_{1}>0$ e $\alpha_{k}>0$, então $\alpha_{k}-\left\lfloor\alpha_{k} / \alpha_{1}\right\rfloor \alpha_{1}<\alpha_{k}$. Logo, para reduzir a equação original ao caso em que $\alpha_{k} \neq 0$ para um único $k$, basta executarmos uma versão generalizada do algoritmo de Euclides:

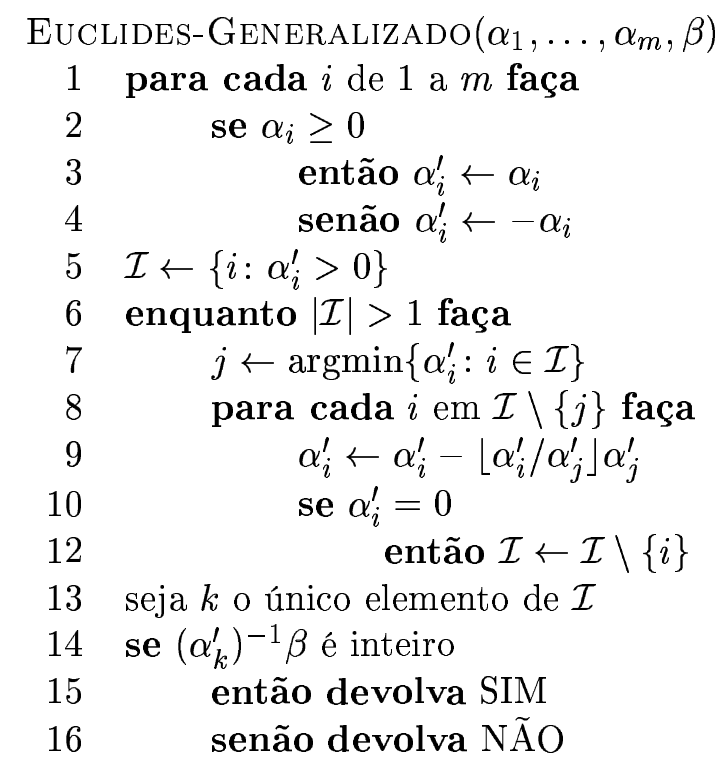

Como verificamos anteriormente, temos que, se $\alpha_{1}>0$ e $\alpha_{k}>0$, então $\alpha_{k}-\left\lfloor\alpha_{k} / \alpha_{1}\right\rfloor \alpha_{1}<\alpha_{k} / 2$. Logo, o número de iterações do algoritmo é limitado superiormente por $\log \left|\alpha_{1}\right|+\ldots+\log \left|\alpha_{m}\right|$. Temos um algoritmo polinomial para verificar se uma equação linear diofantina admite solução.

\subsection{Sistemas lineares diofantinos}

Vamos considerar uma generalização para equações lineares diofantinas. Seja $A \in \mathbb{Q}^{n \times m}$ uma matriz e seja $b \in \mathbb{Q}^{n}$ um vetor. Suponha que queremos obter um vetor $x$ tal que

$$
A x=b \quad \text { e } \quad x \in \mathbb{Z}^{m} .
$$

Uma restrição na forma acima é chamada de sistema de equações lineares diofantinas ou simplesmente sistema linear diofantino. Uma solução para (3.6) é uma solução inteira para

$$
A x=b .
$$

Considere o problema de verificar se um sistema linear admite solução inteira. Podemos então reformular esse problema da seguinte maneira: vamos denotar por $a_{i}$ a coluna $i$ de $A$. Nesse caso, temos que (3.7) admite solução inteira se e somente se existem inteiros $\xi_{1}, \ldots, \xi_{m}$ tais que

$$
a_{1} \xi_{1}+\cdots+a_{m} \xi_{m}=b
$$

Temos então uma proximidade de formulação entre equações lineares diofantinas e sistemas lineares diofantinos, o que torna natural a idéia de estendermos os argumentos vistos anteriormente para verificar se uma equação linear diofantina admite solução. 
Primeiramente, vamos aplicar a idéia de desconsiderar o caso em que o sistema não admite nenhuma solução. Vamos relembrar o algoritmo de eliminação gaussiana: através dele, podemos verificar se o sistema $A x=b$ admite solução e, em caso positivo, obter uma matriz $Q$ e um sistema

$$
\left[\begin{array}{ll}
D E & E
\end{array} x^{\prime}=c \text {, onde } D\right. \text { é uma matriz diagonal não-singular, }
$$

tais que $x^{\prime}:=Q x$ é uma solução de (3.8) se $x$ é uma solução de (3.7) e $x:=Q^{-1} x^{\prime}$ é uma solução de (3.7) se $x^{\prime}$ é uma solução de (3.8). Ademais, temos garantia de que as matrizes $Q$ e $Q^{-1}$ são inteiras, o que implica que $A x=b$ admite solução inteira se e somente se $[D E] x^{\prime}=c$ admite solução inteira. Alternativamente, essa propriedade pode ser vista como conseqüência do lema a seguir:

Lema 3.4. Seja $A$ uma matriz, seja $b$ um vetor e seja $Q$ uma matriz não-singular. Se $Q$ e $Q^{-1}$ são matrizes inteiras, então o sistema $A x=b$ admite solução inteira se e somente se o sistema $A Q x^{\prime}=b$ admite solução inteira.

Demonstração. Se $x$ satisfaz $A x=b$, então $x^{\prime}:=Q^{-1} x$ satisfaz $A Q x^{\prime}=b$. Se $x^{\prime}$ satisfaz $A Q x^{\prime}=b$, então $x:=Q x^{\prime}$ satisfaz $A x=b$.

A partir desse lema podemos observar que, se executarmos sobre a matriz $A$ uma operação que equivale a multiplicar essa matriz por alguma matriz não-singular $Q$ tal que $Q$ e $Q^{-1}$ são matrizes inteiras, obtemos um sistema equivalente em relação à existência de solução. Sabemos também que uma operação elementar sobre colunas corresponde precisamente a esse tipo de operação se for

(i) permutação de colunas;

(ii) multiplicação de uma coluna por -1 ;

(iii) soma do múltiplo inteiro de uma coluna a outra.

Vamos chamar esses três tipos de operações elementares de operações diofantinas sobre colunas.

Cabe observar que, para o caso particular em que o sistema contém apenas uma equação linear, as operações (ii) e (iii) correspondem precisamente às operações feitas sobre $\alpha_{1}, \ldots, \alpha_{m}$ ao longo do algoritmo EuClides-Generalizado. São, portanto, extensões naturais dessas operações.

Vamos então descrever a versão para sistemas do caso em que, para uma única equação linear, $\alpha_{k} \neq 0$ para um único $k$. Sob o ponto de vista de matrizes, podemos definir a seguinte generalização dessa condição: existe uma única submatriz $G$ de $A$ tal que $G$ é não-singular. Mais especificamente,

$$
A=\left[\begin{array}{ll}
G & 0
\end{array}\right] \text {, onde } G \text { é uma matriz não-singular. }
$$

Nesse caso temos que, se $G^{-1} b$ é um vetor inteiro, uma solução inteira para $A x=b$ é dada por

$$
x:=\left(\begin{array}{c}
G^{-1} b \\
0
\end{array}\right)
$$

Por outro lado, se $G^{-1} b$ não é um vetor inteiro, então existe uma linha de $G^{-1}$ que, multiplicada por $b$, não resulta em um inteiro. Vamos denotar essa linha por $y$. Sabemos que $y A$ é inteiro, pois

$$
G^{-1} A=G^{-1}\left[\begin{array}{ll}
G & 0
\end{array}\right]=\left[\begin{array}{ll}
I & 0
\end{array}\right] .
$$

Logo, podemos concluir que não existe solução inteira para $A x=b$ devido ao resultado abaixo: 
Lema 3.5. Seja $A$ uma matriz e seja $b$ um vetor. Se existe um vetor y tal que yA é inteiro e yb não é inteiro, então não existe um vetor inteiro $x$ tal que $A x=b$.

Demonstração. Suponha que tal $x$ existe. Então $y b=y(A x)=(y A) x$ é inteiro, uma contradição.

Podemos então deduzir a partir desses dois casos uma extensão natural da equivalência (3.4): existe solução inteira se e somente se $G^{-1} b$ é inteiro.

Para podermos descrever um algoritmo completo, resta então obter um procedimento para reduzir uma matriz a esse caso simples. Tal procedimento, no entanto, não precisa considerar a matriz de entrada como sendo totalmente arbitrária: podemos verificar que uma matriz na forma (3.8) tem posto linha completo. Ademais, sabemos que $x$ satisfaz $A x=b$ se e somente se

$$
\delta A x=\delta b
$$

para todo $\delta$. Em particular, podemos supor que $\delta A$ é uma matriz inteira. Vamos então supor sem perda de generalidade que a matriz inicial é inteira. Além disso, podemos supor que a matriz tem posto linha completo devido à eliminação gaussiana utilizada como pré-processamento.

A forma (3.9) pode ser considerada como uma "versão transposta" da matriz que a eliminação gaussiana visa obter. Vamos, portanto, utilizar a mesma estratégia da primeira fase da eliminação: triangularizar a matriz através de operações elementares. Nesse caso específico, operações diofantinas sobre colunas. Uma matriz inteira de posto linha completo está na forma $G_{k}$ se puder ser escrita como

$$
\left[\begin{array}{cc}
G & 0 \\
V & W
\end{array}\right] \text {, onde } G \text { é uma matriz triangular inferior não-singular inteira de ordem } k \text {. }
$$

Podemos observar que, se $A$ é uma matriz em $\mathbb{Q}^{n \times m}$ na forma $G_{k}$, então $k=n$ implica que $A$ está na forma (3.9). Ademais, como não impomos nenhuma restrição sobre a matriz $W$ além da integralidade, temos que qualquer matriz inteira com posto linha completo está na forma $G_{0}$. Logo, para termos o algoritmo desejado basta descrever um procedimento que, dada uma matriz inteira $A$ em $\mathbb{R}^{n \times m}$ na forma $G_{k}$ tal que $k<n$, a transforma em uma matriz na forma $G_{k+1}$.

Como vimos anteriormente, devemos utilizar operações diofantinas sobre colunas no desejado procedimento. Cabe observar que qualquer operação elementar sobre as $m-k$ últimas colunas de $A$ não tem nenhum efeito sobre as $k$ primeiras linhas da matriz. Cabe também observar que a primeira linha de $W$ contém pelo menos um elemento não-nulo, pois caso contrário as linhas da matriz

$$
\left[\begin{array}{cc}
G & 0 \\
v & w
\end{array}\right], \text { onde }\left\{\begin{array}{l}
v \text { é a primeira linha de } V \\
w \text { é a primeira linha de } W
\end{array}\right.
$$

formariam um conjunto linearmente dependente, uma contradição com o posto linha completo da matriz. Por fim, cabe observar que operações elementares sobre colunas preservam o posto linha completo de uma matriz, visto que equivalem à multiplicação por matriz não-singular.

Assim, é suficiente descrever um procedimento que, dada uma matriz inteira $W$ cuja primeira linha contém pelo menos um elemento não-nulo, transforma essa matriz, utilizando apenas operações diofantinas sobre colunas, em uma matriz inteira na forma

$$
\left[\begin{array}{cc}
\omega & 0 \\
w^{\prime} & W^{\prime}
\end{array}\right] \text {, onde } \omega \text { é não-nulo. }
$$


Como estamos impondo restrições sobre a primeira linha da matriz, um procedimento que se encaixa nesse propósito é precisamente uma versão estendida do algoritmo de Euclides: basta aplicar as operações diofantinas sobre colunas que, quando restritas à primeira linha, correspondem às operações que o algoritmo EuCLIDES-GENERALIZADo faria. E para que o elemento não-nulo esteja na primeira coluna no final do procedimento, basta uma simples permutação.

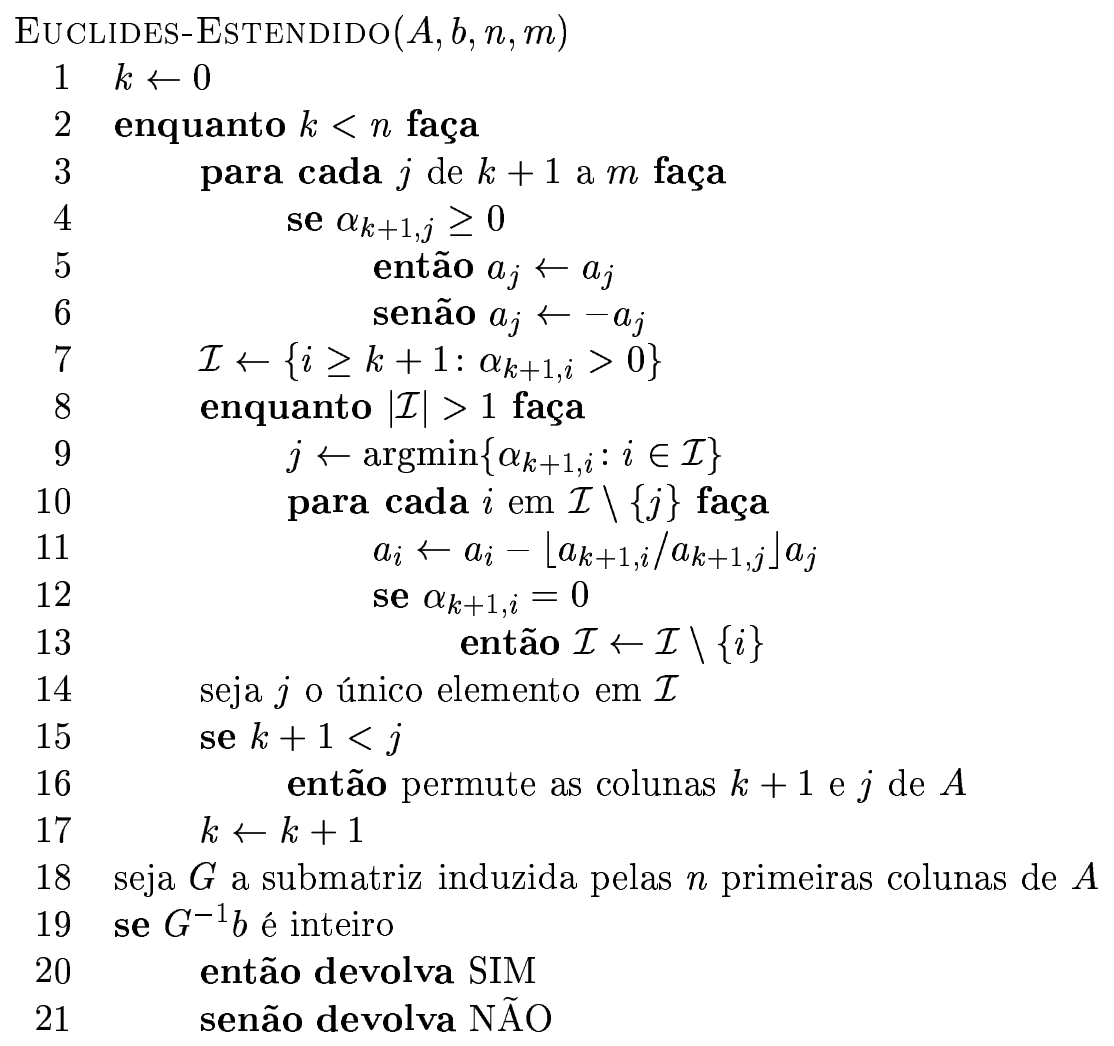

Temos então um algoritmo para verificar se um dado sistema linear admite solução inteira.

\subsection{Forma normal de Hermite}

Utilizando o argumento de que, se $\alpha_{1}>0$ e $\alpha_{k}>0$, então $\alpha_{k}-\left\lfloor\alpha_{k} / \alpha_{1}\right\rfloor \alpha_{1}<\alpha_{k} / 2$, podemos verificar que o número de operações diofantinas que são executadas ao longo de uma iteração do algoritmo EuCLIDES-ESTENDIDO é limitado superiormente por $\log \left(\alpha_{k+1, k+1}\right)+\cdots+\log \left(\alpha_{k+1, n}\right)$. Entretanto, esse é um limitante local, que à primeira vista depende não do tamanho da matriz inicial, mas do tamanho da matriz no início dessa iteração específica.

Para que tenhamos um algoritmo polinomial, precisamos garantir que o tamanho de todas as matrizes obtidas ao longo do algoritmo é limitado polinomialmente pelo tamanho da matriz inicial. Um método simples para se atingir esse objetivo é reduzir explicitamente o tamanho dos elementos durante as iterações, através de operações específicas para esse propósito.

Sejam $\alpha_{1}$ e $\alpha_{2}$ inteiros tais que $\alpha_{2}>0$. Aplicar a operação de reduzir $\alpha_{1}$ módulo $\alpha_{2}$ significa substituir $\alpha_{1}$ por $\alpha_{1} \bmod \alpha_{2}$. Em termos algorítmicos, significa executar a seguinte operação:

- se $\alpha_{1} \geq 0$, subtraia $\left\lfloor\alpha_{1} / \alpha_{2}\right\rfloor \alpha_{2}$ de $\alpha_{1}$; 
- se $\alpha_{1}<0$, some $\left\lceil\alpha_{1} / \alpha_{2}\right\rceil \alpha_{2}$ a $\alpha_{1}$.

Para saber como podemos utilizar esse conceito para reduzir o tamanho das matrizes ao longo do algoritmo, vamos primeiramente considerar o caso particular no qual existe um inteiro $\mu$ tal que todas as colunas da matriz $\mu I$ pertencem a $A$. Nesse caso específico, um elemento de $A$ pode ser reduzido módulo $\mu$ através de uma operação diofantina sobre as colunas de $A$. De fato, sendo $e_{i}$ o vetor canônico relativo a $i$, uma redução de $\alpha_{i, j}$ módulo $\mu$ pode ser obtida através da operação

- se $\alpha_{i, j} \geq 0$, subtraia $\left\lfloor\alpha_{i, j} / \mu\right\rfloor \mu e_{i}$ de $a_{j}$;

- se $\alpha_{i, j}<0$, some $\left\lceil\alpha_{i, j} / \mu\right\rceil \mu e_{i}$ a $a_{j}$

se supormos que $\mu e_{i}$, a coluna $i$ de $\mu I$, pertence a $A$. Logo, sob a hipótese de que dentre as colunas de $A$ estão todas as colunas de $\mu I$, podemos modificar o algoritmo EuCLIDES-ESTEndido de forma a limitar polinomialmente o tamanho dos elementos da matriz pelo tamanho de $\mu$. Em particular, se o tamanho de $\mu$ é polinomialmente limitado pelo tamanho de $A$, essa modificação é suficiente para obtermos um algoritmo polinomial que decide se um sistema diofantino tem solução.

A hipótese de que $A$ contém as colunas de $\mu I$, no entanto, é muito forte. Vamos então considerar a possibilidade de adicionar essas colunas a $A$ sem influenciar a existência de solução. Seja $a$ um vetor tal que $A z=a$ para algum $z$ inteiro. Se para um dado $b$ temos que $A x=b$ para $x$ inteiro, então $[A a] x^{\prime}=b$ admite solução inteira, mais especificamente $x^{\prime}:=(x 0)$. Por outro lado, se $[A a] x^{\prime}=b$ para um dado $x^{\prime}$ inteiro, onde $x^{\prime}=(x \xi)$, então $A x+\xi A z=b$, ou seja, $x+\xi z$ é uma solução inteira para $A x=b$. Podemos concluir então que, para o problema de decisão, a adição de $a$ ao conjunto de colunas de $A$ não tem nenhum efeito.

Portanto, é interessante obter um inteiro $\mu$ de tamanho polinomialmente limitado pelo tamanho de $A$ tal que, para toda coluna $\mu e_{i}$ de $\mu I$, existe solução inteira para $A x=\mu e_{i}$. O lema a seguir mostra que um dos inteiros que satisfazem tais condições é determinante de uma submatriz de $A$.

Lema 3.6. Seja A uma matriz inteira com posto linha completo, seja n o número de linhas dessa matriz e seja $\mu$ o determinante de uma submatriz não-singular de ordem $n$ de $A$. Para todo vetor canônico $e_{i}$, existe solução inteira para o sistema $A x=\mu e_{i}$.

Demonstração. Seja $G$ uma submatriz não-singular de ordem $n \operatorname{de} A$ e seja $\mu$ o determinante de $G$. Sabemos por Laplace que todos os elementos de $G^{-1}$ são o resultado da divisão de um inteiro por $\operatorname{det}(G)$. Logo, podemos concluir que a matriz $\mu G^{-1}$ é inteira. Portanto, $G \mu G^{-1}=\mu G G^{-1}=\mu I$, o que implica que, para cada $i$, o vetor $\mu e_{i}$ é dado pela multiplicação de $G$ pela coluna $i$ de $\mu G^{-1}$.

Como estamos supondo que $A$ tem posto linha completo, submatrizes não-singulares cuja ordem é o número de linhas de $A$ existem e o determinante $\mu$ de alguma delas pode ser obtido em tempo polinomial se utilizarmos eliminação gaussiana. Esse passo completa o seguinte algoritmo polinomial:

(i) calcule com eliminação gaussiana o determinante $\mu$ de uma submatriz de ordem $n$ qualquer da matriz $A$, onde $n$ é o número de linhas de $A$;

(ii) aplique o algoritmo EuCLIDES-Estendido sobre a matriz $[A \mu I]$;

(iii) após cada iteração $k$ do algoritmo EuCLIDES-Estendido, utilize as $n-k$ últimas colunas da matriz obtida para reduzir módulo $\mu$ todos os elementos das $n-k$ últimas linhas dessa matriz. 
Antes de apresentar essa versão modificada do EuCLIDES-EsTEnDIDO em pseudo-código, vamos adicionar a ela um refinamento extra muito interessante. Sejam $[G 0]$ e $\left[G^{\prime} 0\right]$ as matrizes obtidas quando esse algoritmo é aplicado sobre $A$ e $[A \mu I]$, respectivamente. Sabemos que $G$ e $G^{\prime}$ são ambas inteiras, triangulares inferiores e não-singulares. No entanto, o algoritmo EuCLIDES-ESTENDIDO não garante que $G$ e $G^{\prime}$ são iguais. Se essa garantia existisse, poderíamos concluir que a substituição da matriz $A$ pela matriz $[A \mu I]$ não tem nenhum efeito sobre a matriz obtida ao final do algoritmo, salvo a presença de algumas colunas nulas a mais.

Surpreendentemente, essa igualdade é garantida se exigirmos apenas mais uma propriedade sobre as matrizes $G$ e $G^{\prime}$. Vamos denotar por $\gamma_{i, j}$ o elemento na linha $i$ e coluna $j$ de uma matriz $H$. Dizemos que uma matriz está na forma normal de Hermite se ela possui a seguinte estrutura:

$$
\left[\begin{array}{ll}
H & 0
\end{array}\right] \text {, onde } H \text { é uma matriz }\left\{\begin{array}{l}
\text { inteira e não-negativa; } \\
\text { triangular inferior não-singular; } \\
\text { tal que, para quaisquer } i \neq j, \text { vale } \gamma_{i, i}>\gamma_{i, j}
\end{array}\right.
$$

Mais informalmente, um elemento na diagonal de $H$ é o maior elemento da linha à qual pertence. $\mathrm{O}$ teorema a seguir relaciona matrizes na forma normal de Hermite à igualdade que mencionamos:

Teorema 3.7. Sejam $\left[\begin{array}{ll}H & 0\end{array}\right]$ e $\left[\begin{array}{ll}H^{\prime} & 0\end{array}\right]$ duas matrizes na forma normal de Hermite. As matrizes $H$ e $H^{\prime}$ são iguais se e somente se, para todo vetor $b$, o sistema $[H \quad 0] x=b$ admite solução inteira se $e$ somente se o sistema $\left[H^{\prime} 0\right] x=b$ admite solução inteira.

Demonstração. Como a recíproca é trivial, vamos apenas demonstrar que $H=H^{\prime}$ se, para todo vetor $b$, o sistema $\left[\begin{array}{ll}H & 0\end{array}\right] x=b$ admite solução inteira se e somente se o sistema $\left[H^{\prime} 0\right] x=b$ admite solução inteira. Suponha por contradição que essa hipótese vale mas $H \neq H^{\prime}$. Em particular, vamos considerar $i$ e $j$ tais que $\gamma_{i, j} \neq \gamma_{i, j}^{\prime}$ e $i$ é mínimo. Como $H$ é triangular inferior, temos $i \geq j$.

Vamos denotar por $h_{j}$ e $h_{j}^{\prime}$ as colunas $j$ de $H$ e $H^{\prime}$, respectivamente. Sabemos que existe solução inteira para $\left[\begin{array}{ll}H & 0\end{array}\right] x=h_{j}$ e $\left[\begin{array}{ll}H^{\prime} & 0\end{array}\right] x=h_{j}^{\prime}$. Logo, existe $x$ inteiro tal que $H x=h_{j}-h_{j}^{\prime}$. Denotando por $\xi_{i}$ o elemento $i$ de $x$, seja $k$ um índice tal que $\xi_{k} \neq 0$ e $k$ é o menor possível. A existência de $k$ é garantida pela hipótese $\gamma_{i, j} \neq \gamma_{i, j}^{\prime}$, pois ela implica que $h_{j}-h_{j}^{\prime} \neq 0$. Como $H$ é uma matriz triangular inferior não-singular, a minimalidade do índice $k$ garante que $k=i$. A forma da matriz $H$ e a minimalidade de $k$ também garantem que $\left|\gamma_{k, j}-\gamma_{k, j}^{\prime}\right|=\left|\xi_{k} \gamma_{k, k}\right|$. Como $k=i$, segue que $\left|\gamma_{i, j}-\gamma_{i, j}^{\prime}\right|=\left|\xi_{i} \gamma_{i, i}\right|$. Podemos concluir que $\left|\gamma_{i, j}-\gamma_{i, j}^{\prime}\right| \geq \gamma_{i, i}$, pois $\xi_{i}$ é inteiro.

Como $H$ e $H^{\prime}$ são matrizes na forma normal de Hermite, temos que $\gamma_{i, j}, \gamma_{i, j}^{\prime} \geq 0$. Supondo sem perda de generalidade que $\gamma_{i, j} \geq \gamma_{i, j}^{\prime}$, podemos verificar que $\left|\gamma_{i, j}-\gamma_{i, j}^{\prime}\right|=\gamma_{i, j}-\gamma_{i, j}^{\prime} \leq \gamma_{i, j}$. Ademais, a forma normal de Hermite também implica que $\gamma_{i, j}<\gamma_{i, i}$ se $i>j$. Portanto, temos $i \leq j$, pois caso contrário iríamos concluir que $\left|\gamma_{i, j}-\gamma_{i, j}^{\prime}\right|<\gamma_{i, i}$, uma contradição.

Podemos então concluir que $i=j$, o que implica $\left|\gamma_{i, i}-\gamma_{i, i}^{\prime}\right| \geq \gamma_{i, i}$. Como $H$ está na forma normal de Hermite, temos $\gamma_{i, i}, \gamma_{i, i}^{\prime}>0$. Portanto, como por hipótese sabemos que $\gamma_{i, i} \geq \gamma_{i, i}^{\prime}$, concluímos $\gamma_{i, i}-\gamma_{i, i}^{\prime}<\gamma_{i, i}$. Temos novamente uma contradição e podemos concluir que $H$ e $H^{\prime}$ são iguais.

O pseudo-código a seguir é uma versão do algoritmo EuClidEs-EsTEnDIDO que considera as modificações mencionadas anteriormente e também transforma a matriz dada na entrada em uma matriz na forma normal de Hermite, ao invés de simplesmente transformá-la em uma matriz inteira, triangular inferior e não-singular. Supõe-se que o inteiro $\mu$ já foi obtido em um pré-processamento. 


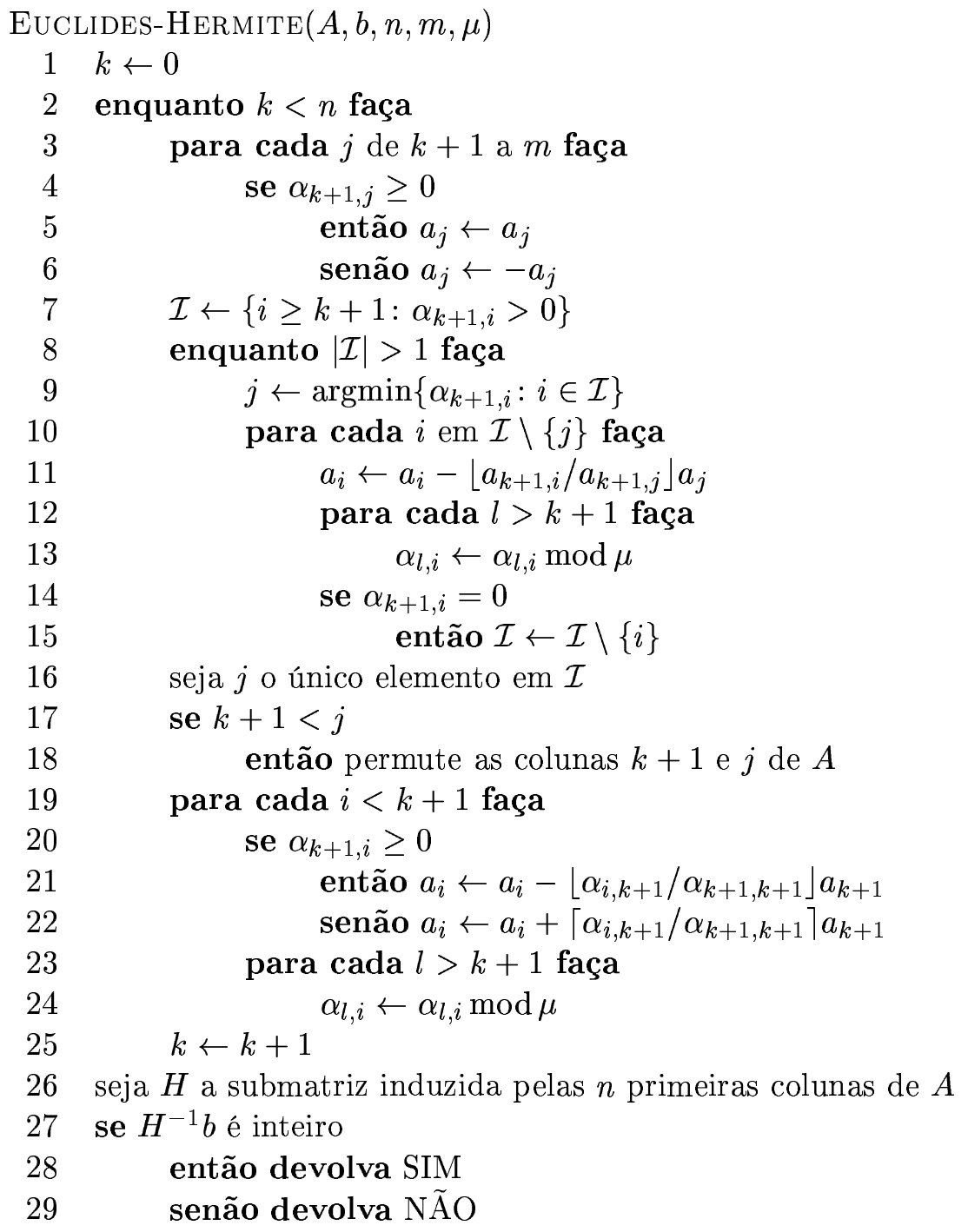

Temos então um algoritmo polinomial para transformar uma matriz com posto linha completo em uma matriz na forma normal de Hermite através de operações diofantinas sobre colunas. Logo:

Corolário 3.8. Seja A uma matriz com posto linha completo. Existe uma única matriz na forma normal de Hermite $\left[\begin{array}{ll}H & 0\end{array}\right]$ tal que, para todo b, o sistema $A x=b$ admite solução inteira se e somente se $\left[\begin{array}{ll}H & 0\end{array}\right] x=b$ admite solução inteira. Ademais, essa matriz pode ser obtida em tempo polinomial.

Demonstração. Segue do teorema 3.7 e da correção do algoritmo EuClides-HeRmite.

Podemos nos referir à matriz do corolário como a forma normal de Hermite da dada matriz $A$.

\subsection{Matrizes unimodulares}

Comparando o algoritmo EuCLIDES-HeRmite com a eliminação gaussiana, podemos observar que no primeiro houve uma omissão: não está incluído no algoritmo o cálculo da matriz representando as operações diofantinas sobre colunas, que nesta seção chamaremos de $U$. À primeira vista, essa 
parece ser uma omissão grave, visto o papel fundamental que as matrizes de operações elementares têm na eliminação gaussiana. O papel da matriz $U$ é igualmente importante para sistemas lineares diofantinos. A diferença nesse caso é que ela pode ser calculada a posteriori.

Consideremos primeiro o caso particular no qual a matriz $A$ é não-singular. Nesse caso, a forma normal de Hermite dessa matriz é dada por uma matriz também não-singular $H$. Como estamos supondo que $A$ é inversível, temos que $U:=A^{-1} H$ é a única matriz tal que $A U=H$.

Vamos então tentar generalizar essa construção para o caso em que $A$ não é necessariamente não-singular. Como estamos supondo que $A$ ao menos tem posto linha completo, sabemos que seu número de linhas é menor que seu número de colunas. Ademais, sabemos que a dimensão de $U$ é dada pelo número de colunas de $A$. Logo, podemos considerar a seguinte idéia: adicionar novas linhas à matriz $A$ com o objetivo de obter uma matriz não-singular.

Considere a transposta $A^{\prime}$ de $A$. Como $A$ tem posto linha completo, $A^{\prime}$ tem posto coluna completo. Sabemos pelo teorema 1.13 que, após aplicar a eliminação gaussiana sobre $A^{\prime}$, obtemos uma matriz $P$ e um inteiro $r$ tal que as $r$ primeiras linhas de $P A^{\prime}$ formam um subconjunto linearmente independente maximal das linhas de $A^{\prime}$. Como $A^{\prime}$, tem posto coluna completo, concluímos que

$$
P A^{\prime}=\left[\begin{array}{c}
A_{1} \\
A_{2}
\end{array}\right] \text {, onde } A_{1} \text { é uma matriz não-singular. }
$$

Sejam $P^{\prime}, A_{1}^{\prime}, A_{2}^{\prime}$ as transpostas de $P, A_{1}^{\prime}, A_{2}^{\prime}$ respectivamente. Como $A_{1}$ é matriz não-singular,

$$
\left[\begin{array}{cc}
A_{1}^{\prime} & A_{2}^{\prime} \\
0 & I
\end{array}\right]
$$

também é não-singular e a forma normal de Hermite dessa matriz maior possui a seguinte forma:

$\left[\begin{array}{cc}H & 0 \\ H^{\prime} & H^{\prime \prime}\end{array}\right]$, onde $\left[\begin{array}{ll}H & 0\end{array}\right]$ está na forma normal de Hermite e as ordens de $H$ e $A_{1}^{\prime}$ são iguais.

Podemos então observar que a matriz [ $\left.\begin{array}{ll}H & 0\end{array}\right]$ pode ser obtida a partir de $A$ da seguinte maneira:

$$
\begin{aligned}
A P^{\prime}\left[\begin{array}{cc}
A_{1}^{\prime} & A_{2}^{\prime} \\
0 & I
\end{array}\right]^{-1}\left[\begin{array}{cc}
H & 0 \\
H^{\prime} & H^{\prime \prime}
\end{array}\right] & =\left[\begin{array}{ll}
A_{1} & A_{2}
\end{array}\right]\left[\begin{array}{cc}
A_{1}^{\prime} & A_{2}^{\prime} \\
0 & I
\end{array}\right]^{-1}\left[\begin{array}{cc}
H & 0 \\
H^{\prime} & H^{\prime \prime}
\end{array}\right] \\
& =\left[\begin{array}{ll}
I & 0
\end{array}\right]\left[\begin{array}{cc}
H & 0 \\
H^{\prime} & H^{\prime \prime}
\end{array}\right] \\
& =\left[\begin{array}{ll}
H & 0
\end{array}\right] .
\end{aligned}
$$

Logo, uma matriz $U$ tal que $A U=\left[\begin{array}{ll}H & 0\end{array}\right]$ e $\left[\begin{array}{ll}H & 0\end{array}\right]$ está na forma normal de Hermite é dada por

$$
U:=P^{\prime}\left[\begin{array}{cc}
A_{1}^{\prime} & A_{2}^{\prime} \\
0 & I
\end{array}\right]^{-1}\left[\begin{array}{cc}
H & 0 \\
H^{\prime} & H^{\prime \prime}
\end{array}\right] .
$$

Podemos então concluir que o algoritmo Euclides-Hermite é suficiente para obter tanto a forma normal de Hermite de uma matriz como a matriz de transformação, bastando aplicá-lo sobre uma versão modificada da matriz original que pode ser obtida com a eliminação gaussiana. Portanto:

Teorema 3.9. Existe um algoritmo polinomial que, dada uma matriz $A$ e um vetor $b$, verifica se $A x=b$ admite solução inteira, devolve uma solução em caso positivo e devolve um vetor y tal que yA é inteiro e yb não é inteiro em caso negativo. 
Demonstração. Através da eliminação gaussiana e do algoritmo EuClidEs-HERMiTe, sabemos que todas as matrizes mencionadas podem ser calculadas. Se $H^{-1} b$ é um vetor inteiro, podemos utilizá-lo junto com a matriz $U$ para construir uma solução inteira de $A x=b$. Caso contrário, existe uma linha de $H^{-1}$ a partir da qual podemos construir um vetor $y$ que é o certificado de inexistência.

Como vimos anteriormente, a propriedade que nos permite aplicar operações diofantinas sobre $A$ é o fato de que cada uma de tais operações equivale à multiplicação por uma matriz $Q$ tal que $Q$ e $Q^{-1}$ são matrizes inteiras. Em particular, a mesma propriedade vale para a matriz $U$.

$\mathrm{Na}$ verdade, essa integralidade é conseqüência de uma propriedade mais forte. Sabemos que a matriz $Q$ que representa uma operação diofantina satisfaz $\operatorname{det}(Q)=1$ ou $\operatorname{det}(Q)=-1$. De maneira mais sucinta, $|\operatorname{det}(Q)|=1$. Podemos então observar que, se $A$ é uma matriz não-singular e $H$ é sua forma normal de Hermite, então $|\operatorname{det}(A)|=|\operatorname{det}(A U)|=|\operatorname{det}(H)|$. Logo, se $U=A^{-1} H$, então temos que, para a matriz $U$ que satisfaz $A U=H$, vale que $|\operatorname{det}(U)|=1$.

Dizemos que uma matriz $U$ é unimodular se $U$ é uma matriz inteira não-singular que satisfaz a propriedade $|\operatorname{det}(U)|=1$. O resultado a seguir mostra o papel da unimodularidade na inversa.

Lema 3.10. Se U é uma matriz unimodular, então $U^{-1}$ é uma matriz unimodular.

Demonstração. Sabemos que todo elemento de $U^{-1}$ é o quociente de um subdeterminante de $U$ por $\operatorname{det}(U)$. Sendo $U$ inteira, todo subdeterminante de $U$ é inteiro e $|\operatorname{det}(U)|=1$, estamos feitos.

Pela definição de unimodularidade, podemos verificar que o lema acima implica na propriedade que buscávamos: se $U$ é unimodular, então $U$ e $U^{-1}$ são matrizes inteiras.

A importância da unimodularidade, porém, não se restringe a essa propriedade. Considere a matriz identidade, o exemplo mais simples de matriz unimodular. Por definição, uma matriz identidade está na forma normal de Hermite. Uma observação interessante é que, além disso, ela é a única matriz unimodular na forma normal de Hermite.

Lema 3.11. Se $U$ é unimodular e $U$ está na formal normal de Hermite, então $U=I$.

Demonstração. Se $U$ está na formal de Hermite, então é uma matriz triangular inferior, o que implica que $\operatorname{det}(U)$ é o resultado da multiplicação dos elementos de sua diagonal. Sabemos ainda que esses elementos são inteiros não-negativos. Logo, se $U$ é unimodular, todos os elementos de sua diagonal são iguais a 1. Ademais, todos os elementos abaixo da diagonal são inteiros não-negativos menores do que 1 , ou seja são iguais a zero. Podemos então concluir que $U=I$.

Como verificamos anteriormente, o valor absoluto do determinante de uma matriz é igual ao valor absoluto do determinante de sua forma normal de Hermite. Logo,

Lema 3.12. U é unimodular se e somente se sua forma normal de Hermite é a identidade.

Demonstração. A forma normal de Hermite $H$ de uma matriz $U$ é unimodular se e somente se $U$ é unimodular. Mas a única matriz unimodular na forma normal de Hermite é a identidade.

Se $I$ é a forma normal de Hermite de uma matriz $A$, sabemos que, para todo vetor $b$, o sistema dado por $A x=b$ admite solução inteira se e somente se $I x=b$ admite solução inteira. Logo,

Corolário 3.13. Uma matriz $A$ é unimodular se e somente se, para todo b inteiro, existe solução inteira para o sistema $A x=b$. 
Demonstração. A é unimodular se e somente se sua forma normal de Hermite é $I$. Por outro lado, se $b$ é inteiro, então $I x=b$ admite solução inteira, o próprio $b$.

Podemos resumir todas as propriedades da unimodularidade em uma simples equivalência:

Teorema 3.14. As seguintes afirmações são equivalentes:

(i) U é uma matriz unimodular;

(ii) $U^{-1}$ é uma matriz unimodular;

(iii) a forma normal de Hermite de U é a identidade;

(iv) para todo b inteiro, o sistema $U x=b$ admite solução inteira.

\subsection{Reticulados e bases}

Sejam $a_{1}, \ldots, a_{m}$ vetores. O reticulado gerado por tais vetores é o conjunto dado por

$$
\operatorname{ret}\left(a_{1}, \ldots, a_{m}\right):=\left\{a: a=\lambda_{1} a_{1}+\cdots+\lambda_{m} a_{m}, \text { onde } \lambda_{1}, \ldots, \lambda_{m} \in \mathbb{Z}\right\} .
$$

Mais informalmente, é o conjunto de todos os vetores gerados por combinações inteiras de $a_{1}, \ldots, a_{m}$.

Seja $A$ uma matriz tal que $a_{i}$ é a coluna $i$ de $A$. Nesse caso, podemos verificar que um dado vetor $b$ está em $\operatorname{ret}\left(a_{1}, \ldots, a_{m}\right)$ se e somente se o sistema $A x=b$ admite solução inteira. Em caso positivo, os elementos de uma solução $x$ são os coeficientes da combinação inteira correspondente.

Teorema 3.15. Sejam $a_{1}, \ldots, a_{m}, b$ vetores. $O$ vetor $b$ pertence ao reticulado de $a_{1}, \ldots, a_{m}$ se $e$ somente se não existe um vetor y tal que yb não é inteiro e ya $a_{i}$ é inteiro para todo $i$.

Como conseqüência do algoritmo de Euclides, existe um algoritmo polinomial correspondente.

Teorema 3.16. Existe um algoritmo polinomial que, dados vetores $a_{1}, \ldots, a_{m}, b$, verifica se o vetor $b$ pertence ao reticulado de $a_{1}, \ldots, a_{m}$, devolve uma combinação linear inteira correspondente em caso positivo e devolve um vetor y tal que yb não é inteiro e ya $a_{i}$ é inteiro para todo i em caso negativo.

Geometricamente, podemos visualizar um reticulado como sendo uma "grade", caracterizada por conjuntos de hiperplanos paralelos. Se um dado vetor não pertence a algum desses conjuntos, então ele não pertence ao reticulado. A figura 3.1 mostra um exemplo dessa interpretação.

De maneira análoga ao que foi definido para subespaços, dizemos que a base de um reticulado é um conjunto linearmente independente que o gera. Através do algoritmo estendido de Euclides, podemos mostrar que todo reticulado admite uma base que pode ser construída em tempo polinomial.

Teorema 3.17. Existe um algoritmo polinomial que, dado um conjunto de vetores, devolve uma base para o reticulado gerado por esse conjunto.

Demonstração. Sejam $a_{1}, \ldots, a_{m}$ vetores e seja $A$ a matriz cujas colunas são esses vetores. Após aplicarmos a eliminação gaussiana sobre $A$, obtemos matrizes $S, P, Q$ para as quais vale a igualdade

$$
S P A Q=\left[\begin{array}{cc}
D & E \\
0 & 0
\end{array}\right] \text {, onde } D \text { é uma matriz diagonal não-singular. }
$$




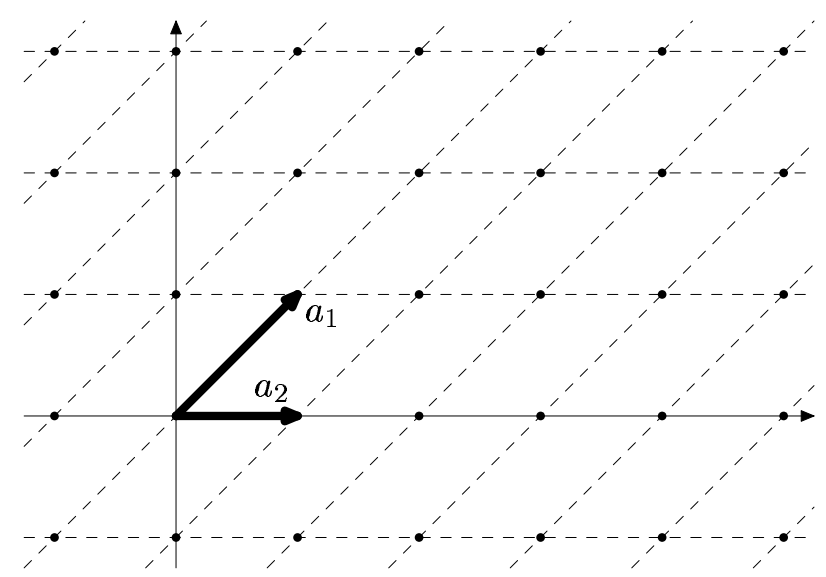

Figura 3.1: reticulado gerado pelos vetores $a_{1}, a_{2}$.

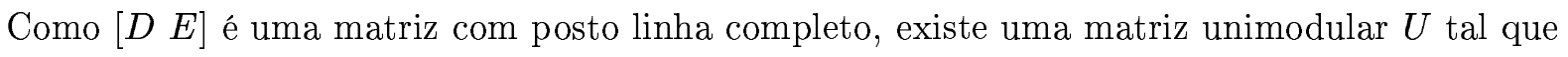

$$
S P A Q U=\left[\begin{array}{cc}
H & 0 \\
0 & 0
\end{array}\right] \text {, onde }\left[\begin{array}{ll}
H & 0
\end{array}\right] \text { é uma matriz na forma normal de Hermite. }
$$

Temos então que a matriz $A Q U=(S P)^{-1} S P A Q U$ está na forma $\left[A^{\prime} 0\right]$, onde $A^{\prime}$ é uma matriz cujo conjunto de colunas é linearmente independente. Ademais, como vimos que $Q U$ é unimodular, podemos verificar que $\left[A^{\prime} 0\right] x=b$ admite solução inteira se e somente se $A x=b$ admite solução inteira. Logo, o reticulado gerado pelas colunas de $A$ também é gerado pelas colunas de $A^{\prime}$.

Embora esse teorema prove que, dados vetores $a_{1}, \ldots, a_{m}$, podemos obter em tempo polinomial uma base de $\operatorname{ret}\left(a_{1}, \ldots, a_{m}\right)$, ele não é tão forte quanto o resultado que obtivemos para subespaços, visto que os vetores de tal base não necessariamente estão dentre os vetores originais $a_{1}, \ldots, a_{m}$. Um exemplo simples desse fato pode ser visto em dimensão 1: considere o reticulado gerado pelos inteiros 2 e 3 . Como $3-2=1$, tal reticulado é $\mathbb{Z}$. No entanto, nem 2 nem 3 podem gerar $\mathbb{Z}$.

A descrição dos algoritmos apresentados neste capítulo foi baseada no livro de SchriJVER [1986]. $\mathrm{O}$ autor desse livro cita muitas referências de diversos autores a respeito das origens de algoritmos polinomiais para sistemas lineares diofantinos. Em particular, menciona que a variante específica que descrevemos foi apresentada por Domich [1983] e Domich, Kannan E Trotter [1985]. 



\section{Capítulo 4}

\section{Cones inteiros e bases de Hilbert}

Neste capítulo, vamos apresentar o conceito de sistemas geradores de Hilbert e o conceito de bases de Hilbert, que buscam descrever a interseç̧ão dos "resultados não-negativos" e "resultados inteiros" que foram apresentados nos capítulos 2 e 3, respectivamente. Mostraremos que esses conjuntos especiais podem ser vistos como casos tratáveis de cones inteiros.

As definiçõ es que utilizaremos são diferentes da definição original de base de Hilbert na literatura. As duas últimas seções deste capítulo são dedicados à versão original e à relação entre as duas versões.

\subsection{Cones inteiros}

Vamos recapitular os três tipos de conjuntos gerados que vimos nos capítulos anteriores:

$$
\begin{aligned}
\operatorname{lin}\left(a_{1}, \ldots, a_{m}\right) & :=\left\{a: a=\lambda_{1} a_{1}+\cdots+\lambda_{m} a_{m}, \text { onde } \lambda_{1}, \ldots, \lambda_{m} \in \mathbb{R}\right\} . \\
\operatorname{cone}\left(a_{1}, \ldots, a_{m}\right) & :=\left\{a: a=\lambda_{1} a_{1}+\cdots+\lambda_{m} a_{m}, \text { onde } \lambda_{1}, \ldots, \lambda_{m} \in \mathbb{R}_{\geq}\right\} . \\
\operatorname{ret}\left(a_{1}, \ldots, a_{m}\right) & :=\left\{a: a=\lambda_{1} a_{1}+\cdots+\lambda_{m} a_{m}, \text { onde } \lambda_{1}, \ldots, \lambda_{m} \in \mathbb{Z}\right\} .
\end{aligned}
$$

Sobre esses três tipos de conjunto, apresentamos os seguintes teoremas de alternativa:

Teorema 1.11. Sejam $a_{1}, \ldots, a_{m}, b$ vetores. O vetor $b$ pertence ao fecho linear de $a_{1}, \ldots, a_{m}$ se $e$ somente se não existe um vetor y tal que $y b \neq 0$ e ya $a_{i}=0$ para todo $i$.

Teorema 2.22. Sejam $a_{1}, \ldots, a_{m}, b$ vetores. $O$ vetor $b$ pertence ao cone de $a_{1}, \ldots, a_{m}$ se e somente se não existe um vetor y tal que $y b<0$ e ya $a_{i} \geq 0$ para todo $i$.

Teorema 3.15. Sejam $a_{1}, \ldots, a_{m}, b$ vetores. O vetor $b$ pertence ao reticulado de $a_{1}, \ldots, a_{m}$ se $e$ somente se não existe um vetor y tal que yb não é inteiro e ya $a_{i}$ é inteiro para todo $i$.

Mais do que isso, os objetos acima podem ser todos obtidos de forma eficiente:

Teorema 1.12. Existe um algoritmo polinomial que, dados vetores $a_{1}, \ldots, a_{m}, b$, verifica se o vetor $b$ pertence ao fecho linear de $a_{1}, \ldots, a_{m}$, devolve a combinação linear correspondente em caso positivo e devolve um vetor y tal que $y b \neq 0$ e ya $a_{i}=0$ para todo $i$ em caso negativo.

Teorema 2.23. Existe um algoritmo polinomial que, dados vetores $a_{1}, \ldots, a_{m}, b$, verifica se o vetor $b$ pertence ao cone de $a_{1}, \ldots, a_{m}$, devolve a combinação linear não-negativa correspondente em caso positivo e devolve um vetor y tal que $y b<0$ e ya $a_{i} \geq 0$ para todo $i$ em caso negativo. 
Teorema 3.16. Existe um algoritmo polinomial que, dados vetores $a_{1}, \ldots, a_{m}, b$, verifica se o vetor $b$ pertence ao reticulado de $a_{1}, \ldots, a_{m}$, devolve a combinação linear não-negativa correspondente em caso positivo e devolve um vetor y tal que yb não é inteiro e ya $a_{i}$ é inteiro para todo $i$ em caso negativo.

Diante desses resultados, é natural nos perguntarmos se poderíamos obter resultados análogos no caso em que juntamos a restrição de não-negatividade dos cones com a restrição de integralidade dos reticulados. Chamamos de cone inteiro de $a_{1}, \ldots, a_{m}$ o conjunto dado por

$$
\text { int. cone }\left(a_{1}, \ldots, a_{m}\right):=\left\{a: a=\lambda_{1} a_{1}+\cdots+\lambda_{m} a_{m} \text {, onde } \lambda_{1}, \ldots, \lambda_{m} \in \mathbb{Z}_{\geq}\right\}
$$

ou seja, o conjunto de todos os vetores gerados por combinações inteiras não-negativas de $a_{1}, \ldots, a_{m}$.

Intuitivamente, podemos não apenas conjecturar resultados análogos aos teoremas mencionadas para o caso de cones inteiros, como também esperar que a teoria por trás de tais resultados esteja de alguma forma relacionada com a teoria de cones e reticulados. Por exemplo, podemos considerar

Conjectura 4.1. Sejam $a_{1}, \ldots, a_{m}, b$ vetores. O vetor $b$ pertence ao cone inteiro de $a_{1}, \ldots, a_{m}$ se $e$ somente se $b$ pertence ao cone e ao reticulado desses vetores.

Essa conjectura é falsa. Embora seja verdade que

$$
\text { int. cone }\left(a_{1}, \ldots, a_{m}\right) \subseteq \operatorname{cone}\left(a_{1}, \ldots, a_{m}\right) \cap \operatorname{ret}\left(a_{1}, \ldots, a_{m}\right)
$$

por definição, a inclusão inversa nem sempre é válida. Considere, por exemplo, os inteiros 2 e 3 . Temos que 1 está tanto no cone como no reticulado gerado por esses inteiros. No entanto, não há como gerar 1 através de uma combinação inteira não-negativa de 2 e 3 .

Em relação a complexidade, também podemos verificar um resultado desencorajador:

Teorema 4.2. Dados vetores $a_{1}, \ldots, a_{m}, b$, o problema de decidir se o vetor $b$ pertence ao cone inteiro de $a_{1}, \ldots, a_{m}$ é NP-completo.

Demonstração. Para demonstrar a NP-completude desse problema, vamos reduzir o problema da partição a ele. Sejam $\alpha_{1}, \ldots, \alpha_{n}$ os inteiros dados como entrada para o problema da partição, ou seja, sendo $\sigma:=\sum_{i=1}^{n} \alpha_{i}$, queremos decidir se existe um subconjunto de $\left\{\alpha_{1}, \ldots, \alpha_{n}\right\}$ tal que a soma de todos os elementos desse subconjunto é igual a $\sigma / 2$.

Considere então uma matriz $A \in \mathbb{R}^{(n+1) \times 2 n}$ e um vetor $b \in \mathbb{R}^{n+1}$ construídos da seguinte maneira:

$$
A:=\left[\begin{array}{cc}
a & 0 \\
I & I
\end{array}\right] ; \quad b:=\left(\begin{array}{c}
\sigma / 2 \\
1
\end{array}\right)
$$

Podemos então observar que, para qualquer $x:=\left(\xi_{1}, \ldots, \xi_{2 n}\right)$ inteiro e não-negativo vale que

$$
A x=\left[\begin{array}{cc}
a & 0 \\
I & I
\end{array}\right]\left(\begin{array}{c}
\xi_{1} \\
\vdots \\
\xi_{2 n}
\end{array}\right)=\left(\begin{array}{c}
\xi_{1} \alpha_{1}+\cdots+\xi_{n} \alpha_{n} \\
\xi_{1}+\xi_{n+1} \\
\vdots \\
\xi_{n}+\xi_{2 n}
\end{array}\right)=\left(\begin{array}{c}
\sigma / 2 \\
1 \\
\vdots \\
1
\end{array}\right)=b
$$

se e somente se $\xi_{1} \alpha_{1}+\cdots+\xi_{n} \alpha_{n}=\sigma / 2$ e $\xi_{i} \in\{0,1\}$ para todo $i=1, \ldots, n$. Mas nesse caso temos que o conjunto $\left\{\alpha_{i}: \xi_{i}=1\right\}$ é um subconjunto de $\left\{\alpha_{1}, \ldots, \alpha_{n}\right\}$ cuja soma é igual a $\sigma / 2$. Logo, o problema original foi reduzido a decidir se existe um vetor inteiro não-negativo $x$ tal que $A x=b$, ou seja, se $b$ está no cone inteiro das colunas de $A$. 
Assim, infelizmente não temos resultados análogos aos de cones e reticulados para cones inteiros. Entretanto, veremos a seguir um tipo especial de cone inteiro para o qual podemos obter resultados mais encorajadores.

Para cones inteiros em geral ainda podemos fazer algumas observações. Em especial, é importante mencionar que a união da restrição de não-negatividade com a de integralidade nos permite tratar combinações lineares sob um ponto de vista mais combinatório.

Por exemplo, sabendo que todo coeficiente positivo de uma combinação inteira não-negativa é pelo menos 1 , podemos verificar que a soma dos coeficientes é um limitante superior para o número de coeficientes positivos. Disso seguem algumas conseqüências simples mas significativas, como

Lema 4.3. Sejam $a_{1}, \ldots, a_{m}$ vetores e seja $v$ um vetor não-nulo. Suponha que $v=\sum_{i=1}^{m} \alpha_{i} a_{i}$, onde $\alpha_{1}, \ldots, \alpha_{m}$ são inteiros não-negativos. Se $v \notin\left\{a_{1}, \ldots, a_{m}\right\}$, então $\sum_{i=1}^{m} \alpha_{i} \geq 2$.

Demonstração. Como $v$ é não-nulo, $\sum_{i=1}^{m} \alpha_{i}>0$. Como $\alpha_{1}, \ldots, \alpha_{m}$ são inteiros não-negativos, precisamos apenas provar que $\sum_{i=1}^{m} \alpha_{i} \neq 1$. Mas isso é verdade, pois caso contrário teríamos $\alpha_{j}=1$ para algum $j$ e $\alpha_{i}=0$ para todo $i \neq j$, o que implicaria $v \in\left\{\alpha_{1}, \ldots, \alpha_{m}\right\}$.

Lema 4.4. Sejam $a_{1}, \ldots, a_{m}$ vetores e seja $v$ um vetor não-nulo. Suponha que $v=\sum_{i=1}^{m} \alpha_{i} a_{i}$, onde $\alpha_{1}, \ldots, \alpha_{m}$ são inteiros não-negativos. Se $v \notin\left\{a_{1}, \ldots, a_{m}\right\}$, então $v=v_{1}+v_{2}$, onde $v_{1}, v_{2}$ são vetores não-nulos em int. cone $\left(a_{1}, \ldots, a_{m}\right)$.

Demonstração. Sabemos pelo lema 4.3 que $\sum_{i=1}^{m} \alpha_{i} \geq 2$. Logo, o número $k$ de coeficientes não-nulos dentre $\alpha_{1}, \ldots, \alpha_{m}$ é maior do que 1. Sem perda de generalidade, vamos supor $\alpha_{1}, \ldots, \alpha_{k}>0$. Defina então vetores $v_{1}, v_{2}$ tais que $v_{1}:=\alpha_{1} a_{1}$ e $v_{2}:=\left(\alpha_{1}-1\right) a_{1}+\alpha_{2} a_{2}+\cdots+\alpha_{k} a_{k}$.

\subsection{Sistemas geradores de Hilbert}

Como vimos anteriormente, para qualquer conjunto finito $\mathcal{H}$ de vetores, temos que

$$
\text { int. cone }(\mathcal{H}) \subseteq \operatorname{cone}(\mathcal{H}) \cap \operatorname{ret}(\mathcal{H})
$$

e o que gostaríamos que valesse, mas infelizmente não vale para qualquer $\mathcal{H}$, é a inclusão inversa

$$
\text { int. cone }(\mathcal{H}) \supseteq \operatorname{cone}(\mathcal{H}) \cap \operatorname{ret}(\mathcal{H}) \text {. }
$$

Vamos então considerar apenas conjuntos que satisfazem a propriedade desejada. Dizemos que um conjunto finito $\mathcal{H}$ de vetores é um sistema gerador de Hilbert se

$$
\text { int. } \operatorname{cone}(\mathcal{H})=\operatorname{cone}(\mathcal{H}) \cap \operatorname{ret}(\mathcal{H}) \text {. }
$$

Para sistemas geradores de Hilbert, podemos unir os teoremas 2.22 e 3.15 para obter um teorema de alternativas para essa classe de cones inteiros.

Teorema 4.5. Sejam $h_{1}, \ldots, h_{m}, b$ vetores. Suponha que $h_{1}, \ldots, h_{m}$ formam um sistema gerador de Hilbert. $O$ vetor $b$ pertence ao cone inteiro de $h_{1}, \ldots, h_{m}$ se e somente se

(i) não existe um vetor y tal que $y b<0$ e $y h_{i} \geq 0$ para todo $i$;

(ii) não existe um vetor y tal que yb não é inteiro e yhi é inteiro para todo $i$. 
Demonstração. Segue da definição de sistema gerador de Hilbert e dos teoremas 2.22 e 3.15.

Apesar de termos resultados melhores para sistemas geradores de Hilbert do que para cones inteiros genéricos, é natural nos perguntarmos se a restrição imposta pela definição de sistemas geradores não é excessiva, reduzindo talvez demais o conjunto de cones e reticulados sobre os quais podemos trabalhar. Vamos verificar a seguir que, para qualquer conjunto de vetores, podemos construir um sistema gerador de Hilbert que gera o mesmo cone e o mesmo reticulado que o conjunto dado.

A idéia por trás da construção é a seguinte: se $a$ está no cone e no reticulado de um conjunto mas não está no cone inteiro, então em toda combinação não-negativa que gera $a$ há coeficientes fracionários. Combinações com coeficientes fracionários merecem, portanto, atenção especial como sendo o "obstáculo" que impede um dado conjunto de vetores de ser sistema gerador. Vamos dar um nome a essas combinações: o paralelepípedo gerado por $a_{1}, \ldots, a_{m}$ é o conjunto

$$
\operatorname{par}\left(a_{1}, \ldots, a_{m}\right)=\left\{\lambda_{1} a_{1}+\cdots+\lambda_{m} a_{m}: 0 \leq \lambda_{1}, \ldots, \lambda_{m}<1\right\} \text {. }
$$

Geometricamente, um paralelepípedo pode ser interpretado como a "caixa" cujas arestas são definidas pelos vetores, daí seu nome. A figura 4.1 mostra um exemplo desse conceito.

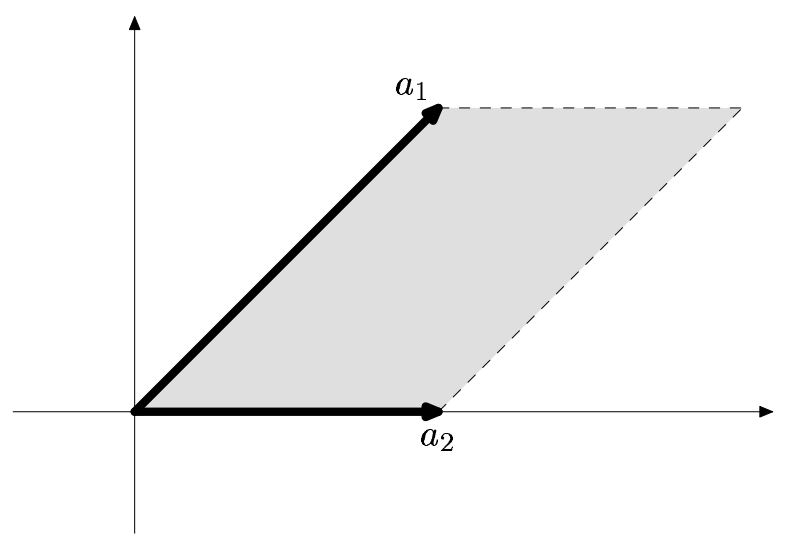

Figura 4.1: paralelepípedo gerado por $a_{1}, a_{2}$.

Diante dessa interpretação, podemos verificar que todo paralelepípedo é um conjunto limitado. Formalizando a idéia que acabamos de apresentar, o teorema abaixo mostra que, ao "completarmos" um dado conjunto de vetores com os vetores de seu reticulado que estão no paralelepípedo, podemos transformá-lo em sistema gerador de Hilbert que gera o mesmo cone e reticulado.

O teorema a seguir é devido a GoRDAN [1873]:

Teorema 4.6. Seja $\mathcal{A}$ um conjunto finito de vetores e seja $\mathcal{H}:=\mathcal{A} \cup(\operatorname{par}(\mathcal{A}) \cap \operatorname{ret}(\mathcal{A}))$. O conjunto $\mathcal{H}$ é sistema gerador de Hilbert satisfazendo cone $(\mathcal{H})=\operatorname{cone}(\mathcal{A}) e \operatorname{ret}(\mathcal{H})=\operatorname{ret}(\mathcal{A})$.

Demonstração. Temos $\operatorname{ret}(\mathcal{H})=\operatorname{ret}(\mathcal{A})$, pois o reticulado gerado é preservado se adicionarmos ao conjunto gerador um vetor já pertencente ao reticulado. Pelo mesmo argumento, podemos verificar que cone $(\mathcal{H})=\operatorname{cone}(\mathcal{A})$, visto que $\operatorname{par}(\mathcal{A}) \subseteq \operatorname{cone}(\mathcal{A})$. Resta provar que $\mathcal{H}$ é sistema gerador de Hilbert. Suponha então que $v$ é um vetor em $\operatorname{cone}(\mathcal{H}) \cap \operatorname{ret}(\mathcal{H})=\operatorname{cone}(\mathcal{A}) \cap \operatorname{ret}(\mathcal{A})$.

Estando $v$ em cone $(\mathcal{A})$, existem $\lambda_{1}, \ldots, \lambda_{m} \geq 0$ tais que $\lambda_{1} a_{1}+\cdots+\lambda_{m} a_{m}=v$. Vamos então considerar separadamente as partes inteira e fracionária dos coeficientes. Vamos denotar por $\{\alpha\}$ a 
parte fracionária de um número $\alpha$. Mais formalmente, $\{\alpha\}:=\alpha-\lfloor\alpha\rfloor$. Então:

$$
\begin{aligned}
v & =\lambda_{1} a_{1}+\cdots+\lambda_{m} a_{m} \\
& =\left(\left\lfloor\lambda_{1}\right\rfloor+\left\{\lambda_{1}\right\}\right) a_{1}+\cdots+\left(\left\lfloor\lambda_{m}\right\rfloor+\left\{\lambda_{m}\right\}\right) a_{m} \\
& =\left(\left\lfloor\lambda_{1}\right\rfloor a_{1}+\cdots+\left\lfloor\lambda_{m}\right\rfloor a_{m}\right)+\left(\left\{\lambda_{1}\right\} a_{1}+\cdots\left\{\lambda_{m}\right\} a_{m}\right) .
\end{aligned}
$$

Mais especificamente, podemos decompor o vetor $v$ como uma combinação inteira não-negativa:

$$
v=\left\lfloor\lambda_{1}\right\rfloor a_{1}+\cdots+\left\lfloor\lambda_{k}\right\rfloor a_{m}+1\left(\sum_{i=1}^{m}\left\{\lambda_{i}\right\} a_{i}\right) .
$$

Portanto, $v$ é gerado pela combinação inteira não-negativa de vetores em $\mathcal{A}$ mais um vetor em $\operatorname{par}(\mathcal{A})$. Mas esse vetor também está em $\operatorname{ret}(\mathcal{A})$, pois $v \in \operatorname{ret}(\mathcal{A})$ e, pela igualdade anterior,

$$
\sum_{i=1}^{m}\left\{\lambda_{i}\right\} a_{i}=v-\left\lfloor\lambda_{1}\right\rfloor a_{1}-\cdots-\left\lfloor\lambda_{k}\right\rfloor a_{m} .
$$

Portanto, $v \in$ int. cone $(\mathcal{H})$, o que prova que $\mathcal{H}$ é sistema gerador de Hilbert.

Temos garantida a existência de um sistema gerador de Hilbert para quaisquer cones e reticulados.

\subsection{Bases de Hilbert}

Como vimos na seção anterior, para qualquer conjunto $\mathcal{A}$ de vetores, $\mathcal{A} \cup(\operatorname{par}(\mathcal{A}) \cap \operatorname{ret}(\mathcal{A}))$ é um sistema gerador de Hilbert que gera $\operatorname{cone}(\mathcal{A})$ e $\operatorname{ret}(\mathcal{A})$. Entretanto, não é difícil construir exemplos nos quais tal conjunto contém muito mais vetores do que o necessário para conseguirmos essa propriedade.

Considere, por exemplo, um inteiro não-negativo $\alpha$. Como $(\alpha+1)-\alpha=1$, o reticulado gerado pelo conjunto $\mathcal{A}:=\{\alpha, \alpha+1\}$ é $\mathbb{Z}$. Ademais, $\operatorname{cone}(\mathcal{A})=\mathbb{Z}_{\geq}$e $\mathcal{A} \cup(\operatorname{par}(\mathcal{A}) \cap \operatorname{ret}(\mathcal{A}))$ corresponde aos inteiros no intervalo $[0,2 \alpha+1)$. Mas para gerar $\mathbb{Z}_{\geq}$precisamos apenas do inteiro 1 , ou seja, nesse exemplo temos um sistema arbitrariamente maior do que o necessário.

Vamos então dirigir nosso estudo aos "menores" sistemas geradores possíveis relativos a dado cone e reticulado. Dizemos que um sistema gerador de Hilbert $\mathcal{H}$ é uma base de Hilbert se $\mathcal{H}$ é minimal em relação ao seu cone ou ao seu reticulado. Mais especificamente, $\mathcal{H}$ é uma base de Hilbert se, para todo subconjunto próprio $\mathcal{H}^{\prime}$ de $\mathcal{H}$, vale pelo menos uma dentre as seguintes propriedades:

(i) int. cone $\left(\mathcal{H}^{\prime}\right) \neq \operatorname{cone}\left(\mathcal{H}^{\prime}\right) \cap \operatorname{ret}\left(\mathcal{H}^{\prime}\right)$;

(ii) $\operatorname{cone}\left(\mathcal{H}^{\prime}\right) \neq \operatorname{cone}(\mathcal{H})$;

(iii) $\operatorname{ret}\left(\mathcal{H}^{\prime}\right) \neq \operatorname{ret}(\mathcal{H})$.

Vamos então verificar como podemos obter uma base de Hilbert que gera $\operatorname{cone}(\mathcal{A})$ e $\operatorname{ret}(\mathcal{A})$. Queremos um subconjunto minimal de $\operatorname{cone}(\mathcal{A}) \cap \operatorname{ret}(\mathcal{A})$ que gere o mesmo cone e reticulado e ao mesmo tempo seja um sistema gerador de Hilbert.

Baseando-se no lema 4.4, podemos considerar a seguinte idéia intuitiva: se um vetor não-nulo em cone $(\mathcal{A}) \cap \operatorname{ret}(\mathcal{A})$ é a soma de dois vetores também não-nulos em $\operatorname{cone}(\mathcal{A}) \cap \operatorname{ret}(\mathcal{A})$, é razoável esperar que esse vetor $n \tilde{a} o$ faça parte de uma base de Hilbert. Outra maneira de interpretar esse fato 
é: se queremos um gerador minimal, é razoável esperar que os elementos desse gerador não possam ser quebrados em elementos menores. Mais formalmente, vamos considerar

$$
\mathcal{H}:=\{g \in \mathcal{G}: g \text { não é soma de dois vetores em } \mathcal{G}\} \text {, onde } \mathcal{G}=\operatorname{cone}(\mathcal{A}) \cap \operatorname{ret}(\mathcal{A}) \backslash\{0\} \text {. }
$$

O resultado abaixo mostra o caráter minimal do conjunto assim definido:

Lema 4.7. Seja $\mathcal{A}$ um conjunto de vetores e seja $\mathcal{H}$ definido como em (4.1). Para todo sistema gerador de Hilbert $\mathcal{H}^{\prime}$ que satisfaz cone $\left(\mathcal{H}^{\prime}\right)=\operatorname{cone}(\mathcal{A})$ e $\operatorname{ret}\left(\mathcal{H}^{\prime}\right)=\operatorname{ret}(\mathcal{A})$, temos $\mathcal{H} \subseteq \mathcal{H}^{\prime}$.

Demonstração. Tome $h \in \mathcal{H}$. Sabemos que $h \in \operatorname{cone}(\mathcal{A}) \cap \operatorname{ret}(\mathcal{A})=\operatorname{cone}\left(\mathcal{H}^{\prime}\right) \cap \operatorname{ret}\left(\mathcal{H}^{\prime}\right)$ por hipótese. Sabemos também que $h$ é não-nulo. Logo, supondo que $h \notin \mathcal{H}^{\prime}$, sabemos pelo lema 4.4 que existem dois vetores $h_{1}, h_{2} \in \operatorname{cone}(\mathcal{A}) \cap \operatorname{ret}(\mathcal{A})$ tais que $h=h_{1}+h_{2}$. Isso é uma contradição.

Esse conjunto, no entanto, pode ser pequeno demais ou mesmo vazio. Considere o caso em que $\mathcal{A}=\{-1,1\}$. Temos nesse caso que $\operatorname{cone}(\mathcal{A}) \cap \operatorname{ret}(\mathcal{A})=\mathbb{Z}$ e sabemos que qualquer inteiro não-nulo pode ser escrito como soma de dois inteiros não-nulos. Por outro lado, ele ainda pode servir a nossos propósitos se considerarmos o caso particular de cones pontudos.

Teorema 4.8. Seja $\mathcal{A}$ um conjunto de vetores e seja $\mathcal{H}$ definido como em (4.1). Se cone $(\mathcal{A})$ é pontudo, então $\mathcal{H}$ é uma base de Hilbert tal que cone $(\mathcal{H})=\operatorname{cone}(\mathcal{A})$ e $\operatorname{ret}(\mathcal{H})=\operatorname{ret}(\mathcal{A})$.

Demonstração. Pelo teorema 4.6 e pelo lema 4.7, sabemos que $\mathcal{H}$ é um conjunto finito e contido em qualquer sistema gerador de Hilbert que gera o mesmo cone e reticulado que $\mathcal{A}$. Logo, precisamos apenas provar que o próprio $\mathcal{H}$ também é um sistema gerador de Hilbert dessa forma.

Primeiramente, vamos demonstrar que $\operatorname{cone}(\mathcal{H})=\operatorname{cone}(\mathcal{A})$ e $\operatorname{ret}(\mathcal{H})=\operatorname{ret}(\mathcal{A})$. Para isso, basta provar que $\mathcal{A} \subseteq$ cone $(\mathcal{H}) \cap \operatorname{ret}(\mathcal{H})$. Na verdade, para obter essa propriedade vamos provar algo ainda mais forte: que $\mathcal{A} \cup(\operatorname{par}(\mathcal{A}) \cap \operatorname{ret}(\mathcal{A})) \subseteq \operatorname{cone}(\mathcal{H}) \cap \operatorname{ret}(\mathcal{H})$.

Como cone $(\mathcal{A})$ é pontudo, existe $b$ tal que $b a>0$ para qualquer $a \neq 0$ em $\operatorname{cone}(\mathcal{A})$ : podemos tomar por exemplo um vetor $b$ que define um hiperplano suporte do cone. Vamos então supor por contradição que existe $a \neq 0 \mathrm{em} \mathcal{A} \cup(\operatorname{par}(\mathcal{A}) \cap \operatorname{ret}(\mathcal{A}))$ que não está em cone $(\mathcal{H}) \cap \operatorname{ret}(\mathcal{H})$ e que tal $a$ tem valor mínimo de $b a$. Como $\mathcal{A} \cup(\operatorname{par}(\mathcal{A}) \cap \operatorname{ret}(\mathcal{A}))$ é um conjunto finito, o mínimo existe. Mas pela definição de $\mathcal{H}$ existem $h_{1}, h_{2} \in \operatorname{cone}(\mathcal{A}) \cap \operatorname{ret}(\mathcal{A}) \backslash\{0\}$ tais que $a=h_{1}+h_{2}$.

Por outro lado, vimos na demonstração do teorema 4.6 que $h_{1}, h_{2}$ podem ambos ser escritos como combinação inteira não-negativa de vetores em $\mathcal{A} \cup(\operatorname{par}(\mathcal{A}) \cap \operatorname{ret}(\mathcal{A}))$. Disso segue que ba é uma combinação inteira não-negativa de produtos internos de $b$ com os vetores $\operatorname{de} \mathcal{A} \cup(\operatorname{par}(\mathcal{A}) \cap \operatorname{ret}(\mathcal{A}))$. Como isso é uma contradição com a minimalidade de $b a$, estamos feitos.

Resta demonstrar que $\mathcal{H}$ é um sistema gerador de Hilbert. Suponha então que existe um vetor $h$ tal que $h \in \operatorname{cone}(\mathcal{H}) \cap \operatorname{ret}(\mathcal{H})=\operatorname{cone}(\mathcal{A}) \cap \operatorname{ret}(\mathcal{A})$ e $h \notin$ int. cone $(\mathcal{H})$. Vamos supor que $h$ é escolhido de forma que $b h$ seja mínimo. Então $h \in \mathcal{A} \cup(\operatorname{par}(\mathcal{A}) \cap \operatorname{ret}(\mathcal{A}))$, pois caso contrário conseguiríamos obter um vetor $h^{\prime}$ com as mesmas propriedades de $h$ mas tal que $b h^{\prime}<b h$. Por outro lado, sabemos pelo lema 4.4 que $h=h_{1}+h_{2}$, onde $h_{1}, h_{2}$ são vetores não-nulos em cone $(\mathcal{A}) \cap \operatorname{ret}(\mathcal{A})$, contradição.

Juntando esse teorema com o lema 4.7, podemos concluir que

Teorema 4.9. Seja $\mathcal{A}$ um conjunto de vetores. Se cone $(\mathcal{A})$ é pontudo, então existe uma única base de Hilbert $\mathcal{H}$ tal que cone $(\mathcal{H})=\operatorname{cone}(\mathcal{A})$ e $\operatorname{ret}(\mathcal{H})=\operatorname{ret}(\mathcal{A})$.

Essa demonstração de unicidade é devido a VAN DER Corput [1931] e SchriJver [1986]. 


\subsection{Algoritmo de Chandrasekaran}

Como vimos anteriormente, para sistemas geradores de Hilbert podemos definir um teorema de alternativas para caracterizar a pertinência ao cone inteiro. No entanto, até agora ainda não foi mencionado como obter a combinação inteira não-negativa que certifica essa pertinência.

Vamos apresentar um algoritmo polinomial que, dado um sistema gerador de Hilbert $\mathcal{H}$ e um vetor $v \in \operatorname{int}$. cone $(\mathcal{H})$, devolve uma combinação inteira não-negativa dos vetores em $\mathcal{H}$ que gera $h$. Primeiramente, podemos observar que a existência de inteiros não-negativos $\lambda_{1}, \ldots, \lambda_{m}$ tais que

$$
\lambda_{1} h_{1}+\cdots+\lambda_{m} h_{m}=v
$$

pode ser reformulada em termos de subtrações que reduzem $v$ a zero:

$$
v-\lambda_{1} h_{1}-\cdots-\lambda_{m} h_{m}=0 .
$$

Isso nos permite pensar em uma primeira abordagem simples para o cálculo de $\lambda_{1}, \ldots, \lambda_{m}$ : utilizar um método guloso para subtrair de $v$ o máximo de vezes possível cada vetor em $\mathcal{H}$. Considere o vetor $h_{1} \in \mathcal{H}$ e suponha que $h_{1}$ não está na face minimal de cone $(\mathcal{H})$, ou seja, $M h_{1} \neq 0$, onde $M$ é uma matriz tal que $\operatorname{cone}(\mathcal{H})=\{x: M x \leq 0\}$. O fato de $M h_{1}$ não ser nulo implica que o valor de

$$
\sigma_{1}:=\max \left\{\sigma: v-\sigma h_{1} \in \operatorname{cone}(\mathcal{H})\right\}
$$

é finito. Vamos então supor sem perda de generalidade que $h_{1}, \ldots, h_{k}, k \leq m$ são os vetores de $\mathcal{H}$ que não estão na face minimal do cone gerado e definir a seguinte seqüência de vetores:

$$
\begin{aligned}
& v_{0}:=v \\
& v_{i}:=v_{i-1}-\left\lfloor\sigma_{i}\right\rfloor h_{i}, \text { onde } \sigma_{i} \text { é o maior racional tal que } v_{i-1}-\sigma_{i} h_{i} \in \operatorname{cone}(\mathcal{H}) .
\end{aligned}
$$

Seja $H$ a matriz cujas colunas são os vetores em $\mathcal{H}$. Dizer que $v_{i-1}-\sigma_{i} h_{i} \in \operatorname{cone}(\mathcal{H})$ equivale a dizer que existe $x \geq 0$ tal que $H x+\sigma_{i} h_{i}=v_{i-1}$. Logo, $\sigma_{i}$ é o valor ótimo do programa linear

$$
\max \left\{\left(\begin{array}{ll}
0 & 1
\end{array}\right)\left(\begin{array}{c}
x \\
\sigma
\end{array}\right):\left[A h_{i}\right]\left(\begin{array}{c}
x \\
\sigma
\end{array}\right)=v_{i-1} ; x \geq 0 ; \sigma \geq 0\right\}
$$

e, portanto, cada $\sigma_{i}$ pode ser calculado em tempo polinomial.

Vamos agora considerar $v_{k}$, o último vetor dessa seqüência. Por construção, sabemos que $v_{k}$ é um vetor em cone $(\mathcal{H}) \cap \operatorname{ret}(\mathcal{H})$. Logo, como $\mathcal{H}$ é um sistema gerador de Hilbert, $v_{k} \in$ int. cone $(\mathcal{H})$ :

$$
v_{k}=\lambda_{1} h_{1}+\cdots+\lambda_{k} h_{k}+\lambda_{k+1} h_{k+1}+\cdots+\lambda_{m} h_{m}
$$

onde $\lambda_{1}, \ldots, \lambda_{m}$ são inteiros não-negativos. Mas note que, também por construção, podemos verificar que $\lambda_{1}, \ldots, \lambda_{k}=0$, pois o contrário violaria a maximalidade de algum $\sigma_{i}$. Concluindo, temos que

$$
v=\left\lfloor\sigma_{1}\right\rfloor h_{1}+\cdots+\left\lfloor\sigma_{k}\right\rfloor h_{k}+c_{k},
$$

onde $c_{k} \in$ int. cone $\left(h_{k+1}, \ldots, h_{m}\right)$. Em outras palavras, o nosso problema inicial foi reduzido ao caso em que o vetor está na face minimal do cone. Vamos então trabalhar sobre esse caso.

Vamos denotar por $\mathcal{C}$ o cone gerado por $h_{k+1}, \ldots, h_{m}$, ou seja, precisamente a face minimal do cone original. Por estarmos considerando agora um subespaço linear, temos sobre os vetores uma propriedade que não tínhamos no caso geral: para todo $h_{i} \operatorname{com} k+1 \leq i \leq m$ temos $-h_{i} \in \mathcal{C}$ :

$$
\lambda_{k+1} h_{k+1}+\cdots+\lambda_{i} h_{i}+\cdots+\lambda_{m} h_{m}=-h_{i}
$$


Podemos reformular essa igualdade como uma combinação que gera o vetor nulo:

$$
\lambda_{k+1} h_{k+1}+\cdots+\left(1+\lambda_{i}\right) h_{i}+\cdots+\lambda_{m} h_{m}=0 .
$$

Uma propriedade dessa combinação que merece atenção especial é o fato de que, como $\lambda_{i} \geq 0$, temos que o coeficiente relativo a $h_{i}$ é estritamente positivo. Ademais, pela escolha arbitrária de $i$ podemos obter uma combinação análoga para todo $i$. Somando todas essas combinações, temos

$$
\lambda_{k+1}^{\prime} h_{k+1}+\cdots+\lambda_{m}^{\prime} h_{m}=0
$$

onde $\lambda_{k+1}^{\prime}, \ldots, \lambda_{m}^{\prime}>0$. Sem perda de generalidade, podemos supor que $\lambda_{k+1}^{\prime}, \ldots, \lambda_{m}^{\prime}$ também são inteiros, pois se isso não for verdade podemos multiplicá-los por um inteiro adequado.

O valor dessa combinação inteira positiva está no fato de que ela gera o vetor nulo e, portanto, pode ser somada a uma dada combinação sem efeito no resultado. Escolhendo essa combinação como sendo, digamos, uma combinação inteira que gera $c_{k}$, isso significa que podemos somar a combinação positiva até obter uma combinação inteira não-negativa! E tudo isso em tempo polinomial.

Teorema 4.10. Existe um algoritmo polinomial para, dados vetores $h_{1}, \ldots, h_{m}$, b tais que $h_{1}, \ldots, h_{m}$ formam um sistema gerador de Hilbert, verifica se o vetor b pertence ao cone inteiro de $h_{1}, \ldots, h_{m}$, devolve a combinação inteira não-negativa correspondente em caso positivo e devolve um vetor y tal que $y b<0$ e $y h_{i} \geq 0$ para todo $i$ ou um vetor y tal que yb não é inteiro e yh $h_{i}$ é inteiro para todo $i$ em caso negativo.

A beleza desse algoritmo, demonstrado por Chandrasekaran [1981], é o fato de resolver o problema através da combinação de um método guloso simples com a aplicação direta dos algoritmos para obter as combinações não-negativas e combinações inteiras, tirando proveito dos resultados obtidos anteriormente.

\subsection{Bases isomorfas e imersões em reticulados}

Seja $\mathcal{H}:=\left\{h_{1}, \ldots, h_{m}\right\}$ uma base de Hilbert em $\mathbb{R}^{n}$ e seja $\varphi$ uma função de $\mathbb{R}^{n}$ em $\mathbb{R}^{r}$. Considere o conjunto

$$
\varphi(\mathcal{H}):=\left\{\varphi\left(h_{1}\right), \ldots, \varphi\left(h_{m}\right)\right\} .
$$

Vamos supor ainda que $\varphi$ é uma função bijetora satisfazendo linearidade:

$$
\varphi\left(\lambda_{1} a_{1}+\lambda_{2} a_{2}\right)=\lambda_{1} \varphi\left(a_{1}\right)+\lambda_{2} \varphi\left(a_{2}\right) \text {, para quaisquer } \lambda_{1}, \lambda_{2} \in \mathbb{R} \text { e } a_{1}, a_{2} \in \mathbb{R}^{n} .
$$

Em termos de combinações lineares dos vetores em $\mathcal{H}$, essa linearidade implica que

$$
\varphi\left(\lambda_{1} h_{1}+\cdots+\lambda_{m} h_{m}\right)=\lambda_{1} \varphi\left(h_{1}\right)+\cdots+\lambda_{m} \varphi\left(h_{m}\right) \text { para quaisquer } \lambda_{1}, \ldots, \lambda_{m} \in \mathbb{R} \text {. }
$$

Podemos verificar, portanto, que $\varphi(\mathcal{H})$ também é uma base de Hilbert, pois

$$
\begin{array}{rll}
h \in \operatorname{cone}(\mathcal{H}) & \text { se e somente se } & \varphi(h) \in \operatorname{cone}(\varphi(\mathcal{H})) \\
h \in \operatorname{ret}(\mathcal{H}) & \text { se e somente se } & \varphi(h) \in \operatorname{ret}(\varphi(\mathcal{H})) \\
h \in \text { int. cone }(\mathcal{H}) & \text { se e somente se } & \varphi(h) \in \operatorname{int} \operatorname{cone}(\varphi(\mathcal{H}))
\end{array}
$$

Dizemos nesse caso que esse conjunto é uma base isomorfa a $\mathcal{H}$. 
Teorema 4.11. Seja $\mathcal{H}$ uma base de Hilbert em $\mathbb{R}^{n}$ e seja $r:=\operatorname{dim}(\mathcal{H})$. Existe uma base de Hilbert $\mathcal{H}^{\prime}$ em $\mathbb{R}^{n}$ tal que $\operatorname{ret}\left(\mathcal{H}^{\prime}\right)=\mathbb{Z}^{r}$ e $\mathcal{H}^{\prime}$ é isomorfa a $\mathcal{H}$.

Demonstração. Seja $R \in \mathbb{R}^{n \times r}$ uma matriz cujo conjunto de colunas é uma base do reticulado gerado por $\mathcal{H}$. Vamos definir uma função $\varphi: \mathbb{R}^{n} \rightarrow \mathbb{R}^{r}$ da seguinte forma:

$$
\varphi(h)=x \text {, onde } x \text { é a solução de } R x=h .
$$

Como as colunas de $R$ são linearmente independentes, temos que $\varphi$ é uma função bijetora e que

$$
\varphi^{-1}(x)=R x .
$$

Ademais, para quaisquer $\lambda_{1}, \lambda_{2} \in \mathbb{R}$ e $a_{1}, a_{2} \in \mathbb{R}^{n}$, temos que $\varphi\left(\lambda_{1} a_{1}+\lambda_{2} a_{2}\right)=\lambda_{1} \varphi\left(a_{1}\right)+\lambda_{2} \varphi\left(a_{2}\right)$, o que nos permite concluir que $\mathcal{H}$ e $\mathcal{H}^{\prime}:=\varphi(\mathcal{H})$ são bases isomorfas.

Se supormos que $h^{\prime}$ é um vetor em $\mathbb{Z}^{r}$, então $R h^{\prime} \in \operatorname{ret}(\mathcal{H})$. Logo, nesse caso temos $h^{\prime} \in \operatorname{ret}\left(\mathcal{H}^{\prime}\right)$. Por outro lado, se supormos que $h^{\prime} \in \operatorname{ret}\left(\mathcal{H}^{\prime}\right)$, então $R h^{\prime} \in \operatorname{ret}(\mathcal{H})$. Isso implica que $h^{\prime}$ é um vetor inteiro nesse caso, pois sabemos que as colunas da matriz $R$ são linearmente independentes.

Uma base de Hilbert definida como no teorema acima possui algumas propriedades úteis: ela gera todo o espaço, todos os seus vetores são inteiros e sua propriedade de sistema gerador de Hilbert pode ser formulada como

$$
\text { int. cone }\left(\mathcal{H}^{\prime}\right)=\operatorname{cone}\left(\mathcal{H}^{\prime}\right) \cap \mathbb{Z}^{r} \text {. }
$$

Ou seja, todo vetor no cone inteiro é um vetor inteiro no cone e vice-versa.

Ademais, substituindo a função inversa da demonstração por uma função definida pela base de um reticulado qualquer de mesma dimensão, podemos generalizar o teorema da seguinte forma:

Teorema 4.12. Seja $\mathcal{H}$ uma base de Hilbert e seja $r:=\operatorname{dim}(\mathcal{H})$. Para todo reticulado $\mathcal{R}$ tal que $\operatorname{dim}(\mathcal{R})=r$, existe uma base de Hilbert $\mathcal{H}^{\prime}$ tal que $\operatorname{ret}\left(\mathcal{H}^{\prime}\right)=\mathcal{R}$ e $\mathcal{H}^{\prime}$ é isomorfa a $\mathcal{H}$.

Demonstração. Basta substituir $R$ na demonstração anterior por uma base do reticulado $\mathcal{R}$.

Informalmente, os resultados anteriores implicam que uma base de Hilbert pode ser "imersa" em qualquer reticulado de mesma dimensão que a sua. Em particular, o reticulado $\mathbb{Z}^{r}$ é a imersão "canônica" que representa a ligação entre todas as bases de Hilbert de mesma dimensão.

\subsection{Bases totalmente integrais}

Antes de encerrar o capítulo, vamos estender as bases "especiais" que vimos na seção anterior para o caso em que os vetores não geram todo o espaço. Dizemos que um sistema gerador de Hilbert $\mathcal{H}$ em $\mathbb{Z}^{n}$ é totalmente integral se

$$
\operatorname{ret}(\mathcal{H})=\operatorname{lin}(\mathcal{H}) \cap \mathbb{Z}^{n}
$$

e essa definição é naturalmente estendida para bases de Hilbert. Para sistemas geradores de Hilbert desse tipo, podemos verificar que

$$
\text { int. cone }(\mathcal{H})=\operatorname{cone}(\mathcal{H}) \cap \mathbb{Z}^{n}
$$

ou seja, todo vetor inteiro no cone pertence ao cone inteiro.

Exemplos de sistemas geradores de Hilbert que são totalmente integrais são aqueles que formam matrizes totalmente unimodulares. Dizemos que uma matriz $H$ é totalmente unimodular se todo subdeterminante de $A$ é igual a 0,1 ou -1 . Segue diretamente da regra de Cramer que 
Lema 4.13. Sejam $h_{1}, \ldots, h_{m}$ vetores e seja $H$ a matriz cujas colunas são esses vetores. Se $H$ é totalmente unimodular, então $h_{1}, \ldots, h_{m}$ formam um sistema gerador de Hilbert totalmente integral.

Esse conceito é particularmente interessante porque esse tipo de matriz é facilmente extensível:

Lema 4.14. Se A é uma matriz totalmente unimodular e e é um vetor canônico, então [A e] é uma matriz totalmente unimodular.

É importante observar que o conceito de sistemas geradores de Hilbert e sistemas geradores de Hilbert totalmente integrais não são equivalentes. A figura 4.2 mostra um exemplo desse fato.

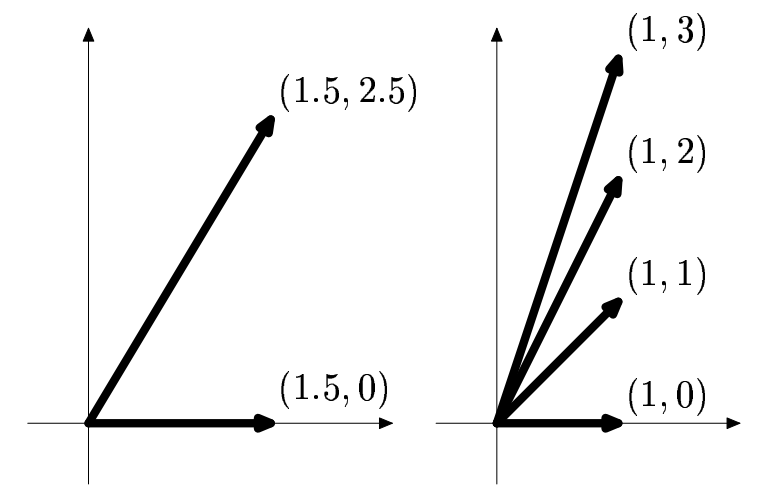

Figura 4.2: $\quad \grave{A}$ esquerda, um exemplo de sistema gerador de Hilbert que não é totalmente integral: o vetor $(1,1)$ é inteiro e pertence ao cone, mas não pertence ao cone inteiro. À direita, um exemplo de sistema gerador de Hilbert totalmente integral.

O que estamos chamando de sistemas geradores de Hilbert totalmente integrais são na verdade o que Giles e Pulleyblank [1979] definiram originalmente como bases de Hilbert. No capítulo a seguir, vamos estudar precisamente a motivação desses autores ao introduzirem esse conceito. 


\section{Capítulo 5}

\section{Programação inteira e total dual integralidade}

Neste capítulo, vamos considerar problemas de programação linear inteira sob o ponto de vista de sistemas totalmente dual integrais, que na verdade foram a motivação original para o conceito de bases de Hilbert. Vamos mostrar também alguns algoritmos de reconhecimento que são polinomiais sob a hipótese de que a dimensão considerada é fixa.

Convém mencionar que vamos enfatizar principalmente as relações entre bases de Hilbert e esses sistemas. Total dual integralidade é um tópico bastante rico por si próprio e existem muitos resultados interessantes na literatura a respeito desse assunto que não estão presentes neste trabalho.

\subsection{Programação inteira e poliedros inteiros}

Resolver um programa linear inteiro significa resolver um programa linear sob a restrição adicional de que a solução deve ser inteira. Tipicamente, denotaremos um programa inteiro por

$$
\max \{c x: A x \leq b ; x \text { inteiro }\}
$$

Se desconsiderarmos a restrição de integralidade, temos a relaxação linear desse programa. Para muitos programas inteiros, uma versão análoga do teorema da dualidade não vale: em geral,

$$
\max \{c x: A x \leq b ; x \text { inteiro }\}<\min \{y b: y A=c ; y \geq 0 ; y \text { inteiro }\} .
$$

Mas mesmo sem o teorema da dualidade, podemos demonstrar que resolver um programa inteiro é polinomialmente equivalente a resolver um sistema de desigualdades lineares diofantinas ou resolver um sistema de equações lineares diofantinas sob a restrição de que a solução é não-negativa. Logo, segue diretamente do teorema 4.2 que todos esses problemas são NP-difíceis.

Neste capítulo, vamos considerar principalmente o caso particular de programas lineares inteiros formulados sobre poliedros inteiros. Dizemos que um poliedro $\mathcal{P}$ é inteiro se toda face de $\mathcal{P}$ contém um vetor inteiro. O teorema a seguir mostra por que tais poliedros são particularmente interessantes.

Teorema 5.1. Existe um algoritmo polinomial que, dados uma matriz $A$ e vetores $b, c$ tais que o poliedro $\{x: A x \leq b\}$ é inteiro, resolve o programa inteiro $\max \{c x: A x \leq b ; x$ inteiro $\}$. 
Demonstração. Como mencionamos no capítulo 2, existe um algoritmo polinomial para resolver um programa linear. Portanto, se o dado programa admite solução, podemos obter em tempo polinomial o valor ótimo $\delta$ de sua relaxação linear e um vetor $x^{\prime}$, não necessariamente inteiro, do poliedro tal que $c x^{\prime}=\delta$. A partir de $c x^{\prime}$ podemos obter as restrições de $A x \leq b$ que determinam a face minimal à qual $x^{\prime}$ pertence. O problema então é reduzido a obter uma solução para um sistema linear diofantino e vimos no capítulo 3 um algoritmo para resolver sistemas diofantinos em tempo polinomial.

Assim, programas formulados sobre poliedros inteiros são tratáveis. Esse fato torna interessante obter caracterizações para poliedros inteiros. Vamos apresentar a seguir duas caracterizações que foram demonstradas por EDMONDS E GiLEs [1977].

A primeira caracterização utiliza o conceito de hiperplanos suporte. Dizemos que o conjunto

$$
\{x: c x=\delta\}
$$

é um hiperplano suporte do poliedro $\{x: A x \leq b\}$ se $\delta$ é o valor ótimo de $\max \{c x: A x \leq b\}$.

Teorema 5.2. Um poliedro é inteiro se e somente se todo hiperplano suporte desse poliedro contém vetores inteiros.

Demonstração. Seja $\mathcal{P}:=\{x: A x \leq b\}$. Como todo hiperplano suporte de $\mathcal{P}$ contém uma face de $\mathcal{P}$ por definição, então claramente temos que todo hiperplano suporte de $\mathcal{P}$ contém vetores inteiros se $\mathcal{P}$ é um poliedro inteiro. Resta demonstrar a recíproca.

Suponha que todo hiperplano suporte de $\mathcal{P}$ contém vetores inteiros. Podemos supor sem perda de generalidade que $A$ e $b$ são inteiros. Seja $\mathcal{F}$ uma face minimal de $\mathcal{P}$ e seja $A^{\prime} x \leq b^{\prime}$ um subsistema de $A x \leq b$ tal que $\mathcal{F}=\left\{x: A^{\prime} x=b^{\prime}\right\}$.

Se $\overline{\mathcal{F}}$ não contém vetores inteiros, então o sistema linear $A^{\prime} x=b^{\prime}$ não contém soluções inteiras. Isso implica pelo teorema 3.15 que existe $y$ tal que $c:=y A^{\prime}$ é inteiro e $\delta:=y b^{\prime}$ não é inteiro. Podemos supor sem perda de generalidade que $y>0$. Se isso não for verdade, podemos somar inteiros aos elementos de $y$ que não são positivos. Como $A$ e $b$ são inteiros, essa soma não afeta a propriedade de que $y A$ é inteiro e $y b$ não é inteiro.

Considere então o conjunto $\{x: c x=\delta\}$. Esse conjunto é hiperplano suporte de $\mathcal{P}$, pois $c x \leq \delta$ para todo $x \in \mathcal{P}$ e pelo menos um elemento de $\mathcal{P}$ satisfaz essa restrição com igualdade, visto que o conjunto $\mathcal{F}$, sendo uma face, não é vazio. Como $\delta$ não é um inteiro e $c$ é um vetor inteiro, a equação diofantina $c x=\delta$ não pode ter soluções inteiras. Logo, existe um hiperplano suporte $\mathcal{H}$ que não contém vetores inteiros. Isso é uma contradição com a hipótese.

A segunda caracterização pode ser diretamente demonstrada como sendo corolário da primeira.

Corolário 5.3. Seja $\mathcal{P}$ um poliedro. As duas afirmações a seguir são equivalentes:

(i) para todo vetor $c$, se o programa linear $\max \{c x: x \in \mathcal{P}\}$ admite solução, então esse programa admite solução inteira;

(ii) para todo vetor inteiro $c$, se o programa linear $\max \{c x: x \in \mathcal{P}\}$ admite solução, então o valor ótimo desse programa linear é inteiro.

Demonstração. Se $c$ é inteiro e $\max \{c x: A x \leq b\}$ admite uma solução ótima inteira, então o valor ótimo é claramente inteiro e um dos lados já está demonstrado. Suponha então que para todo vetor inteiro $c$ o valor ótimo de $\max \{c x: A x \leq b\}$ é inteiro se existir. Seja $\mathcal{H}=\{x: c x=\delta\}$ um 
hiperplano suporte do poliedro $\mathcal{P}:=\{x: A x \leq b\}$. Podemos supor que os elementos de $c$ são inteiros relativamente primos, pois multiplicar $c$ e $\delta$ por um mesmo valor preserva $\mathcal{H}$.

Como $c$ é inteiro, então $\delta:=\max \{c x: A x \leq b\}$ é inteiro por hipótese. Como os elementos de $c$ são relativamente primos, não pode existir $\lambda$ tal que $\lambda c$ é inteiro e $\lambda \delta$ não é inteiro. Logo, pelo teorema 3.15 , a equação diofantina $c x=\delta$ admite solução inteira e concluímos que todo hiperplano suporte de $\mathcal{P}$ contém um vetor inteiro. O resultado desejado segue, portanto, do teorema 5.2.

Na seção a seguir, vamos mostrar sistemas que definem poliedros inteiros sob certas hipóteses.

\subsection{Sistemas totalmente dual integrais}

O conceito de total dual integralidade foi apresentado por Edmonds E Giles [1977]. Seja $A$ uma matriz inteira e seja $b$ um vetor. Dizemos que $A x \leq b$ é um sistema totalmente dual integral se, para todo $c$ inteiro, existe uma solução inteira para

$$
\min \{y b: y A=c ; y \geq 0\}
$$

supondo que esse programa admite solução. Pelas caracterizações vistas na seção anterior, temos:

Teorema 5.4. Seja A uma matriz inteira e seja b um vetor. Suponha que o sistema $A x \leq b$ é totalmente dual integral. Se b é inteiro, então o poliedro $\{x: A x \leq b\}$ é inteiro.

Demonstração. Se $A x \leq b$ é um sistema totalmente dual integral, então o valor ótimo do programa linear $\min \{y b: y \geq 0, y A=c\}$ é obtido por um $y$ inteiro sempre que $c$ for inteiro e o programa admitir solução. Com a hipótese adicional de que $b$ é inteiro, podemos concluir que o valor ótimo é inteiro sempre que $c$ for inteiro e o programa admitir solução. Temos então a afirmação (ii) do corolário 5.3. A integralidade do poliedro segue, portanto, da afirmação (i) por equivalência.

Como conseqüência, se $A x \leq b$ é totalmente dual integral, então para quaisquer $b, c$ inteiros,

$$
\max \{c x: A x \leq b ; x \text { inteiro }\}=\min \{y b: y A=c ; y \geq 0 ; y \text { inteiro }\}
$$

se ambos os problemas admitem solução. Pode-se dizer então que os sistemas totalmente dual integrais representam problemas tratáveis em programação inteira, da mesma forma como os sistemas geradores de Hilbert representam cones inteiros tratáveis.

Os resultados a seguir, de Giles E Pulleyblank [1979] e Schrijver [1981], mostram que a relação entre os dois conceitos é, na verdade, muito mais profunda do que essa analogia simples:

Teorema 5.5. Seja A uma matriz inteira e seja b um vetor. O sistema $A x \leq b$ é totalmente dual integral se e somente se, para toda face $\mathcal{F}$ do poliedro $\mathcal{P}:=\{x: A x \leq b\}$, o conjunto das linhas ativas de $A$ em $\mathcal{F}$ é um sistema gerador de Hilbert totalmente integral.

Demonstração. Primeiramente, vamos supor que $A x \leq b$ é totalmente dual integral. Seja $\mathcal{F}$ uma face de $\mathcal{P}$ e sejam $a_{1}, \ldots, a_{m}$ as linhas de $A$ ativas em $\mathcal{F}$. Seja $c$ um vetor inteiro em $\operatorname{cone}\left(a_{1}, \ldots, a_{m}\right)$. Se considerarmos a equação de dualidade usual,

$$
\max \{c x: A x \leq b\}=\min \{y b: y \geq 0, y A=c\},
$$

podemos verificar que todo vetor $x$ de $\mathcal{F}$ atinge o máximo. De fato, a combinação não-negativa das linhas ativas que gera $c$ induz uma solução $y$ para o mínimo tal que todo vetor $x$ de $\mathcal{F}$ satisfaz $c x=y b$. 
Como estamos supondo que $A x \leq b$ é totalmente dual integral, sabemos que o mínimo admite uma solução ótima inteira, digamos $y$. Por folgas complementares, essa solução é nula em todas as coordenadas correspondentes a linhas não-ativas em $\mathcal{F}$. Logo, $c$ é combinação inteira não-negativa de $a_{1}, \ldots, a_{m}$. Portanto, tais vetores são um sistema gerador de Hilbert totalmente integral.

Suponha agora que $c$ é um vetor inteiro para o qual o mínimo existe. Seja $\mathcal{F}$ uma face minimal de $\{x: A x \leq b\}$ cujos vetores atingem $\max \{c x: A x \leq b\}$. Sejam $a_{1}, \ldots, a_{m}$ as linhas ativas em $\mathcal{F}$. Sabemos que $c$ pertence a cone $\left(a_{1}, \ldots, a_{m}\right)$, pois existe uma solução para o mínimo que é nula nas coordenadas que não são correspondentes a linhas ativas e tal solução é uma combinação não-negativa que gera $c$. Se os vetores $a_{1}, \ldots, a_{m}$ formam um sistema gerador de Hilbert totalmente integral, $c=\lambda_{1} a_{1}+\cdots+\lambda_{m} a_{m}$, onde $\lambda_{1}, \ldots, \lambda_{t}$ são inteiros não-negativos. Esses coeficientes induzem um vetor $y$, também inteiro e não-negativo, tal que $y A=c$ e $y b=y A x=c x$ para todo $x$ em $\mathcal{F}$.

Desse teorema segue uma caracterização para sistemas geradores de Hilbert totalmente integrais:

Corolário 5.6. Seja A uma matriz inteira. O conjunto das linhas de A é um sistema gerador de Hilbert totalmente integral se e somente se o sistema $A x \leq 0$ é totalmente dual integral.

Demonstração. Como $x:=0$ satisfaz $A x=0$, temos que $\{x: A x=0\}$ não é vazio e, portanto, é uma face do poliedro definido por $A x \leq 0$. Logo, pelo teorema 5.5, segue que as linhas de $A$ formam um sistema gerador de Hilbert totalmente integral quando $A x \leq 0$ é totalmente dual integral.

Suponha agora que as linhas de $A$ formam uma sistema gerador de Hilbert totalmente integral e seja $c$ um vetor tal que $\delta:=\max \{c x: A x \leq 0\}$ existe. Então existe $y \geq 0$ tal que $y A=c$ e $y 0=\delta$. Isso implica que $\delta=0$. Assim, como as linhas de $A$ formam um sistema gerador de Hilbert totalmente integral, existe um vetor inteiro não-negativo $y^{\prime}$ tal que $y^{\prime} A=c$ e $y^{\prime} 0=0=\delta$.

Podemos também adaptar naturalmente o algoritmo de Chandrasekaran a esse tipo de sistema:

Corolário 5.7. Existe um algoritmo polinomial que, dados uma matriz $A$ e vetores $b, c$ tais que o sistema $A x \leq b$ é totalmente dual integral, resolve o programa inteiro $\min \{y b: y A=c ; y \geq 0\}$.

Demonstração. Segue diretamente do teorema 4.10. Um algoritmo de programação linear é utilizado para identificar as linhas ativas e o algoritmo de Chandrasekaran é aplicado sobre essas linhas.

É importante observar que total dual integralidade é propriedade de sistemas, não de poliedros.

$$
\left[\begin{array}{rr}
1 & 1 \\
1 & 0 \\
1 & -1
\end{array}\right] x \leq\left(\begin{array}{l}
0 \\
0 \\
0
\end{array}\right) \quad \text { e } \quad\left[\begin{array}{rr}
1 & 1 \\
1 & -1
\end{array}\right] x \leq\left(\begin{array}{l}
0 \\
0
\end{array}\right)
$$

definem o mesmo poliedro, mas podemos verificar que o primeiro sistema é totalmente dual integral e o segundo não. De fato, um sistema totalmente dual integral usualmente é formado por mais restrições do que o necessário para definir o poliedro.

Originalmente, Edmonds E GiLes [1977] definiram poliedros totalmente dual integrais como sendo aqueles que podem ser definidos por um sistema totalmente dual integral. Esse conceito se tornou desnecessário quando Giles e Pulleyblank [1979] demonstraram o teorema a seguir:

Teorema 5.8. Para todo poliedro $\mathcal{P}$, existe um sistema totalmente dual integral $A x \leq b$ que define $\mathcal{P}$. Ademais, o vetor b desse sistema é inteiro se e somente se $\mathcal{P}$ é um poliedro inteiro. 
Demonstração. Para cada face minimal $\mathcal{F}$ de $\mathcal{P}$, defina $\mathcal{C}_{\mathcal{F}}$ como o cone gerado por todos os vetores $c$ tais que $\max \{c x: x \in \mathcal{P}\}$ é obtido por todo vetor em $\mathcal{F}$. Dada uma face minimal $\mathcal{F}$, sejam $a_{1}, \ldots, a_{m}$ o sistema gerador de Hilbert totalmente integral que gera $\mathcal{C}_{\mathcal{F}}$ e que sabemos existir.

Dado $x^{*} \in \mathcal{F}$, defina $\beta_{i}:=a_{i} x^{*}$ para todo $i$. Pela definição do cone, temos $\beta_{i}=\max \left\{a_{i} x: x \in \mathcal{P}\right\}$. Logo, $a_{1} x \leq \beta_{1}, \ldots, a_{m} x \leq \beta_{m}$ definem um sistema $\mathcal{S}_{\mathcal{F}}$ de desigualdades satisfeitas pelos vetores de $\mathcal{P}$. Seja $A x \leq b$ o sistema dado por $\bigcup_{\mathcal{F} \subseteq \mathcal{P}} \mathcal{S}_{\mathcal{F}}$. Temos que $\mathcal{P} \subseteq\{x: A x \leq b\}$. Por outro lado, temos também $\{x: A x \leq b\} \subseteq \mathcal{P}$. De fato, suponha que $x^{\prime} \notin \mathcal{P}$ para algum $x^{\prime}$ em $\{x: A x \leq b\}$. Então existem $a, \beta$ tais que $a x \leq \beta$ se $x \in \mathcal{P}$ e $a x^{\prime}>\beta$. Logo, temos

$$
\max \{a x: x \in \mathcal{P}\} \leq \beta<a x^{\prime}
$$

e, portanto, existe uma face minimal $\mathcal{F}$ cujos vetores são os que atingem $\max \{a x: x \in \mathcal{P}\}$. Mas isso é uma contradição, pois implica que $a$ está em $\mathcal{C}_{\mathcal{F}}$.

Essa construção resulta, portanto, em um sistema $A x \leq b$ tal que $\mathcal{P}=\{x: A x \leq b\}$. Pelo teorema 5.5, sabemos que esse sistema é totalmente dual integral. Por fim, podemos verificar a partir do teorema 5.4 que o vetor $b$ obtido é inteiro se e somente se $\mathcal{P}$ é um poliedro inteiro.

Dizemos que um sistema totalmente dual integral é minimal se todo subsistema próprio não é totalmente dual integral ou não determina o mesmo poliedro. SCHRIJVER [1981] estendeu o teorema anterior para o seguinte resultado, que é uma "versão totalmente dual integral" do corolário 2.18:

Corolário 5.9. Se um poliedro $\mathcal{P}$ tem dimensão plena, então existe um único sistema totalmente dual integral minimal $A x \leq b$ que determina $\mathcal{P}$. Ademais, $b$ é inteiro se e somente se $\mathcal{P}$ é inteiro.

Demonstração. Seja $\mathcal{F}$ uma face de $\mathcal{P}$ e seja $\mathcal{C}_{\mathcal{F}}$ definido como na demonstração do teorema anterior. Vamos demonstrar que $\mathcal{F}$ é um cone pontudo. Suponha por contradição que existe $c \in \mathcal{C}_{\mathcal{F}}$ tal que $-c \in \mathcal{C}_{\mathcal{F}}$ e $c$ é não-nulo. Pela construção de $\mathcal{C}_{\mathcal{F}}$, sabemos que existe $\delta$ tal que $\mathcal{P} \subseteq\{x: c x=\delta\}$, pois $\mathcal{F}$ contém nesse caso todos os vetores de $\mathcal{P}$ que maximizam $c x$ e $-c x$ ao mesmo tempo. Mas isso é uma contradição com a hipótese de que o poliedro $\mathcal{P}$ tem dimensão plena.

Podemos então concluir que todo cone $\mathcal{C}_{\mathcal{F}}$ definido como no teorema anterior é pontudo. Portanto, o resultado segue diretamente do teorema 4.9: utilizamos a base de Hilbert única na construção.

Na seção a seguir, consideraremos o problema de reconhecer um sistema totalmente dual integral.

\subsection{Algoritmos em dimensão fixa}

Como mencionamos anteriormente, resolver um programa inteiro é um problema NP-difícil. No caso de decidir se o poliedro definido por um dado sistema é inteiro, não se sabe nem se o problema pertence à classe NP. Mas esse problema é tratável se fixarmos o posto da matriz considerada:

Teorema 5.10. Para todo $r$ fixo, existe um algoritmo polinomial que, dado um sistema $A x \leq b$ tal que $\operatorname{posto}(A)=r$, verifica se o poliedro $\mathcal{P}:=\{x: A x \leq b\}$ é inteiro.

Demonstração. Seja $A^{\prime} x \leq b^{\prime}$ um subsistema de $A x \leq b$ composto por $r$ restrições. Através da eliminação gaussiana, podemos verificar se $A^{\prime} x=b^{\prime}$ admite solução e obter uma solução $x^{\prime}$ em caso positivo. Se $A^{\prime} x^{\prime} \leq b^{\prime}$, podemos concluir que $\left\{x: A^{\prime} x=b^{\prime}\right\}$ é uma face minimal de $\mathcal{P}$. Finalmente, podemos verificar se essa face contém um vetor inteiro através do algoritmo de Euclides. Logo, podemos decidir se $\mathcal{P}$ é um poliedro executando esse procedimento sobre não mais do que $\left(\begin{array}{l}n \\ r\end{array}\right)$ subsistemas de $A x \leq b$, onde $n$ é o número de linhas de $A$. Como $\left(\begin{array}{l}n \\ r\end{array}\right) \leq n^{r}$, o resultado segue. 
De fato, até mesmo o problema de resolver um programa inteiro é tratável quando o posto da matriz considerada é fixo. O teorema a seguir foi apresentado originalmente por LENSTRA [1983].

Teorema 5.11. Para todo $r$ fixo, existe um algoritmo polinomial que, dados um sistema Ax $\leq b$ tal que $\operatorname{posto}(A)=r$ e um vetor $c$, resolve o programa inteiro $\max \{c x: A x \leq b ; x$ inteiro $\}$.

Para encerrar este capítulo, vamos utilizar o algoritmo de Lenstra como uma "caixa-preta" para demonstrar que sistemas totalmente dual integrais também podem ser reconhecidos se fixarmos o posto da matriz. O teorema a seguir foi demonstrado por CoOK, LOVÁsz E SCHRIJVER [1984].

Teorema 5.12. Para todo $r$ fixo, existe um algoritmo polinomial que, dados vetores $a_{1}, \ldots, a_{m}$ tais que $\operatorname{dim}\left(a_{1}, \ldots, a_{m}\right)=r$, verifica se eles formam um sistema gerador de Hilbert totalmente integral.

Demonstração. Vamos primeiramente mostrar que podemos supor sem perda de generalidade que os vetores $a_{1}, \ldots, a_{m}$ geram todo o espaço e $\mathcal{C}:=\operatorname{cone}\left(a_{1}, \ldots, a_{m}\right)$ é pontudo. Seja $\mathcal{F}$ a única face minimal de $\mathcal{C}$ e seja $\mathcal{L}$ o fecho linear de $\mathcal{C}$. Sem perda de generalidade, suponha que $a_{1}, \ldots, a_{t}$ são os vetores dentre $a_{1}, \ldots, a_{m}$ que pertencem a $\mathcal{F}$. Esses vetores podem ser determinados em tempo polinomial, pois para todo $i$ temos que $a_{i}$ está em $\mathcal{F}$ se e somente se $-a_{i}$ está em $\mathcal{C}$. Podemos testar essa condição através de um programa linear simples.

Seja $n$ o inteiro tal que $a_{1}, \ldots, a_{m}$ pertencem a $\mathbb{R}^{n}$ e seja $d:=\operatorname{dim}(\mathcal{F})$. Por definição, temos que $r=\operatorname{dim}(\mathcal{L})$. Vamos provar que existe uma matriz unimodular $U \in \mathbb{Z}^{n \times n}$ tal que $\{y U: y \in \mathcal{F}\}$ é o conjunto de todos os vetores em $\mathbb{R}^{n}$ cujos últimos $n-d$ elementos são nulos e $\{y U: y \in \mathcal{L}\}$ é o conjunto de todos os em $\mathbb{R}^{n}$ cujos últimos $n-r$ elementos são nulos. Sejam $b_{1}, \ldots, b_{d}$ vetores linearmente independentes dentre $a_{1}, \ldots, a_{t}$ e sejam $b_{d+1}, \ldots, b_{r}$ vetores linearmente independentes dentre $a_{t+1}, \ldots, a_{m}$. Seja $B$ uma matriz cujas linhas são $b_{1}, \ldots, b_{r}$. A matriz unimodular $U$ tal que $B U$ está na forma normal de Hermite satisfaz as condições desejadas.

Seja $A$ a matriz cujas linhas são $a_{1}, \ldots, a_{m}$. Se $c$ é um vetor inteiro e $c=y A$, onde $y \geq 0$, então $c U$ também é um vetor inteiro e $c U=y A U$. Analogamente, se $c$ é um vetor inteiro e $c=y A U$, onde $y \geq 0$, então $c U^{-1}$ também é um vetor inteiro e $c U^{-1}=y A$. Logo, as linhas de $A$ formam um sistema gerador de Hilbert totalmente integral se e somente se as linhas de $A U$ formam um sistema gerador de Hilbert totalmente integral.

Podemos então supor sem perda de generalidade que $A=A U$, que $\mathcal{F}$ é o conjunto de todos os vetores em $\mathbb{R}^{n}$ cujos últimos $n-d$ elementos são nulos e que $\mathcal{L}$ é o conjunto de todos os vetores em $\mathbb{R}^{n}$ cujos últimos $n-r$ elementos são nulos. Como as últimas $n-r$ colunas de $A=A U$ são nulas, também podemos supor $n=r$. Sob essas hipóteses, podemos verificar que as linhas de $A$ formam um sistema gerador de Hilbert totalmente integral se e somente se as seguintes afirmações valem:

(i) os vetores $a_{1}, \ldots, a_{t}$ formam um sistema gerador de Hilbert totalmente integral cujo cone é $\mathcal{F}$;

(ii) se $A^{\prime}$ é a submatriz induzida pelas últimas $n-d$ colunas da matriz $A$, então as linhas de $A^{\prime}$ formam um sistema gerador de Hilbert totalmente integral.

Vamos demonstrar que a afirmação (i) é verdadeira se e somente se $\operatorname{ret}\left(a_{1}, \ldots, a_{t}\right)=\mathcal{F} \cap \mathbb{Z}^{n}$. A necessidade segue diretamente da definição de sistema gerador de Hilbert totalmente integral. Para verificar a suficiência, seja $c$ um vetor inteiro em $\mathcal{F}$. Se $\operatorname{ret}\left(a_{1}, \ldots, a_{t}\right)=\mathcal{F} \cap \mathbb{Z}^{n}$, então existem inteiros $\lambda_{1}, \ldots, \lambda_{t}$ tais que $c=\lambda_{1} a_{1}+\cdots+\lambda_{t} a_{t}$. Sabemos que, para todo $i=1, \ldots, t$, o vetor $-a_{i}$ pertence a $\mathcal{F}$. Portanto, tal $-a_{i}$ é gerado por uma combinação não-negativa de $a_{1}, \ldots, a_{t}$. 
A partir dos mesmos argumentos que utilizamos na descrição do algoritmo de Chandrasekaran, podemos verificar que existem $\mu_{1}, \ldots, \mu_{t}>0$ tais que $\mu_{1} a_{1}+\cdots+\mu_{t} a_{t}=0$. Logo, para um inteiro $\gamma$ adequado, temos que $\left(\lambda_{1}+\gamma \mu_{1}\right) a_{1}+\cdots+\left(\lambda_{t}+\gamma \mu_{t}\right) a_{t}$ é uma combinação inteira não-negativa que gera $c$ e a suficiência de $\operatorname{ret}\left(a_{1}, \ldots, a_{t}\right)=\mathcal{F} \cap \mathbb{Z}^{n}$ está demonstrada.

Para todo $i=1, \ldots, t$, seja $a_{i}^{\prime}$ o subvetor induzido pelos $d$ primeiros elementos de $a_{i}$. Temos então que $\mathcal{F} \cap \mathbb{Z}^{n}$ é o reticulado gerado pelos vetores $a_{1}, \ldots, a_{t}$ se e somente se a forma normal de Hermite da matriz cujas colunas são $a_{1}^{\prime}, \ldots, a_{t}^{\prime}$ é $\left[\begin{array}{ll}I & 0\end{array}\right]$. Logo, podemos verificar em tempo polinomial se $\operatorname{ret}\left(a_{1}, \ldots, a_{t}\right)=\mathcal{F} \cap \mathbb{Z}^{n}$. Portanto, o problema se reduz a verificar se a afirmação (ii) é verdadeira. Assim, o algoritmo pode supor que $a_{1}, \ldots, a_{m}$ geram todo o espaço e $\mathcal{C}$ é pontudo.

Vamos enfim descrever o algoritmo. Primeiramente, demonstraremos que $a_{1}, \ldots, a_{m}$ formam um sistema gerador de Hilbert totalmente integral se e somente se o único vetor inteiro que pertence a

$$
\mathcal{C}_{0}:=\left\{c \in \mathcal{C}: c-a_{i} \notin \mathcal{C} \text { para todo } i\right\}
$$

é o vetor nulo. Vamos provar a necessidade. Seja $c$ um vetor inteiro em $\mathcal{C}_{0}$. Se $a_{1}, \ldots, a_{m}$ formam um sistema gerador de Hilbert totalmente integral, então $c=\lambda_{1} a_{1}+\cdots+\lambda_{m} a_{m}$, onde $\lambda_{1}, \ldots, \lambda_{m}$ são inteiros não-negativos. Como $c-a_{i} \notin \mathcal{C}$, temos $\lambda_{1}=0, \ldots, \lambda_{m}=0$.

Vamos agora provar a suficiência. Seja $c$ um vetor inteiro em $\mathcal{C}$ e seja $\lambda_{1}$ o maior racional tal que $c-\lambda_{1} a_{1}$ pertence a $\mathcal{C}$. Como $\mathcal{C}$ é pontudo, $\lambda_{1}$ está bem definido. Defina agora $\lambda_{2}$ como o maior racional tal que $c-\left\lfloor\lambda_{1}\right\rfloor a_{1}-\lambda_{2} a_{2}$ pertence a $\mathcal{C}$ e assim sucessivamente até obtermos $\lambda_{1}, \ldots, \lambda_{m}$. Por construção, temos claramente que $c-\left\lfloor\lambda_{1}\right\rfloor a_{1}-\cdots-\left\lfloor\lambda_{m}\right\rfloor a_{m}$ é um vetor em $\mathcal{C}_{0}$. Logo, se $\mathcal{C}_{0} \cap \mathbb{Z}^{n}=\{0\}$, podemos concluir que $c=\left\lfloor\lambda_{1}\right\rfloor a_{1}+\cdots+\left\lfloor\lambda_{m}\right\rfloor a_{m}$, ou seja, $c \in \operatorname{int}$. cone $\left(a_{1}, \ldots, a_{m}\right)$.

Descreveremos um algoritmo que verifica se $\mathcal{C}_{0} \cap \mathbb{Z}^{n}=\{0\}$. Sejam $b_{1}, \ldots, b_{t} \neq 0$ vetores tais que

$$
\mathcal{C}=\left\{c: b_{j} c \geq 0 \text { para todo } j\right\} .
$$

Esses vetores formam a caracterização poliédrica de $\mathcal{C}$ e podem ser calculados como na demonstração do teorema 2.24. Como estamos supondo que $n$ é fixo, esse cálculo é polinomial. Temos então que

$$
\mathcal{C}_{0}=\left\{c \in \mathcal{C}: \text { para todo } i \text { existe } j \text { tal que } b_{j} c<b_{j} a_{i}\right\}
$$

Logo, se $\Phi$ denota o conjunto de todas as funções $\varphi:\{1, \ldots, m\} \rightarrow\{1, \ldots, t\}$, podemos verificar que

$$
\mathcal{C}_{0}=\bigcup_{\varphi \in \Phi}\left\{c: b_{j} c \geq 0 \text { para todo } j \text { e } b_{\varphi(i)} c<b_{\varphi(i)} a_{i} \text { para todo } i\right\} \text {. }
$$

O algoritmo desejado precisa verificar se nenhum dos $|\Phi|$ conjuntos dessa união contém um vetor inteiro não-nulo. A cardinalidade de $\Phi$ pode ser exponencialmente grande, mas demonstraremos a seguir que essa verificação pode ser feita em tempo polinomial.

Seja $\mathcal{X}$ o conjunto de todos os vetores que são solução de algum subsistema de $n$ equações lineares linearmente independentes dentre as que estão presentes no seguinte sistema de $t+m t$ equações:

$$
\begin{array}{ll}
b_{j} c=0 & \text { para todo } j \\
b_{j} c=b_{j} a_{i} & \text { para todo } i \text { e todo } j .
\end{array}
$$

Como estamos supondo que $n=r$ é um valor fixo, podemos obter esse conjunto $\mathcal{X}$ em tempo polinomial. Pelo mesmo argumento, também podemos obter em tempo polinomial uma coleção $\mathcal{S}$ de subconjuntos de $\mathcal{X}$ tal que $\left\{c_{1}, \ldots, c_{n}\right\} \subseteq \mathcal{X}$ pertence a $\mathcal{S}$ se e somente se valem as condições: 
(i) $c_{1}, \ldots, c_{n}$ pertencem a $\mathcal{C}$;

(ii) $c_{1}, \ldots, c_{n}$ são linearmente independentes;

(iii) para todo $i$ existe $j$ tal que $b_{j} c_{k} \leq b_{j} a_{i}$ para todo $k$.

Para cada subconjunto $\left\{c_{1}, \ldots, c_{n}\right\}$ de $\mathcal{X}$ em $\mathcal{S}$, vamos considerar:

$$
\sigma\left(c_{1}, \ldots, c_{n}\right):=\operatorname{conv}\left(0, c_{1}, \ldots, c_{n}\right) \backslash \operatorname{conv}\left(c_{1}, \ldots, c_{n}\right) .
$$

A partir da última reformulação de $\mathcal{C}_{0}$ apresentada, demonstraremos agora que:

$$
\mathcal{C}_{0}=\bigcup_{\left\{c_{1}, \ldots, c_{n}\right\} \in \mathcal{S}} \sigma\left(c_{1}, \ldots, c_{n}\right) .
$$

Primeiramente, sabemos que $\mathcal{C}_{0}$ é um conjunto limitado. De fato, $\mathcal{C}_{0} \subseteq \operatorname{par}\left(a_{1}, \ldots, a_{m}\right)$, pois caso contrário existe $c \in \mathcal{C}_{0}$ e algum $i$ tal que $c-a_{i} \in \mathcal{C}$. Seja $c^{\prime} \in \mathcal{C}_{0}$. Sabemos que $c^{\prime}$ pertence a

$$
\mathcal{C}_{\varphi}:=\left\{c: b_{j} c \geq 0 \text { para todo } j \text { e } b_{\varphi(i)} c<b_{\varphi(i)} a_{i} \text { para todo } i\right\}
$$

para alguma função $\varphi$ em $\Phi$. Portanto, temos que $\mathcal{C}_{\varphi}$ é limitado e não-vazio, assim como o poliedro

$$
\mathcal{C}_{\varphi}^{*}:=\left\{c: b_{j} c \geq 0 \text { para todo } j \text { e } b_{\varphi(i)} c \leq b_{\varphi(i)} a_{i} \text { para todo } i\right\} .
$$

Tome o maior $\lambda$ tal que $(1+\lambda) c^{\prime}$ pertence a $\mathcal{C}_{\varphi}^{*}$. Como $c^{\prime} \in \mathcal{C}_{\varphi}$, temos que $\lambda>0$. Ademais, temos também que $(1+\lambda) c^{\prime} \in \operatorname{conv}\left(x_{1}, \ldots, x_{l}\right)$, onde $x_{1}, \ldots, x_{l}$ são vetores em faces minimais de $\mathcal{C}_{\varphi}^{*}$ e $c^{\prime} \notin \operatorname{conv}\left(x_{1}, \ldots, x_{l}\right)$. Logo, pelo teorema de Carathéodory, temos $(1+\lambda) c^{\prime} \in \operatorname{cone}\left(c_{1}, \ldots, c_{n}\right)$, onde $c_{1}, \ldots, c_{n}$ são vetores linearmente independentes dentre $x_{1}, \ldots, x_{l}$. Portanto, segue diretamente que $c^{\prime} \in \operatorname{cone}\left(c_{1}, \ldots, c_{n}\right)$ e podemos concluir que $c^{\prime} \in \sigma\left(c_{1}, \ldots, c_{n}\right)$.

Suponha agora que $\left\{c_{1}, \ldots, c_{n}\right\}$ pertence a $\mathcal{S}$ e $c^{\prime}$ pertence a $\sigma\left(c_{1}, \ldots, c_{n}\right)$. Nesse caso, existe $\lambda>0$ tal que $(1+\lambda) c^{\prime} \in \operatorname{conv}\left\{c_{1}, \ldots, c_{n}\right\}$. Pela propriedade (i), temos que $b_{j} c^{\prime} \geq 0$ para todo $j$. Pela propriedade (iii), existe uma função $\varphi$ em $\Phi$ tal que $b_{\varphi(i)} c_{k} \leq b_{\varphi(i)} a_{i}$ para quaisquer $i, k$. Por fim, pela propriedade (ii) e pelo fato de que $b_{j} c_{k} \geq 0$ para quaisquer $j, k$, temos que $b_{\varphi(i)} a_{i}>0$ para todo $i$. De fato, se fosse verdade que $b_{\varphi(i)} a_{i}=0$ para algum $i$, teríamos $b_{\varphi}(i) c_{k}=0$ para todo $k$, o que seria uma contradição com a independência linear de $c_{1}, \ldots, c_{n}$. Portanto, como $(1+\lambda) b_{\varphi(i)} c^{\prime} \leq b_{\varphi(i)} a_{i}$ para todo $i$, temos que $b_{\varphi(i)} c^{\prime}<b_{\varphi(i)} a_{i}$ para todo $i$ e podemos concluir que $c^{\prime} \in \mathcal{C}_{\varphi}$.

Resta apenas descrever um algoritmo polinomial que, dado $\left\{c_{1}, \ldots, c_{n}\right\} \in \mathcal{S}$, verifica se existe algum vetor inteiro não-nulo em $\sigma\left(c_{1}, \ldots, c_{n}\right)$. Sabemos que $\mathcal{P}:=\operatorname{conv}\left(0, c_{1}, \ldots, c_{n}\right)$ é um poliedro e que $\mathcal{F}:=\operatorname{conv}\left(c_{1}, \ldots, c_{n}\right)$ é uma faceta desse poliedro. Podemos verificar que existe um vetor inteiro $c$ e um inteiro positivo $\delta$ tais que $\mathcal{F}=\{x: c x=\delta\}$. Logo, para todo $\alpha$ tal que $0<\alpha<1$, podemos verificar se $\sigma\left(c_{1}, \ldots, c_{n}\right)$ contém um vetor inteiro não-nulo resolvendo o programa inteiro

$$
\max \{c x: x \in \mathcal{P}, c x \leq \delta-\alpha ; x \text { inteiro }\} .
$$

Como estamos supondo que $r$ é um valor fixo, o algoritmo de Lenstra conclui a demonstração.

A partir desse teorema, podemos utilizar a estratégia de enumeração das faces minimais que foi descrita na demonstração do teorema 5.10 para verificar se um sistema é totalmente dual integral:

Corolário 5.13. Para todo $r$ fixo, existe um algoritmo polinomial que, dado um sistema Ax $\leq b$ tal que $\operatorname{posto}(A)=r$, decide se esse sistema é totalmente dual integral. 


\section{Capítulo 6}

\section{Versões inteiras do teorema de Carathéodory}

Neste capítulo, vamos considerar versões do teorema de Carathéodory para cones inteiros e bases de Hilbert. A primeira versão apresentada é um teorema de Cook, Fonlupt e Schrijver. Em seguida, apresentamos um refinamento de Sebő para esse teorema, suas conjecturas a respeito de versões mais fortes e demonstrações para casos particulares dessas conjecturas. Por fim, apresentamos um contra-exemplo para as conjecturas, obtido por Bruns, Gubeladze, Henk, Martin e Weismantel.

\subsection{Teorema de Cook, Fonlupt e Schrijver}

Vamos relembrar o teorema de Carathéodory, que nos estabelece um limitante superior para a quantidade de vetores necessários para se gerar vetores específicos em um dado cone.

Corolário 2.29. Seja $\mathcal{A}$ um conjunto de vetores. Se b pertence ao cone de $\mathcal{A}$, então b é gerado por uma combinação não-negativa de não mais do que $\operatorname{dim}(\mathcal{A})$ vetores em $\mathcal{A}$.

Corolário 2.30. Seja A uma matriz e sejam $b, c$ vetores. Se $\min \{y b: y A=c ; y \geq 0\}$ é um programa linear que admite solução, então existe uma solução y* para esse programa linear tal que não mais do que $\operatorname{posto}(A)$ elementos de $y^{*}$ são não-nulos.

Seria possível obtermos um resultado análogo para cones inteiros e bases de Hilbert? Para bases de Hilbert em geral, não apenas a resposta é negativa, como podemos construir um exemplo de base no qual o número de elementos necessários para se gerar um vetor no cone inteiro é arbitrariamente maior do que a dimensão. Considere $k$ primos positivos distintos $p_{1}, \ldots, p_{k}$ e $k$ inteiros $q_{1}, \ldots, q_{k}$ definidos da seguinte forma:

$$
q_{i}=\prod_{j \neq i} p_{j}=p_{1} \ldots p_{i-1} p_{i+1} \ldots p_{k} .
$$

Temos então que, para cada $i$, os divisores de $q_{i}$ são $p_{1}, \ldots, p_{i-1}, p_{i+1}, \ldots, p_{k}$. Logo, o único, e conseqüentemente o máximo, divisor comum de $q_{1}, \ldots, q_{k}$ é 1. Assim, pela identidade de Bézout,

$$
\mu_{1} q_{1}+\cdots+\mu_{k} q_{k}=1 \text {, onde } \mu_{1}, \ldots, \mu_{k} \text { são inteiros. }
$$

Vamos definir $\gamma_{1}, \ldots, \gamma_{k}$ como sendo os sinais de $\mu_{1}, \ldots, \mu_{k}$, ou seja, $\gamma_{i}=1$ se $\mu_{i} \geq 0$ e $\gamma_{i}=-1$ se $\mu_{i}<0$. Considere o conjunto $\mathcal{H}=\left\{\gamma_{1} q_{1}, \ldots, \gamma_{k} q_{k}\right\}$. 
Supondo $k \geq 2$, como temos que $q_{1}, \ldots, q_{k}$ são inteiros positivos, podemos verificar que existe $i$ tal que $\gamma_{i}>0$ e existe $j$ tal que $\gamma_{j}<0$. Logo, $\operatorname{cone}(\mathcal{H})=\mathbb{R}$ e $\operatorname{ret}(\mathcal{H})=\mathbb{Z}$. Ademais, as combinações

- $\left|\mu_{1}\right| \cdot\left(\gamma_{1} q_{1}\right)+\cdots+\left|\mu_{k}\right| \cdot\left(\gamma_{k} q_{k}\right)=1$;

- $1 \cdot \gamma_{j} q_{j}+\left(-\gamma_{j} q_{j}-1\right) \cdot 1=-1$,

nas quais $\left|\mu_{1}\right|, \ldots,\left|\mu_{k}\right|, 1$ e $\left(-\gamma_{j} q_{j}-1\right)$ são os coeficientes não-negativos, mostram que 1 e -1 pertencem a int. cone $(\mathcal{H})$, o que implica que int. cone $(\mathcal{H})=\mathbb{Z}$.

Podemos concluir então que int. $\operatorname{cone}(\mathcal{H})=\operatorname{cone}(\mathcal{H}) \cap \operatorname{ret}(\mathcal{H})=\mathbb{Z}$ e, portanto, que $\mathcal{H}$ é sistema gerador de Hilbert. Considere então combinações inteiras não-negativas que geram 1. Note que, se $\mathcal{H}^{\prime}$ é um subconjunto de $\mathcal{H}$ que não contém $\gamma_{i} q_{i}$, então $p_{i}$ é um divisor comum dos inteiros em $\mathcal{H}^{\prime}$. Logo, para qualquer subconjunto próprio de $\mathcal{H}$ o máximo divisor comum é maior do que 1.

Como a identidade de Bézout afirma que o máximo divisor comum é o menor inteiro que pode ser expresso como combinação inteira, podemos concluir que 1 não está no cone inteiro de nenhum subconjunto próprio de $\mathcal{H}$. Isso prova dois fatos: que $\mathcal{H}$ é uma base de Hilbert e que precisamos de $k$ vetores para gerar um vetor no cone inteiro, onde $k$ pode ser arbitrariamente grande.

Podemos obter resultados melhores se nos restringirmos a cones pontudos. Vamos supor que um conjunto $\mathcal{H}=\left\{h_{1}, \ldots, h_{m}\right\}$ é uma base de Hilbert tal que cone $(\mathcal{H})$ é pontudo. Seja $H$ a matriz cujas colunas são os vetores em $\mathcal{H}$. Sabemos nesse caso que, para todo $v \in \operatorname{cone}(\mathcal{H})$ e $c$ arbitrário, o programa linear $\max \{c x: H x=v ; x \geq 0\}$ admite solução. Em particular, temos o caso $c=1$, ou seja, $c x=\sum_{i=1}^{m} \lambda_{i}$, onde $x=\left(\lambda_{1}, \ldots, \lambda_{m}\right)$.

Portanto, existem coeficientes $\lambda_{1}, \ldots, \lambda_{m} \geq 0$ tais que $\lambda_{1} h_{1}+\cdots+\lambda_{m} h_{m}=v$ e sua soma é a maior possível. Ademais, pelo teorema de Carathéodory, também podemos supor que $\left\{h_{i}: \lambda_{i}>0\right\}$ é um conjunto linearmente independente. Dizemos que coeficientes satisfazendo essas propriedades são $v$-ótimos em relação a $\mathcal{H}$. Como a ordem dos vetores em $\mathcal{H}$ não importa, vamos supor sem perda de generalidade que $\left\{h_{i}: \lambda_{i}>0\right\}=\left\{h_{1}, \ldots, h_{k}\right\}$.

Baseando-se nesse conceito, Cook, Fonlupt E SCHRIJVER [1986] demonstraram o teorema a seguir. Ao longo desta seção, vamos denotar por $\{\alpha\}$ a parte fracionária de $\alpha$, ou seja, $\{\alpha\}:=\alpha-\lfloor\alpha\rfloor$.

Teorema 6.1. Seja $\mathcal{H}$ uma base de Hilbert e suponha que cone $(\mathcal{H})$ é pontudo. Se $v$ pertence ao cone inteiro de $\mathcal{H}$, então $v$ é gerado por uma combinação inteira não-negativa de não mais do que $2 \operatorname{dim}(\mathcal{H})-1$ vetores em $\mathcal{H}$.

Demonstração. Sejam $\lambda_{1}, \ldots, \lambda_{m}$ coeficientes $v$-ótimos. Como no teorema 4.6 , temos a decomposição

$$
v=\sum_{i=1}^{m}\left\lfloor\lambda_{i}\right\rfloor h_{i}+\sum_{i=1}^{m}\left\{\lambda_{i}\right\} h_{i}=\sum_{i=1}^{k}\left\lfloor\lambda_{i}\right\rfloor h_{i}+\sum_{i=1}^{k}\left\{\lambda_{i}\right\} h_{i}
$$

e sabemos que $\sum_{i=1}^{k}\left\{\lambda_{i}\right\} h_{i} \in \operatorname{cone}(\mathcal{H}) \cap \operatorname{ret}(\mathcal{H})$. Logo, como $\mathcal{H}$ é base de Hilbert, existem inteiros não-negativos $\alpha_{1}, \ldots, \alpha_{m}$ tais que $\sum_{i=1}^{m} \alpha_{i} h_{i}=\sum_{i=1}^{k}\left\{\lambda_{i}\right\} h_{i}$. Assim, podemos obter a igualdade

$$
v=\sum_{i=1}^{k}\left\lfloor\lambda_{i}\right\rfloor h_{i}+\sum_{i=1}^{m} \alpha_{i} h_{i}
$$

uma combinação inteira não-negativa dos vetores em $\mathcal{H}$ que gera $v$. Pela maximalidade de $\sum_{i=1}^{m} \lambda_{i}$, sabemos que $\sum_{i=1}^{m} \alpha_{i} \leq \sum_{i=1}^{k}\left\{\lambda_{i}\right\}$. Ademais, como $0 \leq\left\{\lambda_{i}\right\}<1$ para todo $i$, temos $\sum_{i=1}^{k}\left\{\lambda_{i}\right\}<k$. Portanto, como $\sum_{i=1}^{m} \alpha_{i}$ é inteiro, segue que o valor dessa soma é menor ou igual a $k-1$. Logo, existem no máximo $k+(k-1) \leq 2 \operatorname{dim}(\mathcal{H})-1$ coeficientes positivos em $(6.1)$. 
Segue diretamente uma conseqüência para total dual integralidade:

Corolário 6.2. Seja $A$ uma matriz e seja $b$ um vetor. Suponha que o sistema $A x \leq b$ é totalmente dual integral. Se c é um vetor inteiro e o programa linear $\min \{y b: y A=c ; y \geq 0\}$ admite solução, então existe uma solução inteira $y *$ para esse programa linear tal que não mais do que 2 posto $(A)-1$ elementos de $y^{*}$ são não-nulos.

Na demonstração acima, a maximização da soma dos coeficientes teve papel fundamental para obtermos os limitantes desejados, o que evidencia a necessidade do cone considerado ser pontudo. Vamos nos aprofundar um pouco mais nas restrições impostas pela maximização.

Tivemos na prova um vetor em $\operatorname{cone}\left(h_{1}, \ldots, h_{k}\right) \cap \operatorname{ret}(\mathcal{H}) \backslash\left\{h_{1}, \ldots, h_{k}\right\}$, no caso $\sum_{i=1}^{k}\left\{\lambda_{i}\right\} h_{i}$, que é gerado por uma combinação inteira não-negativa $\sum_{i=1}^{m} \alpha_{i} h_{i}$. Ao subtrairmos $\sum_{i=1}^{k}\left\{\lambda_{i}\right\} h_{i}$ da combinação original $\sum_{i=1}^{k} \lambda_{i} h_{i}$ e depois somarmos $\sum_{i=1}^{m} \alpha_{i} h_{i}$, garantimos que o vetor gerado pela combinação resultante ainda fosse $v$, devido à igualdade das duas combinações. Ademais, a nova soma de coeficientes após essas operações pode ser calculada por

$$
\sum_{i=1}^{k} \lambda_{i}+\left(\sum_{i=1}^{m} \alpha_{i}-\sum_{i=1}^{k}\left\{\lambda_{i}\right\}\right) .
$$

Isso mostra que a maximalidade de $\sum_{i=1}^{k} \lambda_{i}$ implica $\sum_{i=1}^{m} \alpha_{i} \leq \sum_{i=1}^{k}\left\{\lambda_{i}\right\}$. Ademais, como também sabemos que $\sum_{i=1}^{m} \alpha_{i} \geq 1$, podemos concluir a partir dessa restrição que $\sum_{i=1}^{k}\left\{\lambda_{i}\right\} \geq 1$.

Podemos utilizar esses argumentos porque a combinação resultante é não-negativa, ou seja, o vetor gerado pertence ao cone. Isso é garantido pelo fato de que $\lambda_{i} \geq\left\{\lambda_{i}\right\}$ para todo $i$, ou seja, os coeficientes subtraídos não são suficientemente grandes para que os coeficientes resultantes sejam negativos. Mas mesmo se não tivéssemos essa hipótese, ainda teríamos a nosso favor o fato de que $\lambda_{1}, \ldots, \lambda_{k}$ são estritamente positivos, o que nos permitiria "adaptar" o argumento com a soma de $\varepsilon\left(\sum_{i=1}^{m} \alpha_{i}-\sum_{i=1}^{k}\left\{\lambda_{i}\right\}\right)$ ao invés de $\left(\sum_{i=1}^{m} \alpha_{i}-\sum_{i=1}^{k}\left\{\lambda_{i}\right\}\right)$, onde $\varepsilon$ é suficientemente pequeno para garantir não-negatividade. Através dessa estratégia, que chamaremos de pivoteamento, podemos generalizar o limitante inferior para vetores arbitrários em cone $\left(h_{1}, \ldots, h_{k}\right) \cap \operatorname{ret}(\mathcal{H}) \backslash\left\{h_{1}, \ldots, h_{k}\right\}$.

Muitos dos lemas deste capítulo serão enunciados de uma forma um pouco menos auto-contida do que o usual, para evitarmos a repetição de definições longas que estaremos usando constantemente.

Lema 6.3. Seja $h=\sum_{i=1}^{k} \gamma_{i} h_{i} ; \gamma_{1}, \ldots, \gamma_{k} \geq 0$. Se $h \in \operatorname{cone}\left(h_{1}, \ldots, h_{k}\right) \cap \operatorname{ret}(\mathcal{H}) \backslash\left\{h_{1}, \ldots, h_{k}\right\}$, então $\sum_{i=1}^{k} \gamma_{i} \geq 1$.

Demonstração. Como $\mathcal{H}$ é base de Hilbert e $h \in \operatorname{cone}(\mathcal{H}) \cap \operatorname{ret}(\mathcal{H})$, sabemos que existem inteiros não-negativos $\alpha_{1}, \ldots, \alpha_{m}$ tais que $\sum_{i=1}^{m} \alpha_{i} h_{i}=h$. Segue que podemos gerar $v$ com

$$
\begin{aligned}
v & =\sum_{i=1}^{m} \lambda_{i} h_{i} \\
& =\sum_{i=1}^{m} \lambda_{i} h_{i}+(h-h) \\
& =\sum_{i=1}^{m} \lambda_{i} h_{i}+\left(\sum_{i=1}^{m} \alpha_{i} h_{i}-\sum_{i=1}^{k} \gamma_{i} h_{i}\right) \\
& =\sum_{i=1}^{m} \lambda_{i} h_{i}+\varepsilon\left(\sum_{i=1}^{m} \alpha_{i} h_{i}-\sum_{i=1}^{k} \gamma_{i} h_{i}\right) \text { para todo } \varepsilon>0 .
\end{aligned}
$$


Para $\varepsilon$ suficientemente pequeno, $\lambda_{i}+\varepsilon\left(\alpha_{i}-\gamma_{i}\right) \geq 0$ para todo $i \leq k$. Portanto, pela maximalidade de $\sum_{i=1}^{m} \lambda_{i}$, segue que $\sum_{i-1}^{k} \gamma_{i} \geq \sum_{i=1}^{m} \alpha_{i} \geq 1$.

Como poderíamos refinar esse limitante? Vamos considerar agora a possibilidade de obter a desigualdade estrita $\sum_{i=1}^{k} \gamma_{i}>1$. O primeiro passo natural nessa direção é verificar como podemos impedir o caso $\sum_{i=1}^{k} \gamma_{i}=1$. Nesse caso particular temos $\sum_{i=1}^{m} \alpha_{i}=1$, ou seja, $h=h_{j} \in \mathcal{H}$.

Para impedir esse caso, a maximalidade da soma parece ser insuficiente. No entanto, como ainda estamos considerando um cone pontudo, podemos experimentar estender a nossa estratégia de maximização para alguma outra função, que denotaremos por

$$
\sum_{i=1}^{m} c_{i} \lambda_{i}
$$

Por "estender" entenda-se maximizar essa nova função mas ainda sob a restrição de $v$-otimalidade. O efeito de um pivoteamento sob o valor dessa nova função pode ser descrito pela soma

$$
\sum_{i=1}^{m} c_{i} \lambda_{i}+\varepsilon\left(\sum_{i=1}^{m} c_{i} \alpha_{i}-\sum_{i=1}^{k} c_{i} \gamma_{i}\right)=\sum_{i=1}^{m} c_{i} \lambda_{i}+\varepsilon\left(c_{j}-\sum_{i=1}^{k} \gamma_{i} c_{i}\right) .
$$

Portanto, gostaríamos que $c_{j}>\gamma_{1} c_{1}+\cdots+\gamma_{k} c_{k}$ no caso em que $\sum_{i=1}^{k} \gamma_{i}=1$. Lembrando que $h_{j}$ é dado precisamente pela soma $\gamma_{1} h_{1}+\cdots+\gamma_{k} h_{k}$, podemos afirmar que o que queremos de $c_{1}, \ldots, c_{k}$ é que seus opostos satisfaçam uma desigualdade triangular estrita. Como estamos supondo que os vetores considerados são linearmente independentes, um candidato natural a essa nova função a ser maximizada é a norma do vetor. Vamos formalizar melhor essa idéia a seguir.

Lema 6.4. Para todo vetor $h=\sum_{i=1}^{k} \gamma_{i} h_{i} ; \gamma_{1}, \ldots, \gamma_{k} \geq 0$ em $\operatorname{cone}\left(h_{1}, \ldots, h_{k}\right) \cap \operatorname{ret}(\mathcal{H}) \backslash\left\{h_{1}, \ldots, h_{k}\right\}$, temos que $\sum_{i=1}^{k} \gamma_{i}>1$.

Demonstração. Sejam $\lambda_{1}, \ldots, \lambda_{m}$ coeficientes $v$-ótimos que maximizam $\sum_{i=1}^{m}-\left\|h_{i}\right\| \lambda_{i}$. Seja $h$ um vetor $\sum_{i=1}^{k} \gamma_{i} h_{i} \in \operatorname{cone}\left(h_{1}, \ldots, h_{k}\right) \cap \operatorname{ret}(\mathcal{H}) \backslash\left\{h_{1}, \ldots, h_{k}\right\}$, o que implica $\sum_{i=1}^{k} \gamma_{i} \geq 1$.

Suponha por contradição que $\sum_{i=1}^{k} \gamma_{i}=1$. Então existe $j>k$ tal que $h=h_{j}$. Portanto,

$$
w=\sum_{i=1}^{m} \lambda_{i} h_{i}=\sum_{i=1}^{m} \lambda_{i} h_{i}+\varepsilon\left(h_{j}-\sum_{i=1}^{k} \gamma_{i} h_{i}\right)
$$

Temos então novos coeficientes $v$-ótimos cujo valor da função é dado por

$$
\sum_{i=1}^{m}-\left\|h_{i}\right\| \lambda_{i}+\varepsilon\left(-\left\|h_{j}\right\| \cdot 1-\sum_{i=1}^{k}-\left\|h_{i}\right\| \gamma_{i}\right)=\sum_{i=1}^{m}-\left\|h_{i}\right\| \lambda_{i}+\varepsilon\left(-\left\|h_{j}\right\|+\sum_{i=1}^{k}\left\|h_{i}\right\| \gamma_{i}\right) .
$$

Por outro lado, como $h_{1}, \ldots, h_{k}$ são linearmente independentes, vale a desigualdade estrita

$$
\left\|h_{j}\right\|=\left\|\sum_{i=1}^{k} \gamma_{i} h_{i}\right\|<\sum_{i=1}^{k}\left\|\gamma_{i} h_{i}\right\|=\sum_{i=1}^{k}\left\|h_{i}\right\| \gamma_{i} .
$$

Mas isso é uma contradição com a maximalidade de $\sum_{i=1}^{m}-\left\|h_{i}\right\| \lambda_{i}$ e estamos feitos. 
Se nos restringirmos a vetores no paralelepípedo, podemos explorar uma propriedade de simetria para obter também um limitante superior, bastando tomar o "complementar" da combinação.

Corolário 6.5. Para todo vetor $h=\sum_{i=1}^{k} \gamma_{i} h_{i} ; 0 \leq \gamma_{1}, \ldots, \gamma_{k}<1 \mathrm{em} \operatorname{par}\left(h_{1}, \ldots, h_{k}\right) \cap \operatorname{ret}(\mathcal{H})$, temos que $1<\sum_{i=1}^{k} \gamma_{i}<k-1$.

Demonstração. Basta aplicar o teorema anterior sobre $\sum_{i=1}^{k}\left(1-\gamma_{i}\right) h_{i}$, que também está no cone.

Essa simetria foi utilizada por SЕвó [1990] para melhorar o teorema de Cook, Fonlupt e Schrijver:

Teorema 6.6. Seja $\mathcal{H}$ uma base de Hilbert e suponha que cone $(\mathcal{H})$ é pontudo. Se v pertence ao cone inteiro de $\mathcal{H}$, então $v$ é gerado por uma combinação inteira não-negativa de não mais do que $2 \operatorname{dim}(\mathcal{H})-2$ vetores em $\mathcal{H}$.

Demonstração. Vamos seguir a mesma linha da demonstração do teorema de Cook, Fonlupt e Schrijver. Sejam $\lambda_{1}, \ldots, \lambda_{m}$ coeficientes $v$-ótimos. Existem inteiros não-negativos $\alpha_{1}, \ldots, \alpha_{k} \operatorname{com}$

$$
v=\sum_{i=1}^{k}\left\lfloor\lambda_{i}\right\rfloor h_{i}+\sum_{i=1}^{k}\left\{\lambda_{i}\right\} h_{i}=\sum_{i=1}^{k}\left\lfloor\lambda_{i}\right\rfloor h_{i}+\sum_{i=1}^{m} \alpha_{i} h_{i} .
$$

Ainda seguindo a mesma linha, se provarmos que $\sum_{i=1}^{k}\left\{\lambda_{i}\right\}<k-1$ estamos feitos. Suponha por contradição que $\sum_{i=1}^{k}\left\{\lambda_{i}\right\} \geq k-1$. Podemos reformular essa desigualdade da seguinte forma:

$$
1 \geq k-\sum_{i=1}^{k}\left\{\lambda_{i}\right\}=\sum_{i=1}^{k} 1-\sum_{i=1}^{k}\left\{\lambda_{i}\right\}=\sum_{i=1}^{k}\left(1-\left\{\lambda_{i}\right\}\right) .
$$

Tome $\gamma_{i}:=1-\left\{\lambda_{i}\right\}$ para todo $i$. Temos então $0<\gamma_{i} \leq 1$. Conseqüentemente, o vetor $\sum_{i=1}^{k} \gamma_{i} h_{i}$ está em cone $\left(h_{1}, \ldots, h_{k}\right) \cap \operatorname{ret}(\mathcal{H}) \backslash\left\{h_{1}, \ldots, h_{k}\right\}$. Como não impomos nenhuma restrição sobre os coeficientes $v$-ótimos utilizados, podemos supor sem perda de generalidade que são como no corolário anterior. Mas isso é uma contradição, pois nesse caso tal vetor não devia existir.

Para encerrar esta seção, vamos considerar as implicações algorítmicas desses resultados. Como vimos, o cálculo de coeficientes $v$-ótimos nada mais é do que a resolução de um programa linear. Uma vez sabendo o valor máximo da soma dos coeficientes, resolver um segundo programa linear também nos permite obter coeficientes $v$-ótimos que maximizam $\sum_{i=1}^{m}-\left\|h_{i}\right\| \lambda_{i}$.

Resta então o problema de calcular $\alpha_{1}, \ldots, \alpha_{m}$. Mas podemos verificar que esse cálculo é simples observando-se uma propriedade forte derivada das demonstrações: limitar a soma dos coeficientes por $\sum_{i=1}^{k}\left\{\lambda_{i}\right\}$ serve para qualquer combinação inteira não-negativa dos vetores em $\mathcal{H}$ que gera esse vetor. Logo, podemos adotar uma estratégia "gulosa": basta subtrair vetores de $\mathcal{H}$ até atingir zero, desde que as subtrações preservem a pertinência no cone, o que pode ser verificado através de um programa linear. O número de iterações não é maior do que $\sum_{i=1}^{k}\left\{\lambda_{i}\right\}$.

Teorema 6.7. Seja $\mathcal{H}$ uma base de Hilbert e suponha que cone $(\mathcal{H})$ é pontudo. Se v pertence ao cone inteiro de $\mathcal{H}$, então $v$ é gerado por uma combinação inteira não-negativa de não mais do que $2 \operatorname{dim}(\mathcal{H})-2$ vetores em $\mathcal{H}$. Ademais, essa combinação pode ser obtida em tempo polinomial. 


\subsection{Conjecturas de Sebő}

Ao compararmos o teorema de Carathéodory com os resultados de Cook, Fonlupt e Schrijver e Sebő, cabe observar que o resultado não é completamente análogo, visto que os limitantes que foram obtidos são maiores do que a dimensão $\operatorname{se} \operatorname{dim}(\mathcal{H})>2$. Podemos nos perguntar a respeito de uma analogia mais completa:

Conjectura A. Seja $\mathcal{H}$ uma base de Hilbert e suponha que cone $(\mathcal{H})$ é pontudo. Se v pertence ao cone inteiro de $\mathcal{H}$, então $v$ é gerado por uma combinação inteira não-negativa de não mais do que $\operatorname{dim}(\mathcal{H})$ vetores em $\mathcal{H}$. Ademais, essa combinação pode ser obtida em tempo polinomial.

O teorema de Cook, Fonlupt e Schrijver prova a conjectura para $\operatorname{dim}(\mathcal{H})=1$ e a versão de Sebó prova a conjectura para $\operatorname{dim}(\mathcal{H})=2$.

Até o momento, estamos nos preocupando em encontrar um conjunto pequeno de vetores que contenham $v$ em seu cone inteiro, sem exigir nada a mais sobre esses vetores. Entretanto, como $\mathcal{H}$ é uma base de Hilbert, para a analogia ficar completa seria interessante que o subconjunto também fosse uma base de Hilbert. Além disso, o limitante do teorema de Carathéodory original é conseqüência de independência linear e qualquer conjunto linearmente independente é uma base de Hilbert. Podemos então formular uma conjectura mais ambiciosa:

Conjectura B. Seja $\mathcal{H}$ uma base de Hilbert tal que cone $(\mathcal{H})$ é pontudo e seja $v \in \operatorname{cone}(\mathcal{H}) \cap \operatorname{ret}(\mathcal{H})$. Então existe uma base de Hilbert linearmente independente $\mathcal{H}^{\prime} \subseteq \mathcal{H}$ tal que $v \in \operatorname{cone}\left(\mathcal{H}^{\prime}\right) \cap \operatorname{ret}\left(\mathcal{H}^{\prime}\right)$. Ademais, $\mathcal{H}^{\prime}$ pode ser obtida em tempo polinomial.

Como estamos nos referindo a sistemas geradores de Hilbert, poderíamos ter utilizado cones inteiros na formulação da conjectura, ao invés da intersecção entre o cone e o reticulado. A formulação usada, no entanto, enfatiza a importância do conjunto ser sistema gerador de Hilbert e também a possibilidade de buscarmos demonstrações mais simples, que não exigem obter uma combinação inteira não-negativa, apenas uma combinação inteira e uma combinação não-negativa.

Observemos que, se quisermos provar essa conjectura, não precisamos de uma demonstração cuja direção seja provar a existência de uma base de Hilbert suficientemente pequena. Podemos adotar uma estratégia mais simples, que é a de encontrar uma base estritamente menor contendo $v$ enquanto o conjunto for linearmente dependente. A conjectura então pode ser reformulada como

Conjectura C. Seja $\mathcal{H}$ um sistema gerador de Hilbert linearmente dependente tal que cone $(\mathcal{H})$ é pontudo e seja $v \in \operatorname{cone}(\mathcal{H}) \cap \operatorname{ret}(\mathcal{H})$. Então existe um sistema gerador de Hilbert $\mathcal{H}^{\prime} \subset \mathcal{H}$ tal que $v \in \operatorname{cone}\left(\mathcal{H}^{\prime}\right) \cap \operatorname{ret}\left(\mathcal{H}^{\prime}\right)$. Ademais, esse sistema pode ser obtido em tempo polinomial.

\section{Caso bidimensional}

Na tentativa de provar essas conjecturas, um ponto de partida é adotar a mesma estratégia vista na demonstração do teorema de Cook, Fonlupt e Schrijver, isto é, utilizar vetores com certas propriedades que podemos obter a partir do teorema de Carathéodory original.

Precisamente através desse teorema, sabemos que obter um subconjunto $\mathcal{H}^{\prime}$ de $\mathcal{H}$ cujo cone contenha o vetor $v$ é fácil. Mas como podemos modificar esse conjunto de modo a preservar essas propriedades enquanto o transformamos em um conjunto que é sistema gerador de Hilbert e contém $v$ em seu reticulado? Para transformar $\mathcal{H}^{\prime}$ um sistema gerador de Hilbert, uma primeira tentativa natural é utilizarmos a mesma idéia do teorema 4.6: unir $\operatorname{par}\left(\mathcal{H}^{\prime}\right) \cap \operatorname{ret}\left(\mathcal{H}^{\prime}\right)$ a $\mathcal{H}^{\prime}$. 
Entretanto, esse procedimento transforma $\mathcal{H}^{\prime}$ em um sistema gerador de Hilbert que gera $\operatorname{ret}\left(\mathcal{H}^{\prime}\right)$, e não $\operatorname{ret}(\mathcal{H})$, como queríamos. Adotemos então uma modificação natural dessa estratégia: ao invés de $\operatorname{par}\left(\mathcal{H}^{\prime}\right) \cap \operatorname{ret}\left(\mathcal{H}^{\prime}\right)$, vamos adicionar $\operatorname{par}\left(\mathcal{H}^{\prime}\right) \cap \operatorname{ret}(\mathcal{H})$. O resultado abaixo mostra que isso funciona, demonstrando que os paralelepípedos continuam tendo papel importante.

Teorema 6.8. Seja $\mathcal{H}$ um conjunto de vetores racionais, seja $v \in \operatorname{cone}(\mathcal{H}) \cap \operatorname{ret}(\mathcal{H})$ e seja $\mathcal{H}^{\prime} \subseteq \mathcal{H}$ tal que $v \in \operatorname{cone}\left(\mathcal{H}^{\prime}\right)$. Defina $\mathcal{H}^{\prime \prime}:=\mathcal{H}^{\prime} \cup\left(\operatorname{par}\left(\mathcal{H}^{\prime}\right) \cap \operatorname{ret}(\mathcal{H})\right)$. Então $\mathcal{H}^{\prime \prime}$ é um sistema gerador de Hilbert tal que $v \in \operatorname{cone}\left(\mathcal{H}^{\prime \prime}\right) \cap \operatorname{ret}\left(\mathcal{H}^{\prime \prime}\right)$.

Demonstração. Seja $\mathcal{H}=\left\{h_{1}, \ldots, h_{m}\right\}$ e sem perda de generalidade vamos supor $\mathcal{H}^{\prime}=\left\{h_{1}, \ldots, h_{k}\right\}$. Dados $\lambda_{1}, \ldots, \lambda_{k} \geq 0$ tais que $\lambda_{1} h_{1}+\cdots+\lambda_{k} h_{k}=v$, vamos considerar a decomposição

$$
v=\sum_{i=1}^{k}\left\lfloor\lambda_{i}\right\rfloor h_{i}+\sum_{i=1}^{k}\left\{\lambda_{i}\right\} h_{i} .
$$

Como $\sum_{i=1}^{k}\left\{\lambda_{i}\right\} h_{i} \in \operatorname{par}\left(\mathcal{H}^{\prime}\right) \cap \operatorname{ret}(\mathcal{H})$, segue que $v \in \operatorname{int}$. cone $\left(\mathcal{H}^{\prime \prime}\right)=\operatorname{cone}\left(\mathcal{H}^{\prime \prime}\right) \cap \operatorname{ret}\left(\mathcal{H}^{\prime \prime}\right)$.

Apesar do teorema acima nos fornecer uma receita para obtermos um sistema gerador de Hilbert que contém o dado vetor em seu cone inteiro, ele não é o suficiente para provarmos a conjectura $\mathrm{C}$ devido ao fato de que não estamos dando nenhuma garantia de que $\mathcal{H}^{\prime \prime}$ é um subconjunto próprio de $\mathcal{H}$. Na pior das hipóteses, ele nem sequer é um subconjunto. Num caso mais frustrante, ele é o próprio $\mathcal{H}$.

Quando não estamos nesses dois casos extremos, porém, o teorema está provado. Pensando justamente nessa direção, é interessante quando o conjunto $\operatorname{par}\left(\mathcal{H}^{\prime}\right) \cap \operatorname{ret}(\mathcal{H})$ é vazio. O corolário 6.5 estabelece restrições precisamente para elementos desse tipo de conjunto. Em um contexto em que essas restrições são suficientemente fortes, elas podem garantir que o conjunto é vazio, como no caso em que a dimensão do cone não é maior do que 2 .

Teorema 6.9. Se $\operatorname{dim}(\mathcal{H}) \leq 2$, as conjecturas $A, B$ e $C$ são verdadeiras.

Demonstração. Segue do corolário 6.5. Não pode existir $\sum_{i=1}^{k} \gamma_{i} h_{i} \in \operatorname{em} \operatorname{par}\left(\mathcal{H}^{\prime}\right) \cap \operatorname{ret}(\mathcal{H})$, pois caso contrário teríamos $1<\sum_{i=1}^{k} \gamma_{i}<k-1 \leq 1$. Segue que $\mathcal{H}^{\prime} \cup\left(\operatorname{par}\left(\mathcal{H}^{\prime}\right) \cap \operatorname{ret}(\mathcal{H})\right)$ é o próprio $\mathcal{H}^{\prime}$ e, portanto, a base de Hilbert linearmente independente que queríamos obter.

\section{Caso tridimensional}

Vamos então tentar estender o argumento para dimensão 3. Nesse caso podemos no máximo afirmar que $1<\sum_{i=1}^{k} \gamma_{i}<2$. Logo, a restrição não é mais suficiente para garantir que o conjunto é vazio. No entanto, existem inteiros não-negativos $\alpha_{1}, \ldots, \alpha_{m}$ tais que $\sum_{i=1}^{k} \gamma_{i} h_{i}=\sum_{i=1}^{m} \alpha_{i} h_{i}$ e $\sum_{i=1}^{m} \alpha_{i} \leq \sum_{i=1}^{k} \gamma_{i}$, como vimos na prova do teorema 6.1. Temos então $\sum_{i=1}^{m} \alpha_{i}=1$, o que implica $\sum_{i=1}^{k} \gamma_{i} h_{i} \in \mathcal{H}$. Podemos então concluir que $\mathcal{H}^{\prime} \cup\left(\operatorname{par}\left(\mathcal{H}^{\prime}\right) \cap \operatorname{ret}(\mathcal{H})\right) \subseteq \mathcal{H}$, o que ainda nos é favorável.

Porém, ainda não temos como concluir que o teorema vale para dimensão 3, pois como foi mencionado anteriormente existe a possibilidade de $\mathcal{H}^{\prime} \cup\left(\operatorname{par}\left(\mathcal{H}^{\prime}\right) \cap \operatorname{ret}(\mathcal{H})\right)$ ser o próprio $\mathcal{H}$. Vamos então considerar o caso em que $\mathcal{H}^{\prime}$ tem três vetores, $h_{1}, h_{2}, h_{3}$, existe $\sum_{i=1}^{3} \gamma_{i}=h \in \operatorname{par}\left(\mathcal{H}^{\prime}\right) \cap \operatorname{ret}(\mathcal{H})$ e $\mathcal{H}^{\prime} \cup\left(\operatorname{par}\left(\mathcal{H}^{\prime}\right) \cap \operatorname{ret}(\mathcal{H})\right)$. Em qualquer outro caso estamos feitos. 
Como o conjunto obtido não é um subconjunto próprio, vamos tentar então particioná-lo. A existência do vetor $h$ nos permite considerar a estratégia de utilizar esse vetor como um "ponto de particionamento" para o conjunto. Mais formalmente, consideremos os conjuntos

$$
\begin{aligned}
& \mathcal{H}_{1}=\left\{h, h_{2}, h_{3}\right\} \cup\left(\operatorname{par}\left(h, h_{2}, h_{3}\right) \cap \operatorname{ret}(\mathcal{H})\right) ; \\
& \mathcal{H}_{2}=\left\{h_{1}, h, h_{3}\right\} \cup\left(\operatorname{par}\left(h_{1}, h, h_{3}\right) \cap \operatorname{ret}(\mathcal{H})\right) ; \\
& \mathcal{H}_{3}=\left\{h_{1}, h_{2}, h\right\} \cup\left(\operatorname{par}\left(h_{1}, h_{2}, h\right) \cap \operatorname{ret}(\mathcal{H})\right) .
\end{aligned}
$$

Primeiramente, vamos provar que, se cada um desses conjuntos for um subconjunto de $\mathcal{H}$, então é um subconjunto próprio. Por simetria, precisamos apenas demonstrar isso para o primeiro.

Lema 6.10. $h_{1} \notin\left\{h, h_{2}, h_{3}\right\} \cup\left(\operatorname{par}\left(h, h_{2}, h_{3}\right) \cap \operatorname{ret}(\mathcal{H})\right)$.

Demonstração. Suponha por contradição que $h_{1}=\gamma^{\prime} h+\gamma_{2}^{\prime} h_{2}+\gamma_{3}^{\prime} h_{3}$ e $0 \leq \gamma^{\prime}, \gamma_{2}^{\prime}, \gamma_{3}^{\prime}<1$. Como os vetores $h_{1}, h_{2}, h_{3}$ são linearmente independentes, temos que $\gamma^{\prime}>0$. Logo,

$$
h=\frac{1}{\gamma^{\prime}} h_{1}-\frac{\gamma_{2}^{\prime}}{\gamma^{\prime}} h_{2}-\frac{\gamma_{3}^{\prime}}{\gamma^{\prime}} h_{3}
$$

Mas isso é uma contradição, pois $h=\sum_{i=1}^{3} \gamma_{i} h_{i}$ e essa combinação é única por independência.

Vamos agora provar que a união das três partes contém $\mathcal{H}$, como desejado.

Lema 6.11. $\mathcal{H} \subseteq \mathcal{H}_{1} \cup \mathcal{H}_{2} \cup \mathcal{H}_{3}$

Demonstração. Desconsiderando os elementos triviais $h_{1}, h_{2}, h_{3}, h$, é suficiente provar

$$
\begin{aligned}
\operatorname{par}\left(h_{1}, h_{2}, h_{3}\right) \cap \operatorname{ret}(\mathcal{H}) \backslash\{h\} \subseteq & \left(\operatorname{par}\left(h, h_{2}, h_{3}\right) \cap \operatorname{ret}(\mathcal{H})\right) \cup \\
& \left(\operatorname{par}\left(h_{1}, h, h_{3}\right) \cap \operatorname{ret}(\mathcal{H})\right) \cup \\
& \left(\operatorname{par}\left(h_{1}, h_{2}, h\right) \cap \operatorname{ret}(\mathcal{H})\right) .
\end{aligned}
$$

Considere então $h^{\prime}=\sum_{i=1}^{3} \gamma_{i}^{\prime} h_{i} \in \operatorname{par}\left(h_{1}, h_{2}, h_{3}\right) \cap \operatorname{ret}(\mathcal{H})$ tal que $h^{\prime} \neq h$. Primeiramente, vamos provar que $\gamma_{i}>\gamma_{i}^{\prime}$ para algum $i$. Suponha por contradição que $\gamma_{i} \leq \gamma_{i}^{\prime}$ para todo $i$. Temos nesse caso que $h^{\prime}-h=\sum_{i=1}^{3}\left(\gamma_{i}^{\prime}-\gamma_{i}\right) h_{i} \in \operatorname{par}\left(h_{1}, h_{2}, h_{3}\right) \cap \operatorname{ret}(\mathcal{H})$. Mas isso é uma contradição, pois implicaria também que $\sum_{i=1}^{k}\left(\gamma_{i}^{\prime}-\gamma_{i}\right)<2-1=1$.

Portanto, existe $i$ tal que $\gamma_{i}>\gamma_{i}^{\prime}$. Suponha então que $\gamma_{i}$ é o menor coeficiente que satisfaz essa propriedade e tome $\gamma:=\gamma_{i}^{\prime} / \gamma_{i}$. Segue que $0 \leq \gamma<1$. Sem perda de generalidade, vamos supor que $i=1$. Temos então que $h^{\prime}-\gamma h \in \operatorname{par}\left(h_{2}, h_{3}\right)$, o que conclui a demonstração.

Resta apenas demonstrar a inclusão inversa. Considerando o fato de que estamos trabalhando com conjuntos finitos, podemos adotar a estratégia de provar essa inclusão obtendo valores explícitos para a quantidade de elementos em cada conjunto e concluindo que a cardinalidade de um lado é a mesma do outro. Será útil aqui um resultado clássico de geometria dos números:

Teorema 6.12. Seja $A$ uma matriz quadrada não-singular inteira de ordem $n$ e sejam $a_{1}, \ldots, a_{n}$ as colunas de $A$. Então o número de elementos em $\operatorname{par}\left(a_{1}, \ldots, a_{n}\right) \cap \mathbb{Z}^{n}$ é $|\operatorname{det}(A)|$. 
Demonstração. Seja $A^{\prime}$ a transposta de $A$. Primeiramente, vamos transformar $A^{\prime}$ em sua forma normal de Hermite. Seja $U$ uma matriz unimodular tal que $A^{\prime} U$ é a forma normal de Hermite de $A$ e seja $U^{\prime}$ a transposta dessa matriz unimodular. A matriz transposta $U^{\prime} A$ é não-singular inteira triangular superior. Ademais, $A x$ é um vetor inteiro se e somente $U^{\prime} A x$ é um vetor inteiro, visto que $U^{\prime}$ e sua inversa são matrizes inteiras.

Logo, sendo $a_{1}^{\prime}, \ldots, a_{n}^{\prime}$ as colunas de $U^{\prime} A$, temos que a cardinalidade de $\operatorname{par}\left(a_{1}, \ldots, a_{n}\right) \cap \mathbb{Z}^{n}$ é igual à cardinalidade de $\operatorname{par}\left(a_{1}^{\prime}, \ldots, a_{n}^{\prime}\right) \cap \mathbb{Z}^{n}$. Vamos denotar por $\delta_{1}, \ldots, \delta_{n}$ os elementos da diagonal de $U^{\prime} A$ e utilizá-los para limitar o número de vetores $x=\left(\gamma_{1}, \ldots, \gamma_{n}\right)$ tais que $0 \leq \gamma_{i}<1$ para todo $i$ e $U^{\prime} A x$ é inteiro. Cabe lembrar que $U^{\prime} A$ é triangular superior.

Temos então que $\gamma_{n} \delta_{n}$ é um inteiro, ou seja, $\gamma_{n}=\mu / \delta_{n}$, onde $\mu$ é inteiro. Mas, como $0 \leq \gamma_{n}<1$, existem exatamente $\delta_{n}$ possibilidades para o valor de $\mu$ : $0, \ldots, \mu-1$. Tome então $\gamma_{i}$ com $i<n$ e seja $x:=\gamma_{i+1} \alpha_{i, i+1}+\cdots+\gamma_{n} \alpha_{i, n}$. Então $\gamma_{i} \delta_{i}+\{x\}$ é inteiro, ou seja, $\gamma_{i}=(\mu-\{x\}) / \delta_{i}$, onde $\mu$ é um inteiro. Como $0 \leq \gamma_{i}<1$, as possibilidades para $\mu$ nesse caso são $0, \ldots, \delta_{i}-1$ se $\{x\}=0$ e $1, \ldots, \delta_{i}$ se $\{x\}>0$. Em ambos os casos, o número de possibilidades é $\delta_{i}$.

Portanto, o número de elementos em $\operatorname{par}\left(a_{1}^{\prime}, \ldots, a_{n}^{\prime}\right) \cap \mathbb{Z}^{n}$ é $\delta_{1} \cdots \delta_{n}=\operatorname{det}\left(U^{\prime} A\right)=|\operatorname{det}(A)|$.

Para tirarmos proveito desse resultado, vamos supor sem perda de generalidade que $\mathcal{H} \subseteq \mathbb{Z}^{3}$ e que $\operatorname{ret}(\mathcal{H})=\mathbb{Z}^{3}$. De fato, podemos obter em tempo polinomial uma base de Hilbert isomorfa a $\mathcal{H}$ que satisfaz essas propriedades. Nesse novo contexto, podemos observar que

$$
\operatorname{par}\left(h_{1}, h_{2}, h_{3}\right) \cap \mathbb{Z}^{3} \backslash\{h\} \subseteq\left(\operatorname{par}\left(h, h_{2}, h_{3}\right) \cap \mathbb{Z}^{3}\right) \cup\left(\operatorname{par}\left(h_{1}, h, h_{3}\right) \cap \mathbb{Z}^{3}\right) \cup\left(\operatorname{par}\left(h_{1}, h_{2}, h\right) \cap \mathbb{Z}^{3}\right) .
$$

Em termos de cardinalidade, dessa inclusão segue diretamente a desigualdade

$$
\left|\operatorname{par}\left(h_{1}, h_{2}, h_{3}\right) \cap \mathbb{Z}^{3} \backslash\{h\}\right| \leq\left|\left(\operatorname{par}\left(h, h_{2}, h_{3}\right) \cap \mathbb{Z}^{3}\right) \cup\left(\operatorname{par}\left(h_{1}, h, h_{3}\right) \cap \mathbb{Z}^{3}\right) \cup\left(\operatorname{par}\left(h_{1}, h_{2}, h\right) \cap \mathbb{Z}^{3}\right)\right| .
$$

Note que, pelo teorema 6.12 e o fato do vetor nulo estar na intersecção dos três conjuntos escritos no lado direito da desigualdade, ela pode ser reescrita em termos de determinantes.

$$
\left|\operatorname{det}\left(h_{1}, h_{2}, h_{3}\right)\right|-1 \leq\left|\operatorname{det}\left(h, h_{2}, h_{3}\right)\right|+\left|\operatorname{det}\left(h_{1}, h, h_{3}\right)\right|+\left|\operatorname{det}\left(h_{1}, h_{2}, h\right)\right|-2 .
$$

Observe agora que $\operatorname{det}\left(h, h_{2}, h_{3}\right)=\operatorname{det}\left(\sum_{i=1}^{3} \gamma_{i} h_{i}, h_{2}, h_{3}\right)=\operatorname{det}\left(\gamma_{1} h_{1}, h_{2}, h_{3}\right)=\gamma_{1} \operatorname{det}\left(h_{1}, h_{2}, h_{3}\right)$. Aplicando o mesmo argumento sobre os outros conjuntos concluímos que a desigualdade equivale a

$$
\left|\operatorname{det}\left(h_{1}, h_{2}, h_{3}\right)\right|+1 \leq\left(\gamma_{1}+\gamma_{2}+\gamma_{3}\right) \cdot\left|\operatorname{det}\left(h_{1}, h_{2}, h_{3}\right)\right| .
$$

A demonstração estará concluída se provarmos que podemos escolher $h$ de forma a transformar a desigualdade acima em igualdade, pois assim a união dos conjuntos será $\mathcal{H}$. Se $h \in \operatorname{par}\left(h_{1}, h_{2}, h_{3}\right) \cap \mathbb{Z}$, então $H x=h$, onde $H$ é a matriz cujas colunas são $h_{1}, h_{2}, h_{3}$ e $x=\left(\gamma_{1}, \gamma_{2}, \gamma_{3}\right)$. Pela regra de Cramer, segue que cada $\gamma_{i}$ pode ser escrito como $\mu_{i} / \operatorname{det}\left(h_{1}, h_{2}, h_{3}\right)$, onde $\mu_{i}$ é um inteiro. Então

$$
\mu_{1}+\mu_{2}+\mu_{3}=\left(\gamma_{1}+\gamma_{2}+\gamma_{3}\right) \cdot \operatorname{det}\left(h_{1}, h_{2}, h_{3}\right) .
$$

Por outro lado, as restrições impostas sobre $h_{1}, h_{2}, h_{3}$ garantem que

$$
\left|\operatorname{det}\left(h_{1}, h_{2}, h_{3}\right)\right|<\left(\gamma_{1}+\gamma_{2}+\gamma_{3}\right) \cdot\left|\operatorname{det}\left(h_{1}, h_{2}, h_{3}\right)\right|<2 \cdot\left|\operatorname{det}\left(h_{1}, h_{2}, h_{3}\right)\right| .
$$

Segue que $\left|\mu_{1}+\mu_{2}+\mu_{3}\right|$ é algum elemento dentre $\left|\operatorname{det}\left(h_{1}, h_{2}, h_{3}\right)\right|+1, \ldots, 2\left|\operatorname{det}\left(h_{1}, h_{2}, h_{3}\right)\right|-1$, ou seja, há $\operatorname{det}\left(h_{1}, h_{2}, h_{3}\right)-1$ possibilidades para o valor de $\left|\sum_{i=1}^{3} \mu_{i}\right|$. Mas $\operatorname{det}\left(h_{1}, h_{2}, h_{3}\right)-1$ é precisamente o número de vetores não-nulos em $\operatorname{par}\left(h_{1}, h_{2}, h_{3}\right) \cap \mathbb{Z}$. Logo, se provarmos que

$$
s(h)=\left|\mu_{1}+\mu_{2}+\mu_{3}\right|
$$


é uma função injetora, provamos a existência de $h$ que satisfaz a igualdade desejada.

Suponha então que existem $h$ e $h^{\prime}$ tais que $s(h)=s\left(h^{\prime}\right)=s$ e vamos provar que $h=h^{\prime}$. Tome

$$
\begin{aligned}
h & =\gamma_{1} h_{1}+\gamma_{2} h_{2}+\gamma_{3} h_{3} \\
\bar{h} & =\left(1-\gamma_{1}\right) h_{1}+\left(1-\gamma_{2}\right) h_{2}+\left(1-\gamma_{3}\right) h_{3}=h_{1}+h_{2}+h_{3}-h \\
s(h) & =\left(\gamma_{1}+\gamma_{2}+\gamma_{3}\right)\left|\operatorname{det}\left(h_{1}, h_{2}, h_{3}\right)\right|=\left|\mu_{1}+\mu_{2}+\mu_{3}\right|=s \\
s(\bar{h}) & =\left(\left(1-\gamma_{1}\right)+\left(1-\gamma_{2}\right)+\left(1-\gamma_{3}\right)\right)\left|\operatorname{det}\left(h_{1}, h_{2}, h_{3}\right)\right|=3\left|\operatorname{det}\left(h_{1}, h_{2}, h_{3}\right)\right|-s \\
h^{\prime} & =\gamma_{1}^{\prime} h_{1}+\gamma_{2}^{\prime} h_{2}+\gamma_{3}^{\prime} h_{3} \\
\bar{h}^{\prime} & =\left(1-\gamma_{1}^{\prime}\right) h_{1}+\left(1-\gamma_{2}^{\prime}\right) h_{2}+\left(1-\gamma_{3}^{\prime}\right) h_{3}=h_{1}+h_{2}+h_{3}-h^{\prime} \\
s\left(h^{\prime}\right) & =\mu_{1}^{\prime}+\mu_{2}^{\prime}+\mu_{3}^{\prime}=s \\
s\left(\bar{h}^{\prime}\right) & =\left(\left(1-\gamma_{1}^{\prime}\right)+\left(1-\gamma_{2}^{\prime}\right)+\left(1-\gamma_{3}^{\prime}\right)\right)\left|\operatorname{det}\left(h_{1}, h_{2}, h_{3}\right)\right|=3\left|\operatorname{det}\left(h_{1}, h_{2}, h_{3}\right)\right|-s
\end{aligned}
$$

Temos que $h=h^{\prime}$ equivale a $h+\bar{h}^{\prime}=h_{1}+h_{2}+h_{3}$. Note que $s(h)+s\left(\bar{h}^{\prime}\right)=3\left|\operatorname{det}\left(h_{1}, h_{2}, h_{3}\right)\right|$. Logo, $h+\bar{h}^{\prime}=\lambda_{1} h_{1}+\lambda_{2} h_{2}+\lambda_{3} h_{3}$, onde $\lambda_{1}+\lambda_{2}+\lambda_{3}=3$. Cabe observar que $0<\lambda_{i}<2$ para todo $i$, pois $0<\left(1-\gamma_{i}^{\prime}\right) \leq 1$ e $0 \leq \gamma_{i}<1$ para todo $i$.

Resta provar que $\lambda_{1}, \lambda_{2}, \lambda_{3}$ são inteiros e, portanto, iguais a 1 . Suponha por contradição que $\sum_{i=1}^{3}\left\{\lambda_{i}\right\}>0$. Como $\sum_{i=1}^{3} \lambda_{i}=3$, então $\sum_{i=1}^{3}\left\{\lambda_{i}\right\}$ é inteiro. Mas isso é uma contradição, pois como $\sum_{i=1}^{3}\left\{\lambda_{i}\right\} h_{i} \in \operatorname{par}\left(h_{1}, h_{2}, h_{3}\right) \cap \operatorname{ret}(\mathcal{H})$, temos $1<\sum_{i=1}^{3}\left\{\lambda_{i}\right\}<2$.

Teorema 6.13. Se $\operatorname{dim}(\mathcal{H}) \leq 3$, todas as conjecturas são verdadeiras.

Isso conclui os estudos das conjecturas para dimensões específicas. Veremos a seguir que as conjecturas valem para certas classes de bases cuja caracterização não depende da dimensão.

\subsection{Classes especiais de bases de Hilbert}

Nesta seção, vamos explorar melhor algumas propriedades de bases de Hilbert que nos permitam provar que as conjecturas são válidas para certas classes especiais de bases. Não mais vamos limitar superiormente a dimensão como fizemos até agora, mas utilizaremos idéias já vistas e que foram fundamentais nas demonstrações para as conjecturas em dimensão limitada.

\section{Bases dependentes minimais}

Primeiramente, cabe lembrar que se o conjunto $\operatorname{par}\left(\mathcal{H}^{\prime}\right) \cap \operatorname{ret}(\mathcal{H})$ for vazio, a demonstração está praticamente concluída. No caso $\operatorname{dim}(\mathcal{H}) \leq 2$, chegamos à conclusão de que tal conjunto é vazio tirando proveito do fato de que um vetor pertencente a ele pode ser gerado por duas combinações lineares distintas. Vamos desenvolver melhor essa idéia. Um resultado nessa direção pode ser obtido lembrando de uma propriedade obtida na demonstração do caso $n=3$ : se $h \in \operatorname{par}\left(\mathcal{H}^{\prime}\right) \cap \operatorname{ret}(\mathcal{H})$ e $\bar{h}$ é o complementar de $h$, então $h+\bar{h} \in \operatorname{cone}\left(\mathcal{H}^{\prime}\right) \cap \operatorname{ret}(\mathcal{H})$ e $h+\bar{h}=h_{1}+\cdots+h_{k}$.

Lema 6.14. Seja $\mathcal{H}=\left\{h_{1}, \ldots, h_{m}\right\}$ uma base de Hilbert, seja $v \in \operatorname{cone}(\mathcal{H}) \cap \operatorname{ret}(\mathcal{H})$ e suponha que $\mathcal{H}^{\prime}=\left\{h_{1}, \ldots, h_{k}\right\}$ é um subconjunto linearmente independente de $\mathcal{H}$ tal que $v \in \operatorname{cone}\left(\mathcal{H}^{\prime}\right)$ mas $v \notin \operatorname{ret}\left(\mathcal{H}^{\prime}\right)$. Então existe um conjunto $I \subseteq\{1, \ldots, m\}$ tal que $\sum_{i \in I} h_{i}=\sum_{i \notin I} \alpha_{i} h_{i}$, onde $\alpha_{i}$ são inteiros não-negativos. 
Demonstração. Como $v \in \operatorname{cone}\left(\mathcal{H}^{\prime}\right)$ e $v \notin \operatorname{ret}\left(\mathcal{H}^{\prime}\right)$, existe um vetor não-nulo $h \in \operatorname{par}\left(\mathcal{H}^{\prime}\right) \cap \operatorname{ret}(\mathcal{H})$. Ademais, sabemos que $h_{1}+\cdots+h_{k}=h+\bar{h}$ é um vetor em $\operatorname{cone}\left(\mathcal{H}^{\prime}\right) \cap \operatorname{ret}(\mathcal{H})$. Logo, existem inteiros não-negativos $\alpha_{1}, \ldots, \alpha_{m}$ tais que $\sum_{i=1}^{k} h_{i}=\sum_{i=1}^{m} \alpha_{i} h_{i}$. Se para todo $i \leq k$ tal que $\alpha_{i}>0$ subtrairmos $h_{i}$ de ambos os lados da igualdade, obtemos o resultado desejado.

Outra propriedade similar pode ser obtida a partir de uma observação simples: como sempre podemos reescrever $\sum_{i=1}^{k} \gamma_{i} h_{i} \in \operatorname{par}(\mathcal{H}) \cap \operatorname{ret}(\mathcal{H})$ como uma combinação inteira não-negativa, basta multiplicarmos a igualdade por um inteiro adequado de forma a obter duas maneiras diferentes de gerar esse vetor através de uma combinação inteira não-negativa.

Lema 6.15. Seja $\mathcal{H}=\left\{h_{1}, \ldots, h_{m}\right\}$ uma base de Hilbert, seja $v \in \operatorname{cone}(\mathcal{H}) \cap \operatorname{ret}(\mathcal{H})$ e suponha que $\mathcal{H}^{\prime}=\left\{h_{1}, \ldots, h_{k}\right\}$ é um subconjunto linearmente independente de $\mathcal{H}$ tal que $v \in \operatorname{cone}\left(\mathcal{H}^{\prime}\right)$ mas $v \notin \operatorname{ret}\left(\mathcal{H}^{\prime}\right)$. Então existe um conjunto $I \subseteq\{1, \ldots, k\}$ tal que $\sum_{i \in I} \alpha_{i} h_{i}=\sum_{i \notin I} \alpha_{i} h_{i}$, onde $\alpha_{i}$ são inteiros não-negativos, $e \max \left\{\alpha_{i}: i \in I\right\}<\max \left\{\alpha_{i}: i \notin I\right\}$.

Demonstração. Como estamos supondo que $v \in \operatorname{cone}\left(\mathcal{H}^{\prime}\right)$ e $v \notin \operatorname{ret}\left(\mathcal{H}^{\prime}\right)$, então existe pelo menos um vetor não-nulo $h=\sum_{i=1}^{k} \gamma_{i} h_{i} \in \operatorname{par}\left(\mathcal{H}^{\prime}\right) \cap \operatorname{ret}(\mathcal{H})$ e existem inteiros não-negativos $\alpha_{1}, \ldots, \alpha_{m}$ tais que $h=\sum_{i=1}^{m} \alpha_{i} h_{i}$. Seja $q$ um inteiro tal que $\gamma_{i}=\mu_{i} / q$, onde $\mu_{i}$ é inteiro e $q>\mu_{i}$. Então temos que $\sum_{i=1}^{k} \mu_{i} h_{i}=\sum_{i=1}^{m} q \alpha_{i} h_{i}$. Para todo $i$, subtraindo $\min \left(\mu_{i}, q \alpha_{i}\right)$ de cada lado, estamos feitos.

Como então podemos aproveitar essa informação? Nos dois lemas, a igualdade obtida pode ser interpretada como uma combinação linear não-nula que gera o vetor nulo. Assim, podemos combinar os dois lemas nos casos em que há unicidade dessa combinação. Por exemplo:

Lema 6.16. Sejam $a_{1}, \ldots, a_{m+1}$ vetores linearmente dependentes e suponha que $a_{1}, \ldots, a_{m}$ são linearmente independentes. Então existe uma combinação linear não-nula desses vetores que gera o vetor nulo e essa combinação é única a menos de multiplicação por escalar.

Demonstração. Suponha por contradição que existem combinações não-nulas distintas, $\sum_{i=1}^{m+1} \lambda_{i} a_{i}$ e $\sum_{i=1}^{m+1} \lambda_{i}^{\prime} a_{i}$, que geram o vetor nulo. Como $a_{1}, \ldots, a_{m}$ são linearmente independentes, segue que $\lambda_{m+1}, \lambda_{m+1}^{\prime}>0$ e existe $\lambda$ tal que $\lambda_{m+1}-\lambda \lambda_{m+1}^{\prime}=0$. Por outro lado, como por hipótese as combinações são distintas, existe $i<m+1$ tal que $\lambda_{i}-\lambda \lambda_{i}^{\prime}=0$. Logo, $\sum_{i=1}^{m}\left(\lambda_{i}-\lambda \lambda_{i}\right) a_{i}$ é uma combinação não-nula que gera o vetor nulo, uma contradição com a independência.

Como então podemos aplicar os dois primeiros lemas sob a hipótese do terceiro? Consideremos a igualdade obtida a partir do primeiro: $\sum_{i \in I} h_{i}-\sum_{i \notin I} \alpha_{i}=0$. Se supormos que essa igualdade é equivalente à do segundo lema a menos de multiplicação por um escalar, podemos concluir que, devido à diferença dos máximos, existe $j$ tal que $\alpha_{j} \geq 2$.

Segue então que, para qualquer subconjunto que satisfaça as condições do primeiro lema, os índices aos quais ele se refere contém o conjunto $I$. Podemos então chegar à seguinte conclusão:

Teorema 6.17. As conjecturas $A, B$ e $C$ são verdadeiras se $\mathcal{H}=\left\{h_{1}, \ldots, h_{m+1}\right\}$ e $\operatorname{dim}(\mathcal{H})=m$.

Demonstração. Consideremos novamente a estratégia de obter coeficientes $v$-ótimos $\lambda_{1}, \ldots, \lambda_{m+1}$. Vamos utilizar a igualdade $\sum_{i \in I} h_{i}=\sum_{i \notin I} \alpha_{i} h_{i}$ do primeiro lema. Vamos aplicar o pivoteamento

$$
v=\sum_{i=1}^{m} \lambda_{i}^{\prime} h_{i}=\sum_{i=1}^{m} \lambda_{i} h_{i}+\left(\sum_{i \notin I} \lambda \alpha h_{i}-\sum_{i \in I} \lambda h_{i}\right), \text { onde } \lambda:=\max \left\{\lambda_{i}: i \in I\right\}
$$


Podemos verificar que, pela definição de $\lambda, \lambda_{i}^{\prime}=0$ para pelo menos um $i \in I$. Logo, temos que $\left\{i: \lambda_{i}^{\prime}>0\right\}$ não contém $I$. Ademais, $\left\{h_{i}: \lambda_{i}^{\prime}>0\right\}$ é linearmente independente, pois a existência de uma combinação não-nula que gera o vetor nulo violaria a unicidade, visto que o coeficiente correspondente a $h_{i}$ seria nulo. Portanto, os $\lambda_{i}^{\prime}$ induzem um subconjunto linearmente independente que contém $v$ no cone e não contém $I$. Como verificamos, isso implica que contém $v$ no reticulado.

\section{Bases fortes}

Para apresentar a segunda classe, vamos relembrar o algoritmo de Chandrasekaran. Vimos anteriormente que, para cones pontudos, podemos utilizar um algoritmo guloso para obter uma combinação inteira não-negativa que gera um vetor no cone inteiro de um sistema gerador de Hilbert. De maneira informal, o procedimento do algoritmo se baseia em subtrair vetores desse sistema, possivelmente multiplicados por um coeficiente, até chegarmos em um vetor na face minimal do cone. Para cones pontudos o algoritmo pode terminar nesse ponto, visto que o único vetor na face minimal é o vetor nulo.

O método de subtrair ao máximo possível um certo vetor da base também só pode ser aplicado devido ao fato do cone ser pontudo. Isso porque há um limite para o quanto ele pode ser subtraído. Observando o cone do ponto de vista poliédrico, a razão disso fica mais evidente. Sabemos que, para toda base de Hilbert $\mathcal{H}$, existe uma matriz $A$ tal que cone $(\mathcal{H})=\{x: A x \leq 0\}$. Ademais, se cone $(\mathcal{H})$ é pontudo, sabemos que $\{x: A x=0\}=\{0\}$. Disso segue que, se $h$ é um vetor não-nulo em cone $(\mathcal{H})$, então $a h<0$ para alguma linha $a$ de $A$. Portanto, se quisermos que $h^{\prime}-\lambda h$ esteja no cone, há um limite superior para $\lambda$, visto que $-a h$ é positivo.

Ademais, sabemos que, se $\mathcal{F}$ é uma face do cone, então existe uma partição das linhas de $A$ em duas submatrizes $A^{\prime}$ e $A^{\prime \prime}$ tais que $\mathcal{F}=\left\{x: A^{\prime} x=0 ; A^{\prime \prime} x \leq 0\right\}$. Logo, subtrair $\lambda h$ de $h^{\prime}$, onde $\lambda$ é o máximo possível, significa "puxar" o vetor original para uma face menor: mais uma restrição passa a ser satisfeita com igualdade. No algoritmo de Chandrasekaran, se os coeficientes obtidos são sempre inteiros, o fato de que o vetor chega na face minimal no final do algoritmo pode ser visto como o último de uma seqüência de passos que trazem o vetor para faces menores.

Mas essa garantia não existe para qualquer caso: o algoritmo contorna esse problema utilizando o chão do maior coeficiente ao invés do próprio. Bases de Hilbert que apresentam essa garantia, no entanto, são boas candidatas a satisfazerem as conjecturas: como a dimensão da face cai a cada passo, temos no final uma combinação não-negativa de vetores linearmente independentes.

Vamos formalizar melhor essa idéia: seja $\mathcal{F}$ uma face de cone $(\mathcal{H})$ e seja $h \in \mathcal{F} \cap \mathcal{H} \backslash\{0\}$. Dizemos que $h$ é um elemento forte de $\mathcal{F}$ se, para todo $h^{\prime} \in \mathcal{F} \cap \operatorname{ret}(\mathcal{H})$ para o qual $\mathcal{F}$ é a face minimal que contém $h^{\prime}$, vale que $h^{\prime}-h \in \operatorname{cone}(\mathcal{H})$. Elementos fortes tem a propriedade que desejamos:

Lema 6.18. Seja $h \in \operatorname{cone}(\mathcal{H}) \cap \operatorname{ret}(\mathcal{H})$ um elemento cuja face minimal é $\mathcal{F}$ e seja $h_{\mathcal{F}}$ um elemento forte de $\mathcal{F}$. Então o valor de $\max \left\{\lambda: h-\lambda h_{\mathcal{F}} \in \operatorname{cone}(\mathcal{H})\right\}$ é inteiro.

Demonstração. Suponha por contradição que um $\lambda$ atingindo esse máximo não é inteiro. Nesse caso, temos $\lfloor\lambda\rfloor<\lambda$, o que implica que subtrair $\lfloor\lambda\rfloor h_{\mathcal{F}}$ de $h$ não é o suficiente para satisfazer nenhuma nova restrição com igualdade. Mas então a face minimal de $h-\lfloor\lambda\rfloor h_{\mathcal{F}}$ também é $\mathcal{F}$, o que implica que $\left(h-\lfloor\lambda\rfloor h_{\mathcal{F}}\right)-h_{\mathcal{F}} \in \operatorname{cone}(\mathcal{H})$. Mas isso é uma contradição, pois $\lfloor\lambda\rfloor+1>\lambda$.

Dizemos que $\mathcal{H}$ é uma base de Hilbert forte se toda face de seu cone contém um elemento forte. Para bases fortes, portanto, basta subtrair elementos fortes até atingirmos a face minimal.

Teorema 6.19. As conjecturas $A, B$ e $C$ são verdadeiras para bases fortes. 
Demonstração. Vamos supor que $\mathcal{H}$ é uma base de Hilbert forte e demonstrar sob essa hipótese que a segunda conjectura vale. Sejam $\mathcal{F}_{1}, \ldots, \mathcal{F}_{k}$ faces de cone $(\mathcal{H})$ e $h_{1}, \ldots, h_{k} \in \mathcal{H}$ tais que $h_{i}$ é o elemento forte de $\mathcal{F}_{i}$. Suponha ainda que essas faces são definidas da seguinte forma:

- $v_{1}:=v$ e $v_{i}:=v_{i-1}-\lambda h_{i-1}$ se $i>1$, onde $\lambda:=\max \left\{\lambda: v_{i-1}-\lambda h_{i-1} \in \operatorname{cone}(\mathcal{H})\right\}$;

- $\mathcal{F}_{i}$ é a face minimal que contém $v_{i}$.

Como o lema anterior mostra, dessa definição segue um algoritmo polinomial para obtermos uma combinação inteira não-negativa de $h_{1}, \ldots, h_{k}$ que gera $v$. Ademais, qualquer combinação não-nula desses $k$ vetores está em $\mathcal{F}_{1}$, que não é a face minimal, ou seja, o vetor gerado pela combinação não pode ser o nulo. Portanto, podemos concluir que $h_{1}, \ldots, h_{k}$ são linearmente independentes.

Vamos provar que, se $w \in \operatorname{cone}(\mathcal{H}) \cap \operatorname{ret}(\mathcal{H})$ e $w \in \operatorname{cone}\left(h_{1}, \ldots, h_{k}\right)$, então $w \in \operatorname{ret}\left(h_{1}, \ldots, h_{k}\right)$. A prova é por indução em $k$. Se $k=1$, temos apenas $h_{1}$ e sabemos pelo lema que qualquer vetor em cone $\left(h_{1}\right) \cap \operatorname{ret}(\mathcal{H})$ é um múltiplo inteiro de $h_{1}$. Suponha então que $k>1$.

Se $h_{1}, \ldots, h_{k}$ não é uma base de Hilbert, existe $h=\sum_{i=1}^{k} \gamma_{i} h_{i} \in \operatorname{par}\left(h_{1}, \ldots, h_{k}\right) \cap \operatorname{ret}(\mathcal{H})$. Pela hipótese de indução, $h$ não pode estar em cone $\left(h_{2}, \ldots, h_{k}\right) \cap \operatorname{ret}(\mathcal{H})$, pois caso contrário deveria ser combinação inteira não-negativa de $h_{2}, \ldots, h_{k}$ e isso é uma contradição com o fato de $h_{1}, \ldots, h_{k}$ serem linearmente independentes: a combinação que gera $h$ deveria ser única.

Podemos então concluir que $\gamma_{1}>0$. Considere agora o vetor $h+h_{2}+\cdots+h_{k}$, que equivale a

$$
\gamma_{1} h_{1}+\left(\gamma_{2}+1\right) h_{2}+\cdots+\left(\gamma_{k}+1\right) h_{k} .
$$

A face minimal desse vetor é $\mathcal{F}_{1}$ e $\left(\gamma_{2}+1\right) h_{2}+\cdots+\left(\gamma_{k}+1\right) h_{k}$ pertence a $\mathcal{F}_{2}$. Logo, $\gamma_{1}$ é o maior $\lambda$ tal que $\left(h+h_{2}+\cdots+h_{k}\right)-\lambda h_{1}$ está em cone $(\mathcal{H})$. Mas isso é uma contradição, pois $h_{1}$ é o elemento forte de $\mathcal{F}_{1}$ e $\gamma_{1}$ por definição é fracionário.

Cabe observar que todos os passos descritos podem ser executados em tempo polinomial.

Um exemplo de base de Hilbert que pode facilmente ser reconhecida como uma base forte é descrita pelo lema a seguir:

Lema 6.20. Seja $\mathcal{H}$ uma base de Hilbert e suponha que, para todo $v \in$ int. cone $(\mathcal{H})$ e todo $h \in \mathcal{H}$, se existe $\varepsilon>0$ tal que $v-\varepsilon h \in \operatorname{cone}(\mathcal{H})$, então $v-h \in \operatorname{cone}(\mathcal{H})$. Então $\mathcal{H}$ é uma base forte e todos os vetores de $\mathcal{H}$ que estão em uma face de $\operatorname{cone}(\mathcal{H})$ são elementos fortes dessa face.

Demonstração. Seja $\mathcal{F}$ uma face minimal de $\operatorname{cone}(\mathcal{H})$ e seja $v$ um vetor em int. $\operatorname{cone}(\mathcal{H})$ tal que $\mathcal{F}$ é a face minimal do cone que o contém. Se $h \in \mathcal{H}$ é um vetor em $\mathcal{F}$, então podemos verificar que existe $\varepsilon>0$ tal que $v-\varepsilon h \in \operatorname{cone}(\mathcal{H})$. Pela hipótese, temos então $v-h \in \operatorname{int}$. $\operatorname{cone}(\mathcal{H})$.

Em termos de total dual integralidade, temos a partir desse lema o seguinte resultado:

Corolário 6.21. Seja $A$ uma matriz e sejam b, c vetores. Suponha que o sistema $A x \leq b$ é totalmente dual integral e que $\max \{c x: A x \leq 0\}$ admite solução. Se para toda restrição de $A x \leq b$ que é ativa na face correspondente às soluções desse programa linear existe uma solução y para o programa dual $\min \{y b: y A=c ; y \geq 0\}$ tal que o elemento de y correspondente a essa restrição é maior ou igual a 1 , então existe uma solução inteira para esse programa dual com não mais do que posto $(A)$ elementos não-nulos. 


\section{Bases livres de cruzamentos}

Para encerrar esta seção, vamos retomar uma propriedade que marcou presença constante nas demonstrações para os casos de dimensão 2 e 3 . Nessas demonstrações, pudemos ver que certas propriedades desejáveis podem ser obtidas se os vetores são escolhidos de forma a otimizar uma certa função: se tais propriedades não valessem, poderíamos trocar uma combinação linear por outra de forma a aumentar o valor da função. Seria possível estender essa estratégia a outros casos, bastando apenas escolher a função certa?

Os resultados obtidos no início desta seção sugerem uma direção que podemos considerar para essa estratégia: se certos vetores linearmente independentes $n \tilde{a} o$ tivessem as propriedades desejadas, a soma deles pode ser obtida através de uma outra combinação inteira não-negativa. Vamos dizer que uma base de Hilbert $\mathcal{H}$ é livre de cruzamentos se existe uma função $c: \mathcal{H} \rightarrow \mathbb{R}$ tal que, para todo subconjunto linearmente independente $\left\{h_{1}, \ldots, h_{k}\right\}$ de $\mathcal{H}$ cujo reticulado não é igual a $\operatorname{lin}\left(h_{1}, \ldots, h_{k}\right) \cap \operatorname{ret}(\mathcal{H})$, existem inteiros não-negativos $\alpha_{1}, \ldots, \alpha_{m}$ tais que

$$
\sum_{i=1}^{m} \alpha_{i} h_{i}=\sum_{i=1}^{k} h_{i} \quad \text { e } \quad \sum_{i=1}^{m} \alpha_{i} c\left(h_{i}\right)>\sum_{i=1}^{k} c\left(h_{i}\right) .
$$

Teorema 6.22. As conjecturas $A, B$ e $C$ são verdadeiras para bases livres de cruzamentos, desde que a função relacionada possa ser obtida em tempo polinomial.

Demonstração. Segue diretamente da estratégia de pivoteamento vista anteriormente.

Um exemplo de base livre de cruzamentos é justamente a primeira classe dessa seção.

Lema 6.23. Uma base de Hilbert $\mathcal{H}=\left\{h_{1}, \ldots, h_{m+1}\right\}$ tal que $\operatorname{dim}(\mathcal{H})=m$ é livre de cruzamentos.

Demonstração. Para bases desse tipo, vimos que existe $I \subseteq\{1, \ldots, m\}$ tal que

$$
\sum_{i \in I} h_{i}=\sum_{i \notin I} \alpha_{i} h_{i}
$$

onde $\alpha_{i}$ é um inteiro não-negativo. Ademais, para todo $J \subseteq\{1, \ldots, m\}$ tal que $\left\{h_{j}: j \in J\right\}$ é linearmente independente mas não gera o mesmo reticulado que $\mathcal{H}$, temos $I \subseteq J$.

Considere uma função $c$ qualquer que satisfaça $\sum_{i \notin I} \alpha_{i} c\left(h_{i}\right)>\sum_{i \in I} c\left(h_{i}\right)$. Então temos

$$
\begin{gathered}
\sum_{i \notin I} \alpha_{i} h_{i}+\sum_{i \in J \backslash I} h_{i}=\sum_{i \in I} h_{i}+\sum_{i \in J \backslash I} h_{i}=\sum_{i \in J} h_{i} \\
\sum_{i \notin I} \alpha_{i} c\left(h_{i}\right)+\sum_{i \in J \backslash I} c\left(h_{i}\right)>\sum_{i \in I} c\left(h_{i}\right)+\sum_{i \in J \backslash I} c\left(h_{i}\right)=\sum_{i \in J} c\left(h_{i}\right) .
\end{gathered}
$$

Para demonstrar o caso $\operatorname{dim}(\mathcal{H}) \leq 2$, também fizemos uso da otimização de uma função. Na verdade, fizemos uma otimização em duas fases: primeiro maximizando simplesmente a soma dos coeficientes, ou seja o custo associado a cada vetor nesse caso foi simplesmente 1, e sob a restrição de estar maximizando essa soma buscamos minimizar a soma das normas.

Para podermos concluir que bases de Hilbert cuja dimensão não é maior do que 2 são livres de cruzamentos, devemos conseguir essa otimização em duas fases através de uma única função. Vamos 
então associar ao vetor $h_{i}$ o custo $\left(1-\varepsilon\left\|h_{i}\right\|\right)$, onde $\varepsilon$ é um valor positivo pequeno. A idéia é que maximizar $\sum_{i=1}^{m} \lambda_{i}\left(1-\varepsilon\left\|h_{i}\right\|\right)$ ao mesmo tempo maximiza $\sum_{i=1}^{m} \lambda_{i}$ e minimiza $\sum_{i=1}^{m} \lambda_{i}\left\|h_{i}\right\|$, sendo que um $\varepsilon$ suficientemente pequeno prioriza a primeira maximização.

Lema 6.24. Bases de Hilbert de dimensão menor ou igual a 2 são livres de cruzamentos.

Demonstração. Se um subconjunto linearmente independente $\left\{h_{1}, \ldots, h_{k}\right\}$ não preserva o reticulado de $\mathcal{H}$, então existe $h=\sum_{i=1}^{k} \gamma_{i} h_{i} \in \operatorname{par}\left(h_{1}, \ldots, h_{k}\right) \cap \operatorname{ret}(\mathcal{H})$. Se $\bar{h}=\sum_{i=1}^{k}\left(1-\gamma_{i}\right) h_{i}$, temos

$$
h+\bar{h}=h_{1}+\cdots+h_{k}
$$

e sabemos que $h, \bar{h} \in \operatorname{cone}(\mathcal{H}) \cap \operatorname{ret}(\mathcal{H}) . \quad$ Logo, $h=\sum_{i=1}^{m} \alpha_{i} h_{i}$ e $\bar{h}=\sum_{i=1}^{m} \alpha_{i}^{\prime} h_{i}$, onde $\alpha_{i}, \alpha_{i}^{\prime}$ são inteiros não-negativos. Definindo $c\left(h_{i}\right)$ como sendo $\left(1-\varepsilon\left\|h_{i}\right\|\right)$, temos

$$
\begin{gathered}
\sum_{i=1}^{k} c\left(h_{i}\right)=\sum_{i=1}^{k}\left(1-\varepsilon\left\|h_{i}\right\|\right)=k-\varepsilon \sum_{i=1}^{k}\left\|h_{i}\right\| \\
\sum_{i=1}^{m} \alpha_{i} c\left(h_{i}\right)+\sum_{i=1}^{m} \alpha_{i}^{\prime} c\left(h_{i}\right)=\left(\sum_{i=1}^{m} \alpha_{i}+\sum_{i=1}^{m} \alpha_{i}^{\prime}\right)-\varepsilon\left(\sum_{i=1}^{m} \alpha_{i}\left\|h_{i}\right\|+\sum_{i=1}^{m} \alpha_{i}^{\prime}\left\|h_{i}\right\|\right) .
\end{gathered}
$$

Por integralidade, $\left(\sum_{i=1}^{m} \alpha_{i}+\sum_{i=1}^{m} \alpha_{i}^{\prime}\right) \geq 2 \geq k$. Se $\left(\sum_{i=1}^{m} \alpha_{i}+\sum_{i=1}^{m} \alpha_{i}^{\prime}\right)>k$, então, supondo que $\varepsilon$ seja suficientemente pequeno para relevar a subtração de $\varepsilon\left(\sum_{i=1}^{m} \alpha_{i}\left\|h_{i}\right\|+\sum_{i=1}^{m} \alpha_{i}^{\prime}\left\|h_{i}\right\|\right)$, o custo no lado esquerdo é maior. Vamos então considerar o caso $\left(\sum_{i=1}^{m} \alpha_{i}+\sum_{i=1}^{m} \alpha_{i}^{\prime}\right)=k=2$ :

$$
h+\bar{h}=h_{1}+h_{2} \text {, onde } h, \bar{h} \in \mathcal{H} .
$$

Queremos provar que $\|h\|+\|\bar{h}\|<\left\|h_{1}\right\|+\left\|h_{2}\right\|$. Mas isso segue da desigualdade triangular:

$$
\begin{aligned}
\|h\|+\|\bar{h}\| & =\left\|\gamma_{1} h_{1}+\gamma_{2} h_{2}\right\|+\left\|\left(1-\gamma_{1}\right) h_{1}+\left(1-\gamma_{2}\right) h_{2}\right\| \\
& <\left\|\gamma_{1} h_{1}\right\|+\left\|\gamma_{2} h_{2}\right\|+\left\|\left(1-\gamma_{1}\right) h_{1}\right\|+\left\|\left(1-\gamma_{2}\right) h_{2}\right\| \\
& =\left\|h_{1}\right\|+\left\|h_{2}\right\| .
\end{aligned}
$$

A desigualdade estrita segue do fato de que $\left(1-\gamma_{1}\right) h_{1}$ e $\left(1-\gamma_{2}\right) h_{2}$ são linearmente independentes.

Por fim, consideremos o caso de bases fortes. Segue diretamente da definição que se uma base de Hilbert é forte então todos os seus elementos são fortes. De fato, suponha que $h$ é um elemento de uma base forte que não é forte. Seja $\mathcal{F}$ a face minimal de $h$ e $h_{\mathcal{F}}$ o elemento forte de $\mathcal{F}$. Segue que $h-h_{\mathcal{F}}$ é um vetor não-nulo no cone e reticulado da base, uma contradição.

Considere então que $h_{\mathcal{F}}$ é o elemento forte da face minimal $\mathcal{F}$ que contém a soma $h_{1}+\cdots+h_{k}$. Todos esses vetores são elementos fortes em $\mathcal{F}$, mas apenas $h_{\mathcal{F}}$ é o elemento forte de $\mathcal{F}$. Mais formalmente, todos os vetores que não são $h_{\mathcal{F}}$ se referem a faces estritamente menores. Podemos então considerar uma função que é proporcional à dimensão da face ao qual um elemento forte se refere, o que garante aumento do custo quando reescrevemos a soma em termos de elementos fortes.

Lema 6.25. Bases de Hilbert fortes são livres de cruzamentos. 
Demonstração. Seja $\mathcal{H}$ uma base de Hilbert forte e $h_{1}, \ldots, h_{k}$ vetores linearmente independentes de $\mathcal{H}$ que não preservam o reticulado. Existe $h=\sum_{i=1}^{k} \gamma_{i} h_{i} \in \operatorname{par}\left(h_{1}, \ldots, h_{k}\right) \cap \operatorname{ret}(\mathcal{H}) \backslash\{0\}$.

Seja $\mathcal{F}$ a face minimal de $h_{1}+\cdots+h_{k}$ e seja $h_{\mathcal{F}}$ o elemento forte de $F$. Primeiramente, vamos supor que $h_{1}, \ldots, h_{k} \neq h_{F}$. Nesse caso, as faces minimais referentes a $h_{1}, \ldots, h_{k}$ são estritamente menores do que $\mathcal{F}$, ou seja, a dimensão dessas faces é no máximo $\operatorname{dim}(\mathcal{F})-1$. Considere então que cada $h_{i}$ está associado a um custo $c\left(h_{i}\right)=(\operatorname{dim}(\mathcal{H})+1)^{\operatorname{dim}\left(\mathcal{F}_{i}\right)}$. Nesse caso,

$$
c\left(h_{1}\right)+\cdots+c\left(h_{k}\right) \leq k(\operatorname{dim}(\mathcal{H})+1)^{\operatorname{dim}(\mathcal{F})-1} \leq \operatorname{dim}(\mathcal{H})(\operatorname{dim}(\mathcal{H})+1)^{\operatorname{dim}(\mathcal{F})-1} .
$$

Mas temos que $c\left(h_{\mathcal{F}}\right)=(\operatorname{dim}(\mathcal{H})+1)^{\operatorname{dim}(\mathcal{F})}$, portanto estamos feitos nesse caso, visto que o custo de $h_{\mathcal{F}}$ sozinho é superior ao da soma $h_{1}+\cdots+h_{k}$ e sabemos que essa soma pode ser reescrita como uma combinação inteira não-negativa de elementos fortes, dentre eles $h_{\mathcal{F}}$.

Vamos supor agora, sem perda de generalidade, que $h_{1}=h_{\mathcal{F}}$ e $h_{2}, \ldots, h_{k} \neq h_{\mathcal{F}}$. Sabemos que o maior $\lambda$ tal que $\left(h_{1}+\cdots+h_{k}\right)-\lambda h_{1} \in \operatorname{cone}(\mathcal{H})$ é inteiro e não-negativo. Suponha que $\lambda=1$. Nesse caso, a dimensão da face minimal de $h_{2}+\cdots+h_{k}$ é estritamente menor do que a da soma original. Através de um argumento indutivo análogo ao da demonstração original para as conjecturas no caso de bases fortes, podemos concluir que isso é uma contradição com a existência de $h$.

Resta considerar o caso em que $\lambda \geq 2$. Note que o custo da soma nesse caso é dado por

$$
c\left(h_{1}\right)+\cdots+c\left(h_{k}\right) \leq(\operatorname{dim}(\mathcal{H})+1)^{\operatorname{dim}(\mathcal{F})}+(k-1)(\operatorname{dim}(\mathcal{H})+1)^{\operatorname{dim}(\mathcal{F})-1}<2(\operatorname{dim}(\mathcal{H})+1)^{\operatorname{dim}(\mathcal{F})}
$$

e $2(\operatorname{dim}(\mathcal{H})+1)^{\operatorname{dim}(\mathcal{F})}$ limita inferiormente o custo de $\alpha_{1} h_{1}$ no lado direito.

Cabe observar então que para todos os casos em que provamos a validade da segunda conjectura, também pudemos provar que a base é livre de cruzamentos. Podemos então nos perguntar se

Conjectura D. Toda base de Hilbert é livre de cruzamentos e a função de descruzamento relacionada pode ser calculada em tempo polinomial.

Se essa conjectura fosse verdade, todas as outras também seriam. Todas as quatro conjecturas e as demonstrações para casos particulares presentes neste capítulo foram apresentadas por SEBÖ [1990].

\subsection{Contra-exemplo}

Vamos relembrar as relações entre as conjecturas: a conjectura $\mathrm{B}$ é equivalente à conjectura $\mathrm{C}$ e estas implicam a conjectura A. Por outro lado, a conjectura D implica a conjectura B e a conjectura C. Provando a conjectura D, provamos todas. Desprovando a conjectura A, desprovamos todas.

Foi precisamente através de um contra-exemplo para a conjectura A que todas as conjecturas foram desprovadas por Bruns, Gubeladze, Henk, Martin e Weismantel [1999], estendendo um resultado algébrico de Bruns E Gubeladze [1999]. Vamos encerrar este capítulo com esse contra-exemplo, que possui uma ressalva: algumas de suas propriedades foram demonstradas através de força bruta, resolvendo-se alguns subproblemas com o auxílio de um computador.

O contra-exemplo consiste no seguinte conjunto de vetores inteiros em $\mathbb{R}^{6}$ :

$$
\begin{aligned}
& z_{1}=(0,1,0,0,0,0), \quad z_{6}=(1,0,2,1,1,2), \\
& z_{2}=(0,0,1,0,0,0), \quad z_{7}=(1,2,0,2,1,1), \\
& z_{3}=(0,0,0,1,0,0), \quad z_{8}=(1,1,2,0,2,1), \\
& z_{4}=(0,0,0,0,1,0), \quad z_{9}=(1,1,1,2,0,2), \\
& z_{5}=(0,0,0,0,0,1), \quad z_{10}=(1,2,1,1,2,0) .
\end{aligned}
$$


Como $z_{6}-2 z_{2}-z_{3}-z_{4}-2 z_{5}=(1,0,0,0,0,0)$, segue que o reticulado gerado por esses vetores é $\mathbb{Z}^{6}$ e que o cone tem dimensão 6. Como todos os vetores são não-negativos, sabemos também que o cone gerado é pontudo. Por fim, a presença de 1 em todos os vetores e a posição dos zeros evidencia que não podem ser escrito como soma de vetores na intersecção do cone com o reticulado.

Sabemos então que todos os dez vetores estão em uma base de Hilbert que gera o mesmo cone e reticulado. Vimos anteriormente que tal base está contida em $\operatorname{par}(\mathcal{H}) \cap \operatorname{ret}(\mathcal{H})=\operatorname{par}(\mathcal{H}) \cap \mathbb{Z}^{6}$. Se para todo vetor $w$ nesse conjunto existir $z_{i}$ tal que $w-z_{i} \in \operatorname{cone}(\mathcal{H})$, poderíamos concluir que qualquer vetor no conjunto que não for um dos dez geradores é soma de dois outros e, portanto, não está na base de Hilbert. Disso seguiria que os dez vetores são precisamente a base de Hilbert. Essa propriedade $e ́$ verdadeira e foi provada por "força bruta".: todos os vetores no conjunto $\operatorname{par}(\mathcal{H}) \cap \operatorname{ret}(\mathcal{H})$ foram calculados explicitamente.

Temos então uma base de Hilbert 6-dimensional cujo reticulado é o conjunto de todos os vetores inteiros. Vamos obter um vetor inteiro no cone dessa base que não pode ser gerado pela combinação inteira de menos do que 7 vetores. Esse vetor, que prova que as conjecturas são falsas, é

$$
v=(9,13,13,13,13,13)
$$

gerado pela soma $z_{1}+3 z_{2}+5 z_{4}+2 z_{5}+z_{8}+5 z_{9}+3 z_{10}$.

Para mostrar que esse vetor não pode ser gerado pela combinação inteira não-negativa de menos do que 7 vetores, podemos considerar uma solução para o programa linear inteiro

$$
\begin{aligned}
\operatorname{minimizar} & \sum_{i=1}^{10} \mu_{i} \\
\text { sujeito a } & \sum_{i=1}^{10} \eta_{i} z_{i}=v \\
& 0 \leq \eta_{i} \leq 13 \mu_{i} \\
& 0 \leq \mu_{i} \leq 1 \\
& \eta_{i}, \mu_{i} \in \mathbb{Z}
\end{aligned}
$$

$\mathrm{O}$ valor de cada $\mu_{i}$ é 0 ou 1 . Mais precisamente, é zero se $\eta_{i}=0$ e é um se $\eta_{i}>0$. Portanto, o valor minimizado é precisamente a quantidade de coeficientes positivos dentre os $\eta_{i}$. Por outro lado, queremos que esses $\eta_{i}$ sejam coeficientes inteiros não-negativos de uma combinação dos vetores $z_{i}$ que gera $v$. Ademais, como todo $z_{i}$ é inteiro, não-negativo e não-nulo, segue que $\eta_{i} \leq 13$ para qualquer solução do problema, independentemente da segunda restrição.

Da resolução desse programa linear inteiro segue que a combinação dada inicialmente é uma solução ótima e, portanto, que são necessários no mínimo sete vetores para gerar $v$. Logo, as conjecturas são falsas para um cone com dimensão 6 . Na verdade, essa base pode ser utilizada para gerar toda uma família de contra-exemplos, como mostra o resultado a seguir.

Teorema 6.26. Para todo inteiro positivo $r$, existe uma base de Hilbert $\mathcal{H}$ tal que cone $(\mathcal{H})$ é pontudo, $\operatorname{dim}(\mathcal{H})=r$ e tal que algum vetor $v$ no cone inteiro de $\mathcal{H}$ é combinação inteira não-negativa de no mínimo $\lfloor 7 r / 6\rfloor$ vetores em $\mathcal{H}$.

Demonstração. Primeiramente, vamos provar a afirmação para o caso $r=6 k$, onde $k$ é um inteiro positivo. A prova é por indução em $k$, com a base $k=1$ sendo o contra-exemplo que vimos anteriormente. Suponha então que $k>1$ e que a afirmação vale para múltiplos menores.

Seja $\mathcal{H}_{6}$ um base que satisfaz a afirmação para o caso 6 e seja $\mathcal{H}_{6(k-1)}$ uma base que satisfaz a afirmação para o caso $6(k-1)$. Considere então o espaço $\mathbb{R}^{6 k}$ e tome $\mathcal{H}_{6}^{\prime}$ como sendo uma imersão de $\mathcal{H}_{6}$ no reticulado gerado pelos 6 primeiros vetores canônicos e $\mathcal{H}_{6(k-1)}^{\prime}$ como sendo uma imersão de $\mathcal{H}_{6(k-1)}$ no reticulado gerado pelos $6(k-1)$ outros vetores canônicos. 
Seja $\mathcal{H}_{6 k}:=\mathcal{H}_{6}^{\prime} \cup \mathcal{H}_{6(k-1)}^{\prime}$. Por construção, o reticulado gerado por $\mathcal{H}_{6 k}$ é $\mathbb{Z}^{6 k}$. Ademais, esse conjunto é uma base de Hilbert e todo vetor inteiro $v$ é combinação inteira não-negativa de no mínimo $\lfloor 7 \cdot 6 / 6\rfloor+\lfloor 7 \cdot 6(k-1) / 6\rfloor=7+7(k-1)=7 k=\lfloor 7 \cdot 6 k / 6\rfloor$ vetores.

Para o caso em que $r$ não é um múltiplo de 6 , temos $r=6(k-1)+l$, onde $k$ é um inteiro não-negativo e $0 \leq l \leq 5$. Nesse caso, cabe observar que o limitante inferior que queremos é

$$
\left\lfloor\frac{7 \cdot 6(k-1)}{6}+\frac{7 l}{6}\right\rfloor=\left\lfloor 7(k-1)+l+\frac{l}{6}\right\rfloor=7(k-1)+l
$$

Portanto, podemos utilizar a estratégia de criar uma base $\mathcal{H}_{6(k-1)}^{\prime} \cup \mathcal{H}_{l}^{\prime}$, mas não precisamos nos preocupar com as propriedades de $\mathcal{H}_{l}^{\prime}$ : conseguimos o limitante inferior desejado mesmo se $\mathcal{H}_{l}^{\prime}$ for uma base de Hilbert que satisfaz as conjecturas.

Portanto, podemos concluir que

Corolário 6.27. Todas as conjecturas são falsas para $\operatorname{dim}(\mathcal{H}) \geq 6$.

Demonstração. Temos $\lfloor 7 r / 6\rfloor=\lfloor r+r / 6\rfloor \geq r+1$ para todo $r \geq 6$.

O menor $r$ tal que um vetor no cone inteiro da base de Hilbert $\mathcal{H}$ é gerado por uma combinação inteira não-negativa de não mais do que $r$ vetores em $\mathcal{H}$ é o posto de Carathéodory de $\mathcal{H}$.

Ainda não se conhece nenhum contra-exemplo combinatório para as conjecturas. Bases de Hilbert combinatórias serão o foco do próximo capítulo. 


\section{Capítulo 7}

\section{Bases de Hilbert e combinatória}

Neste capítulo, vamos relacionar os resultados sobre bases de Hilbert que demonstramos nos capítulos anteriores a alguns resultados em otimização combinatória e teoria dos grafos. Nas três primeiras seções, essa relação é estabelecida através de sistemas totalmente dual integrais. Nas duas últimas seções, consideramos conjuntos gerados por vetores de incidência de objetos combinatórios. O posto de Carathéodory de certas bases de Hilbert também é considerado.

Priorizou-se aqui uma abordagem mais didática: tentamos apresentar os principais teoremas do capítulo como conclusões de seqüências naturais de passos. O leitor mais ansioso pode ir direto para o final da seção caso queira saber antecipadamente aonde estamos querendo chegar.

\subsection{Orientações $k$-conexas de grafos}

Seja $G=(V, E)$ um grafo e sejam $e_{1}, \ldots, e_{|E|}$ as arestas desse grafo. Uma orientação de $G$ é um grafo dirigido $D=(N, A)$ tal que $N=V$ e $A=\left\{\left(u_{1}, v_{1}\right), \ldots,\left(u_{|E|}, v_{|E|}\right)\right\}$, onde $e_{i}=\left\{u_{i}, v_{i}\right\}$ para todo $i$. Dizemos que o arco $\left(u_{i}, v_{i}\right)$ é correspondente a $e_{i}$. Ao longo desta seção, vamos sempre supor que $D$ é uma orientação de $G$. A figura 7.1 mostra uma orientação de um grafo.
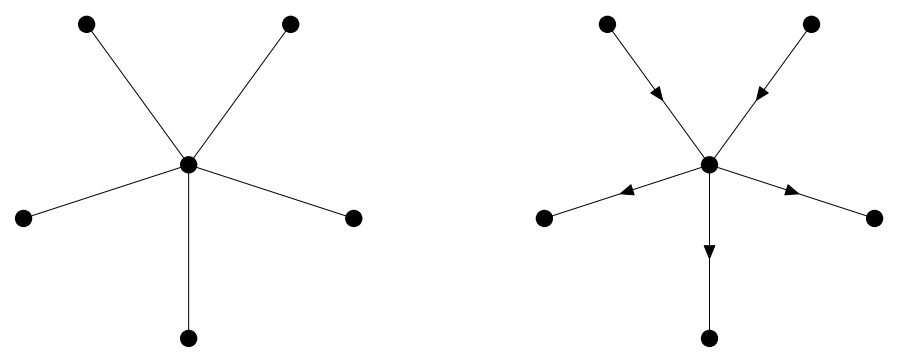

Figura 7.1: exemplo de grafo e uma de suas orientações.

Tanto para grafos como para grafos dirigidos, podemos considerar o conceito de $k$-conexidade. Seja $k$ um inteiro não-negativo. Dizemos que $G$ é um grafo $k$-conexo se

para todo subconjunto próprio não-vazio $V^{\prime}$ de $V$, temos $\left|\delta\left(V^{\prime}\right)\right| \geq k$.

Analogamente, dizemos que $D$ é um grafo dirigido $k$-conexo se

para todo subconjunto próprio não-vazio $N^{\prime}$ de $N$, temos $\left|\underline{\delta_{(}}\left(N^{\prime}\right)\right| \geq k$. 
Na verdade, essa definição poderia considerar tanto $\delta_{\rightarrow}\left(N^{\prime}\right)$ como $\underset{\&}{\delta}\left(N^{\prime}\right)$. Se $D$ é $k$-conexo e $N^{\prime}$ é um subconjunto próprio não-vazio de $N$, então $\left|\delta\left(N^{\prime}\right)\right| \geq k$, pois nesse caso $N \backslash N^{\prime}$ também é um subconjunto próprio não-vazio de $N$ e $\underline{\delta}\left(N \backslash N^{\prime}\right)=\underline{\delta}\left(N^{\prime}\right)$.

Sabemos que $\left|\delta_{(}\left(N^{\prime}\right)\right| \geq k$ e $\left|\delta\left(N^{\prime}\right)\right| \geq k$ para todo $N^{\prime}$ tal que $\emptyset \subset N^{\prime} \subset N$ somente se $\left|\delta\left(V^{\prime}\right)\right| \geq 2 k$ para todo $V^{\prime}$ tal que $\emptyset \subset V^{\prime} \subset V$. Logo, a orientação de um grafo é $k$-conexa somente se tal grafo é $2 k$-conexo. Essa condição, no entanto, não é suficiente para que qualquer orientação seja k-conexa. A figura 7.2 mostra duas orientações de um grafo 2-conexo, sendo apenas uma delas 1-conexa.
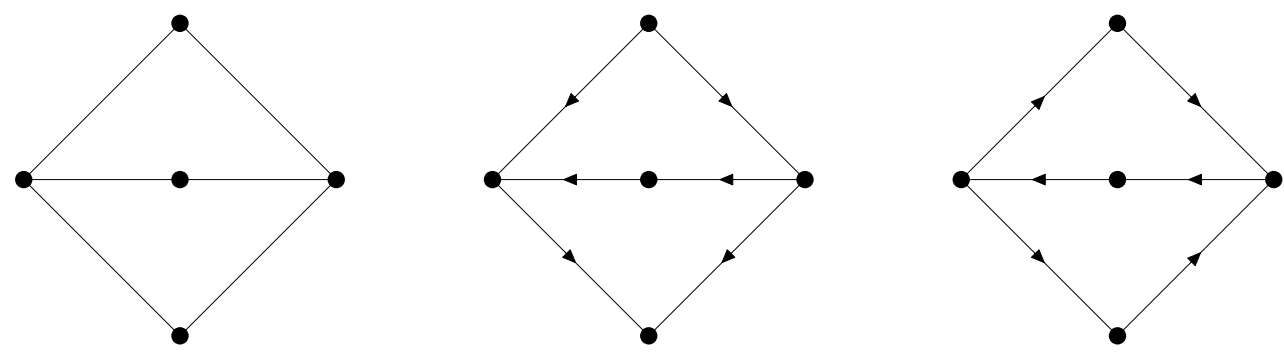

Figura 7.2: Exemplo de grafo 2-conexo e de duas orientações desse grafo. A orientação à direita é 1-conexa, mas a outra não.

Vamos demonstrar, no entanto, que a condição é suficiente para que uma orientação $k$-conexa de $G$ exista, mesmo que $D$ não seja tal orientação. Sabemos pela definição de orientação que, para cada aresta $\{u, v\}$ de $G$, o arco correspondente a essa aresta em uma orientação do grafo é $(u, v)$ ou $(v, u)$. Portanto, se $D$ não é $k$-conexo mas existe alguma orientação $k$-conexa de $G$, então existe algum subconjunto $X$ de arcos de $D$ tal que $(N,(A \backslash X) \cup Y)$ é uma orientação $k$-conexa de $G$, onde $Y$ é o conjunto dos inversos dos arcos em $X$. A figura 7.3 mostra um exemplo dessa transformação.
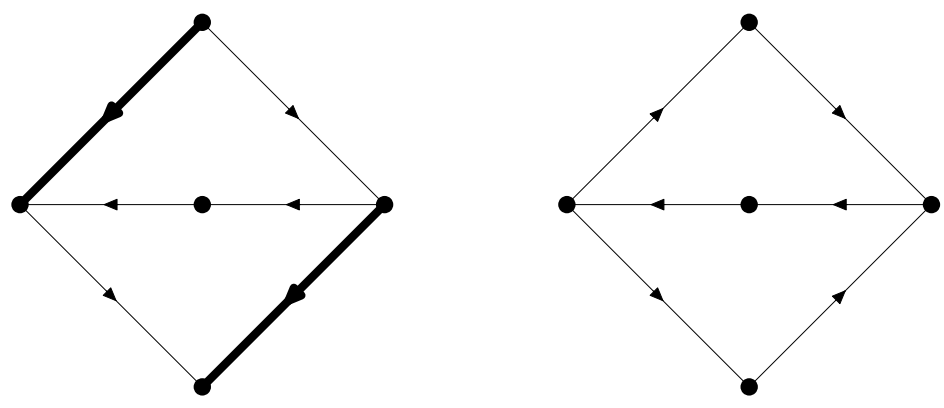

Figura 7.3: As duas orientações da figura 7.2. Substituindo todos os arcos acentuados por seus inversos, obtemos a orientação $k$-conexa.

Portanto, pela definição de $k$-conexidade, gostaríamos de obter um conjunto $X \subseteq A$ tal que

$$
\left|\delta_{\rightarrow}\left(N^{\prime}\right)\right|+\left|\underline{\underline{\delta}}\left(N^{\prime}\right) \cap X\right|-\left|\underline{\delta}_{(}\left(N^{\prime}\right) \cap X\right| \geq k \text { para todo } N^{\prime} \text { tal que } \emptyset \subset N^{\prime} \subset N .
$$

Vamos então verificar como podemos reformular esse problema em termos de álgebra linear, de modo a podermos aplicar os resultados sobre bases de Hilbert vistos nos capítulos anteriores.

Sendo $X$ um subconjunto, uma representação algébrica natural de $X$ é seu vetor de incidência. Pela definição de vetor de incidência, estamos interessados em verificar se existe um subconjunto dos arcos de $D$ tal que, se $x$ é o vetor de incidência desse subconjunto, então

$$
x\left(\underline{\delta}\left(N^{\prime}\right)\right)-x\left(\underline{\delta}_{(}\left(N^{\prime}\right)\right) \geq k-\left|\underline{\delta}_{(}\left(N^{\prime}\right)\right| \text { para todo } N^{\prime} \text { tal que } \emptyset \subset N^{\prime} \subset N .
$$


Os vetores que satisfazem essa condição, portanto, podem ser naturalmente representados por um poliedro. Seja $A$ uma matriz cujas colunas são indexadas pelos arcos de $D$ e cujas linhas são indexadas pelos subconjuntos próprios não-vazios de $N$. Seja $b$ um vetor também indexado por esses subconjuntos. Suponha que, se $N^{\prime}$ é um subconjunto próprio não-vazio de $N$ e $a$ é a linha de $A$ associada a esse subconjunto, então $a$ associa 1 a todo arco de $\delta\left(N^{\prime}\right)$, associa -1 a todo arco de $\underline{\delta}\left(N^{\prime}\right)$ e associa 0 aos arcos restantes. Suponha também que $b\left(N^{\prime}\right)=k-\left|\delta\left(N^{\prime}\right)\right|$ para todo $N^{\prime}$. Estamos interessados em demonstrar que existe um vetor de incidência $x$ tal que $A x \geq b$. Pela definição de vetor de incidência, tal vetor só existe se o poliedro

$$
\mathcal{P}:=\{x:-A x \leq-b,-I x \leq 0, I x \leq 1\}
$$

contém pelo menos um vetor inteiro. Podemos verificar que, se a integralidade não fosse exigida, poderíamos simplesmente considerar $x:=(1 / 2, \ldots, 1 / 2)$, pois nesse caso

$$
\begin{aligned}
& x\left(\underline{\delta}\left(N^{\prime}\right)\right)-x\left(\delta_{\rightarrow}\left(N^{\prime}\right)\right)=\frac{1}{2}\left|\delta_{L}\left(N^{\prime}\right)\right|-\frac{1}{2}\left|\delta_{\rightarrow}\left(N^{\prime}\right)\right| \\
& =\frac{1}{2}\left|\underline{\phi}\left(N^{\prime}\right)\right|-\left(\left|\underline{\delta}_{\rightarrow}\left(N^{\prime}\right)\right|-\frac{1}{2}\left|\delta_{\rightarrow}\left(N^{\prime}\right)\right|\right) \\
& =\frac{1}{2}\left(\left|\delta_{L}\left(N^{\prime}\right)\right|+\left|\underline{\delta}_{\rightarrow}\left(N^{\prime}\right)\right|\right)-\left|\delta_{\rightarrow}\left(N^{\prime}\right)\right| \\
& \geq \frac{1}{2} \cdot 2 k-\left|\underline{\delta}\left(N^{\prime}\right)\right| \\
& =k-\left|\underline{\delta}_{\rightarrow}\left(N^{\prime}\right)\right|
\end{aligned}
$$

para todo $N^{\prime}$ tal que $\emptyset \subset N^{\prime} \subset N$. Na verdade, esse vetor é particularmente interessante mesmo se não desconsiderarmos a integralidade, pois ele demonstra que $\mathcal{P}$ é não-vazio. Portanto, podemos concluir que $\mathcal{P}$ contém um vetor inteiro se demonstrarmos que ele é um poliedro inteiro.

Sabemos pelo teorema 5.4 que $\mathcal{P}$ é um poliedro inteiro se o sistema $-A x \leq-b,-I x \leq 0, I x \leq 1$ é totalmente dual integral. Para demonstrar que esse sistema é totalmente dual integral, precisamos demonstrar que, para todo vetor inteiro $c$, existe uma solução inteira para o programa linear

$$
\min \left\{y b^{\prime}: y A^{\prime}=c ; y \geq 0\right\}, \text { onde } b^{\prime}=\left(\begin{array}{r}
-b \\
0 \\
1
\end{array}\right) \text { e } A^{\prime}=\left[\begin{array}{r}
-A \\
-I \\
I
\end{array}\right]
$$

se esse programa admite solução. Pelo corolário 2.12, precisamos apenas verificar que

existe uma solução para o programa linear (7.2) tal que c pertence ao cone inteiro das linhas de $A^{\prime}$ correspondentes aos elementos positivos dessa solução.

Pela definição de sistema gerador de Hilbert, uma condição suficiente para essa propriedade é: existe uma solução para o programa linear (7.2) tal que as linhas de $A^{\prime}$ correspondentes aos elementos positivos dessa solução formam um sistema gerador de Hilbert.

Em particular, é suficiente que um caso específico dessa condição seja válido: existe uma solução para o programa linear (7.2) tal que as linhas de $A^{\prime}$ correspondentes aos elementos positivos dessa solução induzem uma submatriz totalmente unimodular. 
Finalmente, pelo lema 4.14, podemos verificar que um conjunto de linhas de $A^{\prime}$ induz uma submatriz totalmente unimodular se e somente se as linhas desse subconjunto que pertencem a $A$ induzem uma submatriz totalmente unimodular. Portanto, estamos interessados em demonstrar que

existe uma solução para o programa linear (7.2) tal que as linhas de A correspondentes aos elementos positivos dessa solução induzem uma submatriz totalmente unimodular.

Para toda solução $y$ do programa (7.2), vamos denotar por $\mathcal{N}(y)$ o conjunto $\left\{N^{\prime}: y\left(N^{\prime}\right)>0\right\}$. Queremos demonstrar que existe uma solução $y^{\prime}$ tal que as linhas de $A$ correspondentes a $\mathcal{N}\left(y^{\prime}\right)$ induzem uma submatriz totalmente unimodular. Essa solução $y^{\prime}$ pode ser obtida se utilizarmos uma estratégia de descruzamento. Para dado $y$, dizemos que $\mathcal{N}(y)$ é uma família livre de cruzamentos se, para quaisquer $N_{1}^{\prime}, N_{2}^{\prime} \in \mathcal{N}(y)$, temos que

$$
N_{1}^{\prime} \subseteq N_{2}^{\prime} \quad \text { ou } \quad N_{2}^{\prime} \subseteq N_{1}^{\prime} \quad \text { ou } \quad N_{1}^{\prime} \cap N_{2}^{\prime}=\emptyset \quad \text { ou } \quad N_{1}^{\prime} \cup N_{2}^{\prime}=N .
$$

Segundo um conhecido teorema de EDmonds E Giles [1977], as linhas de $A$ correspondentes a $\mathcal{N}(y)$ para um dado $y$ induzem uma submatriz totalmente unimodular se esse conjunto é uma família livre de cruzamentos. Considere então uma solução $y^{\prime}$ de (7.2) que maximiza a soma

$$
\varphi\left(y^{\prime}\right)=\sum_{\emptyset \subset N^{\prime} \subset N}\left|N^{\prime}\right|^{2} y^{\prime}\left(N^{\prime}\right) .
$$

Consideremos o conjunto $\mathcal{N}\left(y^{\prime}\right)$ correspondente. Suponha que existem $N_{1}^{\prime}, N_{2}^{\prime} \in \mathcal{N}\left(y^{\prime}\right)$ tais que

$$
N_{1}^{\prime} \nsubseteq N_{2}^{\prime} \quad \text { e } \quad N_{2}^{\prime} \nsubseteq N_{1}^{\prime} \quad \text { e } \quad N_{1}^{\prime} \cap N_{2}^{\prime} \neq \emptyset \quad \text { e } \quad N_{1}^{\prime} \cup N_{2}^{\prime} \neq N .
$$

Nesse caso, sabemos que $N_{1}^{\prime} \cap N_{2}^{\prime}$ e $N_{1}^{\prime} \cup N_{2}^{\prime}$ também são subconjuntos próprios não-vazios de $N$. Seja $\varepsilon:=\min \left\{y^{\prime}\left(N_{1}^{\prime}\right), y^{\prime}\left(N_{2}^{\prime}\right)\right\}$ e seja $y^{\prime \prime}$ um vetor definido como a seguir:

$$
y^{\prime \prime}\left(N^{\prime}\right)= \begin{cases}y^{\prime}\left(N^{\prime}\right)-\varepsilon & \text { se } N^{\prime}=N_{1}^{\prime} \text { ou } N^{\prime}=N_{2}^{\prime} ; \\ y^{\prime}\left(N^{\prime}\right)+\varepsilon & \text { se } N^{\prime}=N_{1}^{\prime} \cap N_{2}^{\prime} \text { ou } N^{\prime}=N_{1}^{\prime} \cup N_{2}^{\prime} ; \\ y^{\prime}\left(N^{\prime}\right) & \text { caso contrário. }\end{cases}
$$

A partir dessa definição, podemos verificar que

(i) $y^{\prime \prime} A^{\prime}=y^{\prime} A^{\prime}$;

(ii) $y^{\prime \prime} \geq 0$;

(iii) $y^{\prime \prime} b=y^{\prime} b-\varepsilon\left(\left|\underline{\delta_{(}}\left(N_{1}^{\prime}\right)\right|+\left|\underline{\delta_{(}}\left(N_{2}^{\prime}\right)\right|\right)+\varepsilon\left(\left|\underline{\delta_{(}}\left(N_{1}^{\prime} \cap N_{2}^{\prime}\right)\right|+\left|\underline{\delta}_{(}\left(N_{1}^{\prime} \cup N_{2}^{\prime}\right)\right|\right)$;

(iv) $\varphi\left(y^{\prime \prime}\right)=\varphi\left(y^{\prime}\right)-\varepsilon\left(\left|N_{1}^{\prime}\right|^{2}+\left|N_{2}^{\prime}\right|^{2}\right)+\varepsilon\left(\left|N_{1}^{\prime} \cap N_{2}^{\prime}\right|^{2}+\left|N_{1}^{\prime} \cup N_{2}^{\prime}\right|^{2}\right)$.

Sabemos pelas propriedades (i) e (ii) que o vetor $y^{\prime \prime}$ pertence ao poliedro do programa linear (7.2). Vamos então considerar a propriedade (iii). A figura 7.4 mostra todos os tipos de arco que podem entrar nos quatro conjuntos considerados. Podemos então verificar a partir dessa figura que

$$
\left|\delta_{\rightarrow}\left(N_{1}^{\prime}\right)\right|+\left|\underline{\delta}_{(}\left(N_{2}^{\prime}\right)\right| \geq\left|\underline{\delta}_{\rightarrow}\left(N_{1}^{\prime} \cap N_{2}^{\prime}\right)\right|+\left|\underline{\delta}_{(}\left(N_{1}^{\prime} \cup N_{2}^{\prime}\right)\right| .
$$

Logo, pelas propriedades (i), (ii) e (iii) temos que $y^{\prime \prime}$ também é solução para (7.2). Por fim, vamos considerar a propriedade (iv). Como $\left|N_{1}^{\prime}\right|+\left|N_{2}^{\prime}\right|=\left|N_{1}^{\prime} \cap N_{2}^{\prime}\right|+\left|N_{1}^{\prime} \cup N_{2}^{\prime}\right|$, podemos verificar que

$$
\left|N_{1}^{\prime}\right|^{2}+\left|N_{2}^{\prime}\right|^{2}<\left|N_{1}^{\prime} \cap N_{2}^{\prime}\right|^{2}+\left|N_{1}^{\prime} \cup N_{2}^{\prime}\right|^{2} .
$$




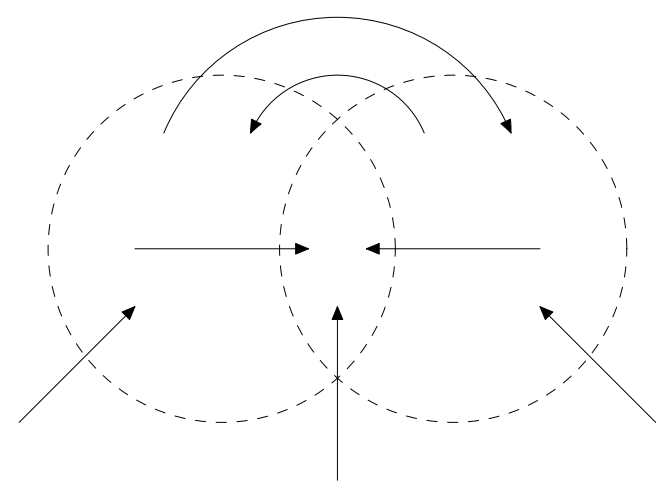

Figura 7.4: diagrama de Venn de dois subconjuntos de vértices e os tipos de arco que podem entrar em cada um desses subconjuntos, em sua união e em sua intersecção.

Como essa desigualdade contradiz a hipótese de que a soma $\varphi\left(y^{\prime}\right)$ é máxima, podemos concluir que $\mathcal{N}\left(y^{\prime}\right)$ é uma família livre de cruzamentos. Recapitulando os argumentos desta seção, temos o teorema da orientação, que foi demonstrado originalmente por NASH-WILLIAMS [1969]:

Teorema 7.1. Seja $G$ um grafo e seja $k$ um inteiro não-negativo. Se $G$ é $2 k$-conexo, então existe uma orientação k-conexa de $G$.

Demonstração. Seja $D=(N, A)$ uma orientação de $G$ e considere o poliedro (7.1). Sabemos que $G$ admite uma orientação $k$-conexa se e somente se esse poliedro contém um vetor inteiro. Seja $c$ um vetor inteiro tal que o programa linear (7.2) admite solução e seja $y^{\prime}$ uma solução desse programa tal que $\varphi\left(y^{\prime}\right)$ é máximo. Pela maximalidade de $\varphi\left(y^{\prime}\right)$, sabemos que $\mathcal{N}\left(y^{\prime}\right)$ é uma família livre de cruzamentos. Logo, as linhas da matriz $C$ correspondentes a esse conjunto induzem uma submatriz totalmente unimodular. Pelo lema 4.14, as linhas de $C^{\prime}$ correspondentes aos elementos positivos de $y^{\prime}$ também induzem uma submatriz totalmente unimodular e, portanto, formam uma base de Hilbert. A partir desse fato, podemos verificar que $c$ pertence ao cone inteiro desse conjunto de linhas de $C^{\prime}$. Logo, pelo corolário 2.12, sabemos que (7.2) admite solução inteira. Portanto, pelo teorema 5.4, podemos verificar que (7.1) é um poliedro inteiro. Sabemos também que esse poliedro não é vazio, pois contém $(1 / 2, \ldots, 1 / 2)$. Podemos concluir que ele contém um vetor inteiro.

A demonstração poliédrica que descrevemos nesta seção foi apresentada por FRANK [1980] e, posteriormente, por FRANK E TARDOs [1984]. Embora estivéssemos interessados em demonstrar apenas que um certo poliedro contém um vetor inteiro, a estratégia de descruzamento que foi utilizada nos permitiu demonstrar um fato muito mais forte: podemos definir sobre esse poliedro uma versão inteira do teorema da dualidade. Na seção a seguir, vamos apresentar um exemplo de como podemos utilizar a mesma estratégia para demonstrarmos relações min-max em otimização combinatória.

\section{$7.2 \quad r$-arborescências e $r$-cortes}

Seja $D=(N, A)$ um grafo dirigido e seja $r$ um vértice desse grafo. Dizemos que uma árvore geradora dirigida $A^{\prime} \subseteq A$ é uma $r$-arborescência de $D$ se $\left|\underline{\rightarrow}(v) \cap A^{\prime}\right|=1$ para todo $v \in N \backslash\{r\}$. Dizemos que um corte $\underline{\delta}_{\rightarrow}\left(N^{\prime}\right)$ é um $r$-corte de $D$ se $\emptyset \subset N^{\prime} \subseteq N \backslash\{r\}$. A figura 7.5 mostra um exemplo de $r$-arborescência e um exemplo de $r$-corte. 

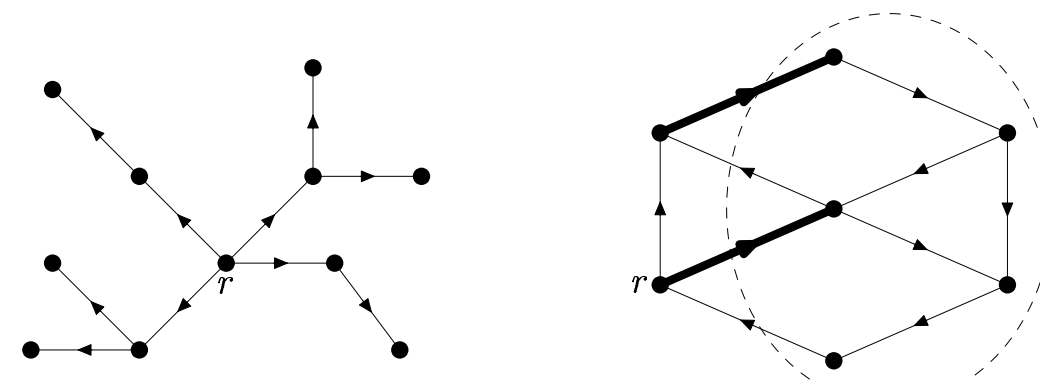

Figura 7.5: À esquerda, um exemplo de $r$-arborescência. À direita, um exemplo de $r$-corte. O conjunto de vértices correspondente ao $r$-corte é indicado pelo círculo.

A partir da definição de $r$-arborescência, podemos verificar que

se $A^{\prime}$ é uma r-arborescência de $D$ e $v$ é um vértice de $D$, então existe um e apenas um caminho dirigido de $r$ a $v$ no grafo dirigido $\left(N, A^{\prime}\right)$.

Portanto, se $D$ contém pelo menos uma $r$-arborescência, podemos verificar a partir desse fato que

para todo subconjunto $A^{\prime}$ de $A$, temos que $A^{\prime}$ é uma $r$-arborescência de $D$ se e somente se $A^{\prime}$ é um conjunto minimal de arcos que intersecta todos os r-cortes de $D$.

Vamos então supor nesta seção que o grafo considerado contém pelo menos uma $r$-arborescência. Sob essa hipótese, podemos verificar que todos os $r$-cortes são não-vazios.

Temos então uma maneira natural de reformular a definição de $r$-arborescência em termos de álgebra linear. Se $x$ é o vetor de incidência de um conjunto de arcos, então podemos verificar que esse subconjunto é uma $r$-arborescência se e somente se

(i) $x\left(\underline{\delta}\left(N^{\prime}\right)\right) \geq 1$ para todo $N^{\prime}$ tal que $\emptyset \subset N^{\prime} \subseteq N \backslash\{r\}$;

(ii) $x(A)$ é mínimo.

Seja $C$ uma matriz cujas colunas são indexadas pelos arcos de $D$ e cujas linhas são indexadas pelos subconjuntos não-vazios de $N$ que não contêm $r$. Suponha que, se $N^{\prime}$ é tal que $\emptyset \subset N^{\prime} \subseteq N \backslash\{r\}$, a sua linha correspondente em $C$ é o vetor de incidência do $r$-corte $\underline{\delta}_{f}\left(N^{\prime}\right)$.

Podemos verificar que $x$ é o vetor de incidência de uma $r$-arborescência de $D$ se e somente se $x$ é inteiro, $C x \geq 1, x \geq 0, x \leq 1$ e o valor de $1 x$ é mínimo. Podemos verificar também que a minimalidade de $1 x$ faz com que a restrição $x \leq 1$ seja desnecessária. As $r$-arborescências de $D$ são, portanto, representadas pelas soluções do programa inteiro

$$
\min \{1 x:-C x \leq-1,-I x \leq 0 ; x \text { inteiro }\} \text {. }
$$

Pelo mesmo argumento podemos verificar que, para todo vetor inteiro positivo $c$, as soluções de

$$
\min \{c x:-C x \leq-1,-I x \leq 0 ; x \text { inteiro }\}
$$

são vetores de incidência de $r$-arborescências. $\mathrm{O}$ vetor $c$ pode ser interpretado como sendo uma função que atribui custos aos arcos de $D$. Para todo conjunto de $\operatorname{arcos} A^{\prime}$, temos que $c\left(A^{\prime}\right)$ é o custo atribuído por $c$ a $A^{\prime}$. As soluções de (7.4) representam, portanto, as r-arborescências de custo mínimo em relação à função de custos $c$. 
Vamos então considerar o dual da relaxação linear desse programa. Desconsiderando a restrição de integridade em (7.4), obtemos um programa linear cujas soluções são as mesmas do programa

$$
\max \{-c x:-C x \leq-1,-I x \leq 0\} .
$$

O dual desse programa de maximização é

$$
\min \left\{y b^{\prime}: y C^{\prime}=-c ; y \geq 0\right\}, \text { onde } b^{\prime}=\left(\begin{array}{r}
-1 \\
0
\end{array}\right) \text { e } C^{\prime}=\left[\begin{array}{l}
-C \\
-I
\end{array}\right] .
$$

Novamente como conseqüência de um teorema de EDMONDS E GiLEs [1977], temos para todo $c$ inteiro que, se $y$ é uma solução do programa linear (7.5) e $\mathcal{N}(y)$ é uma família livre de cruzamentos, então as linhas de $C$ correspondentes a essa família induzem uma submatriz totalmente unimodular. De maneira completamente análoga à da demonstração da seção anterior, podemos verificar que, se $y^{\prime}$ é uma solução de (7.5) que maximiza $\varphi\left(y^{\prime}\right)$, então $\mathcal{N}\left(y^{\prime}\right)$ é uma família livre de cruzamentos. Podemos então concluir que o sistema $-C x \leq-1,-I x \leq 0$ é totalmente dual integral.

Como $C$ é uma matriz inteira, podemos verificar também que os programas lineares inteiros

$$
\min \left\{y b^{\prime}: y C^{\prime}=-c ; y \geq 0 ; y \text { inteiro }\right\} \quad \text { e } \quad \min \{y(-1): y(-C) \geq-c ; y \geq 0 ; y \text { inteiro }\}
$$

são equivalentes. Ademais, temos que as soluções do segundo programa são as mesmas de

$$
\max \{y 1: y C \leq c ; y \geq 0 ; y \text { é inteiro }\}
$$

Podemos então concluir que, para todo vetor inteiro positivo $c$, temos a dualidade inteira

$$
\min \{c x:-C x \leq-1,-I x \leq 0 ; x \text { inteiro }\}=\max \{y 1: y C \leq c ; y \geq 0 ; y \text { inteiro }\} \text {. }
$$

Da mesma maneira como as soluções de (7.4) são $r$-arborescências de $D$, vamos considerar uma interpretação combinatória para as soluções de (7.6) através de uma extensão natural do conceito de vetor de incidência. Para todo vetor inteiro não-negativo $y$ indexado pelos subconjuntos não-vazios de $N \backslash\{r\}$, vamos denotar por $\mathcal{C}(y)$ um multiconjunto de $r$-cortes de $D$ tal que, se $\emptyset \subset N^{\prime} \subseteq N^{\prime} \backslash\{r\}$, então o conjunto $\mathcal{C}(y)$ contém $y\left(N^{\prime}\right)$ cópias de $\underline{\delta}_{(}\left(N^{\prime}\right)$.

Podemos verificar que, se $y$ é uma solução de (7.6), então o vetor $c$ associa a cada arco um limitante superior para o número de elementos de $\mathcal{C}(y)$ aos quais esse arco pertence. Dizemos nesse caso que o conjunto $\mathcal{C}(y)$ é uma $c$-cobertura por cortes dos arcos de $D$. Podemos então formular a relação min-max conhecida como teorema da arborescência ótima de FULKERSON [1974]:

Teorema 7.2. Seja $D=(N, A)$ um grafo dirigido, seja $r$ um vértice desse grafo e seja c uma função que associa custos inteiros positivos aos arcos do grafo. Se existe pelo menos uma r-arborescência em $D$, então o custo mínimo em relação a c de uma r-arborescência de $D$ é igual à cardinalidade máxima de uma c-cobertura por cortes dos arcos de $D$.

A partir da total dual integralidade do sistema considerado na demonstração desse teorema, podemos também obter um resultado relativo ao posto de Carathéodory. Como estamos supondo que cada $r$-corte do grafo $D$ contém pelo menos um arco, podemos verificar que $-C x<-1,-I x<0$ para todo $x>1$. Logo, sabemos que o poliedro definido por $-C x \leq 1,-I x \leq 0$ tem dimensão plena. Portanto, podemos demonstrar o teorema a seguir através do corolário 6.2. 
Teorema 7.3. Seja $D=(N, A)$ um grafo dirigido, seja $r$ um vértice desse grafo e seja c uma função que associa custos inteiros positivos aos arcos do grafo. Se existe pelo menos uma r-arborescência em $D$, então existe uma c-cobertura por cortes $\mathcal{C}$ dos arcos de $D$ tal que a cardinalidade de $\mathcal{C} e ́$ máxima e $\mathcal{C}$ contém não mais do que $2|A|-1$ tipos de corte.

Se as conjecturas de Sebő fossem verdadeiras, então o limitante superior descrito nesse teorema seria $|A|$. Na seção a seguir, apresentaremos um sistema totalmente dual integral para o qual todas as conjecturas são válidas e, portanto, podemos obter o melhor posto de Carathéodory possível.

\subsection{Cliques de grafos perfeitos}

Seja $G=(V, E)$ um grafo simples e seja $V^{\prime}$ um conjunto de vértices desse grafo. Dizemos que $V^{\prime}$ é um clique do grafo se o subgrafo de $G$ induzido por esse subconjunto é completo e dizemos que $V^{\prime}$ é um conjunto estável do grafo se o subgrafo de $G$ induzido por esse subconjunto não contém nenhuma aresta. Podemos então verificar que

um conjunto de vértices de $G$ é um conjunto estável se e somente se cada clique do grafo contém no máximo um vértice desse subconjunto.

Logo, podemos obter para os conjuntos estáveis do grafo uma descrição poliédrica similar à caracterização das $r$-arborescências apresentada na seção anterior. Seja $K$ uma matriz cujas linhas são indexadas pelos cliques de $G$ e cujas colunas são indexadas pelos vértices de $G$. Suponha que cada linha de $K$ é o vetor de incidência do clique correspondente a essa linha.

Podemos verificar que $x$ é vetor de incidência de um conjunto estável se e somente se $x$ é inteiro, $K x \leq 1$ e $0 \leq x \leq 1$. Como pela definição de clique temos que $\{v\}$ é um clique para todo $v \in V$, podemos verificar também que $K x \leq 1$ somente se $x \leq 1$. Podemos então desconsiderar a última restrição e concluir que os vetores de incidência dos conjuntos estáveis são os vetores inteiros em

$$
\mathcal{P}=\{x: K x \leq 1,-I x \leq 0\} .
$$

Sabemos então que, para todo vetor $c$ que atribui custos aos vértices, as soluções do programa

$$
\max \{c x: A x \leq b ; x \text { é inteiro }\}, \text { onde } A=\left[\begin{array}{c}
K \\
-I
\end{array}\right] \text { e } b=\left(\begin{array}{l}
1 \\
0
\end{array}\right)
$$

são os vetores de incidência dos conjuntos estáveis de custo máximo. Ademais, podemos verificar também que o programa inteiro associado ao dual da relaxação linear de (7.7) é equivalente a

$$
\min \{y 1: y K \geq c ; y \geq 0 ; y \text { inteiro }\}
$$

Vamos então considerar uma interpretação combinatória para as soluções de (7.8). Seja $y$ um vetor inteiro não-negativo indexado pelos cliques de $G$. Analogamente às definições apresentadas na seção anterior, vamos denotar por $\mathcal{K}(y)$ um multiconjunto de cliques tal que, para todo clique $V^{\prime}$, temos que $\mathcal{K}(y)$ contém $y\left(V^{\prime}\right)$ cópias de $V^{\prime}$. Podemos então verificar que, se $y$ é inteiro, $y K \geq c$ e $y \geq 0$, então todo vértice $v$ do grafo pertence a pelo menos $c(v)$ cliques de $\mathcal{K}(y)$. Dizemos nesse caso que $\mathcal{K}(y)$ é uma $c$-cobertura por cliques dos vértices de $G$. Se $y$ é uma solução do programa (7.8), temos que $\mathcal{K}(y)$ é uma $c$-cobertura por cliques de cardinalidade minima.

Se o sistema $A x \leq b$ é totalmente dual integral, então temos uma relação min-max: para todo vetor inteiro $c$ que atribui custos aos arcos de $G$, o custo máximo de um conjunto estável é igual à 
cardinalidade mínima de uma $c$-cobertura por cliques dos vértices de $G$, supondo que pelo menos uma $c$-cobertura exista. LovÁsz [1972] demonstrou que esse sistema é totalmente dual integral para o caso particular em que $G$ é um grafo perfeito. Dizemos que um grafo é perfeito se para cada um de seus subgrafos induzidos temos que a cardinalidade máxima de um clique é igual à cardinalidade mínima de uma partição do conjunto de vértices em conjuntos estáveis. Vamos então supor ao longo desta seção que o grafo $G$ considerado é perfeito.

De maneira análoga à da seção anterior, podemos obter a partir da total dual integralidade do sistema um limitante superior para o menor número possível de tipos de clique em uma $c$-cobertura de cardinalidade mínima. Podemos verificar que $K x<1,-I x<0$ se $0<x<1 /|V|$. Logo, o poliedro $\mathcal{P}$ tem dimensão plena. Portanto, pelo corolário 6.2, concluímos que existe uma $c$-cobertura de cardinalidade mínima que contém não mais que $2|V|-1$ tipos de clique.

Vamos demonstrar a seguir que esse limitante superior pode ser melhorado para $|V|$. Essa demonstração é baseada em argumentos de Cook, Fonlupt E Schrijver [1986] e SeBó [1990]. Vamos utilizar o fato de que $\mathcal{P}$ é um tipo particular de poliedro. Como as colunas de $-I$ são linearmente independentes, sabemos que $A x=0$ somente se $x=0$. Ademais, podemos verificar que

- se $-I x \leq 0$, então $K x \geq 0$, pois a matriz $K$ é não-negativa por definição;

- se $K x \leq 1$, então $-I x \geq-1$, pois $\{v\}$ é um clique para todo vértice $v$.

Portanto, se $x$ pertence a $\mathcal{P}$, então $0 \leq K x \leq 1$ e $-1 \leq-I x \leq 0$. Podemos concluir então que

$\mathcal{P}$ é um poliedro limitado e $A x \geq b-1$ para todo $x \in \mathcal{P}$.

Devido a essa propriedade, o lema a seguir é particularmente interessante no presente contexto.

Lema 7.4. Seja $\mathcal{P}=\{x: A x \leq b\}$ um poliedro inteiro e limitado e seja c um vetor inteiro. Suponha que $A x=0$ somente se $x=0$ e que o programa linear

$$
\max \{c x: A x \leq b\}
$$

admite solução. Seja $\mathcal{F}$ uma face de $\mathcal{P}$ formada pelas soluções desse programa e seja ax $\leq \beta$ uma restrição de $A x \leq b$ ativa em $\mathcal{F}$. Se ax $\geq \beta-1$ para todo $x \in \mathcal{P}$, então existe uma solução y para $o$ programa linear dual

$$
\min \{y b: y A=c ; y \geq 0\}
$$

tal que o elemento de y correspondente à restrição ax $\leq \beta$ é maior ou igual a 1.

Demonstração. Seja $x^{*}$ uma solução de (7.9) e seja $y^{*}$ uma solução de (7.10). Para todo $i$, vamos denotar por $a_{i}$ a $i$-ésima linha de $A$, por $\beta_{i}$ o $i$-ésimo elemento de $b$ e por $\lambda_{i}^{*}$ o $i$-ésimo elemento de $y^{*}$. Seja $j$ o índice tal que $a_{j}=a$ e $\beta_{j}=\beta$. Como $a x \leq \beta$ é uma restrição ativa em $\mathcal{F}$, sabemos que $a_{j} x^{*}=\beta_{j}$. Podemos supor sem perda de generalidade que $x^{*}$ é um vetor inteiro, pois $\mathcal{P}$ é um poliedro inteiro. Podemos supor também sem perda de generalidade que $\lambda_{j}^{*}$ é máximo. A partir dessa maximalidade e do corolário 2.11, podemos verificar que $\lambda_{j}^{*}>0$.

Seja $c^{\prime}:=c-\lambda_{j}^{*} a_{j}$. Substituindo $c$ por $c^{\prime}$ em (7.9), podemos definir um outro programa linear:

$$
\max \left\{c^{\prime} x: A x \leq b\right\}=\max \left\{\left(c-\lambda_{j}^{*} a_{j}\right) x: A x \leq b\right\} .
$$

Analogamente, substituindo $c$ por $c^{\prime}$ em (7.10), podemos definir o programa linear dual a (7.11):

$$
\min \left\{y b: y A=c^{\prime} ; y \geq 0\right\}=\min \left\{y b: y A=c-\lambda_{j}^{*} a_{j} ; y \geq 0\right\}
$$


Seja $\lambda_{j}^{\prime}:=0$, seja $\lambda_{i}^{\prime}:=\lambda_{i}^{*}$ para todo $i \neq j$ e seja $y^{\prime}$ o vetor tal que $\lambda_{i}^{\prime}$ é o $i$-ésimo elemento de $y^{\prime}$. Podemos verificar que $y^{\prime}$ pertence ao poliedro do programa linear (7.12), pois

$$
y^{\prime} A=y A-\lambda_{j}^{*} a_{j}=c-\lambda_{j}^{*} a_{j} .
$$

Como também sabemos que $x^{*}$ pertence ao poliedro de (7.11), podemos verificar pelo teorema da dualidade que $x^{*}$ é uma solução do programa (7.11) e $y^{\prime}$ é uma solução do programa (7.12), pois

$$
c^{\prime} x^{*}=\left(c-\lambda_{j}^{*} a_{j}\right) x^{*}=c x^{*}-\lambda_{j}^{*} a_{j} x^{*}=y^{*} b-\lambda_{j}^{*} \beta_{j}=y^{\prime} b .
$$

Mais precisamente, podemos verificar através desse argumento que toda solução de (7.9) também é uma solução de (7.11). Logo, se $\mathcal{F}^{\prime}$ é a face de $\mathcal{P}$ formada pelas soluções de (7.11), então todas as restrições de $A x \leq b$ que são ativas em $\mathcal{F}^{\prime}$ também são ativas em $\mathcal{F}$.

Por outro lado, podemos demonstrar que a restrição $a_{j} x \leq \beta_{j}$ não é ativa em $\mathcal{F}^{\prime}$. Suponha por contradição que essa restrição é ativa nessa face. Seja $y^{\prime \prime}$ uma solução de (7.12). Para todo $i$, vamos denotar por $\lambda_{i}^{\prime \prime}$ o $i$-ésimo elemento de $y^{\prime \prime}$. Podemos supor sem perda de generalidade que $\lambda_{j}^{\prime \prime}$ é máximo. Novamente pelo corolário 2.11 , temos que $\lambda_{j}^{\prime \prime}>0$.

Seja $\zeta_{j}^{*}:=\lambda_{j}^{\prime \prime}+\lambda_{j}^{*}$, seja $\zeta_{i}^{*}:=\lambda_{i}^{\prime \prime}$ para todo $i \neq j$ e seja $z^{*}$ o vetor tal que $\zeta_{i}^{*}$ é o $i$-ésimo elemento de $z^{*}$. Podemos verificar que $z^{*}$ é uma solução do programa linear (7.10), pois

- $z^{*} A=y^{\prime \prime} A+\lambda_{j}^{*} a_{j}=\left(y^{*} A-\lambda_{j}^{*} a_{j}\right)+\lambda_{j}^{*} a_{j}=y^{*} A$;

- $z^{*} b=y^{\prime \prime} b+\lambda_{j}^{*} \beta_{j}=\left(y^{*} b-\lambda_{j}^{*} \beta_{j}\right)+\lambda_{j}^{*} \beta_{j}=y^{*} b$.

Portanto, a existência de $z^{*}$ contradiz a maximalidade de $\lambda_{j}^{*}$, pois $\zeta_{j}^{*}=\lambda_{j}^{*}+\lambda_{j}^{\prime \prime}$ e $\lambda_{j}^{\prime \prime}>0$.

Podemos então concluir que a restrição $a_{j} x \leq \beta_{j}$ não é ativa em $\mathcal{F}^{\prime}$. Logo, existe $x^{\prime} \in \mathcal{F}^{\prime}$ tal que $a_{j} x^{\prime}<\beta_{j}$. Pelo corolário 2.20, sabemos que $x^{\prime}$ pertence ao fecho convexo de vetores que pertencem a faces minimais de $\mathcal{F}^{\prime}$, pois $\mathcal{P}$ é limitado. Portanto, existe um vetor $x^{\prime \prime}$ tal que $a_{j} x^{\prime \prime}<\beta_{j}$ e $x^{\prime \prime}$ pertence a uma face minimal $\mathcal{F}^{\prime \prime}$. Podemos verificar pelo corolário 2.21 que $x^{\prime \prime}$ é o único vetor de $\mathcal{F}^{\prime \prime}$, pois sabemos que $A x=0$ somente se $x=0$.

Como $\mathcal{P}$ é um poliedro inteiro, temos então que $x^{\prime \prime}$ é uma solução inteira de (7.11). Portanto,

$$
c x^{\prime \prime}-\lambda_{j}^{*} a_{j} x^{\prime \prime}=c^{\prime} x^{\prime \prime}=c^{\prime} x^{*}=c x^{*}-\lambda_{j}^{*} a_{j} x^{*}=c x^{*}-\lambda_{j}^{*} \beta_{j} .
$$

e podemos concluir que $c x^{*}-c x^{\prime \prime}=\lambda_{j}^{*}\left(\beta_{j}-a_{j} x^{\prime \prime}\right)$. Por outro lado, sabemos que $x^{\prime \prime}$ não é uma solução de (7.9), pois $a_{j} x \leq \beta_{j}$ é ativa em $\mathcal{F}$. Desse fato, segue que $c x^{\prime \prime}<c x^{*}$. Em particular, como $c, x^{\prime \prime}, x^{*}$ são vetores inteiros, temos $c x^{\prime \prime} \leq c x^{*}-1$. Por fim, como $a_{j} x^{\prime \prime} \geq \beta_{j}-1$ por hipótese,

$$
\lambda_{j}^{*}=\frac{c x^{*}-c x^{\prime \prime}}{\beta_{j}-a_{j} x^{\prime \prime}} \geq 1
$$

Através desse lema, podemos demonstrar que os sistemas geradores de Hilbert relativos às faces do poliedro são fortes. Esse fato nos permite obter naturalmente o limitante superior desejado.

Teorema 7.5. Seja $G=(V, E)$ um grafo e seja c um vetor que associa custos inteiros aos vértices desse grafo. Se $G$ é perfeito e existe pelo menos uma c-cobertura por cliques dos vértices de $G$, então existe uma c-cobertura por cliques $\mathcal{K}$ dos vértices de $G$ tal que a cardinalidade de $\mathcal{K}$ é mínima e $\mathcal{K}$ contém não mais do que $|V|$ tipos de clique. 
Demonstração. Se $G$ é um grafo perfeito, o sistema que define o poliedro do programa (7.7) é totalmente dual integral. Portanto, o limitante segue diretamente do lema 7.4 e do corolário 6.21.

Grötschel, LOVÁSZ E SCHRIJVER [1981] apresentaram um algoritmo polinomial para obter uma $c$-cobertura mínima de um grafo perfeito e demonstraram que a cobertura devolvida por esse algoritmo contém não mais do que $|V|^{2}+|V|$ tipos de clique. Embora a demonstração apresentada nesta seção resulte em um limitante melhor, não podemos obter diretamente um algoritmo polinomial a partir dela, pois a matriz sobre a qual definimos o sistema pode ser exponencialmente grande.

\subsection{Conjuntos gerados por circuitos}

Nesta e na próxima seção, vamos considerar conjuntos gerados por vetores de incidência de objetos combinatórios. Trata-se de uma maneira natural de estender para combinatória os resultados que foram demonstrados nos capítulos anteriores. Na verdade, pode-se dizer que alguns conjuntos desse tipo já foram vistos nas seções anteriores deste capítulo, pois as matrizes sobre as quais definimos sistemas totalmente dual integrais nessas seções são precisamente matrizes cujas linhas são vetores de incidência de objetos combinatórios, como $r$-cortes e cliques.

No entanto, os vetores que foram considerados nesses casos são definidos por restrições ativas na face de um poliedro. Os conjuntos sobre os quais trabalhamos nas seções anteriores, portanto, estavam sujeitos a restrições de otimalidade. Esse tipo de restrição não será mais considerado: vamos apresentar demonstrações que não utilizam diretamente conceitos de programação inteira e total dual integralidade. $\mathrm{O}$ foco desta seção em particular estará sobre circuitos de grafos.

Seja $G=(V, E)$ um grafo, sejam $C_{1}, \ldots, C_{m}$ os circuitos desse grafo e sejam $c_{1}, \ldots, c_{m}$ seus respectivos vetores de incidência. Como qualquer outro vetor indexado por arestas, esses vetores podem ser considerados funções que atribuem custos às arestas do grafo. A figura 7.6 mostra um exemplo de circuito e os custos atribuídos pelo respectivo vetor de incidência desse circuito.
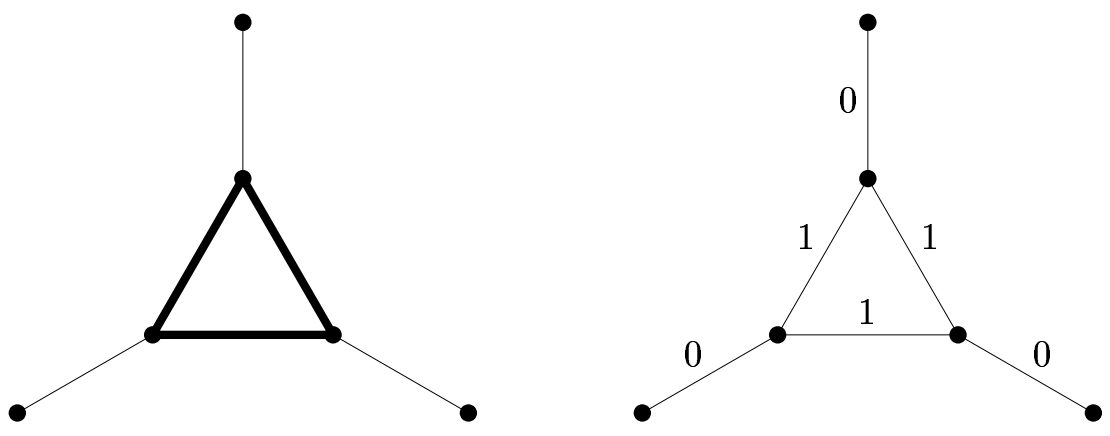

Figura 7.6: À esquerda, um exemplo de grafo e um de seus circuitos, identificado pelas arestas mais acentuadas. À direita, o respectivo vetor de incidência do circuito, representado por uma função de custos.

Se um vetor $c$ é gerado por uma combinação linear de $c_{1}, \ldots, c_{m}$, dizemos simplesmente que ele é uma função de custos gerada por uma combinação linear dos circuitos de $G$. A figura 7.7 mostra um exemplo de função gerada por combinação de circuitos.

A partir dessa extensão do conceito de combinação linear, podemos obter naturalmente definições de conjuntos gerados por circuitos, como por exemplo fecho convexo, fecho afim, fecho linear, cone, reticulado e cone inteiro. No entanto, salvo pelo fato de que os vetores considerados são 


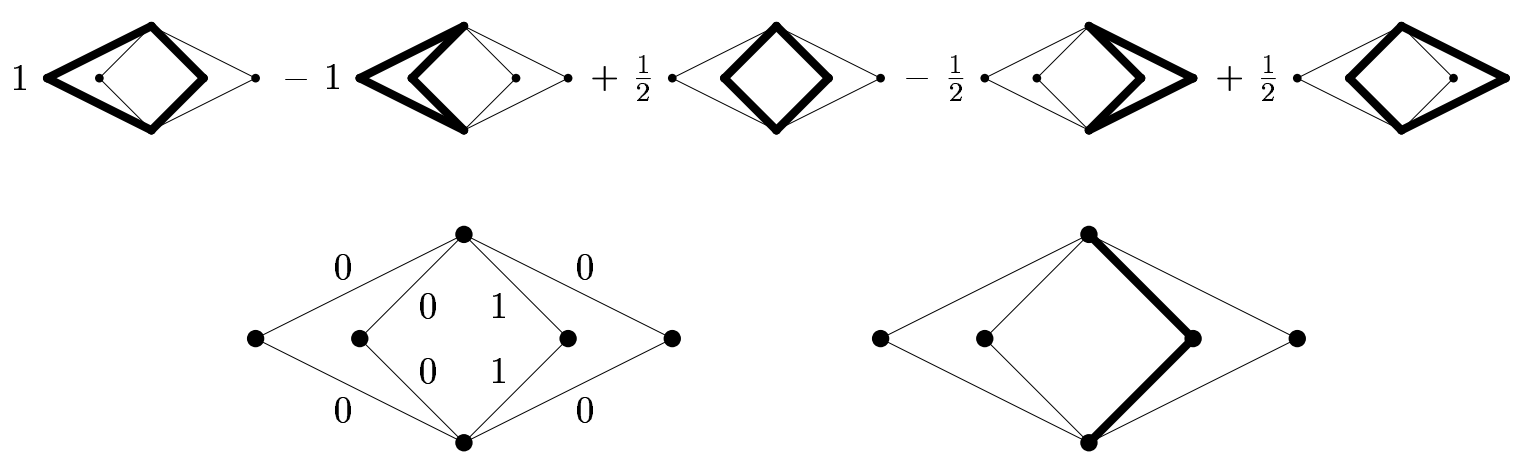

Figura 7.7: Uma combinação linear de circuitos e a função de custos gerada por essa combinação. Neste caso em particular, a função gerada é o vetor de incidência de um conjunto de arestas.

representações de circuitos, essas definições são puramente algébricas. Visto que estamos interessados em relacionar combinatória a álgebra linear, podemos considerar então a seguinte pergunta:

assim como $c_{1}, \ldots, c_{m}$ são representações vetoriais de circuitos, existem caracterizações em termos de teoria dos grafos para certos conjuntos gerados por esses vetores?

Mais precisamente, gostaríamos de caracterizar conjuntos gerados por circuitos sem a necessidade de utilizar diretamente o conceito de combinação linear. Para melhor esclarecer essa afirmação, vamos considerar a seguir uma caracterização para um conjunto gerado específico, o fecho linear.

\section{Fecho linear dos circuitos}

Como mencionado anteriormente, a definição de fecho linear para circuitos segue naturalmente da extensão do conceito de combinação linear. Formalmente, tal fecho é o conjunto

$$
\operatorname{lin}\left(c_{1}, \ldots, c_{m}\right)=\left\{c: c=\lambda_{1} c_{1}+\cdots+\lambda_{m} c_{m}, \text { onde } \lambda_{1}, \ldots, \lambda_{m} \in \mathbb{R}\right\} .
$$

Estamos interessados em obter condições necessárias e suficientes para que um dado vetor $c$ pertença a $\operatorname{lin}\left(c_{1}, \ldots, c_{m}\right)$. Como esse conjunto contém $c_{1}, \ldots, c_{m}$ e todos os vetores gerados por combinações lineares desses vetores de incidência, sabemos que tais condições precisam ser

(i) válidas para $c_{1}, \ldots, c_{m}$;

(ii) fechadas sob combinação linear.

Primeiramente, vamos considerar as arestas que não pertencem a circuitos. Dado um índice $i$, sabemos pela definição de vetor de incidência que uma aresta $e$ satisfaz $c_{i}(e)=0$ se e somente se $e$ não pertence a $C_{i}$. Logo, se $e$ não pertence a nenhum circuito do grafo, então $c_{i}(e)=0$ para todo índice $i$. Temos, portanto, uma propriedade que satisfaz (i). Ademais, como qualquer combinação linear de zeros gera zero, podemos verificar que tal propriedade também satisfaz (ii). Segue desse fato uma primeira condição necessária para que um vetor $c$ pertença ao fecho linear dos circuitos:

se e não pertence a nenhum circuito, então $c(e)=0$.

Embora essa condição seja bastante simples, podemos enunciá-la de forma ainda mais sucinta, pois sabemos que uma aresta não pertence a nenhum circuito se e somente se ela é uma ponte: 
se e é uma ponte, então $c(e)=0$.

Vamos considerar agora as arestas que pertencem a circuitos. Sejam $e, f$ arestas de $G$ e suponha que existe $i$ tal que $C_{i}$ contém $e, f$. Pela definição de vetor de incidência, sabemos que $c_{i}(e)=c_{i}(f)$. Suponha agora que existe um outro índice $j$ tal que $C_{j}$ também contém $e, f$. Nesse caso, sabemos analogamente que $c_{j}(e)=c_{j}(f)$. Ademais, podemos verificar a partir das duas igualdades que

$$
\lambda_{i} c_{i}(e)+\lambda_{j} c_{j}(e)=\lambda_{i} c_{i}(f)+\lambda_{j} c_{j}(f) \text { para quaisquer } \lambda_{i}, \lambda_{j} \in \mathbb{R} \text {. }
$$

A igualdade entre os custos dessas duas arestas, portanto, é uma propriedade válida para $c_{i}, c_{j}$ e fechada sob combinação linear desses dois vetores. Essa propriedade pode ser generalizada para quaisquer circuitos através do conceito de coparalelismo.

Sejam $e, f$ arestas de $G$ tais que cada uma delas pertence a pelo menos um circuito. Dizemos que essas arestas são coparalelas se todo circuito que contém uma delas também contém a outra. Podemos verificar que, se $e, f$ são coparalelas, então $c_{i}(e)=c_{i}(f)$ para todo $i$. Nesse caso, portanto, a igualdade (7.13) pode ser estendida naturalmente para quaisquer combinações de $c_{1}, \ldots, c_{m}$. Segue desse fato outra condição necessária para que um vetor $c$ pertença ao fecho linear dos circuitos:

se e, $f$ são arestas coparalelas, então $c(e)=c(f)$.

Vamos demonstrar a seguir que, quando consideradas simultaneamente, essas duas condições necessárias são também suficientes para que um vetor pertença ao conjunto $\operatorname{lin}\left(c_{1}, \ldots, c_{m}\right)$.

Primeiramente, cabe observar que o conceito de coparalelismo define uma relação simétrica, reflexiva e transitiva sobre o conjunto das arestas que pertencem a pelo menos um circuito. Portanto, tal conjunto pode ser particionado de acordo com as classes de equivalência definidas por essa relação. Ademais, tais classes são conjuntos maximais de arestas mutuamente coparalelas.

Sejam $B_{1}, \ldots, B_{l} \subseteq E$ esses subconjuntos maximais e seja $B \subseteq E$ o conjunto das pontes do grafo. Como uma aresta pertence a pelo menos um circuito se e somente se ela não é uma ponte, podemos verificar que os conjuntos $B, B_{1}, \ldots, B_{l}$ formam uma partição de $E$. Vamos denotar por $b, b_{1}, \ldots, b_{l}$ os respectivos vetores de incidência desses subconjuntos de arestas.

Suponha então que um vetor $c$, indexado pelas arestas de $G$, satisfaz as duas condições: $c(e)=0$ se $e$ é uma ponte e $c(e)=c(f)$ se $e, f$ são coparalelas. Pela definição de $B, B_{1}, \ldots, B_{l}$, sabemos que

- $c(e)=0$ se $e \in B$;

- para todo $k$, existe $\lambda_{k}$ tal que $c(e)=\lambda_{k}$ se $e \in B_{k}$.

Logo, como $\left\{B, B_{1}, \ldots, B_{l}\right\}$ é uma partição do conjunto de arestas do grafo, podemos verificar que:

$$
c=0 b+\lambda_{1} b_{1}+\cdots+\lambda_{l} b_{l} .
$$

Portanto, todo vetor que satisfaz as condições é gerado por uma combinação linear de $b_{1}, \ldots, b_{l}$. Como fechos lineares são fechados sob combinação linear, podemos concluir que as condições são suficientes se demonstrarmos que esses vetores pertencem ao fecho linear dos circuitos.

Primeiramente, vamos considerar um caso simples. Suponha que $G$ é o grafo da figura 7.8. O conjunto de arestas desse grafo é a união de dois circuitos distintos $C^{\prime}, C^{\prime \prime}$ e a intersecção desses circuitos contém somente uma aresta $e$. Podemos verificar que $\{e\}$ é um conjunto maximal de arestas mutuamente coparalelas. Vamos demonstrar que tal conjunto pertence ao fecho linear dos circuitos a partir do fato de que ele é a intersecção de $C^{\prime}, C^{\prime \prime}$. 


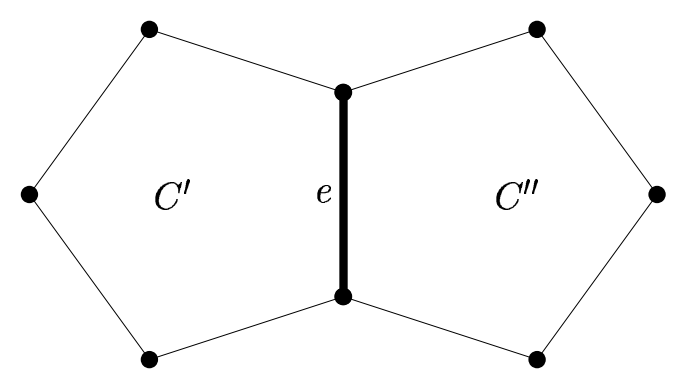

Figura 7.8: grafo obtido a partir da união de dois circuitos.

Sabemos que $\left(C^{\prime} \cup C^{\prime \prime}\right) \backslash\left(C^{\prime} \cap C^{\prime \prime}\right)$ é uma união de circuitos disjuntos. Seja $u$ o vetor de incidência dessa união, sejam $c^{\prime}, c^{\prime \prime}$ os respectivos vetores de incidência de $C^{\prime}, C^{\prime \prime}$ e seja $b_{k}$ o vetor de incidência de $\{e\}$. Como $C^{\prime} \cap C^{\prime \prime}=\{e\}$, podemos concluir que $c^{\prime}+c^{\prime \prime}-2 b_{k}=u$. Desse fato segue que

$$
2 b_{k}=c^{\prime}+c^{\prime \prime}-u \text {. }
$$

Como $u$ é o vetor de incidência de uma união de circuitos disjuntos, ele é gerado pela soma dos vetores de incidência desses circuitos. Logo, a partir da igualdade (7.15), podemos verificar que $b_{k}$ é gerado por uma combinação linear de circuitos.

Portanto, demonstramos que o vetor de incidência do conjunto $\{e\}$ pertence ao fecho linear dos circuitos. Mais precisamente, utilizamos um argumento que pode ser aplicado a qualquer conjunto que seja intersecção de dois circuitos. A partir desse fato, podemos generalizar essa demonstração para todos os conjuntos maximais de arestas mutuamente coparalelas através do lema a seguir.

Lema 7.6. Seja $G$ um grafo e seja $B_{k}$ um conjunto de arestas desse grafo. Se $B_{k}$ é um conjunto maximal de arestas mutuamente coparalelas em $G$, então $B_{k}$ é a intersecção de dois circuitos de $G$.

Demonstração. Seja $C$ um circuito de $G$ que contém $B_{k}$ e seja $\left\langle e_{0}, \ldots, e_{\left|B_{k}-1\right|}\right\rangle$ uma ordem cíclica de $B_{k}$ nesse circuito. Sabemos que existem caminhos $P_{0}, \ldots, P_{\left|B_{k}-1\right|}$ tais que $E\left(P_{0}\right), \ldots, E\left(P_{\left|B_{k}-1\right|}\right)$ formam uma partição de $C \backslash B_{k}$ e, para todo $i$, uma das pontas de $P_{i}$ pertence a $e_{i}$ e a outra pertence a $e_{(i+1) \bmod \left|B_{k}\right|}$. A figura 7.9 mostra um exemplo dessa partição de $C \backslash B_{k}$ em caminhos.

Sejam $\left(V_{0}, E_{0}\right), \ldots,\left(V_{\left|B_{k}-1\right|}, E_{\left|B_{k}-1\right|}\right)$ as componentes de $\left(V, E \backslash B_{k}\right)$ tais que, para todo $i$, as arestas de $P_{i}$ pertencem a $E_{i}$. Vamos demonstrar que essas componentes são mutuamente distintas. Podemos verificar que, se $i \neq j$ e existe um caminho em $\left(V, E \backslash B_{k}\right)$ que conecta $P_{i}$ a $P_{j}$, então existe um circuito que contém um subconjunto próprio de $B_{k}$. Como a existência de tal circuito contradiz a definição de coparalelismo, podemos concluir que $\left(V_{i}, E_{i}\right) \neq\left(V_{j}, E_{j}\right)$ se $i \neq j$.

Vamos demonstrar para todo $i$ que, se $u, v$ são as pontas do caminho $P_{i}$, então existem dois $(u, v)$-caminhos $P_{i}^{\prime}, P_{i}^{\prime \prime}$ no grafo $\left(V_{i}, E_{i}\right)$ tais que $E\left(P_{i}^{\prime}\right) \cap E\left(P_{i}^{\prime \prime}\right)=\emptyset$. Se o comprimento de $P_{i}$ é zero, podemos verificar que $P_{i}^{\prime}$ e $P_{i}^{\prime \prime}$ são aresta-disjuntos se $P_{i}^{\prime}=P_{i}$ e $P_{i}^{\prime \prime}=P_{i}$. Suponha então que o comprimento de $P_{i}$ é positivo. Nesse caso, existe um subconjunto não-vazio $E^{\prime}$ de $E_{i}$ tal que não existem $(u, v)$-caminhos em $\left(V_{i}, E_{i} \backslash E^{\prime}\right)$. Se $E^{\prime}$ contém uma única aresta, então tal aresta pertence a todos os circuitos que contém $B_{k}$. Como esse fato contradiz a maximalidade de $B_{k}$, temos que $E^{\prime}$ contém pelo menos duas arestas. Portanto, pelo teorema de Menger, podemos concluir que existem $(u, v)$-caminhos $P_{i}^{\prime}, P_{i}^{\prime \prime}$ tais que $E\left(P_{i}^{\prime}\right) \cap E\left(P_{i}^{\prime \prime}\right)=\emptyset$.

Como as componentes consideradas são mutuamente distintas, os conjuntos $E_{0}, \ldots, E_{\left|B_{k}-1\right|}$ são mutuamente disjuntos. Logo, podemos verificar que $E\left(P_{i}^{\prime}\right) \cap E\left(P_{j}^{\prime \prime}\right)=\emptyset$ para quaisquer $i, j$. Portanto, $B_{k} \cup E\left(P_{0}^{\prime}\right) \cup \ldots \cup E\left(P_{\left|B_{k}-1\right|}^{\prime}\right)$ e $B_{k} \cup E\left(P_{0}^{\prime \prime}\right) \cup \ldots \cup E\left(P_{\left|B_{k}-1\right|}^{\prime \prime}\right)$ são circuitos cuja intersecção é $B_{k}$. 


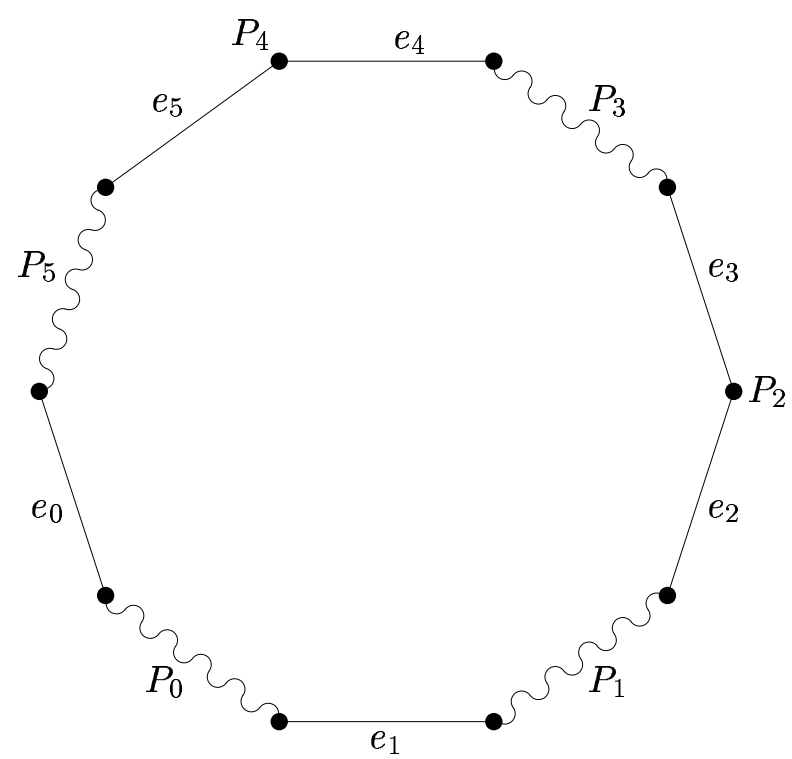

Figura 7.9: Um exemplo da partição descrita na demonstração do lema 7.6. Neste caso em particular, o comprimento de dois caminhos da partição é zero.

Recapitulando os argumentos desta subseção, podemos caracterizar o fecho linear dos circuitos através de uma propriedade que pode ser enunciada sem a necessidade do conceito de combinação linear. Obtemos, dessa maneira, a caracterização combinatória na qual estávamos interessados.

Teorema 7.7. Seja $G$ um grafo e seja $c$ um vetor indexado pelas arestas desse grafo. $O$ vetor $c$ pertence ao fecho linear dos circuitos de $G$ se e somente se $c(e)=0$ para toda ponte e e $c(e)=c(f)$ para quaisquer arestas coparalelas $e, f$.

Demonstração. A necessidade das condições segue do fato de que elas são válidas para o vetor de incidência de qualquer circuito de $G$ e são fechadas sob combinação linear. Suponha então que um vetor $c$ satisfaz as condições. Sabemos pela igualdade (7.14) que esse vetor é combinação linear de vetores de incidência de conjuntos maximais de arestas mutuamente coparalelas. Pelo lema 7.6, sabemos também que cada um desses conjuntos é a intersecção de dois circuitos. Portanto, pela igualdade (7.15), podemos concluir que o vetor $c$ pertence ao fecho linear dos circuitos do grafo.

Essa caracterização foi apresentada em um artigo de SEymour [1979]. No mesmo artigo, o autor apresenta descrições combinatórias para outros conjuntos gerados por circuitos. Um desses conjuntos é o reticulado, que pode ser caracterizado por propriedades similares às do fecho linear.

\section{Reticulado dos circuitos}

Estamos agora interessados em obter condições necessárias e suficientes para que um dado vetor pertença a ret $\left(c_{1}, \ldots, c_{m}\right)$. De maneira análoga ao fecho linear, podemos verificar que tais condições precisam ser válidas para $c_{1}, \ldots, c_{m}$ e fechadas sob combinação inteira.

Como qualquer combinação inteira de vetores inteiros gera um vetor inteiro, sabemos que todo vetor que pertence ao reticulado dos circuitos é inteiro. Apesar de simples, essa condição necessária permite o uso de argumentos que não poderiam ser aplicados sobre o fecho linear. Podemos, por exemplo, considerar propriedades relacionadas a paridade. 
Sabemos que a cardinalidade da intersecção entre um corte e qualquer circuito é par. A partir da definição de vetor de incidência, podemos formular essa propriedade da seguinte maneira:

se $V^{\prime} \subseteq V$, então $c_{i}\left(\delta\left(V^{\prime}\right)\right)$ é par para todo $i$.

Como qualquer soma e qualquer subtração de números pares gera um número par, podemos verificar que essa paridade é fechada sob combinação inteira. Logo, podemos concluir que um vetor $c$ pertence ao reticulado dos circuitos somente se ele é um vetor inteiro que atribui custo par aos cortes:

se $V^{\prime} \subseteq V$, então $c\left(\delta\left(V^{\prime}\right)\right)$ é par.

Vamos demonstrar que, se um vetor inteiro $c$ pertence a $\operatorname{lin}\left(c_{1}, \ldots, c_{m}\right)$ e atribui custo par aos cortes, então esse vetor pertence a $\operatorname{ret}\left(c_{1}, \ldots, c_{m}\right)$. Como essas hipóteses afirmam que $c$ pertence ao fecho linear dos circuitos, podemos considerar argumentos anteriores.

Em particular, sabemos a partir da igualdade (7.14) que $c$ é gerado por uma combinação linear

$$
\lambda_{1} b_{1}+\cdots+\lambda_{l} b_{l}
$$

onde os vetores $b_{1}, \ldots, b_{l}$ são vetores de incidência de subconjuntos maximais de arestas mutuamente coparalelas. Como esses subconjuntos são mutuamente disjuntos e $c$ é um vetor inteiro, podemos verificar que $\lambda_{1}, \ldots, \lambda_{k}$ são números inteiros. A combinação (7.16), portanto, é inteira.

Vamos considerar agora o lema 7.6. Para todo $k$, podemos verificar a partir desse lema e da igualdade (7.15) que o vetor $2 b_{k}$ é uma combinação inteira de $c^{\prime}, c^{\prime \prime}, u$, onde $c^{\prime}, c^{\prime \prime}$ são vetores de incidência de circuitos e $u$ é gerado por uma soma de vetores de incidência de circuitos. Segue, portanto, que os vetores $2 b_{1}, \ldots, 2 b_{l}$ pertencem ao reticulado dos circuitos.

Vetores que pertencem ao reticulado dos circuitos são particularmente interessantes devido ao fato de que reticulados são fechados sob combinação inteira. Sejam $\mu_{1}, \ldots, \mu_{l}$ inteiros e seja

$$
d:=c+\mu_{1} 2 b_{1}+\cdots+\mu_{l} 2 b_{l} .
$$

Se demonstrarmos que $c$ pertence a ret $\left(c_{1}, \ldots, c_{m}\right)$, podemos verificar que $d$ também pertence a $\operatorname{ret}\left(c_{1}, \ldots, c_{m}\right)$. Analogamente, se demonstrarmos que $d$ pertence ao reticulado dos circuitos, podemos concluir que $c$ também pertence a esse reticulado, pois pela definição de $d$ temos que

$$
c=d-\mu_{1} 2 b_{1}-\cdots-\mu_{l} 2 b_{l} .
$$

Podemos então considerar uma estratégia de redução: ao invés de demonstrar que $c$ pertence a ret $\left(c_{1}, \ldots, c_{m}\right)$, vamos demonstrar que existem inteiros $\mu_{1}, \ldots, \mu_{l}$ tais que $d$ pertence ao reticulado dos circuitos. Como $c$ é gerado pela combinação (7.16), sabemos a partir da definição (7.17) que

$$
d=\left(\lambda_{1}+\mu_{1} 2\right) b_{1}+\cdots+\left(\lambda_{l}+\mu_{l} 2\right) b_{l} .
$$

Dentre os valores inteiros que podem ser atribuídos aos coeficientes, são escolhas naturais aqueles que simplificam o vetor $d$. Vamos considerar, por exemplo, que $\mu_{1}, \ldots, \mu_{l}$ são os inteiros tais que

$$
\lambda_{k}+\mu_{k} 2=\lambda_{k} \bmod 2 \text { para todo } k \text {. }
$$

Nesse caso, podemos verificar que $\left(\lambda_{1}+\mu_{1} 2\right), \ldots,\left(\lambda_{l}+\mu_{l} 2\right)$ pertencem ao conjunto $\{0,1\}$. Portanto, como $b_{1}, \ldots, b_{l}$ são vetores de incidência de conjuntos mutuamente disjuntos, podemos concluir que qualquer elemento de $d$ é igual a 0 ou 1 para essa escolha específica de coeficientes. 
Quando uma função atribui custo zero a algumas arestas, podemos em alguns casos desconsiderar essas arestas através dos conceitos de restrição e extensão. Seja $\left(V, E^{\prime}\right)$ um subgrafo de $G$ tal que

$$
E^{\prime}=\{e \in E: d(e)>0\} .
$$

A restrição de $d$ ao conjunto $E^{\prime}$ é um vetor $d^{\prime}$, indexado pelas arestas de $\left(V, E^{\prime}\right)$, tal que $d^{\prime}(e)=d(e)$ para todo $e \in E^{\prime}$. A extensão de $d^{\prime}$ ao conjunto $E$ é um vetor $d^{+}$, indexado pelas arestas de $G$, tal que $d^{+}(e)=d^{\prime}(e)$ se $e$ pertence a $E^{\prime}$ e $d^{+}(e)=0$ caso contrário. A partir dessa definição, sabemos que $d^{+}=d$. A figura 7.10 mostra um exemplo de restrição e extensão.
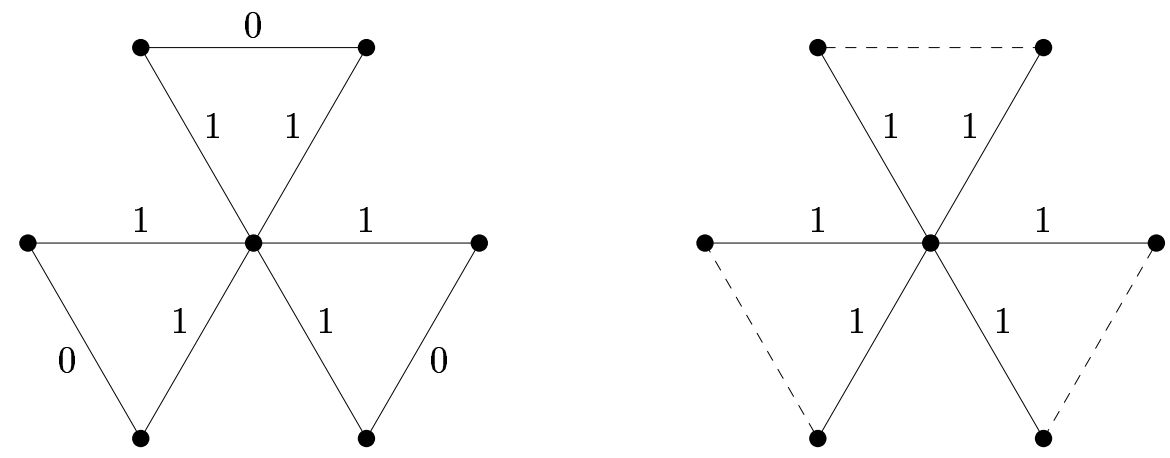

Figura 7.10: À esquerda, uma função de custos definida sobre um grafo. À direita, a restrição dessa função ao conjunto das arestas cujo custo é positivo. A extensão dessa restrição ao conjunto original é a função original.

Uma restrição pode ser utilizada para considerarmos somente as arestas do conjunto $E^{\prime}$ quando a propriedade que estamos interessados em demonstrar é fechada sob extensão. No presente contexto, estamos interessados em demonstrar que $d$ pertence ao reticulado dos circuitos de $G$. Podemos verificar que a extensão do vetor de incidência de um circuito do subgrafo $\left(V, E^{\prime}\right)$ ao conjunto $E$ é o vetor de incidência de um circuito do grafo $G$. Portanto, podemos concluir que $d$ pertence a ret $\left(c_{1}, \ldots, c_{m}\right)$ se demonstrarmos que $d^{\prime}$ pertence ao reticulado dos circuitos de $\left(V, E^{\prime}\right)$.

Vamos demonstrar tal pertinência a partir da hipótese de que $c$ atribui custo par aos cortes. Podemos verificar que os elementos de $2 b_{k}$ são iguais a 0 ou 2 para todo $k$. Logo, $2 b_{1}, \ldots, 2 b_{l}$ são vetores que atribuem custo par aos cortes de $G$. Portanto, sabemos pela definição (7.17) que o vetor $d$ também atribui custo par aos cortes desse grafo.

Dado um conjunto de vértices $V^{\prime}$, vamos denotar por $\delta\left(V^{\prime}\right)$ o corte desse subconjunto em relação ao grafo $G$ e por $\delta^{\prime}\left(V^{\prime}\right)$ o corte do subconjunto em relação ao subgrafo $\left(V, E^{\prime}\right)$. Pela definição de restrição, sabemos que $d^{\prime}\left(\delta^{\prime}\left(V^{\prime}\right)\right)$ é igual a $d\left(\delta\left(V^{\prime}\right)\right)$ para todo $V^{\prime} \subseteq V$. A partir desse fato, podemos concluir que o vetor $d^{\prime}$ atribui custo par aos cortes de $\left(V, E^{\prime}\right)$.

Como estamos supondo que os elementos de $d$ são iguais a 0 ou 1 , sabemos que $d^{\prime}$ atribui custo 1 a todas as arestas em $E^{\prime}$. Logo, a demonstração pode ser concluída a partir do lema a seguir.

Lema 7.8. Seja $\left(V, E^{\prime}\right)$ um grafo e seja $d^{\prime}$ um vetor indexado pelas arestas desse grafo. Se $d^{\prime}(e)=1$ para todo e e $d^{\prime}$ atribui custo par aos cortes, então $d^{\prime}$ pertence ao reticulado dos circuitos de $\left(V, E^{\prime}\right)$.

Demonstração. Como $d^{\prime}(e)=1$ para todo $e$ e $d^{\prime}$ atribui custo par aos cortes de $\left(V, E^{\prime}\right)$, podemos verificar que o grau de cada vértice desse grafo é par. Logo, sabemos que $E^{\prime}$ é uma união de circuitos disjuntos do grafo $\left(V, E^{\prime}\right)$. Portanto, a partir da definição de vetor de incidência, podemos concluir que o dado vetor $d^{\prime}$ é gerado pela soma dos vetores de incidência desses circuitos disjuntos. 
Recapitulando os argumentos da subseção, podemos enfim caracterizar o reticulado dos circuitos.

Teorema 7.9. Seja $G$ um grafo e seja c um vetor indexado pelas arestas desse grafo. O vetor $c$ pertence ao reticulado dos circuitos de $G$ se e somente se $c$ é inteiro, c atribui custo par aos cortes de $G, c(e)=0$ para toda ponte e e $c(e)=c(f)$ para quaisquer arestas coparalelas $e, f$.

Demonstração. A necessidade das condições segue do fato de que elas são válidas para o vetor de incidência de qualquer circuito de $G$ e são fechadas sob combinação inteira. Suponha então que um vetor $c$ satisfaz as condições. Sabemos que, para quaisquer inteiros $\mu_{1}, \ldots, \mu_{l}$, o vetor $c$ pertence ao reticulado dos circuitos se o vetor $d$ descrito pela definição (7.17) pertence ao reticulado dos circuitos. Em particular, existem $\mu_{1}, \ldots, \mu_{l}$ tais que os elementos de $d$ são iguais a 0 ou 1 . Precisamos somente demonstrar que a restrição desse vetor $d$ ao conjunto de arestas às quais $d$ atribui custo 1 pertence ao reticulado dos circuitos do subgrafo correspondente. Esse fato segue diretamente do lema 7.8.

A atribuição de custo par aos cortes, portanto, é a propriedade que indica se um vetor inteiro que pertence ao fecho linear dos circuitos pertence ou não ao reticulado dos circuitos também.

\section{Cone dos circuitos}

Além de exibir caracterizações para o fecho linear e o reticulado dos circuitos, SEYmour [1979] também demonstrou a necessidade e a suficiência de condições para que um vetor pertença ao cone gerado pelos circuitos. Essa demonstração, apesar de consideravelmente mais técnica do que as demonstrações apresentadas para o fecho linear e o reticulado, baseia-se em argumentos similares. Em particular, ela também utiliza uma estratégia de redução.

Primeiramente, podemos verificar que quaisquer vetores que pertencem ao cone dos circuitos são não-negativos, pois esses vetores são gerados por combinações não-negativas de vetores de incidência de circuitos. Um vetor que pertence ao cone, no entanto, não é necessariamente inteiro. Esse fato impossibilita o uso de quaisquer argumentos baseados na paridade dos custos.

Não é necessário, porém, desconsiderar o fato de que a cardinalidade da intersecção entre um corte e um circuito é par. A partir desse fato, podemos demonstrar propriedades que podem ser enunciadas sem a necessidade do conceito de paridade. Sabemos, por exemplo, que um conjunto não-vazio de arestas cuja cardinalidade é par contém pelo menos duas arestas. Logo, temos que

para todo indice $i$, todo $V^{\prime} \subseteq V$ e todo e $\in \delta\left(V^{\prime}\right)$, temos $\left|C_{i} \cap\left(\delta\left(V^{\prime}\right) \backslash\{e\}\right)\right| \geq 1$ se e $\in C_{i}$.

Pela definição de vetor de incidência, podemos reformular essa propriedade da seguinte maneira:

para todo índice $i$, todo $V^{\prime} \subseteq V$ e todo e $\in \delta\left(V^{\prime}\right)$, temos $c_{i}\left(\delta\left(V^{\prime}\right) \backslash\{e\}\right) \geq 1$ se $c_{i}(e)=1$.

Como vetores de incidência são não-negativos, podemos também verificar que, para todo índice $i$, todo $V^{\prime} \subseteq V$ e todo $e \in \delta\left(V^{\prime}\right)$, temos $c_{i}\left(\delta\left(V^{\prime}\right) \backslash\{e\}\right) \geq 0$. Portanto, como $c_{i}(e)$ é igual a 0 ou 1 ,

para todo índice $i$, todo $V^{\prime} \subseteq V$ e todo $e \in \delta\left(V^{\prime}\right)$, temos $c_{i}\left(\delta\left(V^{\prime}\right) \backslash\{e\}\right) \geq c_{i}(e)$.

Podemos verificar que essa propriedade é fechada sob combinação não-negativa. Logo, seguem desses argumentos duas condições necessárias para que um vetor $c$ pertença ao cone dos circuitos:

(i) $c(e) \leq c\left(\delta\left(V^{\prime}\right) \backslash\{e\}\right) \quad$ para todo $V^{\prime} \subseteq V$ e todo $e \in \delta\left(V^{\prime}\right)$;

(ii) $c(e) \geq 0 \quad$ para todo $e \in E$. 
Dizemos que um vetor é admissível se ele satisfaz essas duas condições. Vamos demonstrar que todo vetor admissível $c$ pertence ao cone dos circuitos.

Consideraremos uma estratégia de redução similar à que utilizamos para caracterizar o reticulado. Sabemos que o vetor $c_{i}$ pertence ao cone dos circuitos para todo $i$. Logo, se demonstrarmos que o vetor $d:=c-\lambda_{i} c_{i}$ pertence a cone $\left(c_{1}, \ldots, c_{m}\right)$ para algum $\lambda_{i} \geq 0$, podemos concluir que $c$ pertence ao cone dos circuitos, pois $c=d+\lambda_{i} c_{i}$. Escolhas particularmente interessantes para o coeficiente $\lambda_{i}$ são aquelas para as quais o vetor $d$ também satisfaz as condições (i) e (ii):

$$
\begin{array}{ll}
c(e)-\lambda_{i} c_{i}(e) \leq c\left(\delta\left(V^{\prime}\right) \backslash\{e\}\right)-\lambda_{i} c_{i}\left(\delta\left(V^{\prime}\right) \backslash\{e\}\right) & \text { para todo } V^{\prime} \subseteq V \text { e todo } e \in \delta\left(V^{\prime}\right) ; \\
c(e)-\lambda_{i} c_{i}(e) \geq 0 & \text { para todo } e \in E .
\end{array}
$$

Se nos restringirmos a esse tipo de coeficiente, temos naturalmente limitantes superiores para $\lambda_{i}$. Colocando $\lambda_{i}$ em evidência, podemos verificar que essas condições são satisfeitas se e somente se

$$
\lambda_{i} \leq \frac{c\left(\delta\left(V^{\prime}\right) \backslash\{e\}\right)-c(e)}{c_{i}\left(\delta\left(V^{\prime}\right) \backslash\{e\}\right)-c_{i}(e)} \quad \lambda_{i} \leq \frac{c(e)}{c_{i}(e)}
$$

para todo $V^{\prime} \subseteq V$ e todo $e \in \delta\left(V^{\prime}\right) \quad$ para todo $e \in E$ tal que $c_{i} \neq 0$.

tais que $c_{i}\left(\delta\left(V^{\prime}\right) \backslash\{e\}\right)-c_{i}(e) \neq 0$;

Pela definição de vetor de incidência, sabemos que para todo $i$ existe $e \in E$ tal que $c_{i}(e) \neq 0$. Logo, o segundo limitante nos permite definir, para todo $i$, um número não-negativo $\lambda_{c, i}^{*}$ tal que

$$
\lambda_{c, i}^{*}=\max \left\{\lambda_{i}: c-\lambda_{i} c_{i} \text { é admissível }\right\} .
$$

Ademais, podemos verificar a partir dessa definição de $\lambda_{c, i}^{*}$ que, se um vetor $d$ é igual a $c-\lambda_{c, i}^{*} c_{i}$ para algum índice $i$, então pelo menos uma dentre as duas afirmações a seguir é verdadeira:

- $d(e)=0$ para algum $e \in E$;

- $d(e)=d\left(\delta\left(V^{\prime}\right) \backslash\{e\}\right)$ para algum $V^{\prime} \subseteq V$ e algum $e \in \delta\left(V^{\prime}\right)$.

Se $d(e)=0$ para algum $e \in E$, podemos utilizar novamente os conceitos de restrição e extensão. Podemos verificar que, se $d$ é admissível, então a restrição desse vetor às arestas cujo custo atribuído por $d$ é não-nulo também é admissível. Podemos verificar também que, se a restrição pertence ao cone dos circuitos do grafo correspondente, $d$ pertence ao cone dos circuitos de $G$.

Logo, precisamos mostrar nesse caso que a restrição pertence ao cone dos circuitos. Sabemos que tal restrição é admissível e indexada pelas arestas de um grafo estritamente menor que $G$. Podemos então considerar uma demonstração por indução no tamanho do grafo. Mais precisamente, vamos demonstrar por indução em $|E|$ que, se um vetor é admissível, então ele pertence ao cone dos circuitos. A base da indução é o caso em que $|E|=0$.

Vamos então verificar como podemos obter um grafo menor no caso em que $d(e)=d\left(\delta\left(V^{\prime}\right) \backslash\{e\}\right)$ para algum $V^{\prime} \subseteq V$ e algum $e \in \delta\left(V^{\prime}\right)$. Utilizaremos o conceito de contração de vértices. Suponha que $V^{\prime} \subseteq V$ é não-vazio. O grafo $\left(V / V^{\prime}, E^{\prime}\right)$ é obtido a partir da contração de $V^{\prime}$ em $G$ se

$$
\begin{aligned}
V / V^{\prime} & =\left(V \backslash V^{\prime}\right) \cup\left\{v^{\prime}\right\}, \text { onde } v^{\prime} \notin V ; \\
E^{\prime} & =\left(\left(E \backslash\left\{e: e \subseteq V^{\prime}\right\}\right) \backslash\left\{e_{1}, \ldots, e_{l}\right\}\right) \cup\left\{e_{1}^{\prime}, \ldots, e_{l}^{\prime}\right\}, \text { onde } \\
\left\{e_{1}, \ldots, e_{l}\right\} & =\delta\left(V^{\prime}\right) \text { e } \\
e_{i}^{\prime} & =\left\{u, v^{\prime}\right\} \text { para todo } i, \text { onde } e_{i}=\{u, v\} \text { e } v \in V^{\prime} .
\end{aligned}
$$


Dizemos que $e_{1}^{\prime}, \ldots, e_{l}^{\prime}$ são as arestas correspondentes a $e_{1}, \ldots, e_{l}$ em $\left(V / V^{\prime}, E^{\prime}\right)$ e que $v^{\prime}$ é a identificação de $V^{\prime}$. Apesar dessa definição ser consideravelmente longa, ela pode ser interpretada de maneira bastante natural. Informalmente, obtemos esse grafo quando um conjunto de vértices é colapsado em um único vértice. A adaptação de $d$ relativa à contração de $V^{\prime}$ é um vetor $d^{\prime}$, indexado pelas arestas de $\left(V / V^{\prime}, E^{\prime}\right)$, tal que $d^{\prime}\left(e_{i}^{\prime}\right)=d\left(e_{i}\right)$ para todo $i$ e $d^{\prime}(e)=d(e)$ se $e \in E^{\prime} \backslash\left\{e_{1}^{\prime}, \ldots, e_{l}^{\prime}\right\}$. A expansão de $d^{\prime}$ relativa à contração de $V^{\prime}$ é um vetor $d^{+}$, indexado pelas arestas de $G$, tal que $d^{+}\left(e_{i}\right)=d^{\prime}\left(e_{i}^{\prime}\right)$ para todo $i, d^{+}(e)=d^{\prime}(e)$ se $e \in E^{\prime} \backslash\left\{e_{1}^{\prime}, \ldots, e_{l}^{\prime}\right\}$ e $d^{+}(e)=0$ se $e \subseteq V^{\prime}$. A figura 7.11 mostra um exemplo de contração, adaptação e expansão.
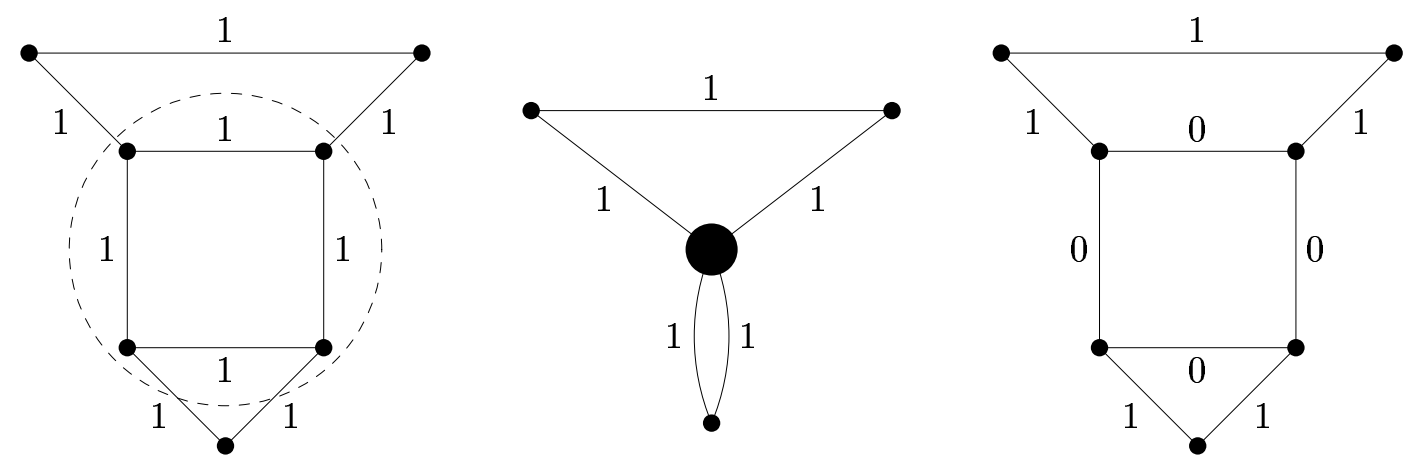

Figura 7.11: da esquerda para a direita, uma função de custos definida sobre um grafo, a adaptação dessa função relativa à contração do conjunto de vértices indicado pelo círculo e a expansão dessa adaptação relativa a essa contração.

Como estamos supondo que existe pelo menos uma aresta em $\delta\left(V^{\prime}\right)$, podemos verificar que o conjunto $V \backslash V^{\prime}$ é não-vazio. Logo, como $\delta\left(V^{\prime}\right)=\delta\left(V \backslash V^{\prime}\right)$, podemos considerar a contração desse conjunto complementar. Seja $V^{\prime \prime}:=V \backslash V^{\prime}$, seja $\left(V / V^{\prime \prime}, E^{\prime \prime}\right)$ o grafo obtido a partir da contração de $V^{\prime \prime}$ e seja $d^{\prime \prime}$ é a adaptação de $d$ a essa contração. Vamos supor que $e_{1}^{\prime \prime}, \ldots, e_{l}^{\prime \prime}$ são as arestas correspondentes a $e_{1}, \ldots, e_{l}$ em $\left(V / V^{\prime \prime}, E^{\prime \prime}\right)$. A figura 7.12 mostra essas duas contrações.

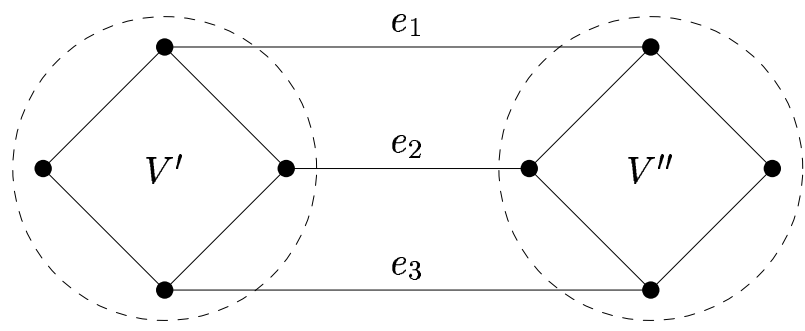

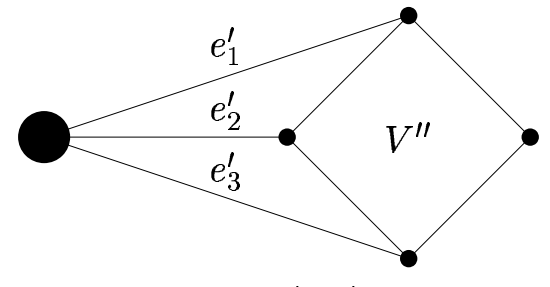

$\left(V / V^{\prime}, E^{\prime}\right)$

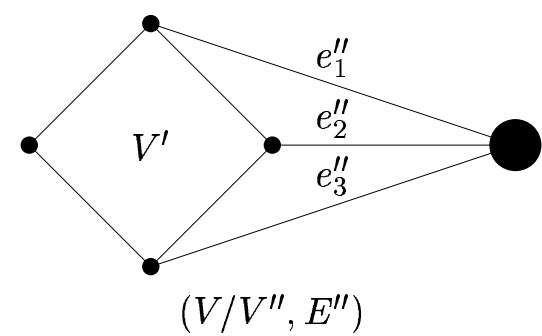

Figura 7.12: exemplo de um grafo obtido a partir da contração de um conjunto de vértices e de um grafo obtido a partir da contração do conjunto complementar. 
Vamos denotar por $\delta^{\prime}\left(V^{\prime \prime}\right)$ o corte de $V^{\prime \prime}$ em $\left(V / V^{\prime}, E^{\prime}\right)$ e por $\delta^{\prime \prime}\left(V^{\prime}\right)$ o corte de $V^{\prime}$ em $\left(V / V^{\prime \prime}, E^{\prime \prime}\right)$. Podemos verificar que, se $d$ é admissível para $G$, então $d^{\prime}$ é admissível para $\left(V / V^{\prime}, E^{\prime}\right)$ e $d^{\prime \prime}$ é admissível para $\left(V / V^{\prime \prime}, E^{\prime \prime}\right)$. Logo, se os dois grafos são menores que $G$, podemos aplicar a hipótese de indução e concluir que $d^{\prime}$ é gerado por uma combinação não-negativa dos circuitos de $\left(V / V^{\prime}, E^{\prime}\right)$ e $d^{\prime \prime}$ é gerado por uma combinação não-negativa dos circuitos de $\left(V / V^{\prime \prime}, E^{\prime \prime}\right)$. Mais formalmente,

- existem $\lambda_{1}^{\prime}, \ldots, \lambda_{m^{\prime}}^{\prime}>0$ tais que $d^{\prime}=\lambda_{1}^{\prime} c_{1}^{\prime}+\cdots+\lambda_{m^{\prime}}^{\prime} c_{m^{\prime}}^{\prime}$, onde $c_{1}^{\prime}, \ldots, c_{m^{\prime}}^{\prime}$ são os respectivos vetores de incidência de circuitos $C_{1}^{\prime}, \ldots, C_{m^{\prime}}^{\prime} \operatorname{de}\left(V / V^{\prime}, E^{\prime}\right)$;

- existem $\lambda_{1}^{\prime \prime}, \ldots, \lambda_{m^{\prime \prime}}^{\prime \prime}>0$ tais que $d^{\prime \prime}=\lambda_{1}^{\prime \prime} c_{1}^{\prime \prime}+\cdots+\lambda_{m^{\prime \prime}}^{\prime \prime} c_{m^{\prime \prime}}^{\prime \prime}$, onde $c_{1}^{\prime \prime}, \ldots, c_{m^{\prime \prime}}^{\prime \prime}$ são os respectivos vetores de incidência de circuitos $C_{1}^{\prime \prime}, \ldots, C_{m^{\prime \prime}}^{\prime \prime}$ de $\left(V / V^{\prime \prime}, E^{\prime \prime}\right)$.

Estamos interessados em obter, a partir dessas duas combinações, uma combinação não-negativa dos circuitos de $G$ que gera $d$. Primeiramente, podemos supor sem perda de generalidade que

- existe $r^{\prime} \leq m^{\prime}$ tal que $C_{i^{\prime}}^{\prime}$ intersecta $\delta^{\prime}\left(V^{\prime \prime}\right)$ se e somente se $i^{\prime}>r^{\prime}$;

- existe $r^{\prime \prime} \leq m^{\prime \prime}$ tal que $C_{i^{\prime \prime}}^{\prime \prime}$ intersecta $\delta^{\prime \prime}\left(V^{\prime}\right)$ se e somente se $i^{\prime \prime}>r^{\prime \prime}$.

Temos então que, se $r:=r^{\prime}+r^{\prime \prime}$ e $c_{1}^{+}, \ldots, c_{r}^{+}$são as expansões de $c_{1}^{\prime}, \ldots, c_{r^{\prime}}^{\prime}, c_{1}^{\prime \prime}, \ldots, c_{r^{\prime \prime}}^{\prime \prime}$ relativas às respectivas contrações, então $c_{1}^{+}, \ldots, c_{r}^{+}$são vetores de incidência de circuitos de $G$.

Por outro lado, a expansão de $c_{i^{\prime}}^{\prime}$ não é o vetor de incidência de um circuito de $G$ se $i^{\prime}>r^{\prime}$. Da mesma forma, a expansão de $c_{i^{\prime \prime}}^{\prime \prime}$ não é o vetor de incidência de um circuito de $G$ se $i^{\prime \prime}>r^{\prime \prime}$. No entanto, em alguns casos podemos obter um circuito de $G$ a partir de $C_{i^{\prime}}^{\prime}$ e $C_{i^{\prime \prime}}^{\prime \prime}$. Suponha, por exemplo, que existem $j, k$ tais que $C_{i^{\prime}}^{\prime} \cap \delta^{\prime}\left(V^{\prime \prime}\right)=\left\{e_{j}^{\prime}, e_{k}^{\prime}\right\}$ e $C_{i^{\prime \prime}}^{\prime \prime} \cap \delta^{\prime \prime}\left(V^{\prime}\right)=\left\{e_{j}^{\prime \prime}, e_{k}^{\prime \prime}\right\}$. Nesse caso,

$$
\left(C_{i^{\prime}}^{\prime} \backslash\left\{e_{j}^{\prime}, e_{k}^{\prime}\right\}\right) \cup\left(C_{i^{\prime \prime}}^{\prime \prime} \backslash\left\{e_{j}^{\prime \prime}, e_{k}^{\prime \prime}\right\}\right) \cup\left\{e_{j}, e_{k}\right\}
$$

é um circuito em $G$. A figura 7.13 mostra um exemplo simples dessa combinação de circuitos.

Vamos então demonstrar que os circuitos nos quais estamos interessados contém exatamente duas arestas que pertencem ao corte. Vamos primeiramente demonstrar esse fato para $\left(V / V^{\prime}, E^{\prime}\right)$ :

para todo $i^{\prime}$ tal que $C_{i^{\prime}}^{\prime} \cap \delta^{\prime}\left(V^{\prime \prime}\right) \neq \emptyset$, temos $\left|C_{i^{\prime}}^{\prime} \cap \delta^{\prime}\left(V^{\prime \prime}\right)\right|=2$.

Sabemos pela hipótese sobre $d$ que existe $j$ tal que $d\left(e_{j}\right)=d\left(\delta\left(V^{\prime}\right) \backslash\left\{e_{j}\right\}\right)$. Logo, temos que

$$
d^{\prime}\left(e_{j}^{\prime}\right)=d^{\prime}\left(\delta^{\prime}\left(V^{\prime \prime}\right) \backslash\left\{e_{j}^{\prime}\right\}\right)
$$

Podemos verificar que $d^{\prime}-\lambda_{i^{\prime}}^{\prime} c_{i^{\prime}}^{\prime}$ é um vetor admissível em $\left(V / V^{\prime}, E^{\prime}\right)$ para todo $i^{\prime}$. Segue que

$$
d^{\prime}\left(e_{j}^{\prime}\right)-\lambda_{i^{\prime}}^{\prime} c_{i^{\prime}}^{\prime}\left(e_{j}^{\prime}\right) \leq d^{\prime}\left(\delta^{\prime}\left(V^{\prime \prime}\right) \backslash\left\{e_{j}^{\prime}\right\}\right)-\lambda_{i^{\prime}}^{\prime} c_{i^{\prime}}^{\prime}\left(\delta^{\prime}\left(V^{\prime \prime}\right) \backslash\left\{e_{j}^{\prime}\right\}\right) .
$$

Suponha então que $C_{i^{\prime}}^{\prime} \cap \delta^{\prime}\left(V^{\prime \prime}\right) \neq \emptyset$ para algum $i^{\prime}$. Como $\left|C_{i^{\prime}}^{\prime} \cap \delta^{\prime}\left(V^{\prime \prime}\right)\right|$ é par, existe pelo menos um $k \neq j$ tal que $e_{k}^{\prime}$ pertence a essa intersecção. Logo, como $c_{i^{\prime}}^{\prime}\left(e_{k}^{\prime}\right)=1$, podemos verificar que

$$
\begin{aligned}
\lambda_{i^{\prime}}^{\prime} c_{i^{\prime}}^{\prime}\left(\delta^{\prime}\left(V^{\prime \prime}\right) \backslash\left\{e_{j}^{\prime}\right\}\right) & =\lambda_{i^{\prime}}^{\prime} c_{i^{\prime}}^{\prime}\left(\delta^{\prime}\left(V^{\prime \prime}\right) \backslash\left\{e_{j}^{\prime}, e_{k}^{\prime}\right\}\right)+\lambda_{i^{\prime}}^{\prime} c_{i^{\prime}}^{\prime}\left(e_{k}^{\prime}\right) \\
& =\lambda_{i^{\prime}}^{\prime} c_{i^{\prime}}^{\prime}\left(\delta^{\prime}\left(V^{\prime \prime}\right) \backslash\left\{e_{j}^{\prime}, e_{k}^{\prime}\right\}\right)+\lambda_{i^{\prime}}^{\prime} .
\end{aligned}
$$

Segue então que a diferença $d^{\prime}\left(\delta^{\prime}\left(V^{\prime \prime}\right) \backslash\left\{e_{j}^{\prime}\right\}\right)-\lambda_{i^{\prime}}^{\prime} c_{i^{\prime}}^{\prime}\left(\delta^{\prime}\left(V^{\prime \prime}\right) \backslash\left\{e_{j}^{\prime}\right\}\right)$ pode ser reformulada como

$$
d^{\prime}\left(\delta^{\prime}\left(V^{\prime \prime}\right) \backslash\left\{e_{j}^{\prime}\right\}\right)-\lambda_{i^{\prime}}^{\prime} c_{i^{\prime}}^{\prime}\left(\delta^{\prime}\left(V^{\prime \prime}\right) \backslash\left\{e_{j}^{\prime}, e_{k}^{\prime}\right\}\right)-\lambda_{i^{\prime}}^{\prime}
$$



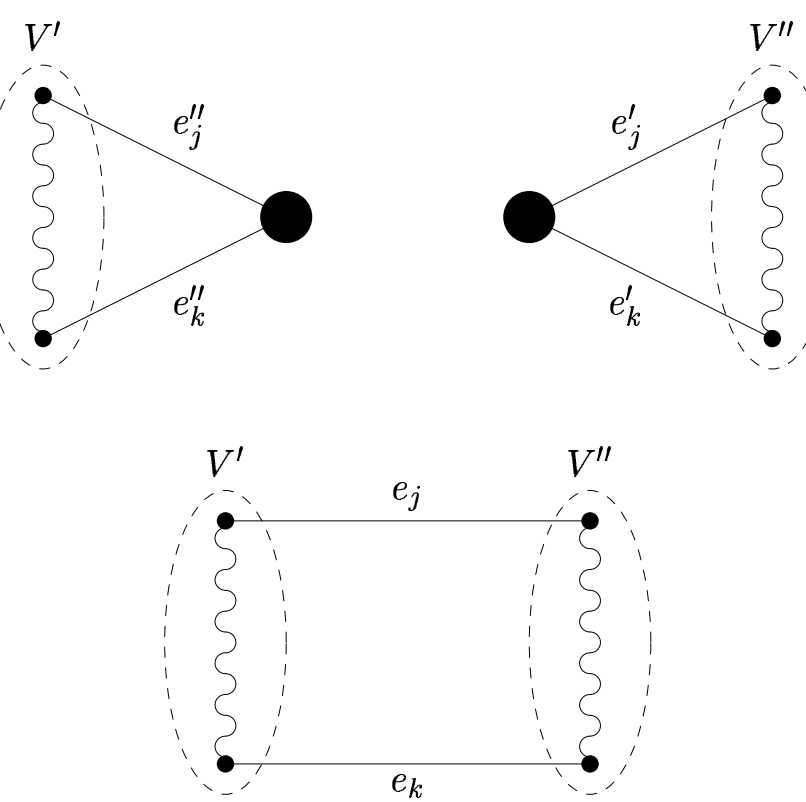

Figura 7.13: circuito em $G$ obtido a partir de um circuito em $\left(V / V^{\prime \prime}, E^{\prime \prime}\right)$ e um circuito em $\left(V / V^{\prime}, E^{\prime}\right)$.

Podemos concluir a partir da igualdade (7.20) e da formulação (7.22) que $d^{\prime}-\lambda_{i^{\prime}}^{\prime}$ satisfaz (7.21) somente se $c_{i^{\prime}}^{\prime}\left(e_{j}^{\prime}\right)=1$ e $c_{i^{\prime}}^{\prime}\left(\delta^{\prime}\left(V^{\prime \prime}\right) \backslash\left\{e_{j}^{\prime}, e_{k}^{\prime}\right\}\right)=0$. Portanto, demonstramos para todo $i^{\prime}$ que, se $C_{i^{\prime}}^{\prime} \cap \delta^{\prime}\left(V^{\prime \prime}\right) \neq \emptyset$, então $C_{i^{\prime}}^{\prime} \cap \delta^{\prime}\left(V^{\prime \prime}\right)$ contém exatamente duas arestas. Mais precisamente, esse conjunto contém $e_{j}^{\prime}$ e alguma aresta $e_{k}^{\prime}$ de $\delta^{\prime}\left(V^{\prime \prime}\right)$ tal que $k \neq j$.

Logo, se $k \neq j$, existem $\mu_{k, 1}^{\prime}, \ldots, \mu_{k, m_{k}^{\prime}}^{\prime}>0$ e circuitos $C_{k, 1}^{\prime}, \ldots, C_{k, m_{k}^{\prime}}^{\prime}$ em $\left(V / V^{\prime}, E^{\prime}\right)$ tais que:

- $\mu_{k, 1}^{\prime}+\cdots+\mu_{k, m_{k}^{\prime}}^{\prime}=d^{\prime}\left(e_{k}^{\prime}\right)$;

- $C_{k, i^{\prime}}^{\prime} \cap \delta^{\prime}\left(V^{\prime \prime}\right)=\left\{e_{j}^{\prime}, e_{k}^{\prime}\right\}$ para todo $i^{\prime}$

- $\mu_{k, 1}^{\prime} c_{k, 1}^{\prime}, \ldots, \mu_{k, m_{k}^{\prime}}^{\prime} c_{k, m_{k}^{\prime}}^{\prime} \in\left\{\lambda_{1}^{\prime} c_{1}^{\prime}, \ldots, \lambda_{m^{\prime}}^{\prime} c_{m^{\prime}}^{\prime}\right\}$, onde $c_{k, 1}^{\prime}, \ldots, c_{k, m_{k}^{\prime}}^{\prime}$ são os respectivos vetores de incidência de $C_{k, 1}^{\prime}, \ldots, C_{k, m_{k}^{\prime}}^{\prime}$.

Todos os argumentos utilizados podem ser aplicados de maneira análoga sobre $\left(V / V^{\prime \prime}, E^{\prime \prime}\right)$. Logo, se $k \neq j$, então existem $\mu_{k, 1}^{\prime \prime}, \ldots, \mu_{k, m_{k}^{\prime \prime}}^{\prime \prime}>0$ e circuitos $C_{k, 1}^{\prime \prime}, \ldots, C_{k, m_{k}^{\prime \prime}}^{\prime \prime}$ em $\left(V / V^{\prime \prime}, E^{\prime \prime}\right)$ tais que:

- $\mu_{k, 1}^{\prime \prime}+\cdots+\mu_{k, m_{k}^{\prime \prime}}^{\prime \prime}=d^{\prime \prime}\left(e_{k}^{\prime \prime}\right)$

- $C_{k, i^{\prime \prime}}^{\prime \prime} \cap \delta^{\prime \prime}\left(V^{\prime}\right)=\left\{e_{j}^{\prime \prime}, e_{k}^{\prime \prime}\right\}$ para todo $i^{\prime \prime}$;

- $\mu_{k, 1}^{\prime \prime} c_{k, 1}^{\prime \prime}, \ldots, \mu_{k, m_{k}^{\prime \prime}}^{\prime \prime} c_{k, m_{k}^{\prime \prime}}^{\prime \prime} \in\left\{\lambda_{1}^{\prime \prime} c_{1}^{\prime \prime}, \ldots, \lambda_{m^{\prime \prime}}^{\prime \prime} c_{m^{\prime \prime}}^{\prime \prime}\right\}$, onde $c_{k, 1}^{\prime \prime}, \ldots, c_{k, m_{k}^{\prime \prime}}^{\prime \prime}$ são os respectivos vetores de incidência de $C_{k, 1}^{\prime \prime}, \ldots, C_{k, m_{k}^{\prime \prime}}^{\prime \prime}$.

Podemos verificar que, a partir de qualquer circuito dentre $C_{k, 1}^{\prime}, \ldots, C_{k, m_{k}^{\prime}}^{\prime}$ e qualquer circuito dentre $C_{k, 1}^{\prime \prime}, \ldots, C_{k, m_{k}^{\prime \prime}}^{\prime \prime}$, podemos obter um circuito em $G$ através da união (7.19). Vamos então descrever como podemos obter uma combinação não-negativa dos circuitos de $G$ a partir de um alinhamento dos coeficientes $\mu_{k, 1}^{\prime}, \ldots, \mu_{k, m_{k}^{\prime}}^{\prime}$ e $\mu_{k, 1}^{\prime \prime}, \ldots, \mu_{k, m_{k}^{\prime \prime}}^{\prime \prime}$. 
Como as somas $\mu_{k, 1}^{\prime}+\cdots+\mu_{k, m_{k}^{\prime}}^{\prime}$ e $\mu_{k, 1}^{\prime \prime}+\cdots+\mu_{k, m_{k}^{\prime \prime}}^{\prime \prime}$ são ambas iguais a $d\left(e_{k}\right)$, podemos interpretar esses coeficientes como tamanhos das partes de uma partição do intervalo entre 0 e $d\left(e_{k}\right)$. Mais formalmente, considere dois conjuntos de reais $\alpha_{k, 0}^{\prime}, \alpha_{k, 1}^{\prime}, \ldots, \alpha_{k, m_{k}^{\prime}}^{\prime}$ e $\alpha_{k, 0}^{\prime \prime}, \alpha_{k, 1}^{\prime \prime}, \ldots, \alpha_{k, m_{k}^{\prime \prime}}^{\prime \prime}$ tais que

- $\alpha_{k, 0}^{\prime}=0$ e $\alpha_{k, i^{\prime}}^{\prime}=\alpha_{k, i^{\prime}-1}^{\prime}+\mu_{k, i^{\prime}}^{\prime}$ para todo $i^{\prime}>0$;

- $\alpha_{k, 0}^{\prime \prime}=0$ e $\alpha_{k, i^{\prime \prime}}^{\prime \prime}=\alpha_{k, i^{\prime \prime}-1}^{\prime \prime}+\mu_{k, i^{\prime \prime}}^{\prime \prime}$ para todo $i^{\prime \prime}>0$.

A partir dessa definição, sabemos que $\alpha_{k, i^{\prime}}^{\prime}-\alpha_{k, i^{\prime}-1}^{\prime}=\mu_{k, i^{\prime}}^{\prime}$ e $\alpha_{k, i^{\prime \prime}}^{\prime \prime}-\alpha_{k, i^{\prime \prime}-1}^{\prime \prime}=\mu_{k, i^{\prime \prime}}^{\prime \prime}$ para quaisquer $i^{\prime}, i^{\prime \prime} \geq 1$. Esses valores são os limitantes que determinam as partes. Sejam $\alpha_{k, 0}, \ldots, \alpha_{k, m_{k}}$ valores tais que $\alpha_{k, i-1}<\alpha_{k, i}$ para todo $i \geq 1 \mathrm{e}$

$$
\left\{\alpha_{k, 0}, \ldots, \alpha_{k, m_{k}}\right\}=\left\{\alpha_{k, 0}^{\prime}, \ldots, \alpha_{k, m_{k}^{\prime}}^{\prime}\right\} \cup\left\{\alpha_{k, 0}^{\prime \prime}, \ldots, \alpha_{k, m_{k}^{\prime \prime}}^{\prime \prime}\right\} .
$$

A figura 7.14 mostra um exemplo de como $\alpha_{k, 0}, \ldots, \alpha_{k, m_{k}}$ são obtidos através de um alinhamento.

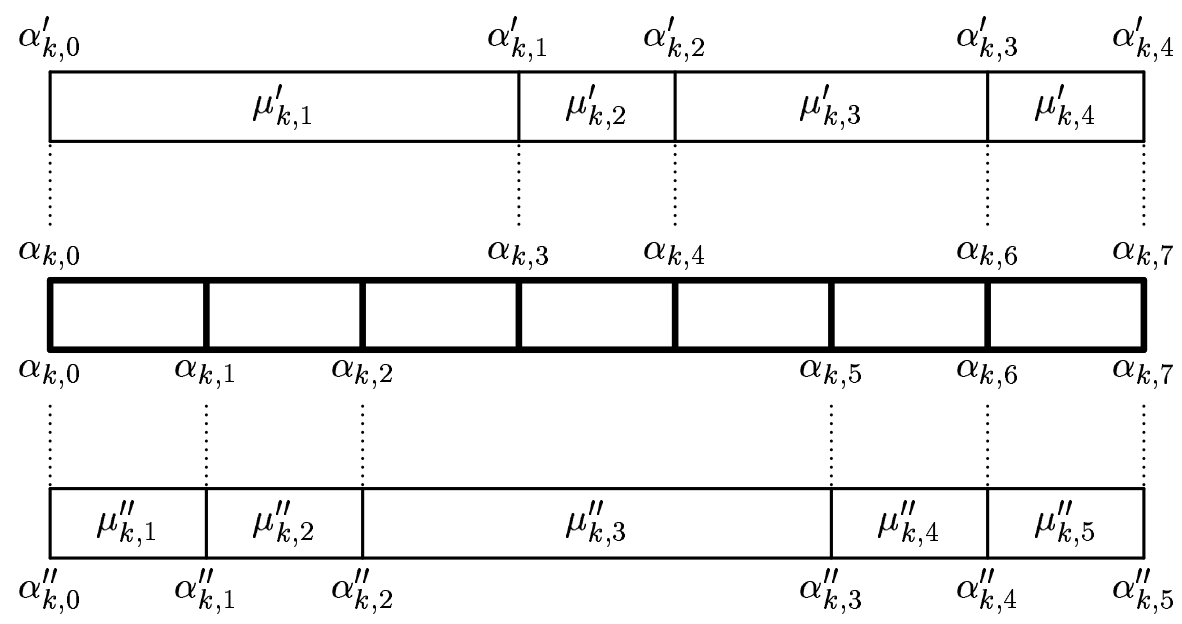

Figura 7.14: Exemplo de partição definida por dois conjuntos de coeficientes cuja soma é igual. Os valores dentro de cada intervalo indicam o comprimento.

Para todo $i \geq 1$, seja $\mu_{k, i}:=\alpha_{k, i}-\alpha_{k, i-1}$. Podemos verificar que existem $i^{\prime}, i^{\prime \prime} \geq 1$ tais que

- $\alpha_{k, i^{\prime}-1}^{\prime} \leq \alpha_{k, i-1} \leq \alpha_{k, i} \leq \alpha_{k, i^{\prime}}^{\prime}$

- $\alpha_{k, i^{\prime \prime}-1}^{\prime \prime} \leq \alpha_{k, i-1} \leq \alpha_{k, i} \leq \alpha_{k, i^{\prime \prime}}^{\prime \prime}$

Seja $C_{k, i}$ o circuito em $G$ obtido a partir de $C_{k, i^{\prime}}^{\prime}$ e $C_{k, i^{\prime \prime}}^{\prime \prime}$ através da união (7.19) e seja $c_{k, i}$ o vetor de incidência desse circuito. A partir dessa construção, podemos enfim verificar que $d$ é gerado por

$$
\left(c_{1}^{+}+\cdots+c_{r}^{+}\right)+\sum_{k}\left(\mu_{k, 1} c_{k, 1}+\cdots+\mu_{k, m_{k}} c_{k, m_{k}}\right) .
$$

A demonstração, no entanto, ainda não está concluída. Os argumentos partiram da hipótese de que $\left(V / V^{\prime}, E^{\prime}\right)$ e $\left(V / V^{\prime \prime}, E^{\prime \prime}\right)$ são grafos menores do que $G$. No entanto, isso não é necessariamente verdade. Vamos supor que estamos em um caso sobre o qual os argumentos anteriores não se aplicam:

- para todo $e \in E$, temos $d(e)>0$; 
- para todo $V^{\prime} \subseteq V$ e todo $e \in \delta\left(V^{\prime}\right)$ tais que $d(e)=d\left(\delta\left(V^{\prime}\right) \backslash\{e\}\right)$, temos $\left|V^{\prime}\right|=1$ ou $\left|V \backslash V^{\prime}\right|=1$

Vamos então estender a estratégia de redução para esse caso. Para dado $i$, sabemos que $\lambda_{d, i}^{*}>0$ se

$$
\frac{d\left(\delta\left(V^{\prime}\right) \backslash\{e\}\right)-d(e)}{c_{i}\left(\delta\left(V^{\prime}\right) \backslash\{e\}\right)-c_{i}(e)}>0
$$

para todo $V^{\prime} \subseteq V$ e todo $e \in \delta\left(V^{\prime}\right)$ tais que $c_{i}\left(\delta\left(V^{\prime}\right) \backslash\{e\}\right)-c_{i}(e) \neq 0$;

$$
\frac{d(e)}{c_{i}(e)}>0
$$

para todo $e \in E$ tal que $c_{i} \neq 0$.

Como estamos supondo que $d(e)>0$ para todo $e$, a segunda condição é satisfeita por qualquer $i$. Vamos demonstrar que podemos escolher $i$ de tal forma que, se $d\left(\delta\left(V^{\prime}\right) \backslash\{e\}\right)-d(e)=0$ para algum $V^{\prime} \subseteq V$ e $e \in \delta\left(V^{\prime}\right)$, então $c_{i}\left(\delta\left(V^{\prime}\right) \backslash\{e\}\right)-c_{i}(e)=0$. Para tal $i$, temos $\lambda_{d, i}^{*}>0$.

Estamos interessados em demonstrar que existe um circuito $C$ em $G$ tal que, para todo $V^{\prime} \subseteq V$ e todo $e \in \delta\left(V^{\prime}\right)$ tais que $d(e)=d\left(\delta\left(V^{\prime}\right) \backslash\{e\}\right)$, temos que $C$ não contém nenhuma aresta de $\delta\left(V^{\prime}\right)$ ou contém exatamente duas, sendo uma delas $e$. Estamos supondo que, se um subconjunto $V^{\prime}$ satisfaz a hipótese, então $\left|V^{\prime}\right|=1$ ou $\left|V \backslash V^{\prime}\right|=1$. Logo, já sabemos que $C$ sempre contém zero ou duas arestas desse $\delta\left(V^{\prime}\right)$. Resta demonstrar que, se contém duas, então contém $e$.

Suponha que $e$ seja uma aresta de $\delta\left(V^{\prime}\right)$ tal que $d(e)=d\left(\delta\left(V^{\prime}\right) \backslash\{e\}\right)$. Podemos verificar que, se $C$ é um circuito que contém uma aresta $f$ de $\delta\left(V^{\prime}\right)$ e $d(f)$ é máximo em $\delta\left(V^{\prime}\right)$, então $C$ contém $e$. Portanto, precisamos apenas demonstrar que existe em $G$ um circuito $C$ tal que, se $v$ é um vértice de $C$, então uma aresta $f$ em $\delta(v)$ com $d(f)$ máximo pertence a $C$.

Mais formalmente, seja $\varphi$ uma função que associa cada $v \in V$ tal que $\delta(v) \neq \emptyset$ a uma aresta $e$ de $\delta(v)$ tal que $d(e)$ é máximo. Se demonstrarmos que existe um circuito $C$ tal que para todo vértice $v$ de $C$ temos $\varphi(v) \in C$, podemos concluir que existe $i$ tal que $\lambda_{d, i}^{*}>0$. Tal demonstração pode ser obtida a partir do seguinte lema, provado por SEYmour [1979] em colaboração com Giles:

Lema 7.10. Seja $G=(V, E)$ um grafo e seja $\varphi$ uma função que associa cada $v \in V$ tal que $\delta(v) \neq \emptyset$ a alguma aresta em $\delta(v)$. Se $G$ contém pelo menos uma aresta e não contém pontes, então existe um circuito $C$ em $G$ tal que $\varphi(v) \in C$ para todo vértice $v$ desse circuito.

Demonstração. Vamos considerar uma indução em $|E|$. Como base da indução, podemos considerar o caso em que o grafo contém apenas duas arestas paralelas que formam o circuito desejado.

Seja $e=\left\{v_{1}, v_{2}\right\}$ uma aresta de $G$ e seja $\varphi\left(v_{2}\right)=f$. Vamos supor sem perda de generalidade que $\varphi\left(v_{1}\right)=e$. Se $f$ é uma aresta paralela a $e$, então as arestas $e, f$ formam o circuito desejado. Vamos então supor que $f=\left\{v_{2}, v_{3}\right\}$, onde $v_{3} \neq v_{1}$.

Seja $V^{\prime}:=\left\{v_{1}, v_{2}\right\}$, seja $\left(V / V^{\prime}, E^{\prime}\right)$ o grafo obtido a partir da contração de $V^{\prime}$, sejam $e_{1}, \ldots, e_{l}$ as arestas de $\delta\left(V^{\prime}\right)$ e sejam $e_{1}^{\prime}, \ldots, e_{l}^{\prime}$ suas respectivas arestas correspondentes. Em particular, seja $f^{\prime}$ a aresta correspondente a $f$. Vamos considerar então uma função $\varphi^{\prime}$ definida da seguinte forma:

$$
\varphi^{\prime}(v)= \begin{cases}f^{\prime} & \text { se } v=v^{\prime} \\ e_{i}^{\prime} & \text { se } v \neq v^{\prime}, \delta(v) \neq \emptyset \text { e } \delta(v)=e_{i} \\ \varphi(v) & \text { se } v \neq v^{\prime}, \delta(v) \neq \emptyset \text { e } \delta(v) \notin \delta\left(V^{\prime}\right) .\end{cases}
$$

Como $\left|E^{\prime}\right|<|E|$, podemos aplicar a hipótese de indução sobre $\left(V / V^{\prime}, E^{\prime}\right)$ e $\varphi^{\prime}$ e obter um circuito $C^{\prime}$ que satisfaz as propriedades desejadas para esse grafo e essa função. Substituindo nesse circuito 
as arestas que pertencem a $\left\{e_{1}^{\prime}, \ldots, e_{l}^{\prime}\right\}$ pelas respectivas arestas correspondentes em $\left\{e_{1}, \ldots, e_{l}\right\}$, obtemos um conjunto de arestas de $G$ que forma o circuito desejado por si próprio ou forma o circuito desejado quando adicionamos a ele a aresta $f$.

Resta considerar o caso em que, se $e=\left\{v_{1}, v_{2}\right\}$ e $\varphi\left(v_{1}\right)=e$, então $\varphi\left(v_{2}\right)=e$. Mas nesse caso,

$$
M:=\{e \in E: \text { existe } v \in V \text { tal que } \varphi(v)=e\}
$$

é um emparelhamento que cobre todos os vértices não-isolados. Como $G$ não tem pontes, segue que existe um circuito $M$-alternante em $G$. Esse circuito alternante satisfaz a propriedade desejada.

Vamos então recapitular todos os argumentos anteriores para concluir, enfim, a demonstração.

Teorema 7.11. Seja $G=(V, E)$ um grafo e seja c um vetor indexado pelas arestas desse grafo. $O$ vetor c pertence ao cone dos circuitos de $G$ se e somente se c é não-negativo e, para todo $V^{\prime} \subseteq V e$ todo $e \in \delta\left(V^{\prime}\right)$, temos $c(e) \leq c\left(\delta\left(V^{\prime}\right) \backslash\{e\}\right)$.

Demonstração. Vamos considerar uma indução em $|E|$ e vamos considerar como base da indução o caso em que $|E|=0$. Seja $d:=c-\lambda_{c, i}^{*} c_{i}$ para algum $i$. Se $d(e)=0$ para algum $e$, podemos demonstrar a afirmação aplicando a hipótese de indução sobre uma restrição de $d$. Se existe $V^{\prime} \subseteq V$ e $e \in \delta\left(V^{\prime}\right)$ tais que $d(e)=d\left(\delta\left(V^{\prime}\right) \backslash\{e\}\right)$ e pelo menos um dentre $V^{\prime}$ e $V \backslash V^{\prime}$ contém mais de um vértice, podemos demonstrar a afirmação aplicando a hipótese de indução sobre os grafos obtidos a partir da contração de $V^{\prime}$ e $V \backslash V^{\prime}$. Se nenhum desses casos se aplica, existe $i$ tal que $\lambda_{d, i}^{*}>0$. Como o número de arestas e o número de subconjuntos de $V$ são ambos finitos, uma seqüência de reduções eventualmente conduz a um dos casos sobre os quais podemos reduzir o grafo e aplicar indução.

Demonstramos então caracterizações combinatórias para o cone e para o reticulado dos circuitos. Naturalmente, podemos considerar como próximo passo uma caracterização para o cone inteiro.

\section{Cone inteiro dos circuitos}

O cone inteiro gerado pelos circuitos de um grafo é por definição um subconjunto do fecho linear, do reticulado e do cone dos circuitos. Todas as condições que foram apresentadas nesta seção, portanto, são condições necessárias para que um vetor pertença a esse conjunto gerado. Podemos então conjecturar que essas condições, quando consideradas simultaneamente, são suficientes.

Conjectura 7.12. Seja $G=(V, E)$ um grafo e seja c um vetor indexado pelas arestas desse grafo. $O$ vetor c pertence ao cone inteiro dos circuitos de $G$ se e somente se c é inteiro não-negativo, $c$ atribui custo par aos cortes de $G$ e, para todo $V^{\prime} \subseteq V$ e todo $e \in \delta\left(V^{\prime}\right)$, temos $c(e) \leq c\left(\delta\left(V^{\prime}\right) \backslash\{e\}\right)$.

Sabemos então que essa conjectura é verdadeira se e somente se os vetores de incidência dos circuitos de qualquer grafo formam uma base de Hilbert.

Antes de entrarmos em mais detalhes a respeito dessa conjectura, vamos considerar alguns casos particulares. Suponha que estamos interessados em verificar se um vetor especifico pertence ao cone inteiro dos circuitos de um grafo. Um exemplo de problema combinatório que pode ser formulado dessa maneira é verificar se o conjunto de arestas de um grafo pode ser particionado em circuitos. Tal partição existe se e somente se o vetor $(1, \ldots, 1)$ é gerado por uma combinação inteira não-negativa dos circuitos desse grafo. A figura 7.15 mostra uma partição em circuitos.

Se o vetor $(1, \ldots, 1)$ pertence ao cone inteiro dos circuitos de um grafo, então podemos verificar que esse cone inteiro também contém o vetor $(k, \ldots, k)$ para qualquer inteiro não-negativo $k$. 


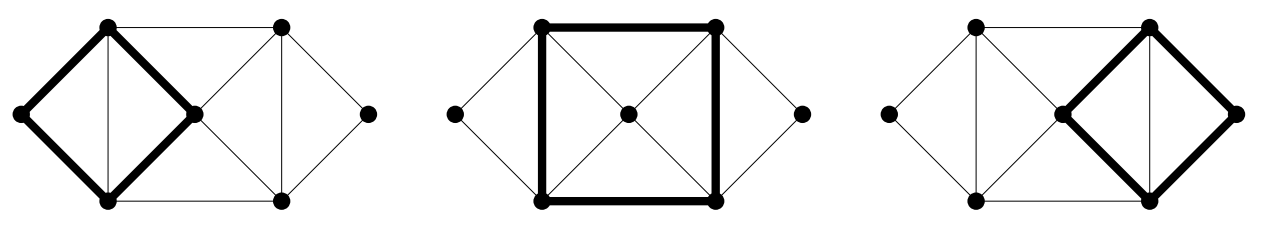

Figura 7.15: exemplo de grafo cujo conjunto de arestas pode ser particionado em circuitos.

Porém, a pertinência de $(1, \ldots, 1)$ não é necessária. Considere, por exemplo, o caso em que $k=2$. Sabemos que $(2, \ldots, 2)$ pertence ao cone dos circuitos se e somente se existe um conjunto de circuitos tal que toda aresta pertence a exatamente dois circuitos do conjunto. Dizemos que tal conjunto é uma cobertura dupla por circuitos. A figura 7.16 mostra um exemplo de cobertura dupla.
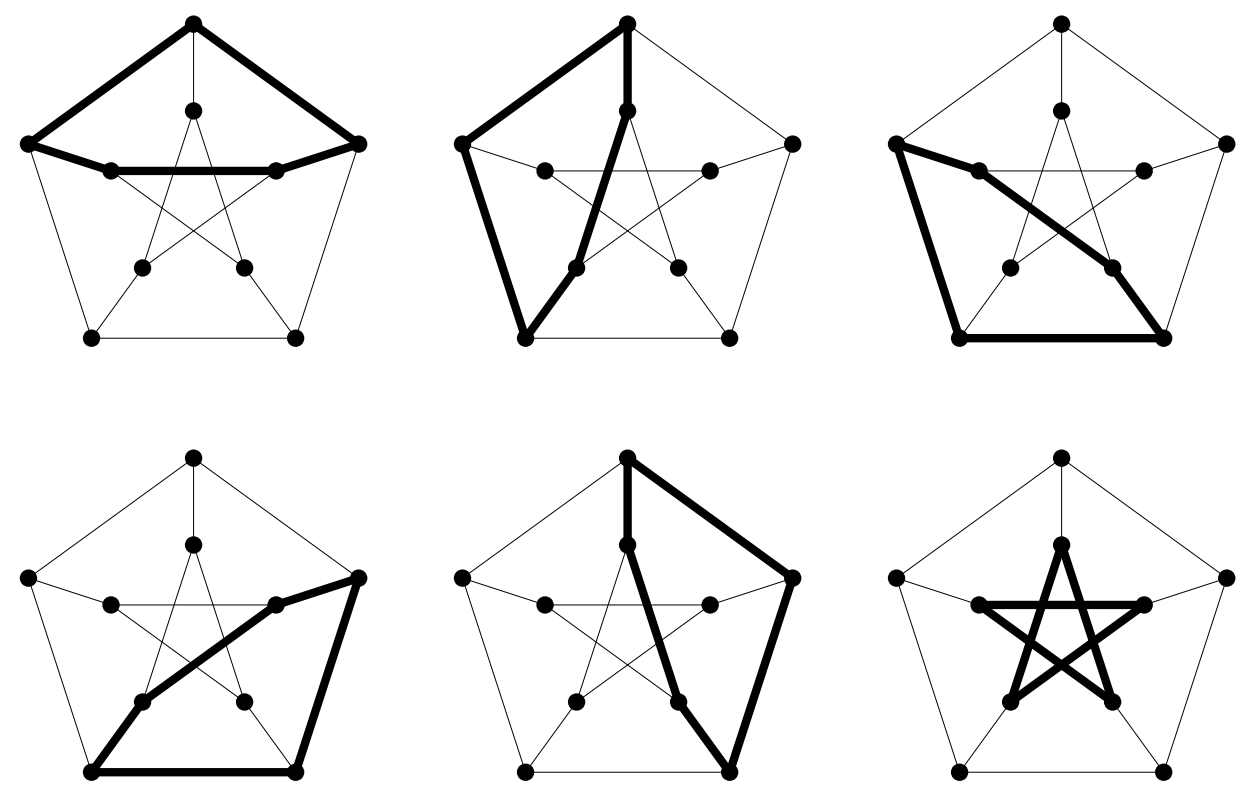

Figura 7.16: cobertura dupla por circuitos do grafo de Petersen.

Podemos verificar que o conjunto de arestas do grafo ao qual esse exemplo se refere não pode ser particionado em circuitos, pois os graus dos vértices desse grafo são ímpares. Portanto, a existência de uma partição em circuitos não é necessária para existência de uma cobertura dupla.

SzEKEREs [1973] e SeYmour [1979] conjecturaram que a não-existência de pontes é uma condição suficiente para que uma cobertura dupla por circuitos exista. Essa é uma das conjecturas mais conhecidas em teoria dos grafos e até o momento foi demonstrada apenas para casos particulares.

Conjectura 7.13. Seja $G$ um grafo. Se $G$ não tem pontes, então existe um conjunto de circuitos de $G$ tal que cada aresta de $G$ pertence a exatamente dois circuitos do conjunto.

Podemos verificar que, se o grafo não tem pontes, então o vetor $(2, \ldots, 2)$ é admissível e atribui custo par aos cortes. Logo, podemos concluir que a conjectura 7.13 é verdadeira se demonstrarmos que a conjectura 7.12 é verdadeira. No entanto, existe um contra-exemplo para a conjectura 7.12. A demonstração por contagem que será apresentada a seguir foi baseada em um texto de MURTY [1994]: 
Teorema 7.14. Existe um grafo $G$ e um vetor $c$ indexado pelas arestas desse grafo tais que c pertence ao cone e ao reticulado dos circuitos de $G$ mas c não pertence ao cone inteiro dos circuitos de $G$.

Demonstração. Considere o grafo de Petersen e a função de custos da figura 7.17. As combinações vistas na figura 7.18 demonstram que essa função pertence ao cone e ao reticulado dos circuitos.

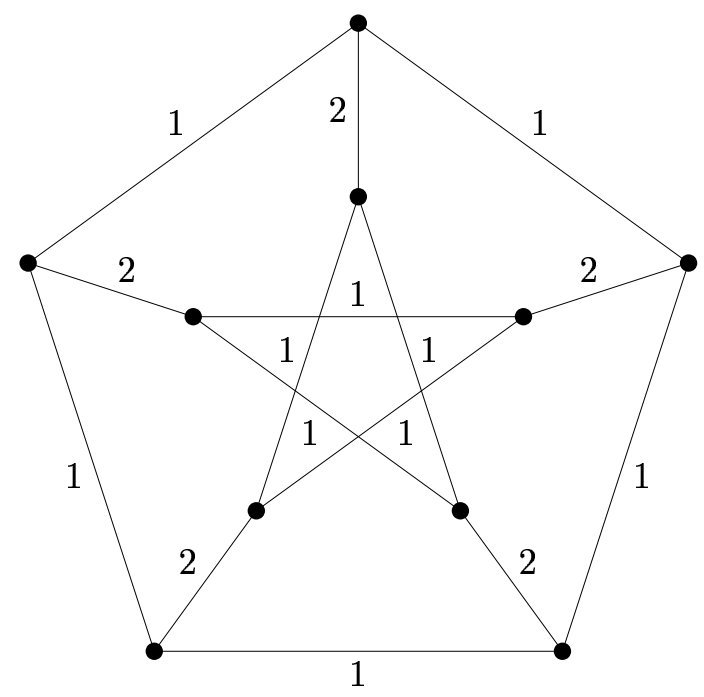

Figura 7.17: exemplo de função de custos que, embora pertença ao cone e ao reticulado dos circuitos, não pertence ao cone inteiro.
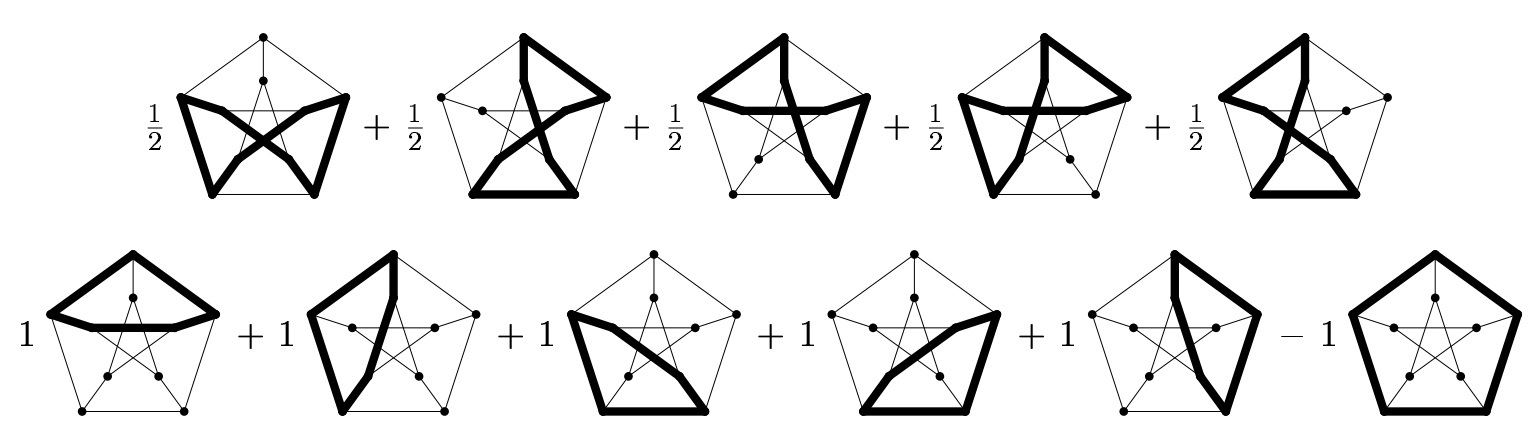

Figura 7.18: Acima, uma combinação não-negativa de circuitos que gera a função de custos da figura 7.17. Abaixo, uma combinação inteira de circuitos que gera essa função.

Suponha essa função de custos é gerada por uma soma de circuitos. Qualquer circuito do grafo que contém uma das arestas de custo 2 também contém uma aresta do pentágono. Logo, podemos verificar que um circuito que faz parte da soma contém no máximo uma aresta do pentágono. Por outro lado, podemos verificar também que, se um circuito contém apenas uma aresta do pentágono, então esse circuito contém duas arestas do pentagrama. Temos uma contradição, pois cada aresta do pentágono e cada aresta do pentagrama pode pertencer a no máximo um circuito da soma.

SEYMOUR [1979] demonstrou que as duas conjecturas são verdadeiras para o caso particular em que o grafo considerado é planar. AlsPaCH, GODDYN E ZHANG [1994] estenderam esse resultado e demonstraram as conjecturas para o caso em que o grafo não contém um menor de Petersen. 


\subsection{Florestas maximais e árvores geradoras}

Novamente, seja $G=(V, E)$ um grafo. Para encerrar este capítulo, vamos considerar os vetores de incidência das florestas de $G$. Como um conjunto formado por uma única aresta é um caso particular de floresta, existem caracterizações muito simples para o fecho linear, o reticulado, o cone e o cone inteiro das florestas. Se $x$ é um vetor real indexado pelas arestas de $G$, sabemos que:

- $x$ pertence ao fecho linear das florestas;

- $x$ pertence ao reticulado das florestas se e somente se $x$ é inteiro;

- $x$ pertence ao cone das florestas se e somente se $x$ é não-negativo;

- $x$ pertence ao cone inteiro das florestas se e somente se $x$ é inteiro e não-negativo;

Esses quatro conjuntos gerados, portanto, não são muito interessantes se todas as florestas fazem parte do conjunto gerador. Vamos então considerar nesta seção um conjunto gerador mais específico: as florestas maximais de $G$. Em particular, se esse grafo é conexo, essas florestas maximais são as suas árvores geradoras. A figura 7.19 mostra um exemplo de combinação de árvores geradoras.

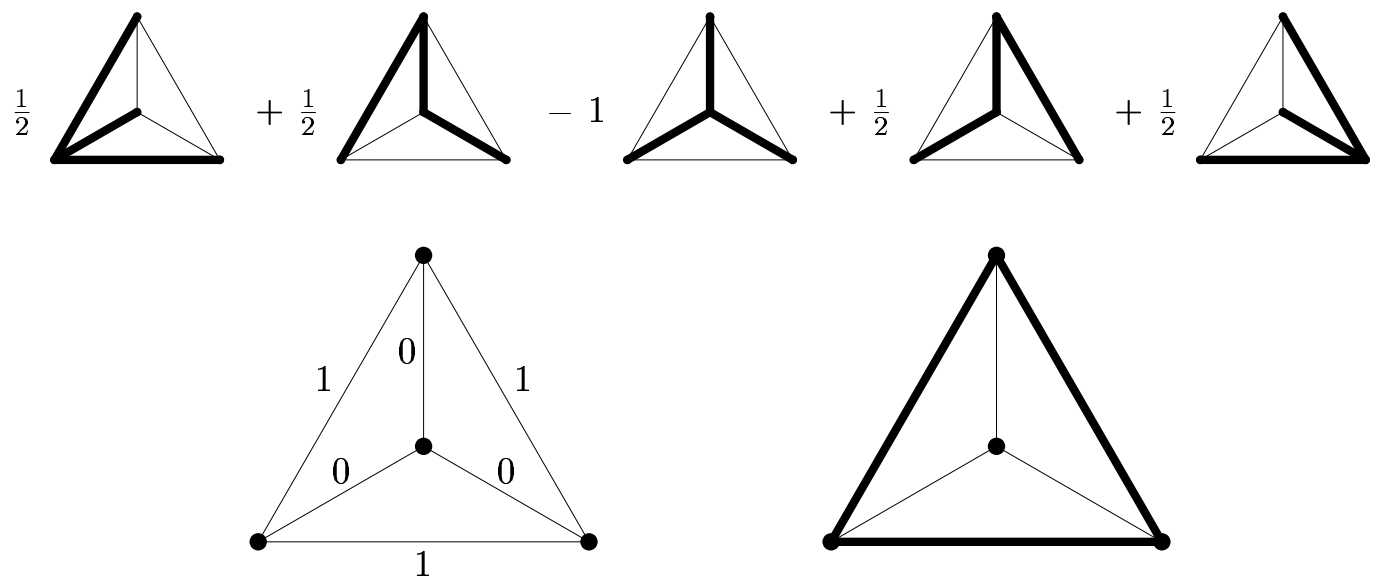

Figura 7.19: Uma combinação linear de árvores geradoras e a função de custos gerada por essa combinação. Neste caso em particular, a função gerada é o vetor de incidência de um circuito.

Mas antes de nos aprofundarmos em florestas maximais, vamos apresentar um conjunto gerado por todas as florestas que, diferente dos quatro primeiros, possui uma caracterização não-trivial.

\section{Fecho convexo das florestas}

Para todo subconjunto $E^{\prime}$ de $E$, vamos denotar por $r\left(E^{\prime}\right)$ a cardinalidade máxima de uma floresta de $G$ contida em $E^{\prime}$. Segue dessa definição que, se $x$ é o vetor de incidência de uma floresta de $G$,

$$
\begin{array}{rlrl}
x\left(E^{\prime}\right) & \leq r\left(E^{\prime}\right) & & \text { para todo } E^{\prime} \subseteq E ; \\
x(e) \geq 0 & & \text { para todo } e \in E .
\end{array}
$$

Embora essas duas condições sejam bastante simples, EDMONDs [1970] demonstrou que elas são suficientes para caracterizar o fecho convexo das florestas. Vamos apresentar uma demonstração poliédrica dessa caracterização, baseada no livro de Grötschel, LovÁsz E SchriJver [1993]. 
Teorema 7.15. Seja $G=(V, E)$ um grafo e seja $x$ um vetor indexado pelas arestas desse grafo. $O$ vetor $x$ pertence ao fecho convexo das florestas de $G$ se e somente se $x$ é não-negativo e $x\left(E^{\prime}\right) \leq r\left(E^{\prime}\right)$ para todo $E^{\prime} \subseteq E$.

Demonstração. Pelo corolário 2.25, sabemos que o fecho convexo das florestas é dado por um poliedro

$$
\mathcal{P}:=\{x: A x \leq b\}
$$

Sem perda de generalidade, vamos supor que o sistema $A x \leq b$ é minimal em relação a $\mathcal{P}$. Como sabemos que $\{e\}$ é uma floresta para todo $e \in E$ e que o conjunto vazio é um caso particular de floresta, podemos verificar pelo teorema 2.13 que esse poliedro tem dimensão plena.

Seja $\mathcal{Q}:=\left\{x: x \geq 0\right.$ e $x\left(E^{\prime}\right) \leq r\left(E^{\prime}\right)$ para todo $\left.E^{\prime} \subseteq E\right\}$. Por definição, sabemos que $\mathcal{P} \subseteq \mathcal{Q}$. Pelo corolário 2.17, podemos concluir a demonstração se provarmos que para toda restrição $a x \leq \beta$ de $A x \leq b$ vale uma das seguintes afirmações:

(i) $\{x \in \mathcal{P}: a x=\beta\} \subseteq\{x \in \mathcal{P}: x(e)=0\}$ para algum $e \in E$;

(ii) $\{x \in \mathcal{P}: a x=\beta\} \subseteq\left\{x \in \mathcal{P}: x\left(E^{\prime}\right)=r\left(E^{\prime}\right)\right\}$ para algum $E^{\prime} \subseteq E$.

Seja $a x \leq \beta$ uma restrição de $A x \leq b$. Primeiramente, vamos supor que existe $e$ tal que $a(e)<0$. Seja $F$ uma floresta de $G$, seja $x_{F}$ o vetor de incidência dessa floresta e suponha que $a x_{F}=\beta$. Vamos provar que $x_{F}(e)=0$. Suponha por contradição que $x_{F}(e)>0$. Nesse caso, temos que $e \in F \mathrm{e}$ que $F \backslash\{e\}$ é uma outra floresta. Logo, se $x^{\prime}$ é o vetor de incidência de $F \backslash\{e\}$, então temos que

$$
a x^{\prime}=a x_{F}-a(e)>a x_{F}=\beta .
$$

Mas isso é uma contradição, pois o vetor $x^{\prime}$ pertence ao poliedro $\mathcal{P}$ e, portanto, deveria ser verdade que $a x^{\prime} \leq \beta$. Portanto, como $\mathcal{P}$ é o fecho convexo das florestas, vale a afirmação (i).

Vamos supor agora que $a(e) \geq 0$ para todo $e$. Como $A x \leq b$ é minimal, sabemos que o conjunto

$$
E^{\prime}:=\{e \in E: a(e)>0\}
$$

é não-vazio. Novamente, seja $F$ uma floresta, seja $x_{F}$ o vetor de incidência dessa floresta e suponha que $a x_{F}=\beta$. Vamos provar que $x_{F}\left(E^{\prime}\right)=r\left(E^{\prime}\right)$. Suponha por contradição que $x_{F}\left(E^{\prime}\right)<r\left(E^{\prime}\right)$. Nesse caso, existe $e \in E^{\prime}$ tal que $F \cup\{e\}$ é uma floresta. Logo, se $x^{\prime \prime}$ é o vetor de incidência de $F \cup\{e\}$,

$$
a x^{\prime \prime}=a x_{F}+a(e)>a x_{F}=\beta .
$$

Novamente temos uma contradição e, pelos mesmos argumentos anteriores, segue que (ii) vale.

Vamos agora entrar em mais detalhes a respeito da função $r$. Sabemos que, para todo $E^{\prime} \subseteq E$, o valor de $r\left(E^{\prime}\right)$ é $|V|-k$, onde $k$ é o número de componentes do grafo $\left(V, E^{\prime}\right)$.

$\mathrm{Na}$ verdade, podemos generalizar esse argumento para qualquer conjunto de vértices que contém as arestas em $E^{\prime}$. Se $V^{\prime} \subseteq V$ e $E^{\prime} \subseteq E$ é um conjunto de arestas contidas em $V^{\prime}$, então temos que $r\left(E^{\prime}\right)=\left|V^{\prime}\right|-k^{\prime}$, onde $k^{\prime}$ é o número de componentes de $\left(V^{\prime}, E^{\prime}\right)$.

A partir dessas relações entre conjuntos de arestas e conjuntos de vértices, podemos reformular a caracterização do fecho convexo das florestas em termos de conjuntos de vértices:

Corolário 7.16. Seja $G=(V, E)$ um grafo e seja $x$ um vetor indexado pelas arestas desse grafo. $O$ vetor $x$ pertence ao fecho convexo das florestas de $G$ se e somente se $x\left(V^{\prime}\right) \leq\left|V^{\prime}\right|-1$ para todo $V^{\prime} \subseteq V$ e $x$ é não-negativo. 
Demonstração. Primeiramente, suponha que $x\left(E^{\prime}\right) \leq r\left(E^{\prime}\right)$ para todo $E^{\prime} \subseteq E$. Seja $V^{\prime} \subseteq V$ e seja $E^{\prime}$ o conjunto das arestas de $G$ que estão contidas em $V^{\prime}$. Temos então por definição que

$$
x\left(V^{\prime}\right)=x\left(E^{\prime}\right) \leq r\left(E^{\prime}\right) \leq\left|V^{\prime}\right|-k^{\prime}, \text { onde } k^{\prime} \geq 1 .
$$

Suponha agora que $x\left(V^{\prime}\right) \leq\left|V^{\prime}\right|-1$ para todo $V^{\prime} \subseteq V$. Seja $E^{\prime} \subseteq E$ e sejam $\left(V_{1}, E_{1}^{\prime}\right), \ldots,\left(V_{k}, E_{k}^{\prime}\right)$ as componentes de $\left(V, E^{\prime}\right)$. Sabemos por definição que $x\left(E^{\prime}\right) \leq x\left(V_{1}\right)+\cdots+x\left(V_{k}\right)$. Portanto,

$$
x\left(E^{\prime}\right) \leq\left(\left|V_{1}\right|-1\right)+\cdots+\left(\left|V_{k}\right|-1\right)=|V|-k=r\left(E^{\prime}\right) .
$$

Portanto, concluímos que as condições consideradas equivalem às condições do teorema 7.15.

Não serão apresentados neste texto outros resultados a respeito de conjuntos gerados por todas as florestas. Porém, essa caracterização do fecho convexo será útil em demonstrações posteriores.

\section{Reticulado das árvores geradoras}

A partir deste ponto, vamos considerar conjuntos gerados por florestas maximais ao invés de florestas arbitrárias. Em particular, vamos supor que $G$ é um grafo conexo e, portanto, que essas florestas maximais são as árvores geradoras do grafo.

Sejam $T_{1}, \ldots, T_{m}$ as árvores geradoras de $G$ e sejam $t_{1}, \ldots, t_{m}$ os seus respectivos vetores de incidência. Uma propriedade particularmente interessante a respeito de árvores geradoras é o fato de que, diferente dos circuitos, todas elas possuem a mesma cardinalidade. Mais formalmente,

para todo $i$, temos $t_{i}(E)=|V|-1$.

Portanto, a soma dos custos de um vetor gerado por uma combinação inteira de árvores geradoras é um múltiplo de $|V|-1$. Segue naturalmente desse fato uma condição necessária para que um dado vetor $t$ pertença ao reticulado das árvores geradoras:

se um vetor $t$ pertence ao reticulado das árvores geradoras de $G$, então $t$ é um vetor inteiro e $t(E) /(|V|-1)$ é um número inteiro.

Podemos verificar, no entanto, que essa condição não é suficiente. A figura 7.20 demonstra essa insuficiência através de um exemplo no qual o grafo considerado possui apenas uma árvore geradora.

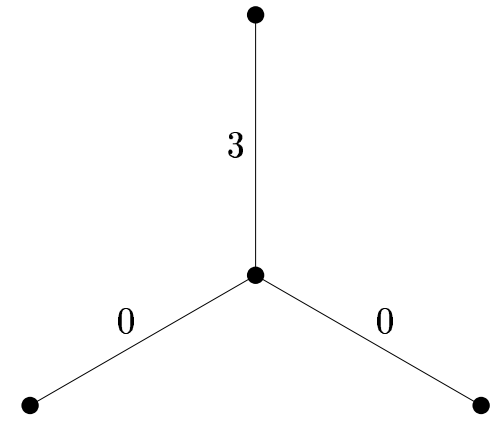

Figura 7.20: Exemplo de função de custos tal que a soma total dos custos é múltiplo de $|V|-1$. Essa função não pode ser gerada pela única árvore geradora.

Uma das principais desvantagens do grafo apresentado nesse exemplo é a ausência de circuitos. Como veremos a seguir, circuitos são particularmente úteis para caracterizarmos o reticulado. 
Suponha que no grafo $G$ existe um circuito $C$. Se $e$ é uma aresta desse circuito, sabemos por definição que $C \backslash\{e\}$ é uma floresta. Ademais, sabemos que essa floresta está contida em alguma árvore geradora $T^{\prime}$, pois o grafo é conexo. Podemos verificar então que, se $f$ é uma aresta de $C$ e

$$
T^{\prime \prime}:=\left(T^{\prime} \cup\{e\}\right) \backslash\{f\},
$$

então $T^{\prime \prime}$ também é uma árvore geradora. Portanto, se $e \neq f$ e $t^{\prime}, t^{\prime \prime}$ são os respectivos vetores de incidência de $T^{\prime}, T^{\prime \prime}$, então o vetor $d:=t^{\prime}-t^{\prime \prime}$ pertence ao reticulado das árvores geradoras d $G$ e

$d$ atribui -1 à aresta e, atribui 1 à aresta $f$ e atribui 0 às arestas restantes.

Dizemos que $d$ é um vetor de transferência, pois a adição desse vetor transfere custo de e para $f$ :

$$
t(e)+k d(e)=t(e)-k \quad \text { e } \quad t(f)+k d(f)=t(f)+k
$$

para todo vetor $t$ indexado pelas arestas. A figura 7.21 mostra um exemplo de vetor de transferência.
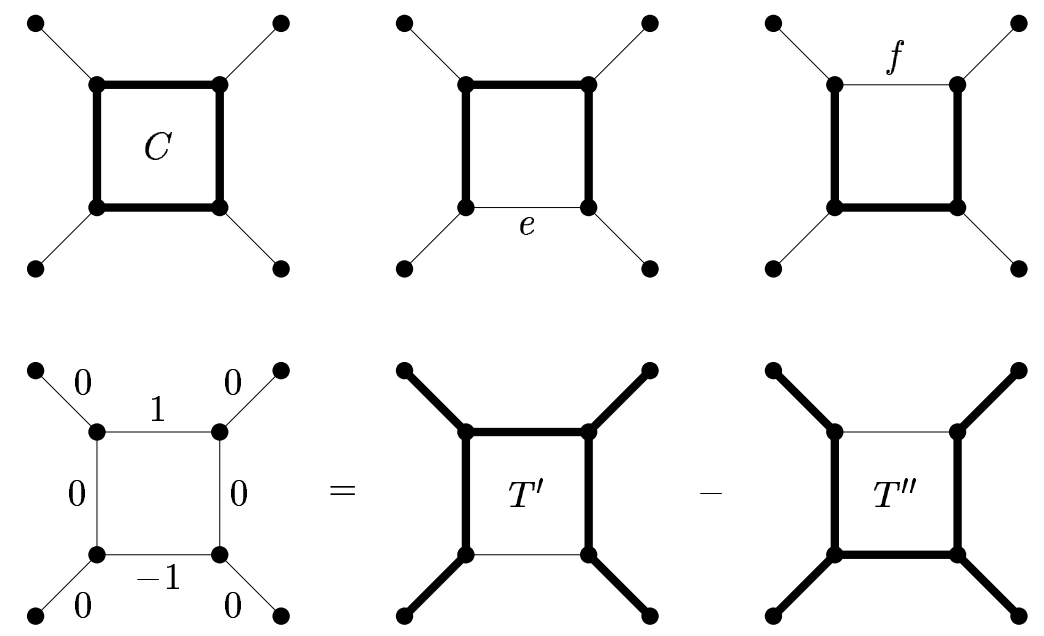

Figura 7.21: Acima, um circuito e florestas obtidas quando retiramos arestas desse circuito. Abaixo, a diferença entre duas árvores geradoras que contém essas florestas.

Tais vetores são escolhas interessantes para uma estratégia de redução, pois eles pertencem ao reticulado das árvores geradoras e também não alteram a soma dos custos quando adicionados:

$$
\sum_{e \in E}(t(e)+d(e))=\sum_{e \in E} t(e)
$$

A adição de vetores de transferência pode ser utilizada para redistribuir o custo total entre as arestas do grafo. Suponha que, para quaisquer arestas distintas $e, f$, existe um vetor de transferência. Podemos verificar nesse caso que, se $t$ e $p$ são vetores inteiros tais que $t(E) /(|V|-1)$ e $p(E) /(|V|-1)$ são ambos iguais a um inteiro $\lambda$, então existem vetores de transferência $d_{1}, \ldots, d_{l}$ para os quais

$$
p=t+d_{1}+\cdots+d_{l} .
$$

Em particular, suponha que existe uma árvore geradora $T$ em $G$ tal que $p(e)=\lambda$ se $e \in T$ e $p(e)=0$ caso contrário. Nesse caso, podemos verificar que o vetor $p$ pertence ao reticulado das árvores geradoras e a igualdade (7.23) garante que o vetor $t$ também pertence a esse reticulado. 
Esses argumentos partem da hipótese de que existe um vetor de transferência para qualquer par de arestas distintas. Embora nem todos os grafos conexos satisfaçam essa hipótese, podemos considerar a estratégia de aplicar os argumentos sobre subgrafos de $G$ que a satisfazem.

Seja $\left(V^{\prime}, E^{\prime}\right)$ um subgrafo de $G$. Dizemos que esse subgrafo é um bloco do grafo conexo $G$ se para todo $v \in V^{\prime}$, o subgrafo de $\left(V^{\prime}, E^{\prime}\right)$ induzido por $V^{\prime} \backslash\{v\}$ é conexo e $\left(V^{\prime}, E^{\prime}\right)$ é maximal. Essa maximalidade garante que, se $\left(V_{1}, E_{1}\right), \ldots,\left(V_{l}, E_{l}\right)$ são os blocos de $G$,

- $\left|V_{i}\right| \geq 2$ e $\left|E_{i}\right| \geq 1$ para todo $i$

- $\left\{E_{1}, \ldots, E_{l}\right\}$ é uma partição de $E$;

- $T \subseteq E$ é uma árvore geradora de $G$ se e somente se existem $T_{1}, \ldots, T_{l} \subseteq E$ tais que $T_{i}$ é uma árvore geradora de $\left(V_{i}, E_{i}\right)$ para todo $i$ e $T=\bigcup_{i=1}^{l} T_{i}$,

O lema apresentado a seguir esclarece porque esse conceito de blocos é útil no presente contexto.

Lema 7.17. Seja $G$ um grafo conexo e seja $\left(V_{i}, E_{i}\right)$ um bloco desse grafo. Se $\left|E_{i}\right| \geq 2$, então para quaisquer $e, f \in E_{i}$ tais que $e \neq f$ existe um circuito $C$ em $\left(V_{i}, E_{i}\right)$ tal que $e, f \in C$.

Demonstração. Sabemos que $\left|V_{i}\right| \geq 2$. Se $\left|V_{i}\right|=2$, então as arestas em $E_{i}$ são mutuamente paralelas e, portanto, qualquer par de arestas distintas forma um circuito. Vamos então supor $\left|V_{i}\right| \geq 3$.

Sejam $u, v$ vértices distintos em $V_{i}$. Pela definição de bloco sabemos que, se $V_{i}^{\prime} \subseteq V_{i} \backslash\{u, v\}$ é tal que $\left(V_{i} \backslash V_{i}^{\prime}, E_{i}\right)$ não contém $(u, v)$-caminhos, então $\left|V_{i}^{\prime}\right| \geq 2$. Logo, pelo teorema de Menger, existem dois $(u, v)$-caminhos disjuntos em $\left(V_{i}, E_{i}\right)$. Portanto, se considerarmos esse fato em relação aos vértices que pertencem a duas arestas distintas $e, f$, podemos obter o circuito $C$ desejado.

Assim, para obtermos uma caracterização do reticulado das árvores geradoras, precisamos apenas estender para todos os blocos do grafo a condição necessária que foi mencionada anteriormente.

Teorema 7.18. Seja $G=(V, E)$ um grafo conexo, seja $t$ um vetor indexado pelas arestas desse grafo e seja $\lambda:=t(E) /(|V|-1)$. O vetor $t$ pertence ao reticulado das árvores geradoras de $G$ se e somente se $t$ é um vetor inteiro, $\lambda$ é um número inteiro e, para todo bloco $\left(V^{\prime}, E^{\prime}\right)$ de $G$, temos $t\left(E^{\prime}\right)=\lambda\left(\left|V^{\prime}\right|-1\right)$.

Demonstração. Sabemos pelo lema 7.17 que, para quaisquer duas arestas distintas de um bloco com duas ou mais arestas, existe um vetor de transferência. Logo, se $p$ é um vetor que também satisfaz as hipóteses sobre $t$, então existem vetores de transferência $d_{1}, \ldots, d_{l}$ para os quais a igualdade (7.23) vale. Em particular, podemos supor que existe uma árvore geradora $T$ de $G$ tal que $p(e)=\lambda$ se $e \in T$ e $p(e)=0$ caso contrário. Nesse caso, $p, t$ pertencem ambos ao reticulado das árvores geradoras.

O fato de que a soma total dos custos de um vetor que pertence ao reticulado das árvores geradoras é um múltiplo de $|V|-1$ será considerado novamente em demonstrações posteriores.

\section{Cone das árvores geradoras}

Vamos apresentar a seguir uma caracterização para o cone das árvores geradoras. A demonstração que será exibida é consideravelmente mais simples que a do cone dos circuitos. Pode-se dizer que essa simplicidade é conseqüência do fato de que ela pode ser obtida naturalmente a partir de um conjunto gerado que não tínhamos quando estávamos trabalhando com circuitos: o fecho convexo. 
Teorema 7.19. Seja $G=(V, E)$ um grafo e seja $t$ um vetor indexado pelas arestas desse grafo. $O$ vetor $t$ pertence ao fecho convexo das árvores geradoras de $G$ se e somente se $t\left(V^{\prime}\right) \leq\left|V^{\prime}\right|-1$ para todo $V^{\prime} \subseteq V$, $t$ é não-negativo e $t(V)=|V|-1$.

Demonstração. Sabemos que um subconjunto $T$ de $E$ é uma árvore geradora de $G$ se e somente se $T$ é uma floresta e $|T|=|V|-1$. Sabemos também que, para toda floresta $F$ de $G$, temos $|F| \leq|V|-1$. Podemos verificar, portanto, que a caracterização desejada segue diretamente do corolário 7.16.

Suponha então que $t$ é um vetor no cone das árvores geradoras. Mais formalmente, suponha que

$$
t=\lambda_{1} t_{1}+\cdots+\lambda_{m} t_{m}
$$

onde $\lambda_{1}, \ldots, \lambda_{m}$ são números não-negativos. Se $\lambda:=\sum_{i=1}^{m} \lambda_{i}$, então temos que a combinação linear

$$
\frac{\lambda_{1}}{\lambda} t_{1}+\cdots+\frac{\lambda_{m}}{\lambda} t_{m}
$$

é uma combinação convexa. Por outro lado, sabemos que qualquer combinação convexa multiplicada por um número não-negativo é uma combinação não-negativa.

Portanto, um vetor $t$ pertence ao cone se e somente se existe $\lambda \geq 0$ e $t^{\prime}$ no fecho convexo tal que $t=\lambda t^{\prime}$. Desse fato segue naturalmente uma caracterização para o cone das árvores geradoras.

Teorema 7.20. Seja $G=(V, E)$ um grafo, seja $t$ um vetor indexado pelas arestas desse grafo $e$ seja $\lambda:=t(V) /(|V|-1)$. O vetor $t$ pertence ao cone das árvores geradoras de $G$ se e somente se $t\left(V^{\prime}\right) \leq \lambda\left(\left|V^{\prime}\right|-1\right)$ para todo $V^{\prime} \subseteq V$ e t é não-negativo.

Demonstração. Sabemos que $t$ pertence ao cone das árvores geradoras se e somente se $t=\lambda t^{\prime}$,onde $t^{\prime}$ pertence ao fecho convexo e $\lambda \geq 0$. Pelo teorema 7.19, tal pertinência de $\lambda t$ ocorre se e somente se

$$
\begin{aligned}
t(V) & =\lambda(|V|-1) ; & & \\
t\left(V^{\prime}\right) & \leq \lambda\left(\left|V^{\prime}\right|-1\right) & & \text { para todo } V^{\prime} \subseteq V ; \\
t(e) & \geq \lambda(0) & & \text { para todo } e \in E .
\end{aligned}
$$

Temos então caracterizações para o cone e o reticulado das árvores geradoras. Como foi feito para os circuitos, vamos considerar a seguir as relações entre esses conjuntos gerados e o cone inteiro.

\section{Cone inteiro das árvores geradoras}

Quando consideramos o cone inteiro das árvores geradoras, estamos considerando combinações inteiras não-negativas de vetores inteiros não-negativos. Portanto, se estamos interessados em obter condições necessárias e suficientes para que um vetor $t$ pertença ao cone inteiro das árvores geradoras, podemos supor sem perda de generalidade que $t$ atribui custos inteiros não-negativos a $E$.

Custos inteiros não-negativos são particularmente interessantes porque podem ser interpretados como a quantidade de arestas paralelas entre dois vértices. Seja $G^{\prime}=\left(V^{\prime}, E^{\prime}\right)$ um grafo tal que $V=V^{\prime}$ e, para quaisquer $u, v \in V^{\prime}$ tais que $u \neq v$, o número de cópias de $\{u, v\}$ em $E^{\prime}$ é a soma dos custos de todas as cópias de $\{u, v\}$ em $E$. A partir dessa definição, podemos verificar a seguinte equivalência:

o vetor $t$ pertence ao cone inteiro das árvores geradoras do grafo $G$ se e somente se o conjunto de arestas do grafo $G^{\prime}$ pode ser particionado em árvores geradoras desse grafo. 

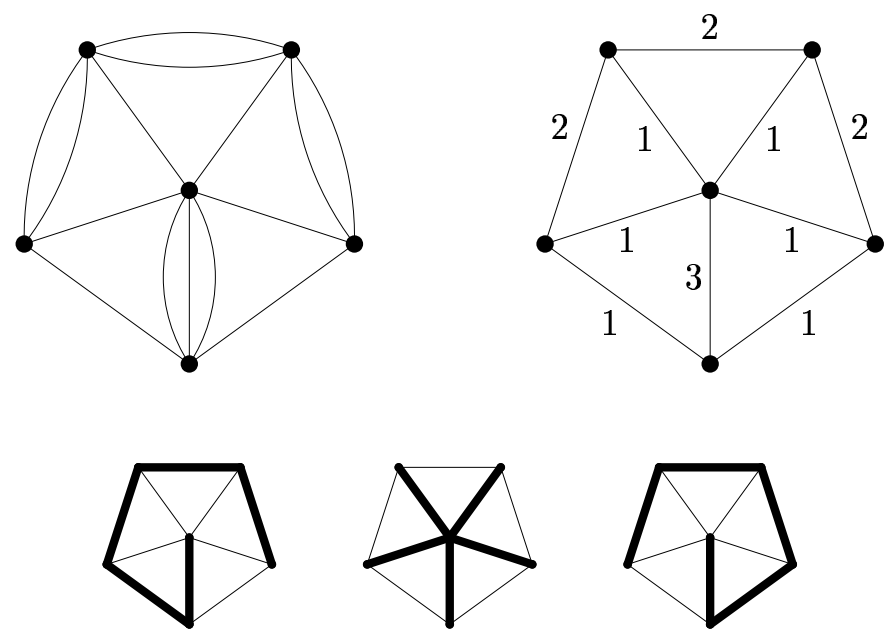

Figura 7.22: Acima à esquerda, um exemplo de grafo com arestas paralelas. Acima à direita, a transformação dessas arestas paralelas em custos inteiros não-negativos. Abaixo, árvores geradoras do grafo cuja soma gera esses custos.

A figura 7.22 mostra um exemplo dessa equivalência. Podemos utilizar essa transformação de custos em arestas paralelas devido ao fato de que árvores geradoras não podem conter arestas paralelas, visto que tais arestas formam um circuito.

Pelo mesmo motivo, não podemos obter uma equivalência análoga quando consideramos conjuntos gerados por circuitos. De fato, podemos verificar a partir da figura 7.23 que o contra-exemplo apresentado no final da seção anterior deixa de ser um contra-exemplo após a transformação.
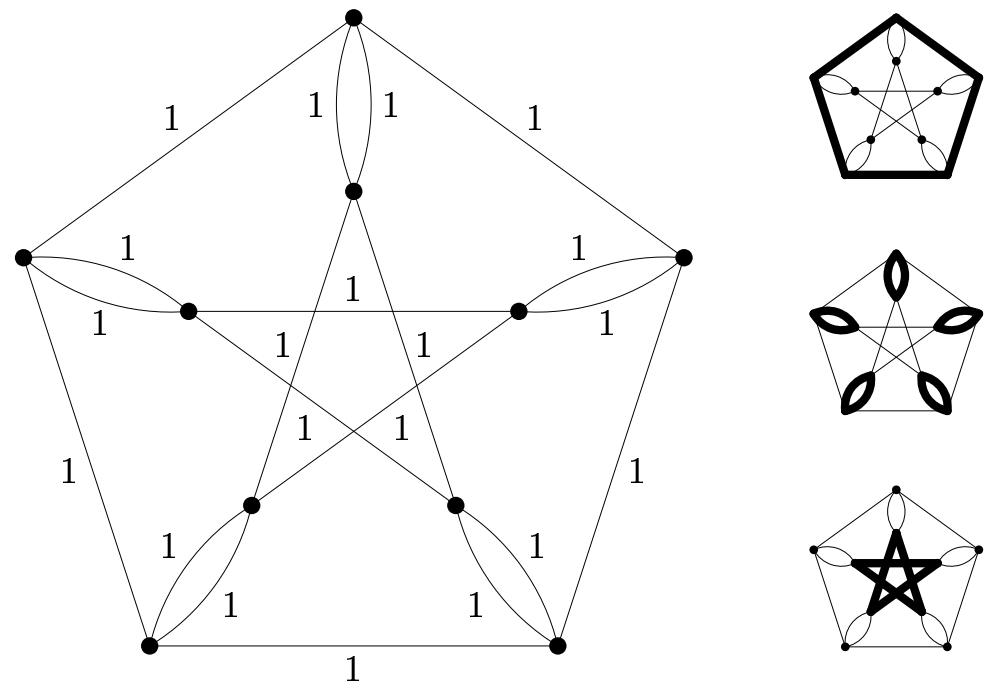

Figura 7.23: Exemplo de uma função de custos que pertence ao cone inteiro dos circuitos. Neste caso em particular, a função é gerada pela soma de sete circuitos.

Estamos, portanto, interessados em obter uma caracterização dos grafos cujo conjunto de arestas pode ser particionado em árvores geradoras. TutTe [1961] e NASH-WiLliams [1961] demonstraram que um grafo $G=(V, E)$ admite tal partição em árvores se e somente se: 
(i) $\lambda:=|E| /(|V|-1)$ é um inteiro não-negativo;

(ii) $|\delta(\mathcal{P})| \geq \lambda(|\mathcal{P}|-1)$ para toda partição $\mathcal{P}$ de $V$.

Se $T$ é uma árvore geradora desse grafo, sabemos que $|T|=|V|-1$ e também podemos verificar que $|T \cap \delta(\mathcal{P})| \leq|\mathcal{P}|-1$ para toda partição $\mathcal{P}$ de $V$. Logo, podemos concluir que as condições (i) e (ii) são necessárias. Portanto, podemos obter a caracterização descrita se demonstrarmos que

se $\lambda:=|E| /(|V|-1)$ é um inteiro não-negativo, então existem $\lambda$ árvores geradoras mutuamente disjuntas em $G$ ou existe uma partição $\mathcal{P}$ de $V$ tal que $|\delta(\mathcal{P})|<\lambda(|\mathcal{P}|-1)$.

Vamos então supor que $\lambda$ é um inteiro não-negativo e descrever um algoritmo que foi apresentado originalmente por EDMONDS [1965b]. Esse algoritmo consiste em uma seqüência de iterações tal que, em cada iteração, temos florestas mutuamente disjuntas $F_{1}, \ldots, F_{\lambda}$ em $G$. Na primeira iteração, definimos $F_{i}:=\emptyset$ para todo $i$. Em cada iteração, ocorre uma dentre as seguintes possibilidades:

- existem florestas mutuamente disjuntas $F_{1}^{\prime}, \ldots, F_{\lambda}^{\prime}$ em $G$ tais que $\sum_{i=1}^{\lambda}\left|F_{i}^{\prime}\right|>\sum_{i=1}^{\lambda}\left|F_{i}\right|$;

- as florestas $F_{1}, \ldots, F_{\lambda}$ são árvores geradoras mutuamente disjuntas em $G$;

- existe uma partição $\mathcal{P}$ de $V$ tal que $|\delta(\mathcal{P})|<\lambda(|\mathcal{P}|-1)$.

Se a primeira possibilidade ocorre, substituímos $F_{1}, \ldots, F_{\lambda}$ por $F_{1}^{\prime}, \ldots, F_{\lambda}^{\prime}$ e começamos uma nova iteração. Como a soma $\sum_{i=1}^{\lambda}\left|F_{i}\right|$ é limitada superiormente por $|E|$, uma das duas outras possibilidades ocorre após um número finito de iterações.

Para garantir que uma das possibilidades ocorra, o algoritmo considera o grafo auxiliar obtido a partir de $F_{1}, \ldots, F_{\lambda}$. Tal grafo é um grafo dirigido $D=(N, A)$ definido da seguinte maneira:

(0) $N=E \cup\{u, v\}$, onde $u, v \notin E$ e $u \neq v$;

(1) se $e \in E \backslash \bigcup_{i=1}^{\lambda} F_{i}$, então $(u, e)$ é um arco de $D$;

(2) se existe um índice $i$ e uma aresta $e$ tais que $e \notin F_{i}$

e $F_{i} \cup\{e\}$ é uma floresta, então $(e, v)$ é um arco de $D$;

(3) se existe um índice $i$ e arestas $e, f$ tais que $e \notin F_{i}, f \in F_{i}$

e $\left(F_{i} \cup\{e\}\right) \backslash\{f\}$ é uma floresta, então $(e, f)$ é um arco de $D$.

Dizemos que o vértice $u$ é a fonte de $D$ e que o vértice $v$ é o sorvedouro de $D$. Em relação às definições (2) e (3), dizemos que a floresta $F_{i}$ é correspondente ao respectivo arco. A figura 7.24 mostra um exemplo simples de grafo auxiliar definido por duas florestas.

Vamos demonstrar que, se existe um $(u, v)$-caminho em $D$, então existem florestas mutuamente disjuntas $F_{1}^{\prime}, \ldots, F_{\lambda}^{\prime}$ em $G$ tais que $\sum_{i=1}^{\lambda}\left|F_{i}^{\prime}\right|>\sum_{i=1}^{\lambda}\left|F_{i}\right|$. Mais precisamente, vamos descrever como podemos obter tais florestas a partir de um $(u, v)$-caminho de comprimento minimo.

Considere um $(u, v)$-caminho mínimo em $D$ e sejam $\left(e_{1}, f_{1}\right), \ldots\left(e_{l}, f_{l}\right)$ os arcos de tipo (3) desse caminho cuja floresta correspondente é $F_{i}$. Seja $F_{i}^{\prime}$ um conjunto definido da seguinte maneira:

$$
F_{i}^{\prime}:=\left(F_{i} \cup\left\{e_{1}, \ldots, e_{l}\right\}\right) \backslash\left\{f_{1}, \ldots, f_{l}\right\}
$$

O lema a seguir demonstra que a minimalidade do caminho garante que esse conjunto é uma floresta. 

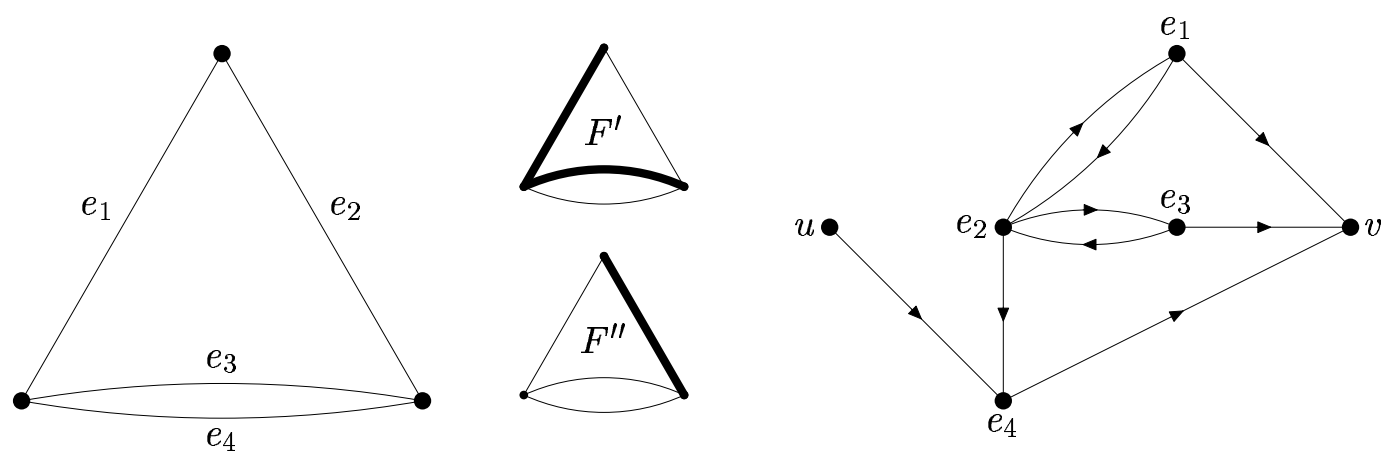

Figura 7.24: um exemplo de grafo, duas florestas disjuntas desse grafo e o grafo auxiliar associado a essas duas florestas.

Lema 7.21. Seja $G=(V, E)$ um grafo, sejam $F_{1}, \ldots, F_{\lambda}$ florestas mutuamente disjuntas desse grafo, seja $D=(N, A)$ o grafo auxiliar obtido a partir dessas florestas e sejam u, $v$ respectivamente a fonte e o sorvedouro desse grafo auxiliar. Suponha que $\left(e_{1}, f_{1}\right), \ldots,\left(e_{l}, f_{l}\right)$ são os arcos do tipo (3) correspondentes a uma floresta $F_{i}$ em um $(u, v)$-caminho de $D$. Se o comprimento desse caminho é mínimo, então $\left(F_{i} \cup\left\{e_{1}, \ldots, e_{l}\right\}\right) \backslash\left\{f_{1}, \ldots, f_{l}\right\}$ é uma floresta.

Demonstração. Suponha que o conjunto $\left(F_{i} \cup\left\{e_{1}, \ldots, e_{l}\right\}\right) \backslash\left\{f_{1}, \ldots, f_{l}\right\}$ contém um circuito $C$. Esse circuito contém pelo menos duas arestas dentre $e_{1}, \ldots, e_{l}$, pois sabemos que $\left(F_{i} \cup\left\{e_{j}\right\}\right) \backslash\left\{f_{j}\right\}$ é floresta para todo $j$. Sejam $e_{1}^{\prime}, \ldots, e_{r}^{\prime}$ as arestas dentre $e_{1}, \ldots, e_{l}$ que pertencem a $C$ e sejam $f_{1}^{\prime}, \ldots, f_{r}^{\prime}$ as arestas dentre $f_{1}, \ldots, f_{l}$ correspondentes. Vamos supor sem perda de generalidade que $e_{r}^{\prime}$ é o vértice cuja distância ao sorvedouro $v$ é mínima dentre $e_{1}^{\prime}, \ldots, e_{r}^{\prime}$.

Se para algum $j$ temos que $F_{i} \cup\left\{e_{j}^{\prime}\right\}$ é floresta, então $\left(e_{j}^{\prime}, v\right)$ é um arco do grafo auxiliar e, portanto, o comprimento do caminho não é mínimo. Vamos então supor que $F_{i} \cup\left\{e_{j}^{\prime}\right\}$ contém um circuito para todo $j$. Nesse caso, $F_{i} \cup\left\{e_{r}^{\prime}\right\}$ contém um circuito $C_{r}^{\prime}$ e esse circuito contém $e_{r}^{\prime}, f_{r}^{\prime}$. Por outro lado, sabemos que $f_{r}^{\prime}$ não pertence ao circuito $C$ por hipótese. Podemos então concluir que

$$
e_{r}^{\prime} \in C \cap C_{r}^{\prime} \quad \text { e } \quad f_{r}^{\prime} \notin C \cap C_{r}^{\prime} \quad \text { e } \quad f_{r}^{\prime} \in C \cup C_{r}^{\prime} .
$$

Como $\left(C \cup C_{r}^{\prime}\right) \backslash\left(C \cap C_{r}^{\prime}\right)$ é uma união de circuitos disjuntos, existe um circuito $C^{\prime \prime}$ ao qual $f_{r}^{\prime}$ pertence mas $e_{r}^{\prime}$ não pertence. Esse circuito contém pelo menos uma aresta dentre $e_{1}^{\prime}, \ldots, e_{r-1}^{\prime}$, pois caso contrário estaria contido em $F_{i}$. Se contém apenas uma dessas arestas, digamos $e_{j}^{\prime}$, então temos que $\left(F_{i} \cup\left\{e_{j}^{\prime}\right\}\right) \backslash\left\{f_{r}^{\prime}\right\}$ é floresta. Nesse caso, $\left(e_{j}^{\prime}, f_{r}^{\prime}\right)$ é um arco do grafo auxiliar e o caminho não é mínimo. Suponha então que pelo menos duas dessas arestas pertencem a $C^{\prime \prime}$.

Para cada $e_{j}^{\prime}$ em $C^{\prime \prime} \cap\left\{e_{1}^{\prime}, \ldots, e_{r-1}^{\prime}\right\}$, o circuito $C_{j}^{\prime}$ de $F_{i} \cup\left\{e_{j}^{\prime}\right\}$ não pode ser o próprio $C^{\prime \prime}$, pois $C^{\prime \prime}$ contém outra aresta dentre $e_{1}^{\prime}, \ldots, e_{r-1}^{\prime}$ além de $e_{j}^{\prime}$. Por outro lado, $e_{j}^{\prime}$ pertence à intersecção de $C_{j}^{\prime}$ e $C^{\prime \prime}$. Temos então dois casos possíveis: se $f_{r}^{\prime}$ pertence a $C_{j}^{\prime}$, então o arco $\left(e_{j}^{\prime}, f_{r}^{\prime}\right)$ está no grafo auxiliar e o caminho não é mínimo. Senão, $f_{r}^{\prime}$ pertence a $\left(C_{j}^{\prime} \cup C^{\prime \prime}\right) \backslash\left(C_{j}^{\prime} \cap C^{\prime \prime}\right)$ e, portanto, podemos obter um circuito que contém $f_{r}^{\prime}$ mas não contém $\left\{e_{r}^{\prime}, e_{j}^{\prime}\right\}$. Repetindo o argumento, eventualmente nos restringimos ao caso em que temos um circuito que contém $f_{r}^{\prime}$ mas não contém $r-1$ arestas dentre $e_{1}^{\prime}, \ldots, e_{r}^{\prime}$. Nesse caso, um arco entre a aresta restante e $f_{r}^{\prime}$ pertence ao grafo auxiliar.

Dentre as florestas que são correspondentes a algum arco de um $(u, v)$-caminho em $D$, uma em particular é correspondente a um arco do tipo (2). O lema anterior pode ser estendido a esse caso. 
Corolário 7.22. Seja $G=(V, E)$ um grafo, sejam $F_{1}, \ldots, F_{\lambda}$ florestas mutuamente disjuntas desse grafo, seja $D=(N, A)$ o grafo auxiliar obtido a partir dessas florestas e sejam u, $v$ respectivamente a fonte e o sorvedouro desse grafo auxiliar. Suponha que $\left(e_{1}, f_{1}\right), \ldots,\left(e_{l}, f_{l}\right)$ são os arcos do tipo (3) correspondentes a uma floresta $F_{i}$ em um $(u, v)$-caminho de D. Seja $(e, v)$ o arco desse caminho que entra em $v$ e suponha que $F_{i}$ é correspondente a esse arco. Se o comprimento desse caminho é minimo, então $\left(F_{i} \cup\left\{e_{1}, \ldots, e_{l}, e\right\}\right) \backslash\left\{f_{1}, \ldots, f_{l}\right\}$ é uma floresta.

Demonstração. Se $\left(F_{i} \cup\left\{e_{1}, \ldots, e_{l}\right\}\right) \backslash\left\{f_{1}, \ldots, f_{l}\right\}$, não é uma floresta, então sabemos pelo lema 7.21 que o comprimento do caminho não é mínimo. Vamos supor então que esse conjunto é uma floresta. Nesse caso, se $\left(F_{i} \cup\left\{e_{1}, \ldots, e_{l}, e\right\}\right) \backslash\left\{f_{1}, \ldots, f_{l}\right\}$ não é uma floresta, então contém um circuito ao qual $e$ pertence. Através de argumentos análogos aos da demonstração do lema 7.21, podemos reduzir a demonstração ao caso em que esse circuito contém uma única aresta $e_{j}$ dentre $e_{1}, \ldots, e_{l}$. Podemos concluir então que o comprimento do caminho não é mínimo, pois $F_{i} \cup\left\{e_{j}\right\}$ é floresta.

Podemos então considerar as florestas $F_{1}^{\prime}, \ldots, F_{\lambda}^{\prime}$ definidas da seguinte forma: para todo $i$,

- se $F_{i}$ não é correspondente a nenhum arco do $(u, v)$-caminho mínimo, temos $F_{i}^{\prime}:=F_{i}$

- se $F_{i}$ é correspondente apenas a arcos do tipo (3) no $(u, v)$-caminho mínimo, temos $F_{i}^{\prime}:=\left(F_{i} \backslash\left\{e_{1}, \ldots, e_{l}\right\}\right) \backslash\left\{f_{1}, \ldots, f_{l}\right\}$;

- se $F_{i}$ é correspondente a arcos do tipo (3) e do tipo (2) no $(u, v)$-caminho mínimo, temos $F_{i}^{\prime}:=\left(F_{i} \backslash\left\{e_{1}, \ldots, e_{l}, e\right\}\right) \backslash\left\{f_{1}, \ldots, f_{l}\right\}$,

onde as arestas $e_{1}, \ldots, e_{l}$ são definidas como no lema 7.21 e no corolário 7.22. Podemos verificar que essas florestas satisfazem $\sum_{i=1}^{\lambda}\left|F_{i}^{\prime}\right|>\sum_{i=1}^{\lambda}\left|F_{i}\right|$, como queríamos.

Se por outro lado não existem $(u, v)$-caminhos em $D$, então o lema a seguir garante que uma das outras duas possibilidades que foram enunciadas são de fato válidas para a iteração corrente.

Lema 7.23. Seja $G=(V, E)$ um grafo, sejam $F_{1}, \ldots, F_{\lambda}$ florestas mutuamente disjuntas desse grafo, seja $D=(N, A)$ o grafo auxiliar obtido a partir dessas florestas e sejam $u, v$ respectivamente a fonte $e$ o sorvedouro desse grafo auxiliar. Se não existem $(u, v)$-caminhos em $D$, então as florestas $F_{1}, \ldots, F_{\lambda}$ são árvores geradoras de $G$ ou existe uma partição $\mathcal{P}$ de $V$ tal que $|\delta(\mathcal{P})|<\lambda(|\mathcal{P}|-1)$.

Demonstração. Seja $C$ um subconjunto de $\bigcup_{i=1}^{\lambda} F_{i}$ tal que uma aresta $e$ pertence a $C$ se e somente se existe um $(u, e)$-caminho em $D$. Primeiramente vamos demonstrar que, para todo índice $i$,

$$
\left|F_{i} \cap C\right|=r(C) \text {. }
$$

Suponha por contradição que existe um índice $i$ e uma aresta $e \in C$ tais que $e \notin F_{i}$ e $\left(F_{i} \cap C\right) \cup\{e\}$ é uma floresta. Sabemos que $F_{i} \cup\{e\}$ não pode ser uma floresta, pois caso contrário $(e, v)$ seria um arco de $D$ e, portanto, existiria um $(u, v)$-caminho em $D$. Logo, existe um circuito em $F_{i} \cup\{e\}$. Ademais, como $\left(F_{i} \cap C\right) \cup\{e\}$ é uma floresta, existe uma aresta $f$ nesse circuito tal que $f \in C$. Como sabemos que $\left(F_{i} \cup\{e\}\right) \backslash\{f\}$ é uma floresta, temos que $(e, f)$ é um arco de $D$. Mas isso é uma contradição, pois implica que $f$ pertence a $C$.

Sejam $\left(V_{1}, E_{1}\right), \ldots,\left(V_{k}, E_{k}\right)$ as componentes de $(V, C)$ e seja $\mathcal{P}:=\left\{V_{1}, \ldots, V_{k}\right\}$. Sabemos que, para quaisquer $i, j$, o conjunto $F_{i} \cap E_{j}$ é árvore geradora de $\left(V_{j}, E_{j}\right)$, pois $\left|F_{i} \cap C\right|=r(C)$. Logo,

$$
\left|\delta(\mathcal{P}) \cap \bigcup_{i=1}^{\lambda} F_{i}\right| \leq \lambda(|\mathcal{P}|-1) .
$$


Vamos demonstrar que $\delta(\mathcal{P}) \subseteq \bigcup_{i=1}^{\lambda} F_{i}$ para podermos concluir que $|\delta(\mathcal{P})| \leq \lambda(|\mathcal{P}|-1)$. Suponha por contradição que uma aresta $e$ pertence a $\delta(\mathcal{P})$ mas não pertence a nenhuma das florestas. Seja $i$ um índice qualquer. Sabemos que $F_{i} \cup\{e\}$ não é uma floresta, pois caso contrário existiria um $(u, v)$-caminho em $D$. Logo, podemos verificar que existe uma aresta $f \neq e$ em $F_{i} \cap \delta(\mathcal{P})$ tal que $\left(F_{i} \cup\{e\}\right) \backslash\{f\}$ é uma floresta. Portanto, existe um $(u, f)$-caminho em $D$. Mas isso é uma contradição, pois pela definição de $\mathcal{P}$ podemos verificar que $\delta(\mathcal{P}) \cap C=\emptyset$.

Temos então que $|\delta(\mathcal{P})|=\lambda(|\mathcal{P}|-1)$ ou $|\delta(\mathcal{P})|<\lambda(|\mathcal{P}|-1)$. No primeiro caso, sabemos pela definição de $\mathcal{P}$ que as florestas $F_{1}, \ldots, F_{\lambda}$ são árvores geradoras do grafo $G$, como queríamos.

Assim, a correção do algoritmo descrito demonstra o teorema de Tutte e Nash-Williams.

Teorema 7.24. Seja $G=(V, E)$ um grafo conexo e seja $\lambda:=(|V|-1) /|E|$. As arestas de $G$ podem ser particionadas em árvores geradoras de $G$ se e somente se $\lambda$ é um inteiro e $|\delta(\mathcal{P})| \geq \lambda(|\mathcal{P}|-1)$ para toda partição $\mathcal{P}$ de $V$.

A partir da equivalência descrita no início desta seção, o teorema pode ser reformulado como:

Teorema 7.25. Seja $G=(V, E)$ um grafo conexo, seja $t$ um vetor indexado pelas arestas desse grafo e seja $\lambda:=t(E) /(|V|-1)$. O vetor $t$ pertence ao cone inteiro das árvores geradoras de $G$ se $e$ somente se $t$ é inteiro e não-negativo, $\lambda$ é um inteiro não-negativo e $t(\delta(\mathcal{P})) \geq \lambda(|\mathcal{P}|-1)$ para toda partição $\mathcal{P}$ de $V$.

Informalmente, a desigualdade $t(\delta(\mathcal{P})) \geq \lambda(|\mathcal{P}|-1)$ pode ser interpretada como atribuição de muito custo às arestas que não estão contidas nas partes de $\mathcal{P}$.

Vamos então considerar uma versão complementar dessa descrição: atribuição de pouco custo às arestas que estão contidas nas partes de $\mathcal{P}$. Essa reinterpretação é particularmente interessante porque estabelece uma relação com a caracterização do cone das árvores geradoras. Demonstramos na seção anterior que, esse cone contém precisamente vetores que atribuem uma quantidade limitada de custo às arestas contidas em um conjunto de vértices.

Formalmente, a relação entre as duas descrições pode ser verificada através de uma manipulação algébrica simples. Se $t\left(V^{\prime}\right) \leq \lambda\left(\left|V^{\prime}\right|-1\right)$ para todo $V^{\prime} \subseteq V$ e $\mathcal{P}$ é uma partição de $V$, temos que

$$
\begin{aligned}
t(\delta(\mathcal{P})) & =t(V)-\sum_{V^{\prime} \in \mathcal{P}} t\left(V^{\prime}\right) \\
& \leq \lambda(|V|-1)-\sum_{V^{\prime} \in \mathcal{P}} \lambda\left(\left|V^{\prime}\right|-1\right) \\
& =\lambda|V|-\lambda-\lambda \sum_{V^{\prime} \in \mathcal{P}}\left|V^{\prime}\right|+\lambda \sum_{V^{\prime} \in \mathcal{P}} 1 \\
& =\lambda|V|-\lambda-\lambda|V|+\lambda|\mathcal{P}| \\
& =\lambda(|\mathcal{P}|-1) .
\end{aligned}
$$

Podemos concluir então que as duas condições do teorema são válidas quando o vetor pertence ao cone e ao reticulado das árvores geradoras do grafo. Temos então o resultado mais importante desta seção e, no contexto deste trabalho, a principal diferença entre árvores geradoras e circuitos:

Teorema 7.26. Os vetores de incidência das árvores geradoras de um grafo conexo formam uma base de Hilbert. 
Demonstração. Seja $G=(V, E)$ um grafo conexo, seja $t$ um vetor indexado pelas arestas desse grafo e seja $\lambda:=t(E) /(|V|-1)$. Suponha que $t$ pertence ao reticulado e ao cone das árvores geradoras de $G$. Pelo teorema 7.18, sabemos que $t$ é um vetor inteiro e $\lambda$ é um inteiro. Pelo teorema 7.20, sabemos que $t$ é um vetor não-negativo e $t\left(V^{\prime}\right) \leq \lambda\left(\left|V^{\prime}\right|-1\right)$ para todo $V^{\prime} \subseteq V$. Dessa propriedade segue que $\lambda$ é não-negativo e $t(\delta(\mathcal{P})) \geq \lambda(|\mathcal{P}|-1)$ para toda partição $\mathcal{P}$ de $V$. Portanto, pelo teorema 7.25, podemos concluir que $t$ pertence ao cone inteiro das árvores geradoras de $G$.

Temos então que os vetores de incidência das árvores geradoras de $G$ formam um sistema gerador de Hilbert. Ademais, como uma árvore geradora contém exatamente $|V|-1$ arestas, podemos verificar que nenhum desses vetores é gerado por uma combinação inteira não-negativa dos outros. Logo, podemos concluir por definição que tais vetores de incidência formam uma base de Hilbert.

Na subseção a seguir, vamos generalizar esse teorema para florestas maximais de grafo não necessariamente conexos e considerar limitantes para o posto de Carathéodory dessas florestas.

\section{Posto de Carathéodory das florestas maximais}

Sejam $\left(V_{1}, E_{1}\right), \ldots,\left(V_{k}, E_{k}\right)$ as componentes do grafo $G$. Pela definição de floresta, sabemos que um conjunto $F \subseteq E$ é uma floresta maximal de $G$ se e somente se existem $T_{1}, \ldots, T_{k} \subseteq E$ tais que $T_{i}$ é uma árvore geradora de $\left(V_{i}, E_{i}\right)$ para todo $i$ e $F:=\bigcup_{i=1}^{k} T_{i}$.

Através dessa relação entre florestas maximais e árvores geradoras de subgrafos, podemos verificar que todas as caracterizações para conjuntos gerados por árvores geradoras podem ser generalizadas naturalmente para florestas maximais. Podemos obter, por exemplo, uma caracterização do cone:

Teorema 7.27. Seja $G=(V, E)$ um grafo, seja $t$ um vetor indexado pelas arestas desse grafo $e$ seja $\lambda:=t(E) / r(E)$. O vetor $t$ pertence ao cone das árvores geradoras de $G$ se e somente se $t$ é não-negativo e $t\left(E^{\prime}\right) \leq \lambda r\left(E^{\prime}\right)$ para todo $E^{\prime} \subseteq E$.

Como corolário da generalização, podemos estender para florestas maximais o teorema principal:

Teorema 7.28. Os vetores de incidência das florestas maximais de um grafo formam uma base de Hilbert.

Para encerrar esta seção, vamos considerar limitantes para o posto de Carathéodory do conjunto dos vetores de incidência das florestas maximais. A partir do corolário 6.2, podemos verificar que:

Teorema 7.29. Seja $G=(V, E)$ um grafo e seja $t$ um vetor indexado pelas arestas desse grafo. Se $t$ pertence ao cone inteiro dos vetores de incidência das florestas maximais de $G$, então $t$ é gerado por uma combinação inteira não-negativa de não mais do que $2|E|-1$ desses vetores.

Se as conjecturas que apresentamos no capítulo 6 são válidas para essa base de Hilbert específica, então esse limitante pode ser melhorado para $|E|$. Porém, ainda não se conhece uma demonstração ou um contra-exemplo para as conjecturas nesse caso.

Por outro lado, vamos demonstrar a seguir que o posto de Carathéodory das florestas maximais de $G$ é limitado superiormente por um valor melhor do que $2|E|-1$. Vamos obter o limitante

$$
|E|+r(E)-1
$$


A demonstração será apresentada de maneira bastante sucinta, pois quase todos os argumentos utilizados são análogos a argumentos de demonstrações anteriores. Em particular, vamos considerar uma indução similar à que descrevemos para caracterizar o cone dos circuitos.

Seja $b$ um vetor que pertence ao cone inteiro das florestas maximais de $G$ e sejam $b_{1}, \ldots, b_{m}$ os vetores de incidência dessas florestas maximais. Para todo $i$, seja $\lambda_{b, i}^{*}$ definido da seguinte maneira:

$$
\lambda_{b, i}^{*}:=\max \left\{\lambda: b-\lambda b_{i} \text { pertence ao cone das florestas maximais }\right\} .
$$

Se para algum $i$ temos que $\lambda_{b, i}^{*}$ é inteiro, então o vetor $c:=b-\lambda_{b, i}^{*} b_{i}$ também pertence ao cone inteiro das florestas maximais. Podemos então considerar uma indução em $|E|$, cuja base é o caso no qual existe apenas uma aresta no grafo. A partir do teorema 7.27, podemos verificar que:

existe e $\in E$ tal que $c(e)=0$ ou existe $E^{\prime} \subset E$ tal que $c\left(E^{\prime}\right)=\mu r\left(E^{\prime}\right)$, onde $\mu:=\lambda-1$ e $\lambda:=t(E) / r(E)$.

Se existe $e \in E$ tal que $c(e)=0$, podemos considerar a hipótese de indução sobre a restrição de $c$ ao conjunto das arestas cujo custo atribuído por $c$ é não-nulo. Dessa hipótese segue que tal restrição é gerada por uma combinação inteira não-negativa de não mais do que $|E|+r(E)-2$ florestas maximais do respectivo subgrafo. Podemos então concluir que o mesmo limitante vale para o vetor $c$ em $G$. Portanto, temos que $b$ é gerado por uma combinação inteira não-negativa de $b_{i} \mathrm{e}$ não mais do que $|E|+r(E)-2$ outros vetores de incidência de florestas maximais.

Vamos agora considerar o caso em que $c$ é positivo e $c\left(E^{\prime}\right)=r\left(E^{\prime}\right)$ para algum $E^{\prime} \subset E$. Dizemos nesse caso que $E^{\prime}$ é um conjunto $c$-justo. $\mathrm{O}$ fato de que esse subconjunto é próprio garante que:

(i) o subgrafo $\left(V, E^{\prime}\right)$ é menor do que $G$;

(ii) se $\left(V_{1}, E_{1}\right), \ldots,\left(V_{k}, E_{k}\right)$ são as componentes desse subgrafo, então o grafo obtido a partir da contração de $V_{1}, \ldots, V_{k}$, é menor do que $G$.

Seja $\left(V^{\prime \prime}, E^{\prime \prime}\right)$ o grafo obtido a partir da contração de $V_{1}, \ldots, V_{k}$, seja $c^{\prime}$ a restrição de $c$ a $E^{\prime}$ e seja $c^{\prime \prime}$ a adaptação de $c$ à contração de $V_{1}, \ldots, V_{k}$. Como os grafos $\left(V, E^{\prime}\right)$ e $\left(V^{\prime \prime}, E^{\prime \prime}\right)$ são menores do que $G$, podemos aplicar a hipótese de indução sobre os vetores $c^{\prime}$ e $c^{\prime \prime}$, de modo a concluir que:

- o vetor $c^{\prime}$ é gerado por uma combinação inteira não-negativa de não mais do que $\left|E^{\prime}\right|+r\left(E^{\prime}\right)-1$ florestas maximais do grafo $\left(V, E^{\prime}\right)$;

- o vetor $c^{\prime \prime}$ é gerado por uma combinação inteira não-negativa de não mais do que $\left|E^{\prime \prime}\right|+r\left(E^{\prime \prime}\right)-1$ florestas maximais do grafo $\left(V^{\prime \prime}, E^{\prime \prime}\right)$.

Ademais, podemos verificar que a soma da extensão do vetor de incidência de uma floresta maximal de $\left(V, E^{\prime}\right)$ com a expansão do vetor de incidência de uma floresta maximal de $\left(V^{\prime \prime}, E^{\prime \prime}\right)$ gera o vetor de incidência de uma floresta maximal de $G$. A figura 7.25 mostra um exemplo.

Portanto, utilizando uma estratégia de alinhamento sobre as duas combinações, podemos obter uma combinação inteira não-negativa de florestas maximais de $G$ que gera o vetor $c$. Também podemos verificar que, como conseqüência do alinhamento, tal combinação é de não mais do que

$$
\left(\left|E^{\prime}\right|+r\left(E^{\prime}\right)-1\right)+\left(\left|E^{\prime \prime}\right|+r\left(E^{\prime \prime}\right)-1\right)-1
$$

florestas maximais. Podemos então concluir que $b$ é gerado por uma combinação inteira não-negativa de não mais do que $|E|+r(E)-2$ florestas maximais, um limitante melhor do que queríamos. 

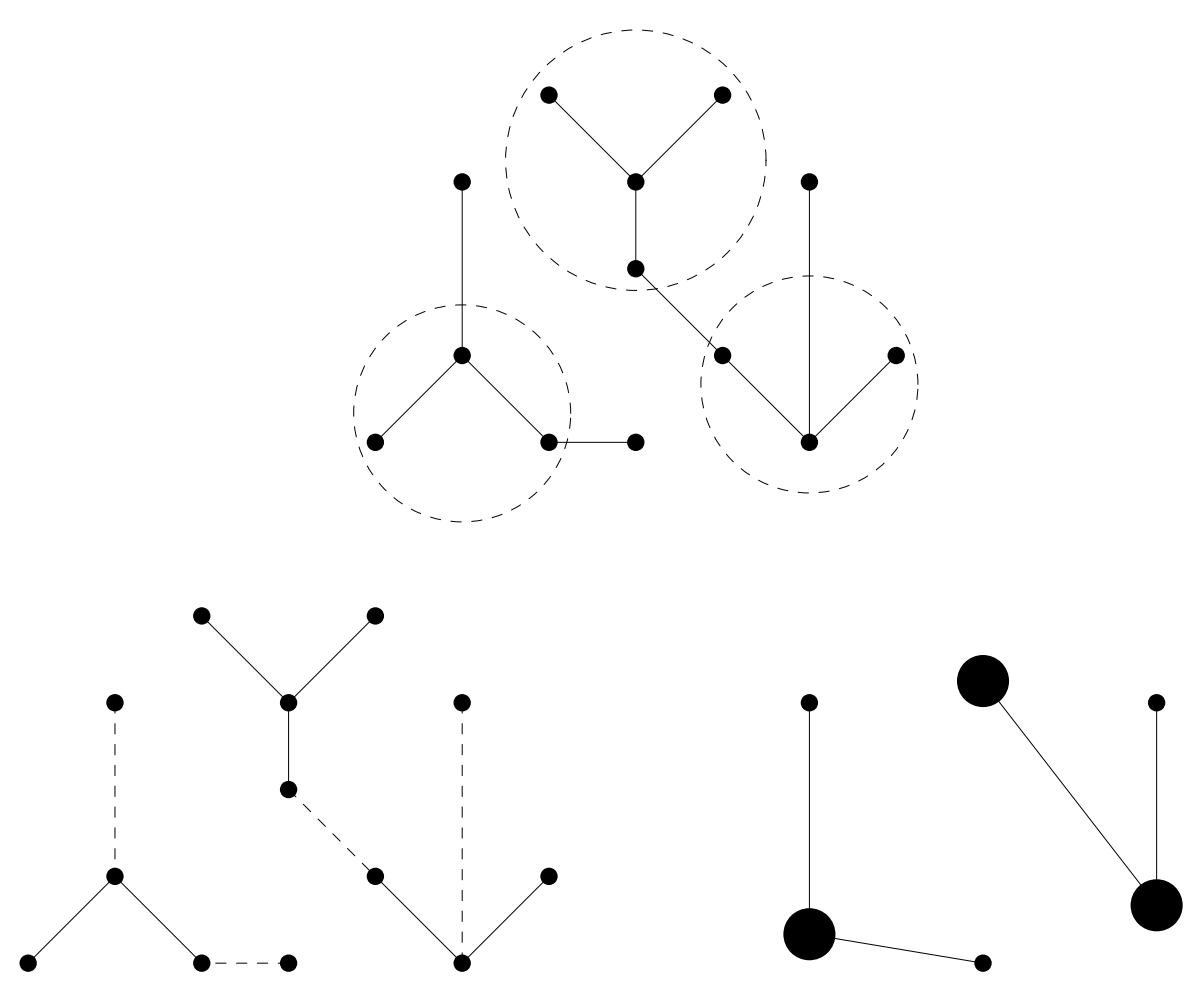

Figura 7.25: Acima, um exemplo de floresta geradora e três subconjuntos de vértices indicados pelos círculos. Abaixo à esquerda, o subgrafo induzido por esses subconjuntos de vértices. Abaixo à direita, o grafo obtido a partir da contração dos três conjuntos.

Vamos agora supor que $\lambda_{i}^{*}$ não é inteiro. Nesse caso, vamos considerar $c:=b-\left\lfloor\lambda_{b, i}^{*}\right\rfloor b_{i}$ ao invés de $b-\lambda_{b, i}^{*}$ para ainda podermos afirmar que $c$ pertence ao cone inteiro das florestas maximais. Porém, ao considerarmos esse coeficiente $\left\lfloor\lambda_{b, i}^{*}\right\rfloor$, não podemos mais garantir que $c(e)=0$ para algum $e \in E$ ou $c\left(E^{\prime}\right)<r\left(E^{\prime}\right)$ para algum $E^{\prime} \subset E$, como ocorre para $\lambda_{c, i}^{*}$.

No entanto, podemos utilizar o lema a seguir para considerar uma segunda estratégia de redução:

Lema 7.30. Seja $G=(V, E)$ um grafo, sejam $b_{1}, \ldots, b_{m}$ os vetores de incidência das florestas maximais desse grafo, seja $b$ um vetor indexado pelas arestas de $G$, seja $i$ um indice tal que $\lambda_{b, i}^{*}$ não é inteiro e seja $c:=b-\left\lfloor\lambda_{b, i}^{*}\right\rfloor b_{i}$. Se b é positivo, b pertence ao cone inteiro de $b_{1}, \ldots, b_{m}$ e nãa existem subconjuntos b-justos de $E$, então existem $j$ e $E^{\prime} \subseteq E$ tais que $E^{\prime}$ é $\left(c-b_{j}\right)$-justo.

Demonstração. A partir das hipóteses sobre $b$ e $\lambda_{b, i}^{*}$, podemos verificar que $c$ também é positivo, $c$ também pertence ao cone inteiro de $b_{1}, \ldots, b_{m}$ e também não existem subconjuntos $c$-justos de $E$. Portanto, podemos supor sem perda de generalidade que $0<\lambda_{b, i}^{*}<1$ e que $c=b$.

Ademais, sabemos que existe $j$ tal que $c-b_{j}$ pertence ao cone inteiro de $b_{1}, \ldots, b_{m}$. Considere

$$
\left\{e_{1}, \ldots, e_{l}\right\}:=B_{i} \backslash B_{j} \quad \text { e } \quad\left\{f_{1}, \ldots, f_{l}\right\}:=B_{j} \backslash B_{i} .
$$

Considere também, para todo $r$ tal que $0 \leq r \leq l$, o conjunto $B_{r}^{\prime} \subseteq E$ definido da seguinte maneira:

$$
B_{r}^{\prime}:=\left(B_{i} \backslash\left\{e_{1}, \ldots, e_{r}\right\}\right) \cup\left\{f_{1}, \ldots, f_{r}\right\}
$$


Podemos supor sem perda de generalidade que $B_{0}^{\prime}, \ldots, B_{l}^{\prime}$ são florestas maximais. Sejam $b_{0}^{\prime}, \ldots, b_{l}^{\prime}$ os respectivos vetores de incidência dessas florestas. Considere um análogo de $\lambda_{b, i}^{*}$ para esses vetores:

$$
\mu_{b, i}^{*}:=\max \left\{\mu: b-\mu b_{i}^{\prime} \text { pertence ao cone das florestas maximais }\right\} .
$$

Por definição, temos $\mu_{b, 0}^{*}=\lambda_{b, i}^{*}$ e $\mu_{b, l}^{*}=\lambda_{b, j}^{*}$. Sabemos também que $\lambda_{b, i}^{*}<1$ e $\lambda_{b, j}^{*} \geq 1$. Portanto, podemos concluir que existe $r>0$ tal que $\mu_{b, r-1}^{*}<1$ e $\mu_{b, r}^{*} \geq 1$.

Temos então que $b-b_{r-1}^{\prime}$ não pertence ao cone das florestas maximais. Como $b$ é inteiro e positivo, não existe $e \in E$ tal que $b(e)-b_{r-1}^{\prime}(e)<0$. Logo, pelo teorema 7.27 , existe $E^{\prime} \subseteq E$ tal que

$$
b\left(E^{\prime}\right)-b_{r-1}^{\prime}\left(E^{\prime}\right)>\lambda r\left(E^{\prime}\right) \text {, onde } \lambda:=b(E) / r(e) .
$$

Sejam $x, y$ os respectivos vetores de incidência de $\left\{e_{r}\right\},\left\{f_{r}\right\}$. Como $b_{r}^{\prime}=b_{r-1}^{\prime}-x+y$, temos que

$$
\begin{aligned}
\lambda r\left(E^{\prime}\right) & \geq b\left(E^{\prime}\right)-b_{r}^{\prime}\left(E^{\prime}\right) \\
& =b\left(E^{\prime}\right)-\left(b_{r-1}^{\prime}\left(E^{\prime}\right)-x\left(E^{\prime}\right)+y\left(E^{\prime}\right)\right) \\
& \geq b\left(E^{\prime}\right)-\left(\lambda r\left(E^{\prime}\right)+1-b\left(E^{\prime}\right)\right)-x\left(E^{\prime}\right)+y\left(E^{\prime}\right) \\
& =\lambda r\left(E^{\prime}\right)+1-x\left(E^{\prime}\right)+y\left(E^{\prime}\right) .
\end{aligned}
$$

Logo, temos que $x\left(E^{\prime}\right)-y\left(E^{\prime}\right) \geq 1$ e podemos concluir que $e \in E^{\prime}$ e $f \notin E^{\prime}$. Temos então que

$$
\lambda r\left(E^{\prime}\right) \geq b\left(E^{\prime}\right)-b_{r}^{\prime}\left(E^{\prime}\right) \geq b\left(E^{\prime}\right)-b_{r-1}^{\prime}\left(E^{\prime}\right)-1 \geq \lambda r\left(E^{\prime}\right)
$$

e, portanto, que $b\left(E^{\prime}\right)-b_{r}^{\prime}\left(E^{\prime}\right)=\lambda r\left(E^{\prime}\right)$. Segue que $\mu_{r}^{*}=1$ e que o conjunto $E^{\prime}$ é $\left(b-b_{r}^{\prime}\right)$-justo.

Segue diretamente desse lema e dos argumentos anteriores que, se $\lambda_{i}^{*}$ não é inteiro, então existe $j$ tal que $b$ é uma combinação inteira não-negativa de $b_{i}, b_{j}$ e não mais do que outros $|E|-r(E)-3$ vetores de incidência de florestas maximais de $G$. Podemos obter então o limitante desejado:

Teorema 7.31. Seja $G=(V, E)$ um grafo e seja $t$ um vetor indexado pelas arestas desse grafo. Se $t$ pertence ao cone inteiro dos vetores de incidência das florestas maximais de $G$, então $t$ é gerado por uma combinação inteira não-negativa de não mais do que $|E|+r(E)-1$ desses vetores.

A demonstração que descrevemos nesta subseção foi apresentada por DE PINA E SoAREs [2003]. Como $r(E) \leq|E|$ independentemente do grafo considerado, podemos verificar que $|E|+r(E)-1$ quase sempre é um limitante melhor do que $2|E|-1$. A única exceção ocorre quando $r(E)=|E|$. Mas nesse caso o grafo tem uma única floresta maximal e, portanto, não é um grafo muito interessante. 


\section{Conclusão}

Neste trabalho, apresentamos resultados sobre bases de Hilbert e versões inteiras do teorema de Carathéodory. Ambos os tópicos são bastante ricos e muitos outros resultados relacionados aos que vimos podem ser encontrados na literatura. Em particular, há diversos exemplos combinatórios de bases de Hilbert além dos que foram exibidos no último capítulo.

Vamos listar a seguir alguns tópicos que não foram estudados mas estão relacionados de alguma forma ao assunto deste trabalho. Enfatizaremos algumas bases de Hilbert combinatórias específicas.

\section{Redução de bases}

Um dos tópicos mais importantes em teoria dos reticulados é o algoritmo para redução de bases de Lenstra, Lenstra e LovÁsz [1982], que permite a resolução em tempo polinomial de problemas de aproximação diofantina. Redução de bases é um dos ingredientes do algoritmo de LENSTRA [1983] para programação inteira em dimensão fixa que citamos no capítulo 5.

O livro de GRÖTsCHEL, Lovász E SCHRIJVER [1993] mostra como esse algoritmo e o método das elipsóides de KHACHIYAN [1979] podem ser utilizados para resolver certos problemas de otimização combinatória em tempo polinomial, através da conhecida equivalência entre separação e otimização.

\section{Total dual integralidade}

Como foi mencionado na introdução do capítulo 5, a abordagem de sistemas totalmente dual integrais neste trabalho restringiu-se às relações desses sistemas com bases de Hilbert. O livro de SchriJver [1986] apresenta uma abordagem bem mais completa, que inclui o comportamento de total dual integralidade sob certas operações, o importante conceito de total dual integralidade em caixa e caracterizações para sistemas totalmente dual integrais.

Também existem muitas outras relações min-max obtidas a partir de sistemas totalmente dual integrais além das que vimos no capítulo 7. Muitos exemplos dessas relações e seus respectivos sistemas totalmente dual integrais podem ser encontrados em outro livro de SchriJVER [2003].

\section{Famílias livres de cruzamento}

O artigo de Edmonds E GiLes [1977], citado várias vezes neste trabalho, mostra como famílias livres de cruzamento unificam demonstrações de alguns teoremas clássicos sobre matróides e relações min-max como o teorema de LuCChesi E Younger [1978].

Um arcabouço para esse tipo de demonstração é descrito em um artigo de ScHrIJVER [1984]. 


\section{Estado das conjecturas}

Dentre os trabalhos que descrevem o estado atual das conjecturas de Sebó, podemos citar os artigos de Liu [1991], Firla e Ziegler [1999] e Eisenbrand e Shmonin [2006].

A tese de doutorado de Chaourar [1993] descreve outras classes especiais de bases de Hilbert.

\section{Exemplos combinatórios}

No próprio artigo de SEBÕ [1990] são citados alguns exemplos de bases de Hilbert combinatórias que não foram abordados neste trabalho. Em particular, o autor menciona $T$-junções e fluxos, além de demonstrar um limitante para o posto de Carathéodory de arborescências que é bastante similar ao limitante para florestas maximais que vimos no capítulo 7.

Cunningham e Marsh [1978] demonstraram que o posto de Carathéodory é baixo em sistemas que descrevem o poliedro dos emparelhamentos de um grafo. Resultados similares relacionados à matriz de incidência de um grafo misto série-paralelo foram obtidos por LEE E WAKABAYASHI [2002].

\section{Emparelhamentos perfeitos}

Existe uma teoria muito rica relacionada aos conjuntos gerados pelos emparelhamentos perfeitos de um grafo. Resultados a respeito do fecho convexo e do cone foram obtidos por EDmonds [1965a] e o fecho linear é abordado em artigos de NADDEF [1982] e NADDEF E PulleyblanK [1982] e também por Edmonds, Lovász e Pulleyblank [1982] e LovÁsz [1983]. Vários resultados relacionados podem ser encontrados no livro de LovÁsz E Plummer [1986].

O reticulado dos emparelhamentos perfeitos foi caracterizado por LovÁsz [1987] e um extenso trabalho a respeito da teoria presente nessa caracterização foi feito por MURTY [1994]. Na tese de doutorado de CARVALHO [1996], demonstrou-se que para grafos matching covered existe uma base do reticulado formada apenas por vetores de incidência de emparelhamentos. Mais detalhes a respeito desse caso particular de grafos foram publicados por CaRVAlho, LuCChesi E MuRTy [1999].

\section{Considerações finais}

Juntando os resultados sobre circuitos e florestas maximais que vimos no capítulo 7 e esses resultados sobre emparelhamentos, podemos apresentar uma tabela inspirada na de MURTY [1994]:

\begin{tabular}{l|l|l|l} 
& circuitos & florestas maximais & emparelhamentos perfeitos \\
\hline fecho convexo & provavelmente difícil & caracterização completa & caracterização completa \\
\hline fecho linear & caracterização completa & caracterização completa & caracterização completa \\
\hline cone & caracterização completa & caracterização completa & caracterização completa \\
\hline reticulado & caracterização completa & caracterização completa & caracterização completa \\
\hline cone inteiro & caracterização parcial & caracterização completa & provavelmente difícil
\end{tabular}

Todos os resultados sobre florestas maximais podem ser generalizados para quaisquer matróides. O artigo de GodDyn [1993] apresenta uma abordagem extensa a respeito de conjuntos gerados por emparelhamentos perfeitos e circuitos de matróides.

Por fim, no artigo de Aardal, Weismantel e Wolsey [2002], bases de Hilbert e bases de Gröbner são utilizadas como ferramentas para a resolução de um certo tipo de programa inteiro. 


\section{Bibliografia}

[2002] K. Aardal, R. Weismantel E L. A. Wolsey, Non-standard approaches to integer programming, Discrete Applied Mathematics, volume 123 (2002), páginas 5-74. [132]

[1994] B. Alspach, L. Goddyn E C. Q. Zhang, Graphs with the circuit cover property, Transactions of the American Mathematical Society, volume 344 (1994), páginas 131-154. [115]

[1999] W. Bruns E J. Gubeladze, Normality and covering properties of affine semigroups, Journal für die Reine und Angewandte Mathematik, volume 510 (1999), páginas 161-178. [86]

[1999] W. Bruns, J. Gubeladze, M. Henk, A. Martin e R. Weismantel, A counterexample to an integer analogue of Carathéodory's theorem, Journal für die Reine und Angewandte Mathematik, volume 510 (1999), páginas 179-185. [86]

[1911] C. CARAThÉodory, Über den Variabilitätsbereich der Fourierschen Konstanten von positiven harmonischen Funktionen, Rendiconto del Circolo Matematico di Palermo, volume 32 (1911), páginas 193-217. [25]

[1996] M. H. Carvalho, Decomposição ótima em orelhas para grafos matching covered, tese de doutorado, Instituto de Computação, Campinas, 1996. [132]

[1999] M. H. Carvalho, C. L. Lucchesi e U. S. R. MurTy, Ear decompositions of matching covered graphs, Combinatorica: an International Journal on Combinatorics and the Theory of Computing, volume 19 (1999), páginas 151-174. [132]

[1981] R. Chandrasekaran, Polynomial algorithms for totally dual integral systems and extensions, em Studies on Graphs and Discrete Programming (Includes Proceedings of the Workshop on Applications of Graph Theory to Management, held at the European Institute for Advanced Studies in Management, Brussels, March 20-21, 1979, and also papers from the 10th Mathematical Programming Symposium, Montreal, August 27-31, 1979), editado por P. Hansen, volume 11 de Annals of Discrete Mathematics, North-Holland, 1981, páginas 39-51. [60]

[1993] B. ChaOURAR, Bases hilbertiennes et applications combinatoires, tese de doutorado, Institut d'Informatique et Mathématiques Appliquées de Grenoble, 1993. [132]

[1986] W. Cook, J. Fonlupt E A. Schrijver, An integer analogue of Carathéodory's theorem, Journal of Combinatorial Theory (Series B), volume 40 (1986), páginas 63-70. [72, 97]

[1984] W. Cook, L. Lovász E A. Schrijver, A polynomial-time test for total dual integrality in fixed dimension, Mathematical Programming Study, volume 22 (1984), páginas 64-69. [68] 
[1931] J. G. VAN DER CoRPut, Konstruktion der Minimalbasis für spezielle Diophantische Systeme von linear-homogenen Gleichungen und Ungleichungen, Proceedings Koninklijke Akademie van Wetenschappen te Amsterdam, volume 34 (1931), páginas 515-523. [58]

[1978] W. H. Cunningham E A. B. Marsh, A primal algorithm for optimum matching, Mathematical Programming Study, volume 8 (1978), páginas 50-72. [132]

[2005] R. Diestel, Graph Theory (Third Edition), volume 173 de Graduate Texts in Mathematics, Springer, 2005. [7]

[1983] P. D. Domich, Three new polynomially-time bounded Hermite normal form algorithms, dissertação de mestrado, School of Operations Research and Industrial Engineering, New York, 1983. [51]

[1985] P. D. Domich, R. Kannan e L. E. Trotter JR., Hermite normal form computation using modulo determinant arithmetic, relatório técnico, Center for Operations and Econometrics, Louvain-la-Neuve, 1985. [51]

[1965a] J. Edmonds, Maximum matching and a polyhedron with 0,1-vertices, Journal of Research of the National Bureau of Standards, volume 69B (1965), páginas 125-130. [132]

[1965b] J. Edmonds, Minimum partition of a matroid into independent subsets, Journal of Research of the National Bureau of Standards, volume 69B (1965), páginas 67-72. [123]

[1967] J. Edmonds, Systems of distinct representatives and linear algebra, Journal of Research of the National Bureau of Standards, volume 71B (1967), páginas 241-245. [17]

[1970] J. Edmonds, Submodular functions, matroids and certain polyhedra, em Combinatorial Structures and their Applications (Proceedings of the Calgary International Conference on Combinatorial Structures and their Applications, University of Calgary, June 1969), editado por R. K. Guy, H. Hanani, N. Sauer e J. Schönheim, Gordon and Breach, 1970, páginas 69-87. [116]

[1977] J. Edmonds E F. R. Giles, A min-max relation for submodular functions on graphs, em Studies in Integer Programming (Proceedings of the Workshop on Integer Programming, University of Bonn, September 8-12, 1975), editado por P. L. Hammer, E. L. Johnson, B. H. Korte e G. L. Nemhauser, volume 1 de Annals of Discrete Mathematics, North-Holland, 1977, páginas 185-204. [64, 65, 66, 92, 95, 131]

[1982] J. Edmonds, L. Lovász E W. R. Pulleyblank, Brick decompositions and the matching rank of graphs, Combinatorica: an International Journal on Combinatorics and the Theory of Computing, volume 2 (1982), páginas 247-274. [132]

[2006] F. Eisenbrand e G. Shmonin, Carathéodory bounds for integer cones, Operations Research Letters, volume 34 (2006), páginas 564-568. [132]

[1894] G. Farkas, A Fourier-féle mechanikai elv alkalmazásai, Mathematikai és Természettudományi Értesitö, volume 12 (1894), páginas 457-472. [25] 
[1999] R. T. Firla E G. M. ZIEgLeR, Hilbert bases, unimodular triangulations and binary covers of rational polyhedral cones, Discrete and Computational Geometry, volume 21 (1999), páginas 205-216. [132]

[1980] A. Frank, On the orientation of graphs, Journal of Combinatorial Theory (Series B), volume 28 (1980), páginas 251-261. [93]

[1984] A. Frank E É. Tardos, Matroids from crossing families, em Finite and Infinite Sets (Proceedings of the Sixth Hungarian Combinatorial Colloquium, Eger, July 6-11, 1981), editado por A. Hajnal, L. Lovász e V. T. Sós, volume 37 de Colloquia Mathematica Societatis Janos Bolyai, North-Holland, 1984, páginas 295-304. [93]

[1974] D. R. Fulkerson, Packing rooted directed cuts in a weighted directed graph, Mathematical Programming, volume 6 (1974), páginas 1-13. [95]

[1979] F. R. Giles E W. R. Pulleyblank, Total dual integrality and integer polyhedra, Linear Algebra and its Applications, volume 25 (1979), páginas 191-196. [62, 65, 66]

[1993] L. Goddyn, Cones, lattices and Hilbert bases of circuits and perfect matchings, em Graph Structure Theory (Proceedings of a Joint Summer Research Conference on Graph Minors held June 22 to July 5, 1991, at the University of Washington, Seattle), editado por N. Robertson e P. D. Seymour, volume 147 de Contemporary Mathematics, American Mathematical Society, 1993, páginas 419-439. [132]

[1873] P. GoRdan, Ueber die Auflösung linearer Gleichungen mit reellen Coefficienten, Mathematische Annalen, volume 6 (1873), páginas 23-28. [56]

[1981] M. Grötschel, L. LovÁsz E A. SchriJVer, The ellipsoid method and its consequences in combinatorial optimization, Combinatorica: an International Journal on Combinatorics and the Theory of Computing, volume 1 (1981), páginas 169-197. [99]

[1993] M. Grötschel, L. Lovász E A. SchriJVer, Geometric Algorithms and Combinatorial Optimization (Second Corrected Edition), volume 2 de Algorithms and Combinatorics, Springer, 1993. [116, 131]

[1984] N. KarmarkaR, A new polynomial-time algorithm for linear programming, em Proceedings of the Sixteenth Annual ACM Symposium on Theory of Computing (Washington, 1984), The Association for Computing Machinery, 1984, páginas 302-311. [34]

[1979] L. G. Khachiyan, A polynomial algorithm in linear programming, Soviet Mathematics Doklady, volume 20 (1979), páginas 191-194. [34, 131]

[2002] O. LEE E Y. WAKABAYASHI, On the circuit cover problem for mixed graphs, Combinatorics, Probability and Computing, volume 11 (2002), páginas 43-59. [132]

[1983] H. W. Lenstra JR., Integer programming with a fixed number of variables, Mathematics of Operations Research, volume 8 (1983), páginas 538-548. [68, 131]

[1982] A. K. Lenstra, H. W. Lenstra JR. E L. Lovász, Factoring polynomials with rational coefficients, Mathematische Annalen, volume 261 (1982), páginas 515-534. [131] 
[1991] J. LIU, Hilbert bases with the Carathéodory property, tese de doutorado, School of Operations Research and Industrial Engineering, New York, 1991. [132]

[1972] L. Lovász, Normal hypergraphs and the perfect graph conjecture, Discrete Mathematics, volume 2 (1972), páginas 253-267. [97]

[1983] L. LovásZ, Ear-decompositions of matching-covered graphs, Combinatorica: an International Journal on Combinatorics and the Theory of Computing, volume 3 (1983), páginas 105-117. [132]

[1987] L. LovÁsz, Matching structure and the matching lattice, Journal of Combinatorial Theory (Series B), volume 43 (1987), páginas 187-222. [132]

[1986] L. Lovász E M. D. Plummer, Matching theory, volume 29 de Annals of Discrete Mathematics, North-Holland, 1986. [132]

[1978] C. L. Lucchesi E D. H. Younger, A minimax theorem for directed graphs, Journal of the London Mathematical Society (Second Series), volume 17 (1978), páginas 369-374. [131]

[1896] H. Minkowski, Geometrie der Zahlen (Erste Lieferung), Teubner, 1896. [25]

[1994] U. S. R. MurTy, The Matching Lattice and Related Topics (Preliminary Report), relatório técnico, University of Waterloo, 1994. [114, 132]

[1982] D. J. NADDEF, Rank of maximum matchings in a graph, Mathematical Programming, volume 22 (1982), páginas 52-70. [132]

[1982] D. J. Naddef E W. R. Pulleyblank, Ear decompositions of elementary graphs and GF(2)-rank of perfect matchings, em Bonn Workshop on Combinatorial Optimization (August 28-30, 1980), editado por M. Grötschel, A. Bachem e B. H. Korte, volume 16 de Annals of Discrete Mathematics, North-Holland, 1982, páginas 241-260. [132]

[1961] C. St. J. A. Nash-Williams, Edge-disjoint spanning trees of finite graphs, Journal of the London Mathematical Society (Second Series), volume 36 (1961), páginas 445-450. [122]

[1969] C. St. J. A. Nash-Williams, Well-balanced orientations of finite graphs and unobtrusive odd-vertex-pairings, em Recent Progress in Combinatorics (Proceedings of the Third Waterloo Conference on Combinatorics, Waterloo, May 20-31, 1968), editado por W. T. Tutte, Academic Press, 1969, páginas 133-149. [93]

[1994] C. H. Papadimitriou, Computational Complexity, Addison-Wesley, 1994. [7]

[2003] J. C. DE PINA JR. E J. A. R. SoARes, Improved bound for the Carathéodory rank of the bases of a matroid, Journal of Combinatorial Theory (Series B), volume 88 (2003), páginas 323-327. [130]

[1981] A. Schrijver, On total dual integrality, Linear Algebra and its Applications, volume 38 (1981), páginas 27-32. [65, 67]

[1984] A. SchriJVer, Proving total dual integrality with cross-free families: a general framework, Mathematical Programming, volume 29 (1984), páginas 15-27. [131] 
[1986] A. SchriJver, Theory of Linear and Integer Programming, Wiley-Interscience Series in Discrete Mathematics and Optimization, John Wiley \& Sons, 1986. [25, 51, 58, 131]

[2003] A. SchriJver, Combinatorial Optimization: Polyhedra and Efficiency, volume 24 de Algorithms and Combinatorics, Springer, 2003. [131]

[1990] A. SEBŌ, Hilbert bases, Carathéodory's theorem and combinatorial optimization, em Integer Programming and Combinatorial Optimization (Proceedings of the 1st Integer Programming and Combinatorial Optimization Conference, Waterloo, May 28-30, 1990), editado por R. Kannan e W. Pulleyblank, University of Waterloo Press, 1990, páginas 431-455. [75, 86, 97, 132]

[1979] P. D. Seymour, Sums of circuits, em Graph Theory and Related Topics (Proceedings of the conference held in honour of Professor W. T. Tutte on the occasion of his sixtieth birthday, University of Waterloo, July 5-9, 1977), editado por J. A. Bondy e U. S. R. Murty, Academic Press, 1979, páginas 341-355. [103, 106, 112, 114, 115]

[1973] G. Szekeres, Polyhedral decompositions of cubic graphs, Bulletin of the Australian Mathematical Society, volume 8 (1973), páginas 367-387. [114]

[1961] W. T. TutTe, On the problem of decomposing a graph into $n$ connected factors, Journal of the London Mathematical Society (Second Series), volume 36 (1961), páginas 221-230. [122]

[1934] H. WeyL, Elementare Theorie der konvexen Polyeder, Commentarii Mathematici Helvetici, volume 7 (1934), páginas 290-306. [25] 



\section{Índice Remissivo}

árvore geradora, 8

$(u, v)$-caminho, 9

$c$-cobertura por cliques, 96

$c$-cobertura por cortes, 95

c-justo, 128

$r$-arborescência, 93

$r$-corte, 93

adaptação de vetor, 108

arco correspondente, 89

arestas coparalelas, 101

arestas correspondentes, 108

base de fecho linear, 22

base forte, 82

base livre de cruzamentos, 84

bloco, 120

cópia, 9

circuito, 8

clique, 96

cobertura dupla por circuitos, 114

cone, 33

conjunto estável, 96

contração de vértices, 107

correspondência biunívoca, 32

corte, 8

dimensão, 7

dual, 28

elemento forte, 82

eliminação gaussiana, 14

equação de dualidade, 28

equação linear, 11

expansão de vetor, 108

extensão de vetor, 105

face, 31 faceta, 32

família livre de cruzamentos, 92

fecho convexo, 8

fecho linear, 21

floresta, 8

floresta correspondente, 123

folgas complementares, 29

fonte, 123

forma $D_{r, k}, 14$

forma $T_{k}, 13$

grafo $k$-conexo, 89

grafo auxiliar, 123

grafo dirigido $k$-conexo, 89

grafo perfeito, 97

hiperplano suporte, 64

identificação, 108

igualdade implícita, 31

lema de Farkas, 27

linha ativa, 31

operações elementares sobre colunas, 7

operações elementares sobre linhas, 7

orientação, 89

pivoteamento, 73

poliedro, 27

poliedro inteiro, 63

pontudo, 35

posto coluna completo, 7

posto de Carathéodory, 88

posto linha completo, 7

programa inteiro, 63

programa linear, 27

programa linear inteiro, 63

resolver equação linear, 11 
resolver programa linear, 28

resolver sistema de desigualdades lineares, 27

resolver sistema linear, 12

restrição ativa, 31

restrição de sistema de desigualdades, 27

restrição de vetor, 105

restrição livre de igualdade, 31

simples, 8

sistema de desigualdades lineares, 27

sistema de equações lineares, 12

sistema linear, 12

sistema totalmente dual integral, 65

sistema totalmente dual integral minimal, 67

solução de equação linear, 11

solução de sistema de desigualdades lineares, 27

solução de sistema linear, 12

sorvedouro, 123

subsistema de igualdades implícitas, 31

subsistema livre de igualdades, 31

tamanho, 8

teorema das desigualdades lineares, 25

teorema da dualidade, 28

teorema da orientação, 93

teorema de Carathéodory, 35

teorema de Tutte e Nash-Williams, 126

teorema fundamental da álgebra linear, 16

tipo, 9

totalmente integral, 61

vértices de poliedro, 33

valor ótimo, 28

vetor admissível, 107

vetor de transferência, 119 